\title{
Unveiling the sensory connections between the bladder and the brain that involve the periaqueductal gray matter
}

Citation for published version (APA):

Zare, A. (2018). Unveiling the sensory connections between the bladder and the brain that involve the periaqueductal gray matter. [Doctoral Thesis, Maastricht University]. Maastricht University. https://doi.org/10.26481/dis.20180619az

Document status and date:

Published: 01/01/2018

DOI:

10.26481/dis.20180619az

Document Version:

Publisher's PDF, also known as Version of record

Please check the document version of this publication:

- A submitted manuscript is the version of the article upon submission and before peer-review. There can be important differences between the submitted version and the official published version of record.

People interested in the research are advised to contact the author for the final version of the publication, or visit the DOI to the publisher's website.

- The final author version and the galley proof are versions of the publication after peer review.

- The final published version features the final layout of the paper including the volume, issue and page numbers.

Link to publication

\footnotetext{
General rights rights.

- You may freely distribute the URL identifying the publication in the public portal. please follow below link for the End User Agreement:

www.umlib.nl/taverne-license

Take down policy

If you believe that this document breaches copyright please contact us at:

repository@maastrichtuniversity.nl

providing details and we will investigate your claim.
}

Copyright and moral rights for the publications made accessible in the public portal are retained by the authors and/or other copyright owners and it is a condition of accessing publications that users recognise and abide by the legal requirements associated with these

- Users may download and print one copy of any publication from the public portal for the purpose of private study or research.

- You may not further distribute the material or use it for any profit-making activity or commercial gain

If the publication is distributed under the terms of Article $25 \mathrm{fa}$ of the Dutch Copyright Act, indicated by the "Taverne" license above, 
Unveiling the sensory connections between the bladder and the brain that involve the periaqueductal gray matter

Aryo Zare

June 2018 


\section{About the cover:}

The two circles show cortical \& peripheral feedback loops related to the control of micturition, coordinated by specific PAG (The Hexagon) columns (dorsolateral \& ventrolateral).

Concept \& Design: Aryo Zare

Copyright: Aryo Zare (스yo-Zare@Outlook.com)

ISBN: 9789462339811

Printed by: Gildeprint - www.gildeprint.nl May 2018

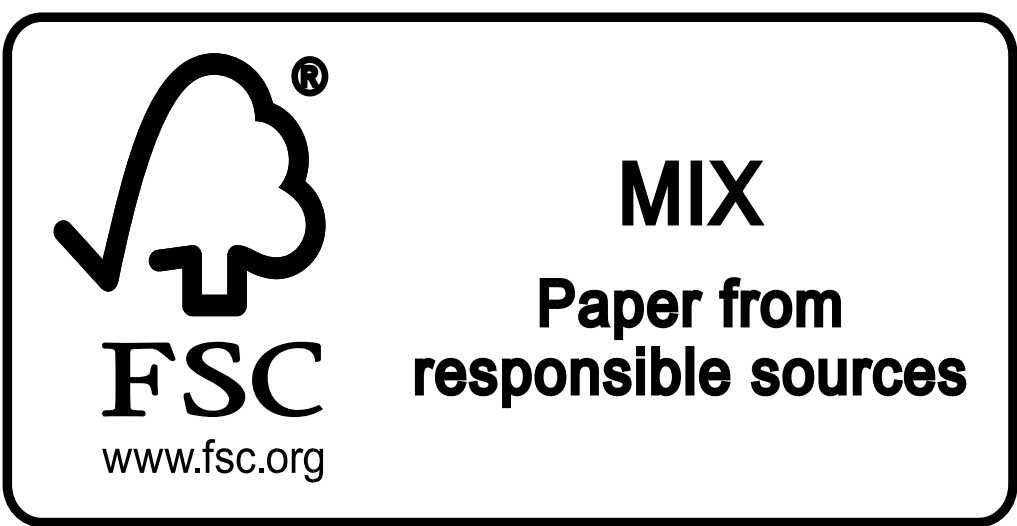




\section{Unveiling the sensory connections between the bladder and the brain that involve the periaqueductal gray matter}

To obtain the degree of Doctor at Maasstricht University

On the authority of the Rector Magnificus, Prof. Dr. Rianne M. Letschert

In accordance with the decision of the board of deans

To be defended in public on

June the $19^{\text {th }} 2018$, at $14: 00$

by

Aryo Zare 
Promoter: Prof. Dr. Gommert A. van Koeveringe

Copromoter: Dr. Ali Jahanshahi

Members of the assessment committee:

Prof. Dr. Harry W. M. Steinbusch (Chairman)

Prof. Dr. Jos Prickaerts

Prof. Dr. Bert Joosten

Prof. Dr. Matthias Oelke (Akademisches Lehrkrankenhaus WWU Munster)

Prof. Dr. Stefan De Wachter (University of Antwerpen, Belgium) 


\section{Contents}

Chapter 1

Chapter 2

Chapter 3

Chapter 4

Chapter 5

Chapter 6

Chapter 7

Chapter 8

Chapter 9

Valorization

Acknowledgement

Curriculum Vitae

Publications
General Introduction

The Role of the Periaqueductal Gray Matter in Lower

Urinary Tract Function. (Review article)

A novel implantation technique for neuromodulation of the 49 peripheral nervous system in rats.

Neuronal activation in the periaqueductal gray matter upon $\quad 59$ electrical stimulation of the bladder.

Glutamatergic cells in the periaqueductal gray matter 95 mediate sensory inputs following bladder stimulation in freely moving rats

Electrophysiological characteristics of neurons in the 107 ventrolateral periaqueductal gray and their correlation with bladder sensory signaling

Local Regulatory Changes in Isolated Bladders of Alzheimer 125 Mice

General Discussion

Summary 
Chapter 1

General introduction 


\section{General Introduction}

The prevalence of incontinence increases with age, affecting about $30 \%$ of elderly women and $15 \%$ of elderly men [1]. It has peak age of prevalence at 6th decade of life [2]. Some gender difference in prevalence exists, for example urge incontinence constitutes a larger portion of total urinary incontinence in men than in women. On the other hand mixed incontinence is much more common in women. In year 2000 urinary incontinence in women was the primary cause for over a million office visits in the United States [3]. In the same year, the total economic burden of urinary incontinence in the United States was estimated to be about $\$ 20$ billion [4]. As life expectancy increases and as the proportion of aging population in many societies expand, these numbers also tend to increase. This demands more attention to its prevention and management.

Risk factors for urinary incontinence include obesity, parity and mode of delivery in women, smoking, diabetes, vaginal atrophy, hormone replacement therapy and genitourinary surgery [5-15]. Stress incontinence is associated with participation in highimpact activities including running[16, 17]. Recurrent urinary tract infections, and childhood enuresis are additional risk factors for urgency incontinence [18]. Importantly urinary incontinence is common in patients with dementia, with the prevalence ranging from 10 to 38 percent[19]. Urinary incontinence does not increase mortality[20] but it decreases quality of life dramatically. It may also cause complications such as perineal infections secondary to candidiasis or cellulitis and aggravate decubitus ulcers[21].

Incontinence symptoms in general can be categorized into storage symptoms, voiding symptoms and post-micturition symptoms (detailed below). We here emphasize on the voiding symptoms which fall into two main categories; detrusor underactivity (underactive bladder, UAB) or bladder outlet obstruction. Of these two, the UAB is of particular interest to us as it involves some central etiologies behind it.

\section{Physiology of micturition}

As shown in Figure 1, bladder and urethral sphincters are in control of autonomic and somatic nervous systems[22, 23]. The spinal parasympathetic nerves emerge from sacral segments S2-3-4, on the other hand the sympathetic nerves to the bladder emerge from terminal thoracic and initial lumbar segments. They will then synapse at an intermediary neuron before finally reaching the organ. The synaptic stations defer in sympathetic and the parasympathetic nerves. The inferior hypogastric plexus forms a meshwork to distribute the final terminals of these neurons evenly throughout various parts of the organ. After emerging from the cord the sympathetic nerves pass the paravertebral sympathetic chain without synapsing. This chain provides a station for the nerve to move 


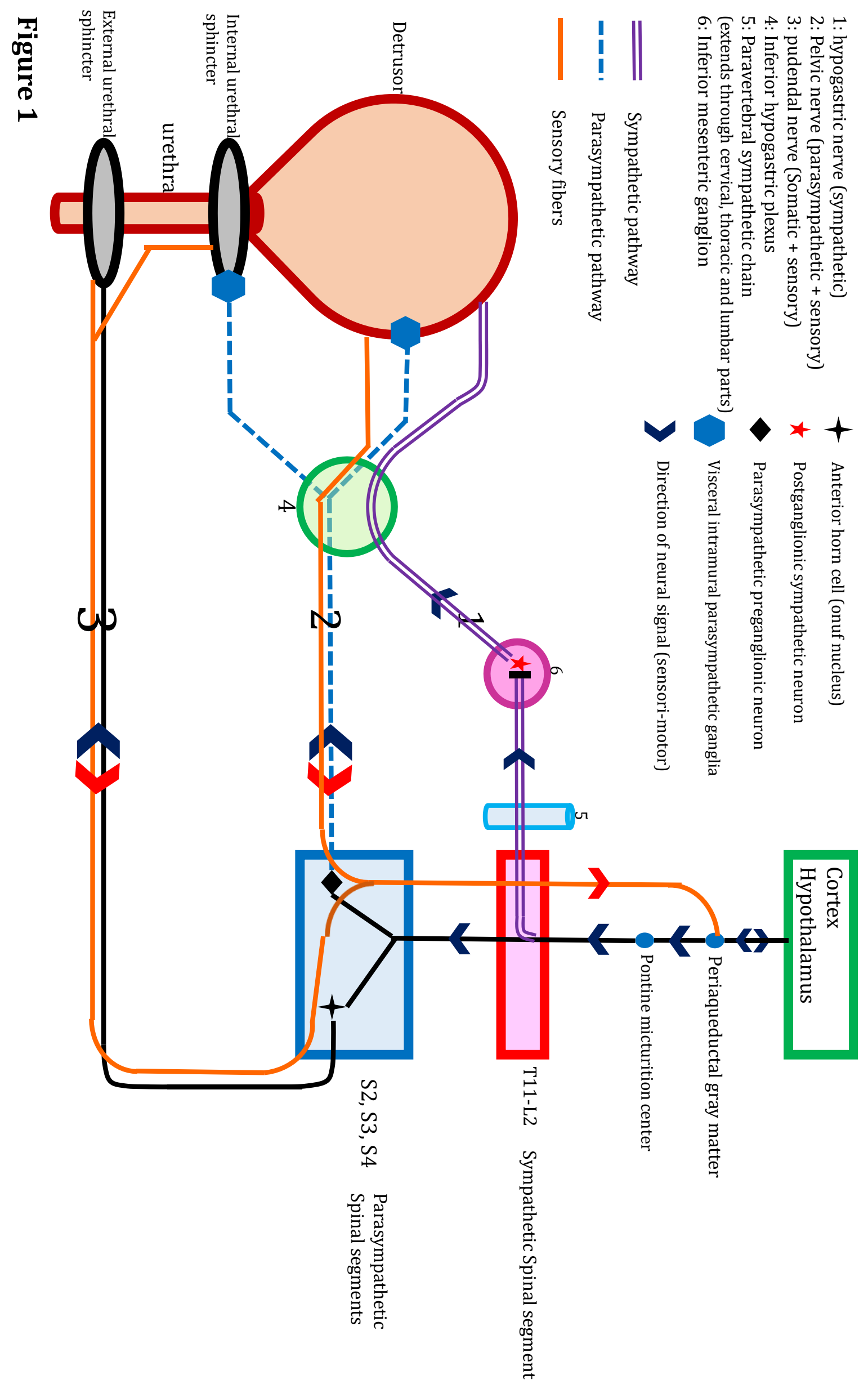


Figure 1. Neuroanatomy of the central and peripheral micturition control pathways.

to higher or lower nearby segments. In fact ganglionic cells of this chain provide a synaptic station for neurons reaching integumentary system or for those sympathetic nerves emerging from higher thoracic or cervical segments and will then reach thoracic visceral or cranial structures. The internal and external urethral sphincters provide autonomic and somatic (voluntary) control of micturition respectively. The somatic control is provided by the pudendal nerve which emerges form the anterior horn cells of the sacral segments, more precisely the Onuf's nucleus. The whole system is directly under the control of two critical brainstem locations namely the pontine micturition center and the periaqueductal gray matter of midbrain. They in turn interact with cortical regions to moderate the micturition behaviour after decision making.

The physiology of the bladder contraction depends on both detrusor muscle characteristics and possible locoregional chemical mediators affecting it, as well as nervous control by spinal and brain centers. The sum of this neuromuscular interaction defines the final contractile response of bladder. Pathological conditions may affect either the muscular component or nervous centers at various levels. Among spinal and cranial centers engaged in physiology of bladder contraction, the periaqueductal gray matter (PAG) has a cardinal role in coordination of storage and evacuation reflexes in the context of visceral and environmental factors.

\section{Pathophysiology}

Either motor or sensory dysfunctions may lead to functional bladder disorders. The sensory aspect of bladder dysfunction is a subject not much elaborated in the literature. Aside from motor control, pelvic and pudendal nerves also convey sensations of bladder fullness and sensory signals from the bladder neck respectively, whereas hypogastric nerve conveys both types of sensations to spinal centers[22]. The type of fibers also differ in these two afferents, the bladder filling information being conveyed by myelinated fibers and noxious stimuli conveyed by unmyelinated fibers. These signals finally reach S2-S4 or T11-L2 spinal segments. Non-neuronal cells within urothelium also contribute to sensory signal referral by interaction with suburothelia nerves. They have receptors for particular chemical mediators and can release various types of substances like ATP, acetylcholine and NO to indirectly affect vasomotor control of the bladder and its ultimate contractile responses.

Pathologic conditions may affect bladder sensation. Neuropathies are among the most common reasons of decreased bladder sensation, by which either myelinated or unmyelinated fibers may be affected[24]. One popular example of such diseases is diabetic neuropathy, which in parallel also affects skin sensation. Myelopathies like multiple sclerosis and familial spastic paraparesis affect the spinal afferent pathways and 
are the second most common cause of sensory bladder dysfunction. Similarly lumbar spondylosis may also lead to bladder sensory dysfunction. Bladder sensory dysfunction ultimately leads to less frequent voiding, bladder overdistension injury, and overflow incontinence. Unfortunately there is no sophisticated therapeutic option for bladder sensory dysfunction. Trials of imidapril which is an angiotensin-converting enzyme inhibitor have shown equivocal results.

On the other hand increased bladder sensation is also troublesome. Myelopathies are again a common cause of increased bladder sensation, spanning spinocerebellar ataxia type 6, SLE and Sjogren's syndrome among other etiologies. Neuropathies as occur in post-pelvic organ surgery, lumbar spondylosis, and diabetic neuropathy are the second most common cause of increased bladder sensation. This increased sensation is due to incomplete lesions affecting spinal afferent pathways, which will subsequently make spontaneously firing units in the spinal cord. This may be a result of ephaptic transmission of signals by adjacent irritated nerve fibers. Another possible reason for increased bladder sensation is cerebral pathologies like Parkinson's disease (PD). The PAG and the basal ganglia are among the structures involved in sensory gating which may be affected by various pathologies. Interestingly deep brain stimulation (DBS) of the subthalamic nucleus in PD ameliorated some sensory bladder dysfunctions in PD.

The control over continence depends on structural, neurological and physiological factors arranging the lower urinary tract. This control system continues to develop in infancy and matures in early childhood. Though collectively called urinary incontinence, various subtypes of conditions exist that correspond to this symptom:

Lower urinary tract symptoms (LUTS) is a nonspecific term referring to any combination of storage, voiding or postmicturition symptoms[25, 26]:

- Storage symptoms: are experienced during the bladder filling

○ Urgency - a complaint of sudden and compelling desire to void

- Urgency incontinence is leakage following urgency.

- Stress incontinence is leakage associated with exertion or coughing.

- Mixed incontinence is a combination of urgency and stress incontinence.

- Overactive bladder is a syndrome consisting of urgency, frequency, and nocturia, with or without incontinence.

- Overflow incontinence (false incontinence) is a term used when a flaccid bladder reaches its maximum volume threshold and ends up with leakage.

- Daytime frequency - Too often voiding by day

- Nocturia - At least once waking up at night for voiding.

- Coital incontinence - occurs during sexual activity.

- Voiding symptoms: are experienced at the time of voiding

- Slow stream - A relatively reduced urinary flow rate.

- Intermittent stream or intermittency - interruption of urine flow during micturition. 
- Hesitancy - delay in the onset of voiding

- Straining to void - Increasing intra-abdominal pressure by diaphragmatic or abdominal muscular effort.

○ splitting or spraying: non-smooth urine stream while voiding.

- Terminal dribble - Prolongation of the final phase of voiding.

○ Dysuria - discomfort at the time of voiding.

- Post-micturition symptoms

- A sensation of residual urine within the bladder after voiding.

- Postvoid dribbling describes dribbling of residual urine in the urethra after the bladder evacuation.

Voiding dysfunction, sometimes stated as incomplete bladder emptying is one of the main manifestations of sensory bladder pathologies. This consists of abnormally slow and/or incomplete micturition. Dysfunctional voiding is a similar entity that ICS defines as "intermittent and/or fluctuating flow rate due to involuntary intermittent contractions of the peri-urethral striated muscle during voiding in neurologically normal individuals"

Voiding dysfunction has various etiologies as depicted below[27] :

- Detrusor underactivity (underactive bladder, UAB)

○ Neuropathic

- decentralization

- Infection

- Diabetes

- PD

- Multiple system atrophy

- Damage to lower motor neurons

○ Myogenic

- Overdistention

- Aging

- Pharmacologic

- Anticholinergics

- Alpha-agonists

- Narcotics

- Anesthetics

- Bladder outlet obstruction

- Fixed anatomic obstruction

- Men

- Stricture

- Prostate enlargement

- Women

- Pelvic organ prolapse

- Extrinsic compression

- Caruncle 


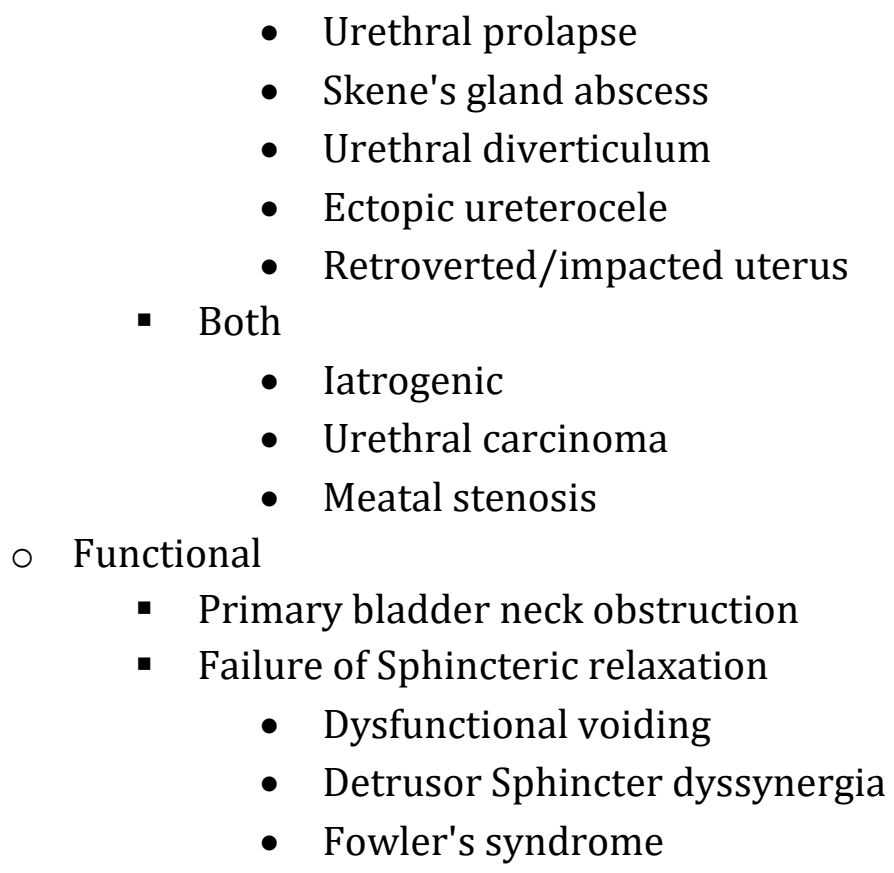

$\mathrm{UAB}$ as a common form of voiding dysfunction is defined as reduced strength and/or duration of contraction leading to prolonged bladder emptying and/or a failure to completely empty the bladder. UAB can be observed in many neurologic and myogenic conditions [28]. The most common of which is diabetic cystopathy. The prevalence of UAB spans $9-28 \%$ of men $<50$ year of age increasing to about $48 \%$ in men $>70$ year. In the elderly women, prevalence ranges from $12 \%$ to $45 \%$ [29].

\section{Table 1. Some common treatment modalities for urinary incontinence}

- Pharmacotherapy

- Anticholinergics (eg. Oxybutinin)

- Sympatholytics (eg. Prazosin)

○ Hormonal antimetabolites (eg. Finasteride)

- Surgery

- Augmentation cystoplasty

○ Urinary diversion

o Women :

- Midurethral sling

- Pubovaginal sling

- Urethral/colposuspension

- Anti-incontinence pessary (women)

- Functional electrical stimulation (peripheral neuromodulation)

- Pelvic floor muscle training

- Behavioral therapy

- Fluid management 


\section{Treatment of urinary incontinence}

Table 1. summarizes common treatment modalities for urinary incontinence. As seen almost none of them focuses on possible central etiologies, including those originating in brainstem areas controlling the micturition reflex i.e. pontine micturition center and the periaqueductal gray matter. Multiple sclerosis (MS), PD, cerebral stroke, multiple system atrophy and neurodegenerative disorders are some brain disorders which may cause incontinence and have the PAG as part of their pathologically affected regions. This highlights the lack of sufficient diagnostic and therapeutic investigation on central structures.

\section{Periaqueductal gray matter, the headquarter of bladder control}

Among central nervous system (CNS) modulators of bladder function we redirected our attention to PAG. The PAG is the central gray matter of the midbrain. Due to its axial location it is involved in various important functions including behavioural[30], visceral[31] and autonomic[32, 33] control. The role of PAG in control of the bladder function encompasses both spinal and cortical connections. The PAG has functionally[34] and histologically[35] separate columns. These columns can be divided into two functionally separate groups having opposite autonomic functions, the ventrolateral column having parasympathetic function and the lateral and dorsolateral columns which have sympathetic functions[36, 37]. All of the PAG columns have reciprocal ipsilateral and contralateral connections with each other as well as internal connections within each column[38]. We reviewed and digested the current knowledge about PAG and bladder function in chapter 2 .

Neuromodulation of peripheral nervous system is a growing modality in various disciplines. Sacral neuromodulation in particular has been used in humans for various functional pelvic pathologies including urinary incontinence[39], fecal incontinence [40] and female pelvic floor dysfunction [41]. Since the PAG integrates the central and peripheral nervous system, and voluntary and involuntary control of micturition, it is a pivotal center for control of voiding and continence. This makes it a favorite candidate for surgical neuromodulation. Indeed attempts were made to modulate the micturition through DBS of the PAG. DBS of the ventrolateral column of the PAG (vlPAG) suppressed voiding in rats and humans[42]. However the brainstem mainly due to its many different tracts and connections being concentrated in a small area is a risky region for surgical interventions.

For understanding the mechanism of such therapeutic interventions, reliable animal models are required. Rodents have a natural tendency to damage devices attached to them. Alternative approaches like using a body harness with an attached backpack have their own disadvantages. We tried to tackle this problem by a different implantation technique, exploiting the rodents' loose skin jacket and subcutaneous tissue to guide the wires. 
The first step for performing a successful neuromodulation is determination of exact location for stimulation. We designed an experimental setup to differentiate the response of various PAG columns to electrical bladder stimulation. The activated neurons were recognized by determination of the level of their cFos expression. Aside from exploration of the anatomical pathways between bladder and the PAG and their stimulatory or inhibitory function, it is of interest to define particular types of neurons involved in this communication. The cell type characterization not only helps to understand the pathophysiology of particular diseases but also has the opportunity to identify new identify pharmacotherapeutic modalities. Various studies defined the vlPAG as the column responsible for control of bladder function[43-46]. We aimed to characterize the neurotransmitter phenotype of micturition related cells in the vlPAG. We tested major stimulatory and inhibitory neurotransmitters, which are known to be expressed by PAG neurons.

Various studies have been performed to neurophysiologicaly characterize the PAG neurons involved in micturition. These studies consist of either electrophysiological recordings of single units or local field potentials or electrical stimulation of PAG columns. Single unit recordings in the vlPAG shows different patterns of neuronal firing rate during micturition[47], which may correspond to specific functions of these clusters of neurons. Studies also showed that detrusor contraction along with a specific vlPAG firing pattern varies according to different EEG activity phases in rats under anesthesia[48] which may shed a new light on pathophysiology of nocturnal enuresis. On the other hand electrical PAG stimulation results in peripheral responses. Electrical stimulation of the vlPAG may either cause a contraction or inhibition of the bladder[49]. In this thesis we started to define electrophysiological characteristics of vlPAG neurons after electrical bladder stimulation.

The PAG can be affected in various neuropathological conditions affecting micturition. The potential role of the PAG in the pathogenesis of micturition problems merits consideration. Our main question is to find out if the PAG would be a feasible target for either electrical but also possible pharmacological or otherwise targeted neuromodulation and if so which exact location at this structure may be stimulated. We hypothesize that targeting PAG either by neuromodulation or pharmacotherapeutical targeting of its specific receptors, may modulate specific ascending pathways reaching the PAG and may ultimately ameliorate patient symptoms.

As not enough attention was devoted to the PAG and its role in functional bladder disorders so far, in chapter 2 we aimed for a systematic review of the literature to clarify its physiological role in bladder function, outcomes of its pathological states in bladder physiology and possible diagnostic tools which may show its pathology and would be capable of being utilized in diagnostic algorithms. Our aim was to find out in what extent the research has been performed on this topic and which direction would be the best to 
continue this. This encompasses normal and pathologic states, anatomical and physiological perspectives, as well as diagnostic and therapeutic implications.

As we considered using neuromodulation in some functional bladder disorders, in chapter 3 we figured out a proper technique to be used in laboratory rats for this purpose. Our intention was to implement a method on freely moving rats which would both be comfortable for the animal and easy to perform by the experimentalist. Our objective was to mount a plugging construction on an area over the rat body so that it would not be manipulated and damaged by the animal in long term. Our approach was taking advantage of the rat's loose jacket to traverse the wires toward the cranial surface.

Previous research had defined the PAG to be an afferent station of bladder sensory signals. The PAG is composed of different columns consisting of 3 pairs of ventrolateral, lateral and dorsolateral columns and on dorsomedial column. Since different PAG columns are involved in various functions, in chapter 4 we aimed for defining the exact column which receives the afferent sensory signals. We hypothesized that after stimulating the bladder a particular column would show more activation and would be involved in processing of sensory bladder signals. To this end we used electrical bladder stimulation and c-Fos labeling of activated cells to find out the involved column.

In chapter 5 we aimed for defining various cell types in PAG which may receive these signals from the bladder. Our hypothesis was activation of a certain type of neurotransmitter of either stimulatory or inhibitory type following bladder stimulation. We used co-labelling of cell activation marker (c-Fos) with specific neurotransmitters to explore cell types.

Electrophysiological waveform and firing pattern, as another physiological characteristic of neurons, can be sought to both categorize neurons and evaluate their response to particular stimuli. Chapter 6 describes the electrophysiological characteristics of PAG neurons. We wanted to find out how the PAG neurons' firing pattern would change following electrical bladder stimulation. This would have given us an overall idea of excitation or inhibition of these neurons. This would subsequently shed light on their specific function and their possible role among other central micturition related areas as pontine micturition center or cortical regions. To accomplish this we electrically stimulated the bladder with previously defined parameters to stimulate sensory signals and simultaneously recorded single units within the PAG and observed their reaction.

As PAG can be affected in neurodegenerative diseases including Alzheimer disease (AD), it can be a possible diagnostic or therapeutic target for continence problems in this patient group. Before focusing on targeting this structure for diagnostic or therapeutic purposes we must ensure that central etiologies have a significant role in pathogenesis of this disease. The initial step before considering a therapeutic approach toward the PAG for any disease is to weigh the level of involvement of various factors leading to patient symptoms. $\mathrm{AD}$ is one of the most prevalent neurodegenerative diseases. AD patients suffer from bladder overactivity[50]. In chapter 7 we aimed to determine the relative contribution of the central and the peripheral nervous system to bladder pathophysiology in this disease. This chapter tests the hypothesis whether the bladder would still function abnormally in AD subjects after excluding CNS interference. This is a 
comprehensive step which must be taken before definitive PAG research in AD. Using transgenic animals we aimed to define the structural or physiological changes in bladders of $\mathrm{AD}$ mice. We assessed the organ specific pathophysiology of an AD model organism, using an organ bath setting. This ex-vivo experiment helps to exclude any possible CNS interference contributing to bladder dysfunction. Decision for considering the PAG as a neuromodulation or alternative therapy target for any particular disease is only feasible after proving the significance of the central pathogenetic role or therapeutic potential of PAG in that specific disease.

In chapter 8 we will discuss the whole topic in conclusion.

\section{References}

1. Porter, R.S., The Merck Manual of Diagnosis and Therapy. 2011: Wiley.

2. Nitti, V.W., The prevalence of urinary incontinence. Rev Urol, 2001. 3 Suppl 1: p. S2-6.

3. Litwin, M.S., et al., Urologic diseases in America Project: analytical methods and principal findings. J Urol, 2005. 173(3): p. 933-7.

4. Hu, T.W., et al., Costs of urinary incontinence and overactive bladder in the United States: a comparative study. Urology, 2004. 63(3): p. 461-5.

5. Subak, L.L., H.E. Richter, and S. Hunskaar, Obesity and urinary incontinence: epidemiology and clinical research update. J Urol, 2009. 182(6 Suppl): p. S2-7.

6. Subak, L.L., et al., Weight loss to treat urinary incontinence in overweight and obese women. N Engl J Med, 2009. 360(5): p. 481-90.

7. Elia, G., J. Bergman, and T.D. Dye, Familial incidence of urinary incontinence. Am J Obstet Gynecol, 2002. 187(1): p. 53-5.

8. Hannestad, Y.S., et al., Familial risk of urinary incontinence in women: population based cross sectional study. BMJ, 2004. 329(7471): p. 889-91.

9. Rortveit, G., et al., Age- and type-dependent effects of parity on urinary incontinence: the Norwegian EPINCONT study. Obstet Gynecol, 2001. 98(6): p. 1004-10.

10. Lukacz, E.S., et al., Parity, mode of delivery, and pelvic floor disorders. Obstet Gynecol, 2006. 107(6): p. 1253-60.

11. Dallosso, H.M., et al., The association of diet and other lifestyle factors with overactive bladder and stress incontinence: a longitudinal study in women. BJU Int, 2003. 92(1): p. 6977.

12. Tahtinen, R.M., et al., Smoking and bladder symptoms in women. Obstet Gynecol, 2011. 118(3): p. 643-8.

13. Melville, J.L., et al., Urinary incontinence in US women: a population-based study. Arch Intern Med, 2005. 165(5): p. 537-42.

14. Grodstein, F., et al., Association of age, race, and obstetric history with urinary symptoms among women in the Nurses' Health Study. Am J Obstet Gynecol, 2003. 189(2): p. 428-34.

15. Manson, J.E., et al., Menopausal hormone therapy and health outcomes during the intervention and extended poststopping phases of the Women's Health Initiative randomized trials. JAMA, 2013. 310(13): p. 1353-68.

16. Fozzatti, C., et al., Prevalence study of stress urinary incontinence in women who perform high-impact exercises. Int Urogynecol J, 2012. 23(12): p. 1687-91.

17. Goldstick, O. and N. Constantini, Urinary incontinence in physically active women and female athletes. Br J Sports Med, 2014. 48(4): p. 296-8. 
18. Keller, D.L., Idiopathic urgency urinary incontinence. N Engl J Med, 2010. 363(27): p. 2672; author reply 2672.

19. Drennan, V.M., et al., The prevalence of incontinence in people with cognitive impairment or dementia living at home: a systematic review. Neurourol Urodyn, 2013. 32(4): p. 314-24.

20. Herzog, A.R., et al., Urinary incontinence as a risk factor for mortality. J Am Geriatr Soc, 1994. 42(3): p. 264-8.

21. Brown, J.S., et al., Urinary incontinence: does it increase risk for falls and fractures? Study of Osteoporotic Fractures Research Group. J Am Geriatr Soc, 2000. 48(7): p. 721-5.

22. Fowler, C.J., D. Griffiths, and W.C. de Groat, The neural control of micturition. Nat Rev Neurosci, 2008. 9(6): p. 453-66.

23. Gray, H., et al., Gray's Anatomy: The Anatomical Basis of Clinical Practice. 2005: Elsevier Churchill Livingstone.

24. Tsunoyama, K., et al., Pathogenesis of reduced or increased bladder sensation. Neurourol Urodyn, 2011. 30(3): p. 339-43.

25. Kevin T McVary, M., FACSRajiv Saini, MD, Lower urinary tract symptoms in men, M. Michael P O'Leary, MPH, Editor. 2017, UpToDate (Accessed on 2018-1-20).

26. J Quentin Clemens, M., FACS, MSCI, Urinary incontinence in men, M. Michael P O'Leary, MPH, Howard Libman, MD, FACP, Editor. 2017, UpToDate (Accessed on 2018-1-22).

27. Nitti, V. and A. Khan, Retention and Bladder-Emptying Disorders, in Practical Functional Urology, J. Heesakkers, et al., Editors. 2016, Springer International Publishing: Cham. p. 353-370.

28. Miyazato, M., N. Yoshimura, and M.B. Chancellor, The other bladder syndrome: underactive bladder. Rev Urol, 2013. 15(1): p. 11-22.

29. Osman, N.I., et al., Detrusor underactivity and the underactive bladder: a new clinical entity? A review of current terminology, definitions, epidemiology, aetiology, and diagnosis. Eur Urol, 2014. 65(2): p. 389-98.

30. Mouton, L.J. and G. Holstege, The Periaqueductal Gray in the Cat Projects to Lamina-Viii and the Medial Part of Lamina-Vii Throughout the Length of the Spinal-Cord. Experimental Brain Research, 1994. 101(2): p. 253-264.

31. Budai, D., I. Harasawa, and H.L. Fields, Midbrain periaqueductal gray (PAG) inhibits nociceptive inputs to sacral dorsal horn nociceptive neurons through alpha(2)-adrenergic receptors. Journal of Neurophysiology, 1998. 80(5): p. 2244-2254.

32. Rathner, J.A. and S.F. Morrison, Rostral ventromedial periaqueductal gray: a source of inhibition of the sympathetic outflow to brown adipose tissue. Brain Res, 2006. 1077(1): p. 99-107.

33. Farkas, E., A.S. Jansen, and A.D. Loewy, Periaqueductal gray matter input to cardiac-related sympathetic premotor neurons. Brain Res, 1998. 792(2): p. 179-92.

34. Bandler, R. and M.T. Shipley, Columnar organization in the midbrain periaqueductal gray: modules for emotional expression? Trends Neurosci, 1994. 17(9): p. 379-89.

35. Parvizi, J., G.W. Van Hoesen, and A. Damasio, Selective pathological changes of the periaqueductal gray matter in Alzheimer's disease. Annals of Neurology, 2000. 48(3): p. 344-353.

36. Benarroch, E.E., Periaqueductal gray: an interface for behavioral control. Neurology, 2012. 78(3): p. 210-7.

37. Linnman, C., et al., Neuroimaging of the periaqueductal gray: state of the field. Neuroimage, 2012. 60(1): p. 505-22. 
38. Jansen, A.S.P., et al., Local connections between the columns of the periaqueductal gray matter: a case for intrinsic neuromodulation (vol 784, pg 329, 1998). Brain Research, 1998. 797(2): p. 368-368.

39. Sukhu, T., M.J. Kennelly, and R. Kurpad, Sacral neuromodulation in overactive bladder: a review and current perspectives. Res Rep Urol, 2016. 8: p. 193-199.

40. Rosen, A., et al., Effects of Sacral Neuromodulation on Urinary and Fecal Incontinence. Isr Med Assoc J, 2015. 17(6): p. 351-5.

41. Pettit, P.D., J.R. Thompson, and A.H. Chen, Sacral neuromodulation: new applications in the treatment offemale pelvic floor dysfunction. Curr Opin Obstet Gynecol, 2002. 14(5): p. 5215.

42. Green, A.L., et al., Switching Off Micturition Using Deep Brain Stimulation at Midbrain Sites. Annals of Neurology, 2012. 72(1): p. 144-147.

43. Mitsui, T., et al., Chemical bladder irritation provokes c-fos expression in the midbrain periaqueductal gray matter of the rat. Brain Res, 2003. 967(1-2): p. 81-8.

44. Taniguchi, N., et al., A study of micturition inducing sites in the periaqueductal gray of the mesencephalon. J Urol, 2002. 168(4 Pt 1): p. 1626-31.

45. Duong, M., J.W. Downie, and H.J. Du, Transmission of afferent information from urinary bladder, urethra and perineum to periaqueductal gray of cat. Brain Research, 1999. 819(12): p. 108-119.

46. Matsuura, S., G.V. Allen, and J.W. Downie, Volume-evoked micturition reflex is mediated by the ventrolateral periaqueductal gray in anesthetized rats. Am J Physiol, 1998. 275(6 Pt 2): p. R2049-55.

47. MATSUMOTO Kengo, S.T., YAMAMOTO Gen Characterization of micturition-related single neuron activities and their distribution in periaqueductal gray matter (PAG) in anesthetized rats. J Pharmacol Sci 2006. 100: p. 251.

48. Crook, J. and T. Lovick, Urodynamic function during sleep-like brain states in urethane anesthetized rats. Neuroscience, 2016. 313: p. 73-82.

49. Noto, H., et al., Excitatory and inhibitory influences on bladder activity elicited by electrical stimulation in the pontine micturition center in the rat. Brain Res, 1989. 492(1-2): p. 99115.

50. Lee, S.H., et al., Urinary incontinence in patients with Alzheimer's disease: Relationship between symptom status and urodynamic diagnoses. International Journal of Urology, 2014. 21(7): p. 683-687. 


\section{Chapter 2}

\section{The Role of the Periaqueductal Gray Matter in Lower Urinary}

Tract Function.

Aryo Zare, Ali Jahanshahi, Mohammad-Sajjad Rahnama'i, Sandra Schipper, Gommert A. van Koeveringe

“Molecular Neurobiology" DOI: 10.1007/s12035-018-1131-8 


\begin{abstract}
The periaqueductal gray matter (PAG), as one of the mostly preserved evolutionary components of the brain, is an axial structure modulating various important functions of the organism, including autonomic, behavioural, pain and micturition control. It has a critical role in urinary bladder physiology, with respect to storage and voiding of urine. The PAG has a columnar composition, and has extensive connections with its cranially and caudally located components of the central nervous system (CNS). The PAG serves as the control tower of the detrusor and sphincter contractions. It serves as a bridge between the evolutionary higher decision-making brain centers, and the lower centers responsible for reflexive micturition. Glutamatergic cells are the main operational neurons in the vlPAG, responsible for the reception and relay of the signals emerging from the bladder, to related brain centers. Functional imaging studies made it possible to clarify the activity of the PAG in voiding and filling phases of micturition, and its connections with various brain centers in living humans. The PAG may be affected in a wide spectrum of disorders, including multiple sclerosis(MS), migraine, stroke, Wernicke's encephalopathy and idiopathic normal pressure hydrocephalus, all of which may have voiding dysfunction or incontinence, in certain stages of the disease. This emphasizes the importance of this structure for the basic understanding of voiding and storage disorders and makes it a potential candidate for diagnostic and therapeutic interventions.
\end{abstract}

\title{
Keywords;
}

periaqueductal gray matter; bladder; incontinence; micturition; brain

\section{Introduction}

The PAG is the central gray matter of the midbrain, in continuance with the circumventricular organs, and is to a large extent analogous to the gray matter of the spinal cord. Due to its axial location, the PAG is involved in various important functions, including autonomic [1, 2], behavioural [3], pain [4] and micturition control. The role of the PAG in the control of the bladder function encompasses both downstream connections, as well as connections with the higher brain centers involved in decision making. Barrington was the first one to discover the function of the pontine micturition center (PMC)(Barrington's nucleus), and the role of the midbrain in the control of micturition, though he did not mention the PAG [5].

The PAG has been found to bear functionally separate columns [6], which are also different histopathologically in the human brain [7]. There are three pairs of columns, namely ventrolateral (vlPAG), lateral (lPAG) and dorsolateral (dlPAG), as well as a single dorsomedial column (dmPAG), in the PAG(Figure 1). These columns can be functionally 
divided into two groups, having opposite autonomic functions: the ventrolateral column, which has parasympathetic functions, and the lateral and dorsolateral columns which have sympathetic functions [8, 9]. Even considering the amount of expression of specific markers, or distant connections, there exist two distinct components: the dorsolateral pair of columns, and the remaining columns, including the ventrolateral, lateral and dorsomedial pairs[10]. dlPAG is functionally more closely related to the midbrain tectum, and the remaining columns may be considered more closely related to the periventricular structures [10]. All of the PAG columns have ipsilateral and contralateral reciprocal connections [11]. Internal connections also exist within each column [11]. Below we will discuss the role of the PAG in the control of micturition.

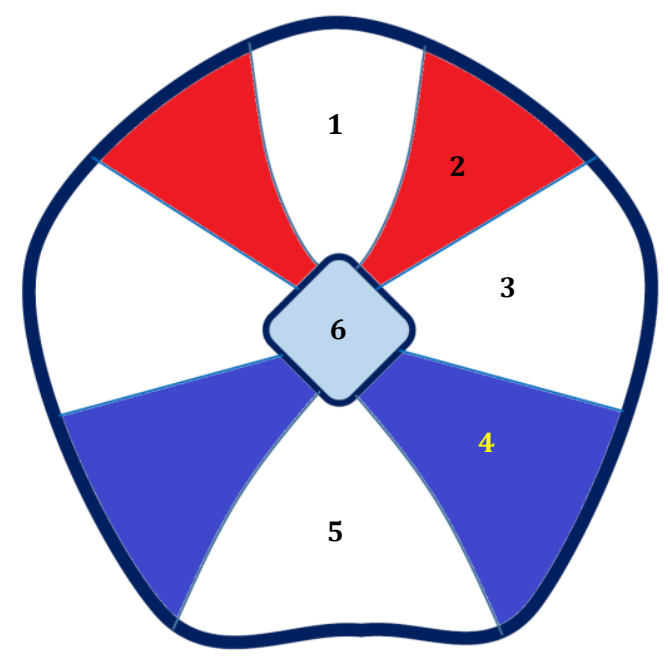

Figure 1. Schematic diagram of a coronal section through the rat PAG showing columnar segmentations. Two pairs of columns with major functional contribution in micturition, namely dorsolateral and ventrolateral, are highlighted in red and blue, respectively.

1. Dorsomedial column

2. Dorsolateral column (red)

3. Lateral column

4. Ventrolateral column (blue)

5. Area of dorsal raphea and some cranial nerve nuclei.

6. Sylvian aqueduct.

\section{Connections of the PAG}

The PAG has extensive connections with the cortex (prefrontal, cingulate and insular gyri), diencephalon (thalamus and medial preoptic area of hypothalamus(MPO)), brainstem(PMC) and the spinal cord (sacral segments)(figure 2)[12-18]. Caudal connections of the PAG have been investigated by tracing techniques, which are briefly mentioned in this section. The cranial connections with cortical regions have been discovered mainly by connectivity analysis in functional imaging studies, which will be described in the following sections. 
PMC, as another important micturition control center, has bilateral connections with vlPAG [16, 17, 19], as well as receiving afferents from dlPAG and the MPO [16, 20, 21](Figure 2). In parallel, MPO has direct connections with the PAG [22-24]. The MPO, rich in androgen and estrogen receptors, is integral to the limbic, or the so called emotional motor system, regulating sexual behavior. Since micturition is an important behavioural signal in animal territorial demarcation, and the scent of urine may additionally serve as a sexual message, the PAG may integrate the micturition and mating functions.

The dIPAG along with vlPAG receive afferents from spinal parasympathetic and dorsal commissural nuclei of the lumbosacral cord [25-27], which have ipsilateral dominance [25]. This pathway contributes to the awareness of the PAG to the state of the bladder fullness. Efferent PAG connections project indirectly via PMC to the spinal segments, and are distributed via somatic or autonomic nerves and ganglia to the detrusor muscle or sphincters [28].

\section{The vIPAG as the major PAG column with connections to the bladder}

The vlPAG has direct connections with the spinal segments [29]. It is also the main column receiving afferents from the lumbosacral cord, which have ipsilateral dominance [25], and originate from laminae V, VII, and VIII [29]. Spinal neurons throughout the cord, projected more to the IPAG and the VIPAG, rather than to the dIPAG or the AmPAG, and specially segments from the S1-S3, projected to the central portion of the IPAG and the vlPAG [30]. Indeed, only few neurons, mainly located in the lumbosacral segments, project to the dorsomedial and dorsolateral PAG columns [31]. The quantity of projections weighs toward the ventrolateral column, more than to the other columns, and there exist evidence showing the higher significance of the role of the vlPAG, regarding the control of bladder function.

Further evidence regarding the importance of vlPAG in micturition control are as follows. Most c-Fos reactivity is induced in the vlPAG after chemical irritation [32] or electrical stimulation (unpublished data) of the bladder. c-Fos is a transcription factor expressed after neuronal activation. Electrical stimulation of the pelvic nerves in the cat, evoked maximum field potentials in vlPAG [33]. Studies in cats confirm that it's the vlPAG, of which the electrical or chemical (DL-homocysteine) stimulation result in the contraction of the bladder [17]. Chemical stimulation of the vIPAG by D,L-homocysteic acid (DLH) in rats increases the frequency of micturition [34]. On the other hand, stereotaxic injection of the inhibitory mediator cobalt chloride into the caudal vlPAG reversibly attenuates bladder contractions and external urethral sphincter (EUS) electromyographic activity in rats [35]. Injection of other inhibitory or stimulatory agents, $\mathrm{CoCl} 2$ and L-glutamate, into the vlPAG lead to suppression or stimulation of voiding in rats, respectively [36]. Taking these studies into account, it is most likely that the vlPAG would be the primary station of the ascending PAG afferents, and it may secondarily relay these signals to the other PAG columns [11]. 


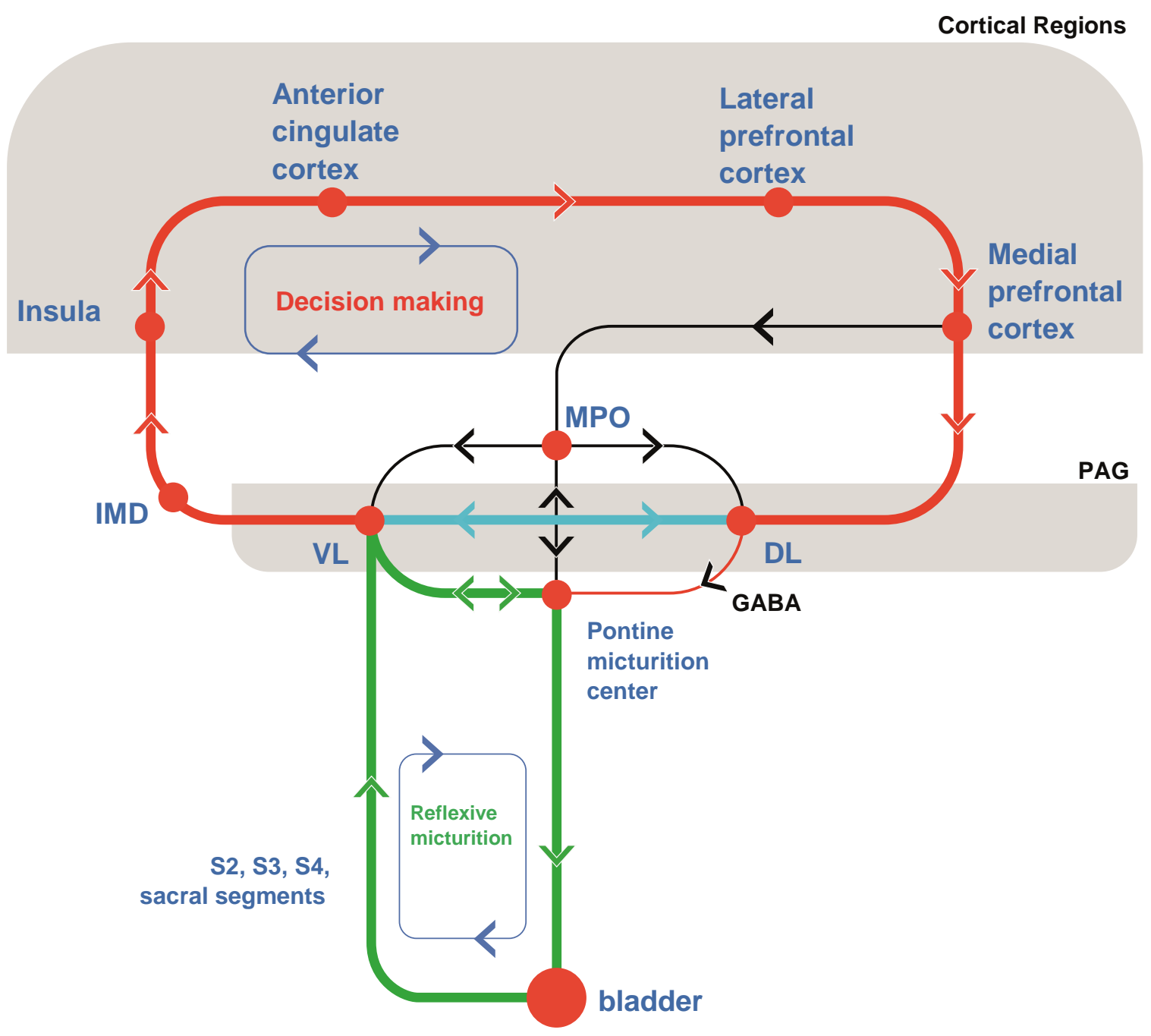

Figure 2. Major central pathways of micturition. The bladder distension signals, first reach directly to the ventrolateral column of the PAG[25,29]. This may trigger the PMC and induce the contraction of the bladder without any interference from the higher brain centers, which may be the underlying reason of infantile incontinence, or the often-reflexive micturition in rats. Though rats sometimes decide to demarcate their territory by the scent of urine. The thalamus does not receive direct signals from the bladder or the spinal cord. Instead, these sensory signals must first synapse in the PAG. The vlPAG projects to the intermediodorsal nucleus of the thalamus, and then the insula, in rat [137]. These signals finally reach the medial prefrontal cortex[13]. The projections from medial prefrontal areas predominantly reach the dorsolateral PAG columns[136]. It's noteworthy to mention that the existence of the prefrontal cortex in rodents is controversial. The involvement of the medial preoptic area $[96,105]$ may provide additional safe signaling for the start of voiding, or may even serve as a coordinating center to harmonize the voiding with mating or territorial demarcation behaviour[138]. There's a profound network of intercolumnar connections in the PAG[11] which provide continued processing of the incoming signals of the level of bladder fullness with the environmental state of the person and decide at the feasible opportunity to void. The brain pathway has been shown by various circuits [12-15] according to characteristics found in functional imaging, but specifc PAG columns could not be defined yet due to deficient spacial resolution of the imaging modalities. 
VL: ventrolateral column, DL : dorsolateral column, IMD : intermediodorsal nucleus of the rat thalamus [ analogous to mediodorsal nuclei of human Thalamus ], MPO : Medial preoptic area of hypothalamus

\section{PAG and the function of the bladder}

Functional studies regarding the control of the PAG over the bladder encompass electrical or chemical stimulation of the PAG, single unit recordings in the PAG, or indirect evaluation of the function by measuring the neuronal markers c-Fos or nerve growth factor (NGF). We can differentiate two major columns, the ventrolateral and the dorsolateral columns, for playing the main role in the micturition related PAG functions.

\section{Studies featuring the function of the ventrolateral column of PAG}

Some studies have a top-down design, in such a way that, central electro-stimulation at PAG is accompanied by peripheral evaluation of function at bladder level. Electrical stimulation of the vlPAG elicits either contraction or inhibition of the bladder [37]. The optimum sites for evoking bladder contractions were located in and close to the laterodorsal tegmental nucleus (LDT)(which is the same structure functionally called PMC), and in the PAG, just dorsal or dorsolateral to the LDT, in rats [37]. Electrical stimulation of the ventral PAG elicits neuronal firing at the postganglionic nerves of the bladder [38]. Studies in cats confirm that, electrical or chemical (DL-homocysteine) stimulation of vlPAG, result in contraction of the bladder [17].

Inversely, some other studies have a down-top design, i.e. peripheral electro-stimulation is accompanied by central evaluation. Electrical stimulation of the pelvic nerves in the cat, evokes maximum field potentials in vlPAG [33]. Since the regions activated by the pelvic nerve stimulation differs from those activated by stimulation of the sensory pudendal or superficial perineal nerves, it is possible that, specific pathways exist for different nerve inputs to the PAG [33]. However, in another study, electrical stimulation of afferents in the pelvic nerve of the rat, evoked field potentials in the dorsal part of the PAG [38].

Single unit recordings in PAG columns either with or without stimulation of the bladder, derived some information regarding the PAG neuronal activity. The vlPAG shows three different patterns of neuronal firing rate during the micturition: increased firing rate, decreased firing rate, or no correlation with the micturition [39]. These neurons may correspond to specific functions they have during the micturition, some of them contracting the detrusor, some relaxing the sphincter, some receiving afferent sensory signals, and others unrelated to the micturition. A similar study accompanied by intravesical pressure recordings was conducted in cats [40]. However, only 16 out of 84 neurons that were recorded, were located in the PAG. The rest of the neurons were in adjacent midbrain areas. Nevertheless, this study emphasizes the existence of neurons in ventrolateral and lateral columns of the cat PAG, with firing patterns which change, corresponding to specific phases of micturition [40]. 
Chemical stimulation or suppression of the PAG exerts similar effects over the bladder. As detailed above, chemical stimulation of vlPAG increases the frequency of micturition in rats $[34,36]$ and contracts detrusor muscle in cat [17]. On the other hand, chemical inhibition of vlPAG attenuates the detrusor and EUS contractions in rats [35, 36]. Similarly, bilateral electrolytic lesion of vlPAG and IPAG in cat, attenuates detrusor contractions [40].

Neuronal activation may also be evaluated by specific markers such as c-Fos or NGF expression. Either anatomical manipulation or chemical irritation of the bladder may induce increased neuronal activity in the PAG. Increased c-Fos [41] or NGF [42] reactivity in vlPAG was observed after the induction of stress urinary incontinence by transabdominal urethrolysis in rats. Chemical bladder irritation, which is a painful stimulus, induced c-Fos expression in the PAG, though the exact PAG column was not stated [43].

\section{Studies featuring the function of the dorsolateral column of PAG}

Some studies proposed the possibility of the existence of a specific micturitionsuppressing region in dIPAG, acting via GABAergic inhibition of PMC [44]. Electrical stimulation of dIPAG elicits various types of reactions from the bladder. Electrical stimulation of the dorsal part of the PAG, including the dorsomedial and the dorsolateral columns, in rats, resulted a higher frequency of voiding, as well as some behavioural responses such as tense immobility, accompanied by exophthalmos and running and jumping responses [45]. A similar result, as bladder contraction, was encountered after electrical stimulation of points concentrated at the superior collicular and intercollicular levels, in an area involving the deep layers of the superior colliculus, the dIPAG, and the tegmental reticular formation, neighbouring the most lateral border of the PAG, in cat [46]. Nevertheless, this may be an erroneous conclusion due to inadvertent diffusion of electrical current to other PAG columns. In fact, electrical stimulation or glutamate microinjection within either ventrolateral, lateral or dorsolateral columns, evoked the rise of the intravesical pressure, as well as an increase of blood pressure [47]. Another reason behind this discrepancy between different studies may be the difference in craniocaudal location of the stimulation sites. For example, a similar study in cat mentioned rostral part of dorsal PAG and caudal part of ventral PAG, to be the main sites, at which high frequency electro-stimulation causes inhibition of micturition [40].

\section{Other functional studies}

Some other studies show that, stimulation of either the vl- or dIPAG would suppress the contraction of the bladder. Deep brain stimulation (DBS) in vlPAG, attenuates or completely suppresses the voiding in rats and humans [48]. Similar physiological activities such as rhythmic straining reflexes or defecation, alongside with micturition, are inhibited by electrical stimulation of the ventral or dorsal PAG, dorsal raphe nucleus and central tegmental field, with similar threshold intensities, in dogs [49]. These studies show that the electrical stimulation of various midbrain structures, and not only specific 
PAG columns, may suppress some pelvic functions. Such equivocal results may be due to a possible jamming effect over the normal electrical circuitry, contributing to the negative impact of DBS on micturition [34]. One possible explanation is the disruption of the normal coordinated voiding activity in the detrusor and sphincter muscles, after DBS.

Since the micturition reflex is under unconscious control during sleep, and nocturnal enuresis is a common associated disorder, it would be interesting to investigate the function of PAG during sleep, and the associated changes in detrusor contractions. Simultaneous recordings of the detrusor pressure, EUS electromyogram (EMG), cortical electroencephalogram (EEG), and single-unit activity in the PAG in rats reveals that, during slow wave EEG activity (SWA), voiding becomes more irregular and detrusor voiding pressure threshold, voiding volume threshold, and the duration of the bursting activity in the EUS EMG is raised, all in line with maintaining continence during sleep [50]. SWA is associated with slower neuronal firing rate in the PAG as well. Different sleep-like brain states are associated with changes in urodynamic properties, suggesting changing excitability of the micturition circuitry in the PAG. This may uncover some underlying factors in the pathophysiology of nocturnal enuresis [50].

In summary, the PAG receives ascending sensory signals from the bladder, and can modulate the bladder function by its descending efferent connections. Such bilateral connections may be important in conducting reflexive micturition in rodents, or the immature human. This comprises a feedback loop, with PAG continuously monitoring the state of bladder fullness, and induction of voiding in a supra-threshold filled bladder (Figure 2).

\section{The role of distinct cell groups in PAG function}

To better understand the organization of neural circuits, different cell populations contributing to various neural pathways are investigated (Table 1). The PAG, like most other brain regions, has a variety of distinct cell groups, and has immense connections with its cranially and caudally located CNS structures, related to the control of the micturition. Among these cell groups are dopaminergic, serotoninergic, glutamatergic and GABAergic neurons, and cells expressing neuronal nitric oxide synthase (nNOS). Dopamine, serotonin and glutamate are stimulatory neurotransmitters, and GABA and nNOS are inhibitory neurotransmitters. PAG neurons express receptors for all of these neurotransmitters (Table 1).

The vlPAG has distinct groups of glutamatergic cells which can stimulate other centers [51]. Chemogenetic or optogenetic stimulation of glutamatergic neurons in the vlPAG lead to detrusor contraction and voiding [52]. By contrast, chemogenetic or optogenetic activation of vlPAG GABAergic neurons delayed detrusor contraction and inhibited voiding [52]. The vlPAG GABAergic cells stimulated in this experiment, were most probably interneurons [53]. On the other hand, the main inhibitory GABAergic input to the vlPAG, relevant to the micturition, projects from dIPAG [44]. Glutamatergic cells of 
the vlPAG also control other important functions, including freezing [53] and nociception [54], which are controlled by two separate cell groups in the vlPAG [53]. Whether these cell groups are different from those vlPAG glutamatergic cells controlling voiding, or have some overlap, remains to be elucidated.

\section{Excitatory signalling}

In this section we mention glutamatergic, dopaminergic and serotoninergic neuronal signalling, arising from PAG and influencing micturition.

Glutamate serves as an excitatory neurotransmitter [55]. Its extracellular levels has been shown to be increased in PAG, after bladder distension or voiding, by microdialysis studies [56, 57]. Glutamatergic cells may project back to brainstem structures related to the control of the bladder contraction, to fulfil a micturition reflex, or to higher cortical regions to undergo further analysis and decision making. The vlPAG receives afferents from the lumbosacral cord [25] and has direct connections with the PMC [25], which then controls the micturition by its efferents to sacral parasympathetic segments. This circuit is especially very active in rodents and probably in the immature human infant, while higher decision-making brain centers are still underdeveloped. vlPAG glutamatergic cells probably stimulate the PMC, after receiving suprathreshold sensory signals from a full bladder. As a clinical correlate, multiple system atrophy (MSA), which is an extrapyramidal disease, has micturition symptoms including frequency, urgency, incontinence, or incomplete bladder emptying, as part of its manifestation. The number of glutamatergic cells in the ventrolateral, lateral, dorsomedial, and to a lesser extent dorsolateral PAG columns, were shown to be decreased in MSA [58].

The role of dopaminergic neurotransmission over micturition is controversial. Microinjection of SCH-23390, a dopamine antagonist, into the PAG, had contrary results over micturition in two different studies $[57,59]$. The PAG dopaminergic neurons mostly project rostrally to higher brain regions $[60,61]$. There is a loss of putative wake-active PAG dopaminergic neurons in patients suffering from either MSA or dementia with Lewy bodies, which may contribute to excessive daytime sleepiness in these disorders [62]. Both of these conditions have micturition problems. As micturition is in coordination with the sleep wake cycle, their possible role on micturition must be indirect, via influence over other autonomic systems.

Serotoninergic cells are abundant in the brain. The evidence regarding the role of serotonin in the regulation of micturition is very limited. Few serotoninergic neurons are present in the PAG, which are particularly scattered in vlAPG, close to the dorsal raphe nuclei. 


\begin{tabular}{|c|c|c|c|}
\hline & Synthesis ${ }^{1}$ & Receptor $^{1}$ & $\begin{array}{l}\text { Role in micturition (All experiments were performed on } \\
\text { rats) }\end{array}$ \\
\hline Dopamine & $\checkmark[133]$ & $\checkmark[134]$ & $\begin{array}{l}\text { Inhibitory : } \\
\text { - Application of a D1 receptor antagonist into the PAG } \\
\text { facilitated the micturition reflex [57] }\end{array}$ \\
\hline Serotonin & $\checkmark[135]$ & $\checkmark[136]$ & $\begin{array}{l}\text { No role detected so far: } \\
\text { - It seems to be responsible for nociception in the } \\
\text { PAG[56] }\end{array}$ \\
\hline Glutamate & $\checkmark[51]$ & $\checkmark[137]$ & $\begin{array}{l}\text { Excitatory: } \\
\text { - Chemogenetic or optogenetic stimulation of vlPAG } \\
\text { Glutamatergic neurons leads to voiding and } \\
\text { detrusor contraction [52]. } \\
\text { - Glutamatergic vlPAG cells were activated after } \\
\text { bladder electro-stimulation [73]. } \\
\text { - Glutamate microinjection within the PAG evoked a } \\
\text { rise of intravesical pressure [36, 47]. } \\
\text { - Saline infusion into the bladder with consequent } \\
\text { induction of the micturition reflex, resulted } \\
\text { increased extracellular glutamate levels in the PAG } \\
\text { [56] }\end{array}$ \\
\hline GABA & $\begin{array}{l}\checkmark[138, \\
139]\end{array}$ & $\begin{array}{l}\checkmark[140, \\
141]\end{array}$ & $\begin{array}{l}\text { Inhibitory: } \\
\text { - Chemogenetic or optogenetic activation of vlPAG } \\
\text { GABAergic neurons delays detrusor contraction and } \\
\text { inhibits voiding [52]. } \\
\text { - Microinjection of a GABA agonist into the vlPAG of } \\
\text { the rat depressed reflex voiding frequency, whereas } \\
\text { microinjection of a GABA antagonist into the same } \\
\text { region increased reflex voiding frequency [142] }\end{array}$ \\
\hline opioid & $\checkmark[143]$ & $\checkmark[144]$ & $\begin{array}{l}\text { Inhibitory: } \\
\text { - Injection of a } \mu \text { receptor agonist into the caudal } \\
\text { vlPAG abolished volume-evoked micturition [74]. } \\
\text { - Intracerebroventricular injection of morphine or a } \\
\mu \text {-agonist showed consistent inhibition of } \\
\text { spontaneous urinary bladder contractions [75]. }\end{array}$ \\
\hline
\end{tabular}

Table 1. Synthesis of neurotransmitters and the expression of their receptors in the PAG, and their functional significance. 1: Capability to synthesize and the bearing of specific receptors for a particular neurotransmitter implies the existence of efferent or afferent pathways incorporating that particular neurotransmitter in the PAG.

The mark $\checkmark$ positively denotes that, the chemical mediator identified, is either synthesized in the PAG, or the PAG bears receptors for them, in conjunction with their respective references.

This actually makes these few cell populations suspicious to be extra-raphe serotoninergic cells, not being part of the main PAG columns. There is not much known about the micturition related pathways within the PAG, involving serotonin for 
neurotransmission. The vlPAG serotoninergic cells inhibit the ejaculation in rats, and may contribute to SSRI (selective serotonin reuptake inhibitor) induced inhibition of ejaculation [63]. Yet, regulation of the autonomic function of the genital organs is different from that of the bladder. Since glutamate modulates 5-HT release in the PAG [64], and serotoninergic cells of the PAG also have projections toward other brainstem regions [65], there may be an indirect relation between the bladder stimulation and the serotonin system.

\section{Inhibitory signalling}

Inhibitory signalling from PAG arises from GABAergic cells, or neurons expressing nNOS.

GABAergic transmission has an indirect role over vlPAG, by internal connections via dorsolateral column of the PAG [11]. GABAergic cells of dlPAG project to vlPAG to suppress the micturition reflex [44]. PMC has reciprocal connections with the vIPAG [16, 17], and receives inhibitory GABAergic input from dlPAG [44](Figure 2). The existence of reciprocal connections between various columns of the PAG [11], emphasizes the existence of a micturition suppressing region in dIPAG.

The bladder's intramural ganglia, dorsal root ganglia, and spinal cord, contain nitric oxide (NO), the expression of which shows plasticity, following pathological lesions, such as pelvic nerve injury, chronic bladder irritation and urethral obstruction [66]. The rate of NO production in brain is dependent on dynamic regulation of its synthetic enzyme, nNOS [67]. There has been no report about the role of nNOS in the brain, related to the physiology of micturition. Most functions mediated by nNOS, including modulation of the cardiovascular, behavioural or nociceptive functions, have been found to be mainly active at dorsal PAG [68-70]. The existence of any possible role over the micturition pathways in the PAG by NO, would probably be mediated by its interactions with GABA [71]. Its inhibitory neuronal activity within the PAG [72] may also be explained accordingly, since GABA is an inhibitory neurotransmitter.

An overall comparison of various cell groups in a standardized setup, showes that in contrast to serotonergic, dopaminergic, GABAergic and nNOS synthesizing cells, only vlPAG glutamatergic neurons are activated upon receiving afferent bladder sensory signals [73].

Table 1. summarizes some key cell types in the PAG, bearing particular neurotransmitters, and their corresponding receptors, and describes their functions in micturition.

\section{Other important chemical mediators of PAG function}

Since the PAG is also a center for pain control, endogenous opioids have significant presence in this region. Their potential engagement in the control of voiding has been investigated in various ways. Among the different types of opioid receptors and PAG columns, it's only $\mu(\mathrm{mu})$ receptors which have inhibitory effect on the vlPAG, by abolishing volume-evoked micturition [74]. Intracerebroventricular injection of morphine or the $\mu$ agonist morphiceptin, confirms the aforementioned findings, showing 
consistent inhibition of spontaneous urinary bladder contractions [75]. The rapid onset of action and its limited distribution, shown by the intraventricular dye injection studies, indicate that its actions are confined predominantly to the periventricular and periaqueductal, or associated areas, and not the spinal cord [75].

The $\alpha 1$-adrenergic receptor antagonist Tamsulosin, and the PDE-5 inhibitor sildenafil, significantly suppressed the increase in neuronal activities measured by the expression of c-Fos and NGF, in the vIPAG, in an overactive bladder rat model [76]. Further evidence for the role of phosphodiesterase system comes from studies using caffeine. Caffeine is a methylxanthine alkaloid chemically related to the adenine and guanine bases of DNA. Its mechanism of action is by phosphodiesterase inhibition and adenosine antagonism. Caffeine administration to rats for 14 days increased bladder smooth muscle contraction pressure and time, determined by cystometry [77]. Expression levels of c-Fos and NGF in the vIPAG were also significantly increased following the administration of caffeine [77]. Hence, the phosphodiesterase system has possible regulatory role over the PAG, and can influence the micturition.

\section{Functional imaging of the PAG}

Functional imaging is a non-invasive tool for visualizing the activation of specific brain regions in response to various stimuli, respecting sensory afferent and motor efferent functioning. However, state of the art functional imaging techniques usually lack adequate spatial resolution to make reliable statements about the function of a specific column of the PAG ( figures $3 \mathrm{a}$ and $3 \mathrm{~b}$ ). Nevertheless, functional imaging studies have shed light on different connections of the PAG (figures 2 and $3 \mathrm{~b}$ ). They can also detect defects in some structural or functional pathologies involving the PAG. As the most common type of functional imaging, functional MRI (fMRI), is the imaging modality widely used to determine the activated brain regions in different phases of micturition.

Since PAG is also a center for handling nociceptive signals, it would be interesting to differentiate pain from other afferent sensory information processing. Both distention of the bladder and painful stimuli may activate the PAG, with different patterns. The vlPAG, among other regions, was activated in human PET scans after bladder distension, but not with intravesical ice bladder instillation, revealing different pathways in bladder distension and pain [78]. fMRI on healthy females shows that cold (pain) sensation is processed differently from bladder distension at the supraspinal level [79]. Parallel invivo studies in mice revealed that selective optogenetic activation of bladder sensory fibers, can differentially modulate nociceptive information and autonomic reflexes [80]. These findings show that, bladder nociceptive and mechanosensory signals have separate pathways, both in the periphery and in the brain.

PAG is also activated in some similarly related physiological processes, like pelvic muscle contraction or rectal distention. In a non-voiding model of voluntary micturition control, ventral pons and the PAG showed more enhanced activation patterns by fMRI, in 
voluntary contraction, than in the relaxation of the pelvic floor muscles [81]. Rectal distention in humans led to PAG activation detected by PET, that was also associated with increased heart rate and with increased plasma adrenaline [82].

The PAG is active in both storage and voiding phases of the micturition cycle, but the extent of its activity differs between these two phases. During the storage phase, the PAG is activated, but the PMC is inactive, and during the voiding, the PMC maintains activation, and the activation of the PAG enhances [83].

Hereby, more evidence is provided supporting the activity of the PAG in storage and voiding phases of micturition.

\section{The PAG activation during the storage phase:}

These experiments are usually designed in such a way that, the brain would be scanned, while the bladder is being passively filled by intravesical infusion of saline. This way, mechanosensory signals arising from an expanding bladder would reach central micturition control centers. The activity in the right anterior insula and the PAG in human was enhanced at higher bladder volumes, in a non-voiding bladder, detected by fMRI [84](figure 3b) and PET [85]. PAG activation after bladder filling is accompanied by the activation of the inferior parietal lobule, as well as the right insula and the dorsal anterior cingulate cortex (ACC)[86]. Passive filling and emptying of the bladder induces PAG activation as well [87-89]. Studies on Parkinson's disease (PD) patients show that the activity of the PAG is enhanced in a full bladder, compared to an empty bladder [90]. Furthermore, DBS of subthalamic nucleus increases the PAG activity in PD patients [90]. This would indirectly influence the activity of other cortical regions related to micturition, and ultimately restore afferent bladder information processing [90]. These findings point to the fact that, the PAG processes the sensory signals derived from a filling bladder. These signals may then flow to specific cortical regions and reach the awareness.

\section{The PAG activation during the Voiding phase:}

\section{Initiation of voiding}

There is a special role for the PAG in the initial moments of the bladder emptying. The PAG is consistently active during "attempted micturition"[84](figure 3b). During this maneuver, the individuals direct their attention to the sensations arising from the bladder and urethra, and increase the desire to void as if initiating to allow the urine to pass [84]. Initiation of voiding induced significant activity in cortical regions, in addition to the PAG, as shown by fMRI, whereas actual micturition was associated with significantly less such activity [84]. Unsuccessful attempts at micturition result from inefficient activation of the PAG and PMC during the initiation phase, which itself may be secondary to inadequate antecedent activation of frontoparietal and cingulate cortices, involved in decision-making for the micturition behaviour [91]. 


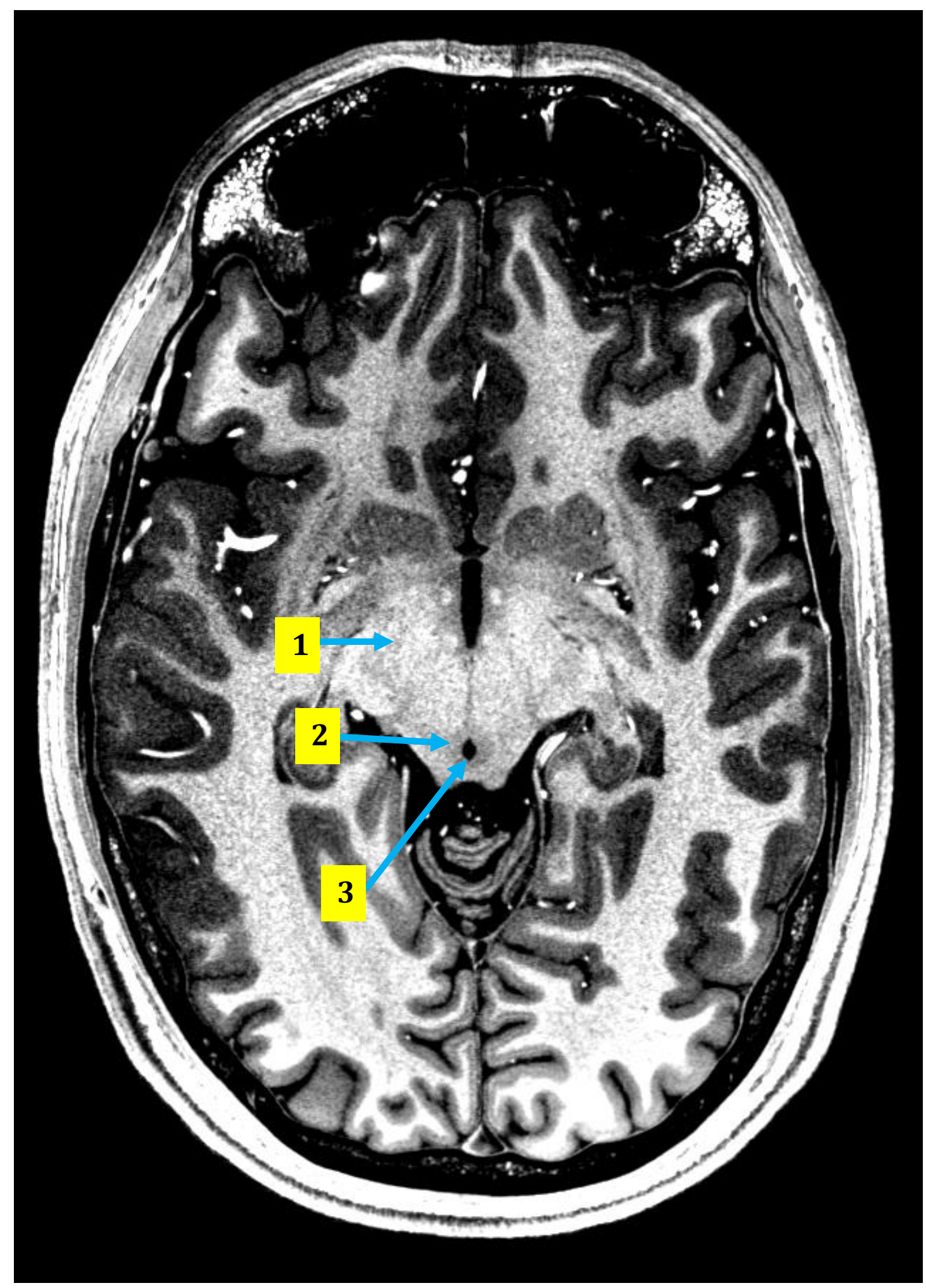

Figure 3A 


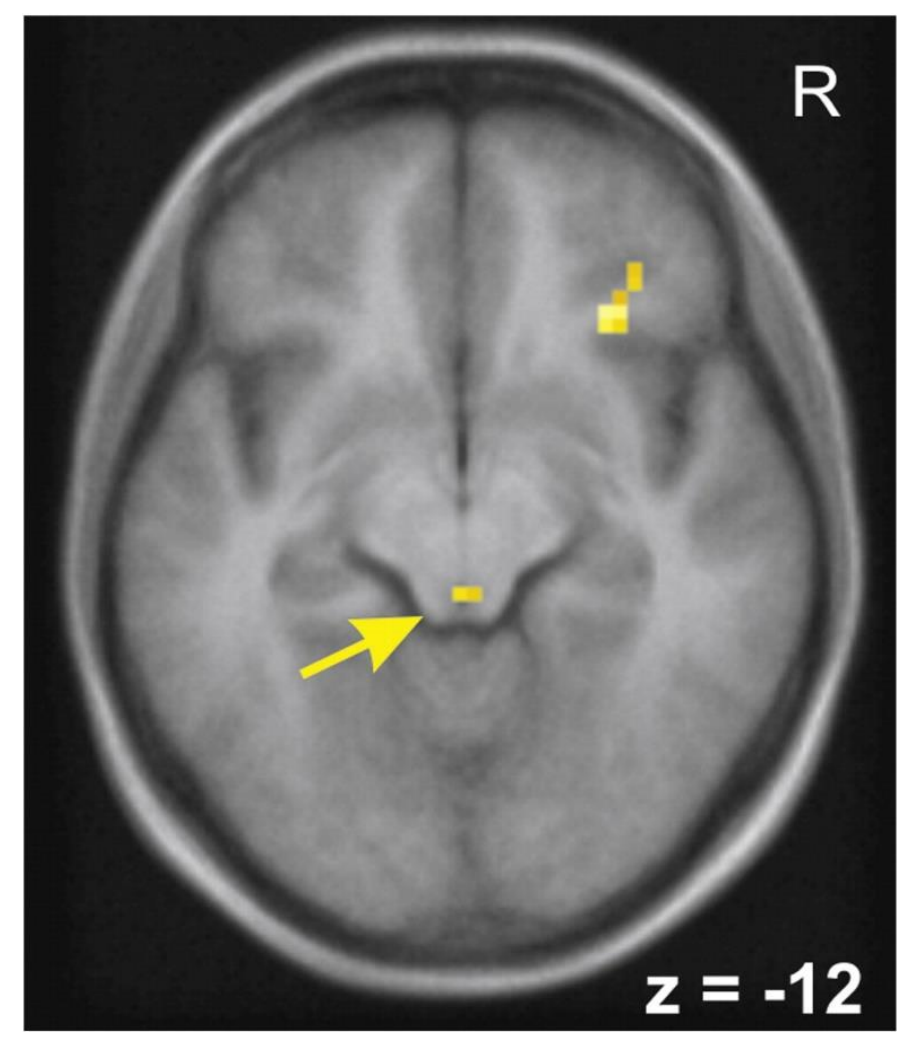

Figure 3B

\section{Figure 3.}

A. A transverse section through human midbrain in a normal subject, showing the cerebral peduncles (1), the PAG (2) and the aqueduct (3) (7 Tesla MRI)[131, 132].

B. An fMRI showing the activated regions in a transverse section of the human brain. The PAG (arrow) along with the right insula, show more activity in a full bladder rather than an empty bladder, during attempted micturition (1.5 Tesla fMRI)(reprinted with permission from Elsevier)[84].

The same results can be found by PET scans [92]. fMRI studies found out that imitation or interruption of voiding by relaxation or contraction of the pelvic floor muscles in healthy women, could induce activation patterns in the PAG, cortical regions, and some other micturition control centers [93]. The above findings emphasize the role of the PAG in the integration and relay of information coming from different areas (such as the spinal cord and the cortico-limbic system), which are essential in micturition physiology. This signifies the role of the PAG, to be the trigger for implementing the decision for voiding.

Analysis of reflexive micturition in healthy rats by animal fMRI showed the activation of the PAG[94]. High resolution animal fMRI in rabbit could specifically identify the vlPAG, for having an important role in bladder dysfunction biofeedback[95]. This is confirmed by a PET study in humans also showing the vlPAG to be activated after distention of the bladder[78]. Columnar differentiation in human PAG could also be done by a PET scan, which identified the activation of the tegmental part of the PAG, denoting either ventrolateral or lateral columns, during micturition[96]. Nonspecific involvement of the PAG was mentioned in some other human PET scans[97, 98]. These discoveries are 
corroborated by a single photon emission computed tomography (SPECT) in human, showing increased PAG activation during voiding[99].

With upcoming research by modern 7 Tesla and 9 Tesla MRI machines, we shall gain more precise information regarding the connections of individual PAG columns with specific parts of the brain.

\section{Connectivity analysis}

It is often helpful to investigate the connections between one particular structure and other brain regions, and their temporal pattern of activation. The PAG has been found to be functionally connected with some other brain regions during voiding (figure 2). The physiophysiological interaction(PPI) is a sophisticated tool used to elucidate the effective connectivity between different brain regions and a particular task, i.e. the micturition. It is generally based on observing alterations in the slope of the regression line of two different brain regions, with respect to another region, or a particular task[100]. This method aids in revealing the cortical pathways, together with their associated functions (figure 2).

fMRI signals of the right insula/operculum, change in parallel with PAG activity (indicating connectivity), when the subject perceives an enhanced urge to void during "attempted micturition" [84]. Indeed, positive functional connectivity exists between the vlPAG and brain centers involved in micturition, such as the ACC or insula, shown by resting state fMRI (rsfMRI)[101]. The PAG clearly shows some laterality in its effective connectivity, which is to right insula during the filling of the bladder[102, 103].

White matter hyperintensities (WMH) are common in the older population, and have been linked to demyelination, spongiosis, and glial proliferation, presumably after lacunar ischemic infarcts. Global WMH has been linked to impairments of mobility, cognition, affect, and continence[104]. These hyperintensities could be located in any of the central micturition control centers, and are particularly related to the severity of the incontinence[105]. The PAG is one of the regions, of which activity shows positive correlation with the global WMH[106]. Hence PAG may be secondarily affected by WMH, contributing to the micturition symptoms.

The imaging modalities explained above may be used for experimental or diagnostic purposes. Putting various cortical connections of the PAG together, we find a circuit of continuous monitoring of the level of the bladder fullness, vigilance of the environmental circumstances, and deciding to void at the right moment. This comprises a cortical feedback loop, completed by incorporating the ventrolateral and dorsolateral PAG columns, for thinking and implementation of the decisions (Figure 2).

\section{PAG in neuro-pathological conditions and their effects on micturition.}

The PAG may be affected in several pathological states, and its role has been investigated in various disorders showing lower urinary tract problems as part of their spectrum of 
symptoms. Here, we explain some structural and functional disorders, affecting the PAG and compromising micturition.

\section{Structural pathologies}

The so far reported structural pathologies include stroke, neoplastic lesions, hydrocephalus and spinal cord injury. Spinal cord injury would secondarily alter PAG activity states, as discussed below.

Structural damage involving the PAG has been reported to be a cause of voiding problems. Cerebral stroke, which is a debilitating disorder in the elderly, may cause voiding dysfunction. Urinary retention was among the manifestations of a patient suffering from acute ischemic stroke of the brainstem[107]. Diffusion-weighted MRI showed hyperintensity over the left paramedian segment of the lower midbrain, which includes the PAG. The computed tomography also showed a hemorrhagic transformation and intraventricular hemorrhage in the fourth ventricle[107], which is directly beneath the aqueduct. Either indirect ischemic damage of the PAG or chemical irritation by a hemorrhagic cerebrospinal fluid (CSF) inside the aqueduct, could explain the micturitionrelated symptomatology. In an intracerebral hemorrhage (ICH) induced rat model of neurogenic voiding dysfunction, c-Fos and NGF expression levels in the neuronal voiding centers, including vlPAG, were significantly increased with induced ICH, as compared to the control rats[108].

Various other structural defects may affect the PAG and lead to micturition problems. We here mention two case reports of subjects with neoplastic lesions or hydrocephalus, which compromised normal micturition by affecting the PAG. A lesion in the midbrain and upper pons was detected by MRI scan of a 34-year-old man presenting with a history of delayed ejaculation, voiding difficulties and diplopia, which was a developmental abnormality such as a dermoid or teratoma[109]. In evaluation of patients with possible idiopathic normal pressure hydrocephalus(iNPH) with typical imaging features (ventricular enlargement) and normal CSF pressure, a small bladder capacity and detrusor overactivity was seen in $95 \%$ of patients[110]. Since the PAG completely encircles the aqueduct, there's a possibility that, this structure would be affected by minor dimensional changes in iNPH[110].

Sacral neuromodulation is commonly used in various neurologically mediated continence problems. The bladder filling and rest contrast, in eight spinal cord injured (SCI) participants, elicited clear activation, measured by fMRI, in the PAG and in a continuous area in the right midbrain[87]. Following two weeks of pudendal stimulation treatment, abnormal PAG overactivity were decreased in all six participants, as well as in the four clinically improved subjects. Thus the PAG may be overactive in the SCI group, following the sudden loss of the spinal afferent inputs[87]. The vlPAG overactivity in the SCI subjects was also demonstrated in a rat model, by increased expression of c-Fos or NGF, relative to the sham operated group[111]. Abnormal PAG function would be 
restored to normal by sacral neuromodulation, in individuals with urinary retention[112].

\section{Functional pathologies}

These disorders encompass demyelinating diseases, PD, MSA, migraine, Wernicke's encephalopathy, nocturnal enuresis and urge incontinence, which will be elaborated below.

One of the most common demyelinating diseases is multiple sclerosis (MS), a common debilitating disorder with white matter plaques affecting any part of the brain. In $18.7 \%$ of MS patients, lesions were located in the PAG[113]. 36\% of these lesions were periventricular lesions of the third ventricle, extending towards the aqueduct. Bowel and bladder disability scores in MS patients are correlated with the volume of lesions in the medial frontal lobes, cerebellum, insula, dorsal midbrain including dorsal part of the PAG, and pons, areas known to be involved in the control of micturition[114]. As a clinical correlate, a 31 year old man had suffered from sudden voiding difficulty and retention. A filling cystometrogram revealed an atonic bladder with diminished bladder sensation. Hyperintensities were shown in the PAG in $\mathrm{T}_{2} \mathrm{~W}$-MRI, that were reduced after steroid therapy, with subsequent improvement of the voiding symptoms. He was suspected to have a demyelinating disease such as MS[115].

Patients with PD, which is an extrapyramidal disorder, may have lower urinary tract symptoms. Elimination of dopaminergic neurons by 6-OHDA microinjection into the PAG in a rat model of $\mathrm{PD}$, leads to altered micturition patterns[116]. Moreover, the reduction of the amplitude of the evoked potentials measured in the PAG, elicited by means of electrical stimulation of the pelvic nerve in the rat, is more pronounced in PD animals compared to sham animals, after the intravenous administration of an adenosine receptor antagonist [117]. Besides the direct effect dopaminergic lesions can have on the PAG, there is some evidence showing that the micturition problems in PD may be a consequence of a primary problem residing in the substantia nigra ( $\mathrm{SN}$ ), secondarily affecting the PAG by its projections toward the PAG. Increased c-Fos reactivity was observed in the PAG and ACC, in a PD rat model induced by 6-hydroxydopamine injection into SN[118]. Moreover, a 6-OHDA lesion in the SN produces a transient increase in voiding frequency within the first two weeks, with recovery of urinary function by four weeks post-lesion[119]. In PD patients, significant brain activation can be detected by PET in the PAG, during detrusor overactivity (measured by intravesical pressure monitoring)[120]. As another example of extrapyramidal disease, MSA, which includes micturition symptoms, shows neurochemical changes in the PAG (detailed above)[58].

Furthermore, a possible role of the PAG has been suggested in nocturnal enuresis. Singleunit activity in the lateral and ventrolateral columns of the PAG were linked to the occurrence of voids induced by continuous infusion of saline into the bladder of urethane anesthetized rats, to mimic sleep-like brain states[50]. Almost a quarter of the recorded neurons were responsive during the micturition reflex. Their spontaneous firing rate in the absence of bladder stimuli, decreased during slow wave EEG states[50]. This suggests 
that the micturition reflex is reset centrally during the sleep. Failure of this mechanism could contribute to the development of nocturnal enuresis[121].

Various other functional disorders may have PAG malfunction together with micturition symptoms. Vegetative symptoms including increased micturition, may occur in migraine[122]. More specifically, vlPAG was found in a PET study, as a structure that may be implicated in migraine pathophysiology[123]. PAG dysfunction also has been shown in Wernicke's encephalopathy[124]. A case report of Wernicke's encephalopathy in a pregnant woman described decreased bladder volume and detrusor hyperreflexia in urodynamic studies. Brain MRI revealed abnormal intensities in medial thalamichypothalamic regions, and the PAG[124]. PAG[125] or midbrain[126] activation were shown by fMRI studies, in urge incontinent patients as well. rCBF analysed by PET is decreased in midbrain during sacral neuromodulation in chronically implanted urge incontinent patients[127].

By its involvement in a multitude of disorders leading to micturition problems, the PAG must be included in future diagnostic or therapeutic algorithms concerning neurological causes of the bladder dysfunction. Specifically, with the availability of future high precision MRI machines, the PAG can be more specifically evaluated, for diagnostic purposes.

\section{Conclusion}

The PAG plays the role of a switchboard located in the brainstem, coordinating the evolutionary primitive and advanced brain centers. It has a broad spectrum of functions and has a paramount role in the control of micturition. The PAG functions as a sensory and motor relay station for the ascending afferents from the lower urinary tract, and descending afferents from the cortical areas. This is partly made possible by its special position at the intersection of the forebrain and the hindbrain. Two of the four columns of the PAG, namely the ventrolateral and dorsolateral columns, demonstrate more significant involvement in this respect. The vlPAG is more connected to the caudal, and the dIPAG with cranial structures of the CNS[128]. The intercolumnar connections[11] traverse the information between vlPAG and dlPAG, and thus complete a full circuit. Pathological conditions affecting the PAG may compromise the continence, and some of them may be detected by modern imaging techniques. Thus, the PAG will be a potential diagnostic and therapeutic target for specific incontinence problems and voiding dysfunctions. This may be done pharmacologically, by for example targeting its glutamatergic neurotransmission, or surgically, by DBS of particular PAG columns.

\section{Acknowledgement}

We would like to appreciate Geertjan van Zonneveld for assistance in preparation of the schematic diagram (figure 2), and Mathijs M. de Rijk for extracting the normal brain MRI from the database (Figure 3a), both from Maastricht University. 


\section{REFERENCES}

1. Rathner, J.A. and S.F. Morrison, Rostral ventromedial periaqueductal gray: a source of inhibition of the sympathetic outflow to brown adipose tissue. Brain Res, 2006. 1077(1): p. 99-107.

2. Farkas, E., A.S. Jansen, and A.D. Loewy, Periaqueductal gray matter input to cardiac-related sympathetic premotor neurons. Brain Res, 1998. 792(2): p. 179-92.

3. Mouton, L.J. and G. Holstege, The Periaqueductal Gray in the Cat Projects to Lamina-Viii and the Medial Part of Lamina-Vii Throughout the Length of the Spinal-Cord. Experimental Brain Research, 1994. 101(2): p. 253-264.

4. Budai, D., I. Harasawa, and H.L. Fields, Midbrain periaqueductal gray (PAG) inhibits nociceptive inputs to sacral dorsal horn nociceptive neurons through alpha(2)-adrenergic receptors. Journal of Neurophysiology, 1998. 80(5): p. 2244-2254.

5. Barrington, F.J.F., THE EFFECT OF LESIONS OF THE HIND- AND MID-BRAIN ON MICTURITION IN THE CAT. Quarterly Journal of Experimental Physiology, 1925. 15(1): p. 81-102.

6. Bandler, R. and M.T. Shipley, Columnar organization in the midbrain periaqueductal gray: modules for emotional expression? Trends Neurosci, 1994. 17(9): p. 379-89.

7. Parvizi, J., G.W. Van Hoesen, and A. Damasio, Selective pathological changes of the periaqueductal gray matter in Alzheimer's disease. Annals of Neurology, 2000. 48(3): p. 344-353.

8. Benarroch, E.E., Periaqueductal gray: an interface for behavioral control. Neurology, 2012. 78(3): p. 210-7.

9. Linnman, C., et al., Neuroimaging of the periaqueductal gray: state of the field. Neuroimage, 2012. 60(1): p. 505-22.

10. Carrive, P. and M.M. Morgan, Chapter 10 - Periaqueductal Gray A2 - Mai, Jürgen K, in The Human Nervous System (Third Edition), G. Paxinos, Editor. 2012, Academic Press: San Diego. p. 367-400.

11. Jansen, A.S.P., et al., Local connections between the columns of the periaqueductal gray matter: a case for intrinsic neuromodulation (vol 784, pg 329, 1998). Brain Research, 1998. 797(2): p. 368-368.

12. Griffiths, D.J., Use of functional imaging to monitor central control of voiding in humans. Handb Exp Pharmacol, 2011(202): p. 81-97.

13. Griffiths, D., Chapter 7 - Functional imaging of structures involved in neural control of the lower urinary tract, in Handbook of Clinical Neurology, B.V. David and B. François, Editors. 2015, Elsevier. p. 121-133.

14. Mtui, E., G. Gruener, and P. Dockery, reticular formation, in Fitzgerald's Clinical Neuroanatomy and Neuroscience

2015, Elsevier.

15. Griffiths, D., Neural control of micturition in humans: a working model. Nat Rev Urol, 2015. 12(12): p. 695-705.

16. Kuipers, R., L.J. Mouton, and G. Holstege, Afferent projections to the pontine micturition center in the cat. Journal of Comparative Neurology, 2006. 494(1): p. 36-53.

17. Taniguchi, N., et al., A study of micturition inducing sites in the periaqueductal gray of the mesencephalon. J Urol, 2002. 168(4 Pt 1): p. 1626-31. 
18. Marson, L., Identification of central nervous system neurons that innervate the bladder body, bladder base, or external urethral sphincter of female rats: A transneuronal tracing study using pseudorabies virus. Journal of Comparative Neurology, 1997. 389(4): p. 584-602.

19. Minor L, S.S., DonCarlos L, estrogen receptors in central nervous system micturition pathways in a rat model, in International Continence Society. paris.

20. Ding, Y.-Q., et al., Direct projections from the medial preoptic area to spinally-projecting neurons in Barrington's nucleus: an electron microscope study in the rat. Neuroscience Letters, 1999. 271(3): p. 175-178.

21. Rickey, L.M., S. Sarkey, and L.L. DonCarlos, Estrogen-sensitive projections from the medial preoptic area to the dorsal pontine tegmentum, including Barrington's nucleus, in the rat. Neurourol Urodyn, 2008. 27(5): p. 440-5.

22. Rizvi, T.A., M. Ennis, and M.T. Shipley, Reciprocal Connections between the Medial Preoptic Area and the Midbrain Periaqueductal Gray in Rat - a Wga-Hrp and Pha-L Study. Journal of Comparative Neurology, 1992. 315(1): p. 1-15.

23. Simerly, R.B. and L.W. Swanson, Projections of the medial preoptic nucleus: a Phaseolus vulgaris leucoagglutinin anterograde tract-tracing study in the rat. J Comp Neurol, 1988. 270(2): p. 209-42.

24. Marson, L. and K.A. Foley, Identification of neural pathways involved in genital reflexes in the female: a combined anterograde and retrograde tracing study. Neuroscience, 2004. 127(3): p. 723-36.

25. Ding, Y.Q., et al., Direct projections from the lumbosacral spinal cord to Barrington's nucleus in the rat: a special reference to micturition reflex. J Comp Neurol, 1997. 389(1): p. 149-60.

26. Klop, E.M., et al., Neurons in the lateral sacral cord of the cat project to periaqueductal grey, but not to thalamus. Eur J Neurosci, 2005. 21(8): p. 2159-66.

27. Kuipers, R. and E.M. Klop, Neurons in the guinea pig (Cavia porcellus) lateral lumbosacral spinal cord project to the central part of the lateral periaqueductal gray matter. Brain Res, 2006. 1101(1): p. 43-50.

28. Mtui, E., G. Gruener, and P. Dockery, Autonomic Nervous System, in Fitzgerald's Clinical Neuroanatomy and Neuroscience E-Book. 2015, Elsevier Health Sciences. p. 145.

29. Blok, B.F., H. De Weerd, and G. Holstege, Ultrastructural evidence for a paucity of projections from the lumbosacral cord to the pontine micturition center or M-region in the cat: a new concept for the organization of the micturition reflex with the periaqueductal gray as central relay. J Comp Neurol, 1995. 359(2): p. 300-9.

30. Mouton, L.J. and G. Holstege, Segmental and laminar organization of the spinal neurons projecting to the periaqueductal gray (PAG) in the cat suggests the existence of at least five separate clusters of spino-PAG neurons. J Comp Neurol, 2000. 428(3): p. 389-410.

31. Mouton, L.J., V.G.J.M. VanderHorst, and G. Holstege, Large segmental differences in the spinal projections to the periaqueductal gray in the cat. Neuroscience Letters, 1997. 238(12): p. 1-4.

32. Mitsui, T., et al., Chemical bladder irritation provokes c-fos expression in the midbrain periaqueductal gray matter of the rat. Brain Res, 2003. 967(1-2): p. 81-8.

33. Duong, M., J.W. Downie, and H.J. Du, Transmission of afferent information from urinary bladder, urethra and perineum to periaqueductal gray of cat. Brain Research, 1999. 819(12): p. 108-119.

34. Stone, E., J.H. Coote, and T.A. Lovick, Effect of electrical vs. chemical deep brain stimulation at midbrain sites on micturition in anaesthetized rats. Acta Physiologica, 2015. 214(1): p. 135-145. 
35. Matsuura, S., G.V. Allen, and J.W. Downie, Volume-evoked micturition reflex is mediated by the ventrolateral periaqueductal gray in anesthetized rats. Am J Physiol, 1998. 275(6 Pt 2): p. R2049-55.

36. Matsuura, S., J.W. Downie, and G.V. Allen, Micturition evoked by glutamate microinjection in the ventrolateral periaqueductal gray is mediated through Barrington's nucleus in the rat. Neuroscience, 2000. 101(4): p. 1053-1061.

37. Noto, H., et al., Excitatory and inhibitory influences on bladder activity elicited by electrical stimulation in the pontine micturition center in the rat. Brain Res, 1989. 492(1-2): p. 99115.

38. Noto, H., et al., Electrophysiological Analysis of the Ascending and Descending Components of the Micturition Reflex Pathway in the Rat. Brain Research, 1991. 549(1): p. 95-105.

39. MATSUMOTO Kengo, S.T., YAMAMOTO Gen Characterization of micturition-related single neuron activities and their distribution in periaqueductal gray matter (PAG) in anesthetized rats. J Pharmacol Sci 2006. 100: p. 251.

40. Liu, Z., et al., Micturition-related neuronal firing in the periaqueductal gray area in cats. Neuroscience, 2004. 126(4): p. 1075-82.

41. Ko, I.G., et al., Effect of Treadmill Exercise on Leak-point pressure and Neuronal Activation in Brain of Rats with Stress Urinary Incontinence. Int Neurourol J, 2010. 14(3): p. 141-8.

42. Ko, I.G., et al., Swimming: effects on stress urinary incontinence and the expression of nerve growth factor in rats following transabdominal urethrolysis. Int Neurourol J, 2011. 15(2): p. 74-81.

43. Zhi L, Y.L., Libing M, Dan W, Limin W, Jun M, Tomoyuki U, Ryuji S, the different relationship with nerve center from normal micturition to morbid micturition - comparing between normal and cystitis model rat by using c-fos method in International Continence Society. beijing.

44. Numata, A., et al., Micturition-suppressing region in the periaqueductal gray of the mesencephalon of the cat. American Journal of Physiology-Regulatory Integrative and Comparative Physiology, 2008. 294(6): p. R1996-R2000.

45. Vargas, L.C., T.D. Marques, and L.C. Schenberg, Micturition and defensive behaviors are controlled by distinct neural networks within the dorsal periaqueductal gray and deep gray layer of the superior colliculus of the rat. Neuroscience Letters, 2000. 280(1): p. 45-48.

46. Koyama, Y., A. Makuya, and M. Kuru, Vesico-motor areas in the cat midbrain. Jpn J Physiol, 1962. 12: p. 63-80.

47. Rocha, I., G. Burnstock, and K.M. Spyer, Effect on urinary bladder function and arterial blood pressure of the activation of putative purine receptors in brainstem areas. Autonomic Neuroscience-Basic \& Clinical, 2001. 88(1-2): p. 6-15.

48. Green, A.L., et al., Switching Off Micturition Using Deep Brain Stimulation at Midbrain Sites. Annals of Neurology, 2012. 72(1): p. 144-147.

49. Fukuda, H. and T. Koga, Midbrain stimulation inhibits the micturition, defecation and rhythmic straining reflexes elicited by activation of sacral vesical and rectal afferents in the dog. Exp Brain Res, 1991. 83(2): p. 303-16.

50. Crook, J. and T. Lovick, Urodynamic function during sleep-like brain states in urethane anesthetized rats. Neuroscience, 2016. 313: p. 73-82.

51. Barbaresi, P., G. Gazzanelli, and M. Malatesta, Glutamate-positive neurons and terminals in the cat periaqueductal gray matter (PAG): a light and electron microscopic immunocytochemical study. J Comp Neurol, 1997. 383(3): p. 381-96. 
52. Verstegen, H., et al., Brain Networks Controlling Bladder Filling and Voiding. Journal of Urology, 2017. 197(4): p. E1150-E1150.

53. Tovote, P., et al., Midbrain circuits for defensive behaviour. Nature, 2016. 534(7606): p. 206-12.

54. Samineni, V.K., et al., Divergent Modulation of Nociception by Glutamatergic and GABAergic Neuronal Subpopulations in the Periaqueductal Gray. eNeuro, 2017. 4(2).

55. Clements, J.R., et al., Localization of glutamate, glutaminase, aspartate and aspartate aminotransferase in the rat midbrain periaqueductal gray. Exp Brain Res, 1987. 67(3): p. 594-602.

56. Kitta, T., et al., Differences in neurotransmitter systems of ventrolateral periaqueductal gray between the micturition reflex and nociceptive regulation: An in vivo microdialysis study. Int J Urol, 2016. 23(7): p. 593-8.

57. Kitta, T., et al., GABAergic mechanism mediated via D1 receptors in the rat periaqueductal gray participates in the micturition reflex: an in vivo microdialysis study. European Journal of Neuroscience, 2008. 27(12): p. 3216-3225.

58. Benarroch, E.E., et al., Differential involvement of the periaqueductal gray in multiple system atrophy. Auton Neurosci, 2010. 158(1-2): p. 111-7.

59. STONE, E., MIDBRAIN CONTROL OF MICTURITION IN THE RAT chapter 5 phd thesis. 2012: University of Birmingham.

60. Lu, J., T.C. Jhou, and C.B. Saper, Identification of wake-active dopaminergic neurons in the ventral periaqueductal gray matter. Journal of Neuroscience, 2006. 26(1): p. 193-202.

61. Li, S., Y. Shi, and G.J. Kirouac, The hypothalamus and periaqueductal gray are the sources of dopamine fibers in the paraventricular nucleus of the thalamus in the rat. Front Neuroanat, 2014. 8: p. 136.

62. Benarroch, E.E., et al., Dopamine cell loss in the periaqueductal gray in multiple system atrophy and Lewy body dementia. Neurology, 2009. 73(2): p. 106-112.

63. Normandin, J.J. and A.Z. Murphy, Serotonergic lesions of the periaqueductal gray, a primary source of serotonin to the nucleus paragigantocellularis, facilitate sexual behavior in male rats. Pharmacology Biochemistry and Behavior, 2011. 98(3): p. 369-375.

64. Maione, S., et al., Metabotropic glutamate receptors modulate serotonin release in the rat periaqueductal gray matter. Naunyn-Schmiedebergs Archives of Pharmacology, 1998. 358(4): p. 411-417.

65. Beitz, A.J., R.D. Shepard, and W.E. Wells, The periaqueductal gray-raphe magnus projection contains somatostatin, neurotensin and serotonin but not cholecystokinin. Brain Research, 1983. 261(1): p. 132-137.

66. Zhou, Y. and E.A. Ling, Neuronal nitric oxide synthase in the neural pathways of the urinary bladder. J Anat, 1999. 194 ( Pt 4): p. 481-96.

67. Zhou, L. and D.Y. Zhu, Neuronal nitric oxide synthase: structure, subcellular localization, regulation, and clinical implications. Nitric Oxide, 2009. 20(4): p. 223-30.

68. Ishide, T., et al., Nitric oxide within periaqueductal gray modulates glutamatergic neurotransmission and cardiovascular responses during mechanical and thermal stimuli. Neuroscience Research, 2005. 51(1): p. 93-103.

69. Carvalho-Netto, E.F., et al., Role of glutamate NMDA receptors and nitric oxide located within the periaqueductal gray on defensive behaviors in mice confronted by predator. Psychopharmacology, 2009. 204(4): p. 617-625. 
70. Nunes-de-Souza, R.L., et al., Role of nitric oxide in the periaqueductal gray in defensive behavior in mice: influence of prior local $N$-methyl-D-aspartate receptor activation and aversive condition. Psychology \& Neuroscience, 2010. 3: p. 59-66.

71. Xing, J., D.P. Li, and J. Li, Role of GABA receptors in nitric oxide inhibition of dorsolateral periaqueductal gray neurons. Neuropharmacology, 2008. 54(4): p. 734-44.

72. Lovick, T.A. and B.J. Key, Inhibitory effect of nitric oxide on neuronal activity in the periaqueductal grey matter of the rat. Exp Brain Res, 1996. 108(3): p. 382-8.

73. Zare, A., et al., Glutamatergic cells in the periaqueductal gray matter mediate sensory inputs after bladder stimulation in freely moving rats. Int J Urol, 2018.

74. Matsumoto, S., et al., Activation of mu opioid receptors in the ventrolateral periaqueductal gray inhibits reflex micturition in anesthetized rats. Neuroscience Letters, 2004. 363(2): p. 116-119.

75. Dray, A. and R. Metsch, Opioid receptor subtypes involved in the central inhibition of urinary bladder motility. Eur J Pharmacol, 1984. 104(1-2): p. 47-53.

76. Kim, S.E., et al., An animal study to compare the degree of the suppressive effects on the afferent pathways of micturition between tamsulosin and sildenafil. Journal of Biomedical Science, 2013. 20.

77. Cho, Y.S., et al., Caffeine enhances micturition through neuronal activation in micturition centers. Molecular Medicine Reports, 2014. 10(6): p. 2931-2936.

78. Matsuura, S., et al., Human brain region response to distention or cold stimulation of the bladder: A positron emission tomography study. Journal of Urology, 2002. 168(5): p. 20352039.

79. Mehnert, U., et al., The Supraspinal Neural Correlate of Bladder Cold Sensation-An fMRI Study. Human Brain Mapping, 2011. 32(6): p. 835-845.

80. DeBerry, J.J., et al., Differential Regulation of Bladder Pain and Voiding Function by Sensory Afferent Populations Revealed by Selective Optogenetic Activation. Front Integr Neurosci, 2018. 12: p. 5.

81. Seseke, S., et al., Gender differences in voluntary micturition control - An fMRI study. Neuroimage, 2008. 43(2): p. 183-191.

82. Suzuki, H., et al., Brain activation associated with changes in heart rate, heart rate variability, and plasma catecholamines during rectal distention. Psychosom Med, 2009. 71(6): p. 619-26.

83. Tai, C.F., et al., Brain Switch for Reflex Micturition Control Detected by fMRI in Rats. Journal of Neurophysiology, 2009. 102(5): p. 2719-2730.

84. Kuhtz-Buschbeck, J.R., et al., Control of bladder sensations: An fMRI study of brain activity and effective connectivity. Neuroimage, 2009. 47(1): p. 18-27.

85. Athwal, B.S., et al., Brain responses to changes in bladder volume and urge to void in healthy men. Brain, 2001. 124: p. 369-377.

86. Griffiths, D.J., et al., Cerebral control of the lower urinary tract: How age-related changes might predispose to urge incontinence. Neuroimage, 2009. 47(3): p. 981-986.

87. Zempleni, M.Z., et al., Cortical substrate of bladder control in SCI and the effect of peripheral pudendal stimulation. Neuroimage, 2010. 49(4): p. 2983-2994.

88. Griffiths, D., et al., Brain control of normal and overactive bladder. Journal of Urology, 2005. 174(5): p. 1862-1867.

89. Tadic, S.D., et al., Brain responses to bladder filling in older women without urgency incontinence. Neurourology and Urodynamics, 2013. 32(5): p. 435-440. 
90. Herzog, J., et al., Improved sensory gating of urinary bladder afferents in Parkinson's disease following subthalamic stimulation. Brain, 2008. 131(Pt 1): p. 132-45.

91. Michels, L., et al., Supraspinal Control of Urine Storage and Micturition in Men--An fMRI Study. Cereb Cortex, 2015. 25(10): p. 3369-80.

92. Takao, T., et al., Brain responses during the first desire to void: A positron emission tomography study. International Journal of Urology, 2008. 15(8): p. 724-728.

93. Seseke, S., et al., Voluntary pelvic floor muscle control--an fMRI study. Neuroimage, 2006. 31(4): p. 1399-407.

94. Wong, K., et al., Functional brain interactions during reflexive micturition are absent from spinal cord injured rats with neurogenic bladder. Neurourology and Urodynamics, 2015. 34(5): p. 469-474.

95. Xiang, B., et al., Functional Brainstem Changes in Response to Bladder Function Alteration Elicited by Surgical Reduction in Bladder Capacity: A Functional Magnetic Resonance Imaging Study. Journal of Urology, 2010. 184(5): p. 2186-2191.

96. Nour, S., et al., Cerebral activation during micturition in normal men. Brain, 2000. 123: p. 781-789.

97. Blok, B.F.M., L.M. Sturms, and G. Holstege, Brain activation during micturition in women. Brain, 1998. 121: p. 2033-2042.

98. Blok, B.F., A.T. Willemsen, and G. Holstege, A PET study on brain control of micturition in humans. Brain, 1997. 120 ( Pt 1): p. 111-21.

99. Fukuyama, H., et al., Neural control of micturition in man examined with single photon emission computed tomography using (99)mTc-HMPAO. Neuroreport, 1996. 7(18): p. 3009-3012.

100. Friston, K.J., et al., Psychophysiological and modulatory interactions in neuroimaging. Neuroimage, 1997. 6(3): p. 218-29.

101. Kong, J., et al., Intrinsic functional connectivity of the periaqueductal gray, a resting fMRI study. Behavioural Brain Research, 2010. 211(2): p. 215-219.

102. Kleinhans, N.M., et al., Alterations in Connectivity on Functional Magnetic Resonance Imaging with Provocation of Lower Urinary Tract Symptoms: A MAPP Research Network Feasibility Study of Urological Chronic Pelvic Pain Syndromes. Journal of Urology, 2016. 195(3): p. 639-645.

103. Tadic, S.D., et al., Abnormal connections in the supraspinal bladder control network in women with urge urinary incontinence. Neuroimage, 2008. 39(4): p. 1647-1653.

104. Pantoni, L., Leukoaraiosis: from an ancient term to an actual marker of poor prognosis. Stroke, 2008. 39(5): p. 1401-3.

105. Kuchel, G.A., et al., Localization of Brain White Matter Hyperintensities and Urinary Incontinence in Community-Dwelling Older Adults. Journals of Gerontology Series aBiological Sciences and Medical Sciences, 2009. 64(8): p. 902-909.

106. Tadic, S.D., et al., Brain activity during bladder filling is related to white matter structural changes in older women with urinary incontinence. Neuroimage, 2010. 51(4): p. 12941302.

107. Pin-Wen Liao, Shih-Ping Liu, Hong-Jeng Yu, Yeong-Shiau Pu, Chen-Hsun Ho, Acute Urinary Retention after Brainstem Infarction. Incont Pelvic Floor Dysfunct, 2013(7): p. 10-11.

108. Cho, Y.S., et al., A novel intracerebral hemorrhage-induced rat model of neurogenic voiding dysfunction: Analysis of lower urinary tract function. Molecular Medicine Reports, 2015. 12(2): p. 2563-2569. 
109. Betts, C.D., R. Kapoor, and C.J. Fowler, Pontine Pathology and Voiding Dysfunction. British Journal of Urology, 1992. 70(1): p. 100-102.

110. Sakakibara, R., et al., Mechanism of bladder dysfunction in idiopathic normal pressure hydrocephalus. Neurourol Urodyn, 2008. 27(6): p. 507-10.

111. Cho, Y.S., et al., Oral mucosa stem cells alleviates spinal cord injury-induced neurogenic bladder symptoms in rats. Journal of Biomedical Science, 2014. 21.

112. Dasgupta, R., et al., Changes in brain activity following sacral neuromodulation for urinary retention. Journal of Urology, 2005. 174(6): p. 2268-2272.

113. Papadopoulou, A., et al., MRI characteristics of periaqueductal lesions in multiple sclerosis. Mult Scler Relat Disord, 2014. 3(4): p. 542-51.

114. Charil, A., et al., Statistical mapping analysis of lesion location and neurological disability in multiple sclerosis: application to 452 patient data sets. Neuroimage, 2003. 19(3): p. 53244.

115. Yaguchi, H., et al., A case of acute urinary retention caused by periaqueductal grey lesion. J Neurol Neurosurg Psychiatry, 2004. 75(8): p. 1202-3.

116. Collins, A.A.W.F., Lower Urinary Tract Symptoms Associated with Parkinson's Disease in SUNY UNDERGRADUATE RESEARCH CONFERENCE. 2015.

117. Kitta, T., et al., Role of the Anterior Cingulate Cortex in the Control of Micturition Reflex in a Rat Model of Parkinson's Disease. J Urol, 2016. 195(5): p. 1613-20.

118. Uchiyama T, S.R., Liu Z, Yamamoto T, Ito T, Awa Y, Yamaguchi C, Yamanishi T, Hattori T, mechanism of lower urinary tract dysfunction in parkinson's disease; participation in basal ganglia circuitry and sensory and autonomic/emotional nervous systems, in International Continence Society. cairo.

119. Phagu, N.P., Neural Control of Micturition in a Rat Model of Parkinson's Disease. Undergraduate Research Journal for the Human Sciences, 2015. 14.

120. Kitta, T., et al., Brain activation during detrusor overactivity in patients with Parkinson's disease: A positron emission tomography study. Journal of Urology, 2006. 175(3): p. 994998.

121. Crook, J.J. and T.A. Lovick, Changes in excitability of midbrain micturition control circuitry during alterations in sleep-like brain states in rats \&\#x2013; implications for nocturnal enuresis. Autonomic Neuroscience: Basic and Clinical. 192: p. 31-32.

122. Silberstein, S.D., Migraine symptoms: results of a survey of self-reported migraineurs. Headache, 1995. 35(7): p. 387-96.

123. Weiller, C., et al., Brain stem activation in spontaneous human migraine attacks. Nat Med, 1995. 1(7): p. 658-60.

124. Sakakibara, R., et al., Micturitional disturbance in Wernicke's encephalopathy. Neurourology and Urodynamics, 1997. 16(2): p. 111-115.

125. Griffiths, D., et al., Cerebral control of the bladder in normal and urge-incontinent women. Neuroimage, 2007. 37(1): p. 1-7.

126. Tadic, S.D., et al., Brain Activity Measured by Functional Magnetic Resonance Imaging is Related to Patient Reported Urgency Urinary Incontinence Severity. Journal of Urology, 2010. 183(1): p. 221-228.

127. Blok, B.F., et al., Different brain effects during chronic and acute sacral neuromodulation in urge incontinent patients with implanted neurostimulators. BJU Int, 2006. 98(6): p. 123843.

128. An, X., et al., Prefrontal cortical projections to longitudinal columns in the midbrain periaqueductal gray in macaque monkeys. J Comp Neurol, 1998. 401(4): p. 455-79. 
129. Krout, K.E. and A.D. Loewy, Periaqueductal gray matter projections to midline and intralaminar thalamic nuclei of the rat. J Comp Neurol, 2000. 424(1): p. 111-41.

130. Blok, B.F. and G. Holstege, The central nervous system control of micturition in cats and humans. Behav Brain Res, 1998. 92(2): p. 119-25.

131. Lüsebrink, F., et al., Data from: T1-weighted in vivo human whole brain MRI dataset with an ultrahigh isotropic resolution of $250 \mu \mathrm{m}$. 2017, Dryad Data Repository.

132. Lusebrink, F., et al., T1-weighted in vivo human whole brain MRI dataset with an ultrahigh isotropic resolution of 250 mum. Sci Data, 2017. 4: p. 170032.

133. Han, F., Y.F. Zhang, and Y.Q. Li, Fos expression in tyrosine hydroxylase-containing neurons in rat brainstem after visceral noxious stimulation: an immunohistochemical study. World J Gastroenterol, 2003. 9(5): p. 1045-50.

134. Meyer, P.J., et al., Contribution of dopamine receptors to periaqueductal gray-mediated antinociception. Psychopharmacology (Berl), 2009. 204(3): p. 531-40.

135. Clements, J.R., et al., Immunocytochemical localization of serotonin in the rat periaqueductal gray: a quantitative light and electron microscopic study. J Comp Neurol, 1985. 236(1): p. 60-70.

136. Pazos, A. and J.M. Palacios, Quantitative autoradiographic mapping of serotonin receptors in the rat brain. I. Serotonin-1 receptors. Brain Res, 1985. 346(2): p. 205-30.

137. Gundlach, A.L., Regional Subdivisions in the Midbrain Periaqueductal Gray of the Cat Revealed by In Vitro Receptor Autoradiography, in The Midbrain Periaqueductal Gray Matter: Functional, Anatomical, and Neurochemical Organization, A. Depaulis and R. Bandler, Editors. 1991, Springer US: Boston, MA. p. 449-464.

138. Williams, F.G. and A.J. Beitz, Ultrastructural morphometric analysis of GABAimmunoreactive terminals in the ventrocaudal periaqueductal grey: analysis of the relationship of GABA terminals and the GABAA receptor to periaqueductal grey-raphe magnus projection neurons. J Neurocytol, 1990. 19(5): p. 686-96.

139. Reichling, D.B. and A.I. Basbaum, Contribution of brainstem GABAergic circuitry to descending antinociceptive controls: I. GABA-immunoreactive projection neurons in the periaqueductal gray and nucleus raphe magnus. J Comp Neurol, 1990. 302(2): p. 370-7.

140. McCabe, R.T. and J.K. Wamsley, Autoradiographic localization of subcomponents of the macromolecular GABA receptor complex. Life Sci, 1986. 39(21): p. 1937-45.

141. Barbaresi, P., Cellular and subcellular localization of the GABA(B) receptor $1 a / b$ subunit in the rat periaqueductal gray matter. J Comp Neurol, 2007. 505(5): p. 478-92.

142. Stone, E., et al., GABAergic control of micturition within the periaqueductal grey matter of the male rat. Journal of Physiology-London, 2011. 589(8): p. 2065-2078.

143. Kwok, C.H.T., et al., Postnatal maturation of endogenous opioid systems within the periaqueductal grey and spinal dorsal horn of the rat. Pain, 2014. 155(1): p. 168-178.

144. Gutstein, H.B., et al., Mu and kappa opioid receptors in periaqueductal gray and rostral ventromedial medulla. Neuroreport, 1998. 9(8): p. 1777-1781. 


\section{Chapter 3}

A novel implantation technique for neuromodulation of the peripheral nervous system in rats

Aryo Zare, Gommert A. van Koeveringe, Yasin Temel, Ali Jahanshahi 


\begin{abstract}
:
Objectives: Neuromodulation of the peripheral nervous system (PNS) in freely moving rodents often requires extended wires to be monitored from a distant location. The route of access of the wires in terms of feasibility, animal's wellbeing and maintenance of the device settings merit consideration. Herein, we aimed to demonstrate a novel implantation technique for neuromodulation of the peripheral nervous system in rodents.
\end{abstract}

Materials and Methods: Inspired by our experience in electrode implantation for deep brain stimulation, we developed a novel implantation approach, which allows performing electrical modulation of the bladder. In order to prevent the wires being damaged by freely moving rats, we guided the wires subcutaneously toward the nape to reach a socket platform, which was eventually mounted on the skull.

Results: The surgical procedure was well tolerated by the operated animals. No complications such as peritoneal infection, gastrointestinal tract injury or skin inflammation are observed during or after surgery. The socket over the skull could stay in place for several months and allowed easy plugging/detachments throughout the bladder neuromodulation and behavioural testing in freely moving rats.

Conclusion: Subcutaneous tunnelling of the electrodes from the periphery and implanting the electrode on the skull is a feasible method in rats. This method is currently being used in our laboratory for PNS neuromodulation. This method can be used whenever manipulation of the peripheral organs is intended in freely moving rodents. This can encompass many procedures such as, neuromodulation, regional drug delivery, diagnostic monitoring of peripheral organs, electromyography, etc.

\title{
Key words:
}

Neuromodulation; bladder; subcutaneous tunneling; electrical implantation

\section{Introduction}

Neuromodulation involves implantation of stimulating (often together with recording) electrodes into specific parts of the nervous system. This is a growing therapeutic modality especially in conditions of insufficient response to pharmacotherapy and has evolved to be an effective therapy for patients with Parkinson's disease, essential tremor and dystonia. Following successful application in movement disorders, it's expanding in various neurological and psychiatric disorders [1,2]. Neuromodulation of the peripheral nervous system is rising in popularity as well. Sacral neuromodulation in particular has been used for various pelvic pathologies. It has been proven to be efficacious in dysfunction of urine storage (incontinence) and voiding [3], fecal incontinence [4] and 
female pelvic floor dysfunction [5]. For understanding underlying mechanisms, and both validation and safety studies, reliable animal models are required. Rodents have been the most commonly used laboratory animals. However continuous neuromodulation in freely moving rodents is challenging due to their natural tendency to damage devices extending from their body. Using a body harness with an attached backpack for mounting of devices is an option mainly used when the length of the wires is short and can be reached externally between the origin and target regions [6]. It also has the disadvantage of the discomfort of wearing a jacket for the animal, which may hinder especially behavioural experiments and also be the subject of wear and tear by the animal's bite. An alternative approach would be guiding the electrodes through the subcutaneous tissue toward a location not reachable by the animal. This is more comfortable for the animal and makes transfer of longer wires and tubes possible. Inspired by our experience in DBS studies, we suggest a surgical method that allows neuromodulation of the peripheral nervous system in freely moving rats. As the animals usually explore and manipulate implanted devices, we guided the wires subcutaneously towards the nape and mounted the plug board on the skull, which is virtually non-reachable by the rats.

\section{Material and methods}

Adult male Sprague-Dawley rats (body weight 300-350 g at the time of surgery) from Charles River were housed in the Central Animal Facility (Maastricht University, Netherlands). Rats were individually placed in Makrolon ${ }^{\mathrm{TM}}$ cages. Housing conditions for temperature $\left(21 \pm 1^{\circ} \mathrm{C}\right)$ and light (reversed $12 \mathrm{~h}$ light/dark cycle, 7 a.m. - 7 p.m. lights off, music on) were controlled and food and water were provided ad libitum. All experimental animal procedures were executed during the dark phase, under red light, when rodents are most active. For the surgical procedure, the induction (4\%) and maintenance $(1.5 \%)$ of anesthesia was achieved by isoflurane inhalation (IsoFlo $\AA$, Abbott Laboratories Ltd, Great Britain) and the temperature was monitored using a rectal thermometer and maintained within one degree of $37^{\circ} \mathrm{C}$ using a heating blanket. 


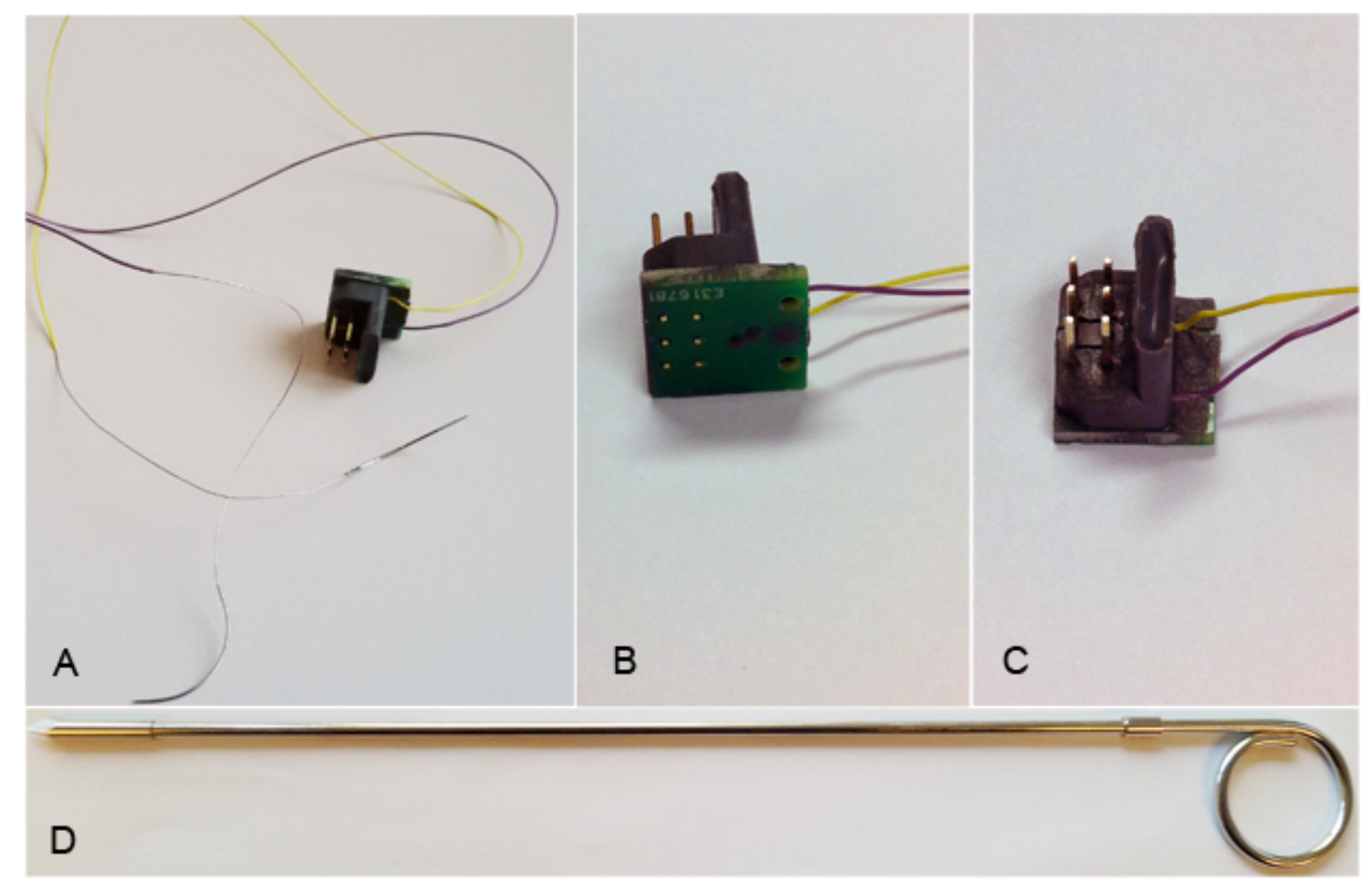

Figure 1. The custom made connector attached to pacemaker wires (A). This consists of a socket (B, C) capable of being connected to a pulse generator for provision of desired electrical parameters. A tunnelling trocar (D) serves as a subcutaneous dissector and transporter of the wires. The latest is used in human DBS neurosurgery.

In order to perform electrical stimulation of the urinary bladder in rats, a custom made construction was built (Figure 1). This consists of 2 pacing wires (StreamlineTM, Medtronic, France) implanted surgically in the detrusor muscle and a platform attached to the head used as a plug in order to connect the bipolar electrode to the stimulator. Notably, any other type of wire with biocompatible coating can be used, instead of these atrial wires. A midline incision was made from the orbital level to the occipital notch, which enabled adequate exposure of the skull. The rat was mounted on the stereotactic apparatus (Stoelting Co, Illinois, USA). The operation area was disinfected with chlorhexidine. A midline laparotomy was made to expose the urinary bladder. Blunt subcutaneous dissection was made through the abdominal incision toward the neck by a neurosurgical trocar (Figure 1-D) and then continued toward the cranial incision to make potential spaces (Figure 2). The wires were then tunnelled subcutaneously by the trocar, catching them from the scalp and guiding them toward the neck of the animal, and from there toward the abdomen (Figure 2). The pacing wires were driven into the detrusor muscle at the dome and the neck of the bladder and were sutured and secured (Figure 3). The bladder was then checked for any leakage before closing the abdomen. To anchor the plug of the construction over the skull, miniature screws were placed in the skull. The platform was positioned on top of the screws and fixed with dental cement (Paladur, 
Heraeus Kulzer GmbH, Germany) (Figure 2). Finally, the skin was carefully repositioned around the cemented construction. The neck incision was then sutured and closed.

A recovery period of two weeks was given before stimulation and behavioral testing. Freely moving neuromodulation experiment was performed to assess locomotor activity, anxiety and voiding pattern changes in rats during electrical stimulation of the urinary bladder, using the Open Field, Elevated Zero Maze and Voiding Behavior Tasks, respectively. Rats underwent the behavioral tests before and after surgery for electrode implantation under sham and stimulation conditions.
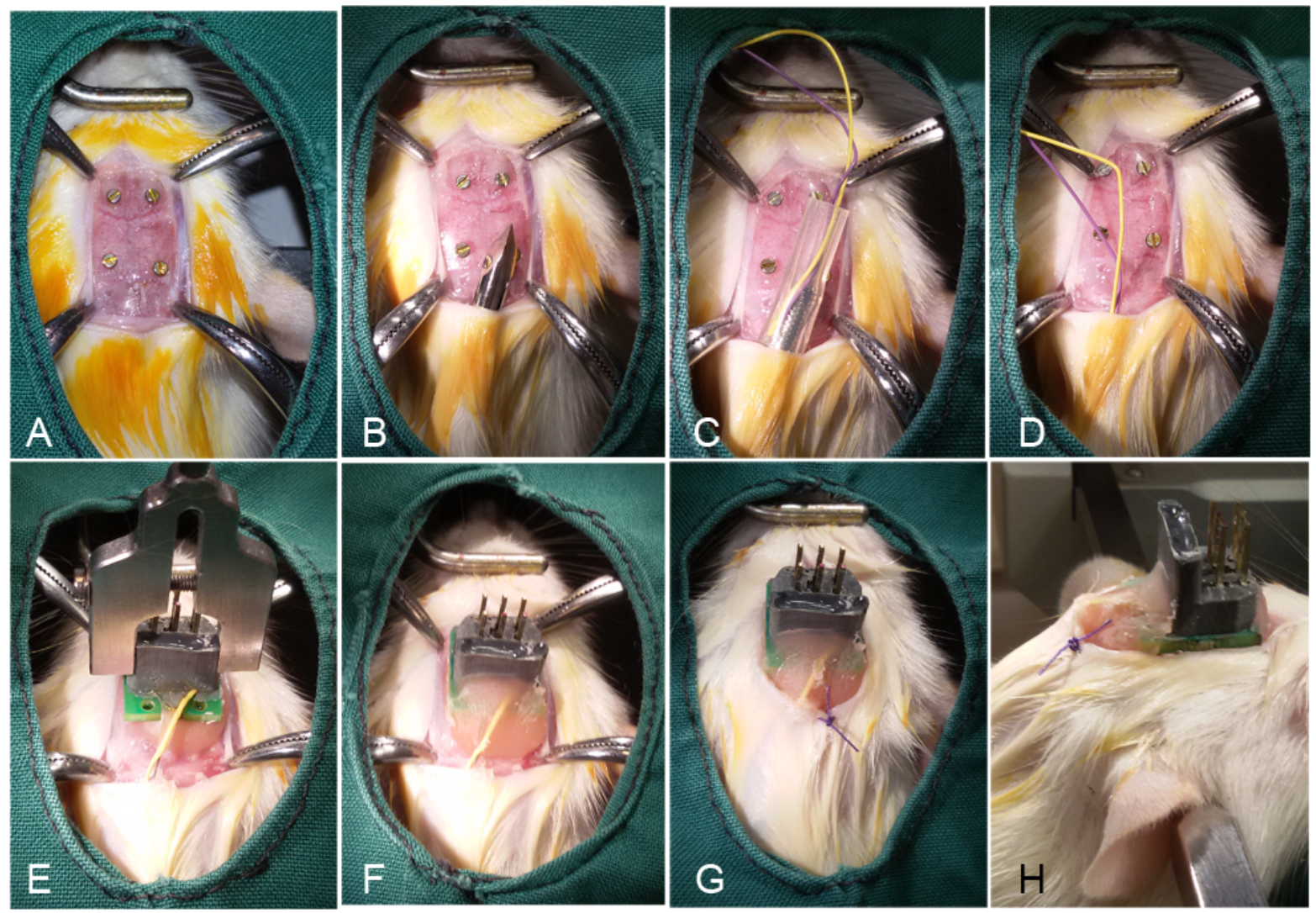

Figure 2. Subcutaneous guiding of the wires and implantation of the plug construction over the skull; a midline incision is made over the scalp to expose the skull. Four miniature screws are fixed onto the scull and serve as anchor (A). The trocar is passed subcutaneously from the abdominal incision toward the scalp (B) and catches the wires (C). The wires are then traversed through the potential space dissected by the trocar toward the abdominal location (D). The plug construction is laid over the skull (E) and fixed with dental cement (F), and the skin is sutured (G, H). The photographs are taken later throughout demonstration sessions and do not belong to an in vivo experiment. 

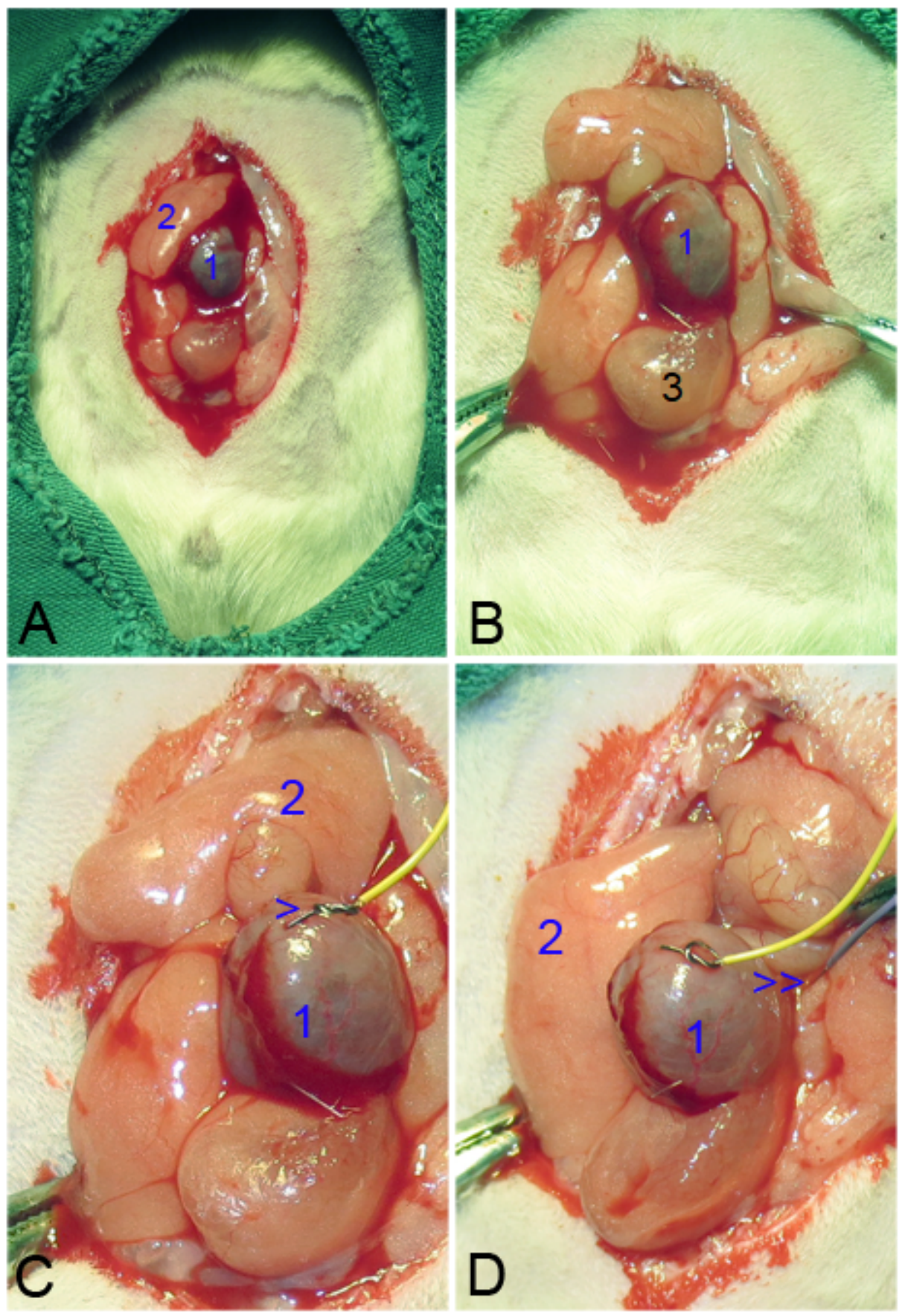

Figure 3. Implantation of pacemaker wires over the bladder; a suprapubic incision is made (A) and the bladder is exposed. Two pacemaker wires brought from the skull incision with the trocar and sutured to the detrusor muscle (C), one at the dome and one at the neck of the bladder ( $>$ and $>>$, respectively).1: bladder, 2: preputial gland, 3: prostate gland.

\section{Results}

Subcutaneous tunnelling of the electrodes from the periphery and implanting the electrode on the skull is a feasible method in rats. This surgical procedure is well tolerated by the operated animals. Complications such as peritoneal infection, gastrointestinal tract 
injury or skin inflammation during or after surgery are rare. The socket over the skull stays in place for several months and allows easy plugging/detachments throughout the bladder neuromodulation and behavioural testing in freely moving rats. This method is currently being practiced in our laboratory in PNS neuromodulation experiments. Using this method, we performed long term neuromodulation of the bladder in freely moving condition, in which not only micturition behaviour also possible motor, emotional and cognitive side effects were assessed. Moreover, neuroplastic changes in the micturition control circuits were evaluated. Electrophysiological, behavioral and histological finding of this study has been published.

\section{Discussion}

Mounting the equipment on a body harness and strapping it over the animal does not have the same quality as it is less comfortable for the animal and it still has the susceptibility to damage, by their manipulation. Working with freely moving animals with implanted materials demands the ease of access for the researcher together with the convenience for the animal. This assumes greater significance when the implantation occurs at abdominal location of a rodent, which is continuously in touch with the ground. To guide the terminal of the wires to a safe part of the rat body we took advantage of the natural characteristic of the rat skin. Rodents have a loose and easily retractable jacket. This feature makes scruffing and injection of large amounts of subcutaneous solutions possible and painless [7].

Subcutaneous implantation has been used in various ways both in human subjects and the experimental animals. Attempts have been made for extra-anatomic urinary diversion by subcutaneous tunnelling of the nephrostomy tube [8]. Similar subcutaneous urinary diversions were made by using silicone-PTFE prosthesis to bypass malignant or complex benign obstructions of the ureters that otherwise would necessitate permanent nephrostomy drainage [9]. Subcutaneous tunnelling of the ventriculostomy tube was also used in the neurosurgical field to decrease the rate of its infection [10]. Subcutaneous tunnelling of DBS extension wires is also performed by various methods in human subjects [11]. Aside from clinical applications, experimental animals were also subjected to subcutaneous tunnelling of the tubing's. The place of the concealment of the tube is usually at the back of the animal to halt their manipulation attempt. This technique was performed for the ventriculostomy tube in a Rhesus monkey model [12] or a central venous catheter in a rat model [13]. Subcutaneous placement of biocompatible insulated wires has been used for various reasons as in vagus nerve stimulation [14] and has been proven to be safe.

On the other hand the platform of plugs must have been mounted on somewhere both with minimal access of the animal and sufficient exposure for attachment to pulse generator at the time of the experiments. The surface of the skull is a stable plane, which has the capability of attachment of such platforms with miniature screws and dental cement. This has been shown to be well tolerated in rats [15]. This method can be used to perform investigations on internal organs of freely moving rats while remotely controlling the implanted apparatus on their skull. This can encompass neuromodulation, 
regional drug delivery for pharmacological investigations, diagnostic monitoring of peripheral vascular pressures or intravesical pressure and many other protocols. Such types of experiments are being performed in our group. There has been no evidence of infection or discomfort in the animals using this setting.

\section{Conclusion}

Subcutaneous tunnelling of the electrodes from the periphery toward the nape and thereafter implanting the connectors on the skull bone is a feasible and well-tolerated method in rats for experiments, which require stimulation or recording from peripheral nervous system or any form of monitoring of other organs.

\section{Acknowledgements}

The authors would like to thank the IDEE, Maastricht University for constructing the electrodes.

\section{Reference:}

1. Temel, Y. and A. Jahanshahi, Neuroscience. Treating brain disorders with neuromodulation. Science, 2015. 347(6229): p. 1418-9.

2. Temel, Y., et al., Neuromodulation in psychiatric disorders. Int Rev Neurobiol, 2012. 107: p. 283-314.

3. Sukhu, T., M.J. Kennelly, and R. Kurpad, Sacral neuromodulation in overactive bladder: a review and current perspectives. Res Rep Urol, 2016. 8: p. 193-199.

4. $\quad$ Rosen, A., et al., Effects of Sacral Neuromodulation on Urinary and Fecal Incontinence. Isr Med Assoc J, 2015. 17(6): p. 351-5.

5. Pettit, P.D., J.R. Thompson, and A.H. Chen, Sacral neuromodulation: new applications in the treatment offemale pelvic floor dysfunction. Curr Opin Obstet Gynecol, 2002. 14(5): p. 5215.

6. Ye, X., et al., A portable telemetry system for brain stimulation and neuronal activity recording in freely behaving small animals. J Neurosci Methods, 2008. 174(2): p. 186-93.

7. Rigalli, A. and V. Di Loreto, Substances Administration, in Experimental Surgical Models in the Laboratory Rat. 2016, CRC Press.

8. Paterson, P.J. and A. Forrester, Extra-anatomic urinary diversion. J Endourol, 1997. 11(6): p. 411-2.

9. Jabbour, M.E., et al., Percutaneous implantation of subcutaneous prosthetic ureters: longterm outcome. J Endourol, 2001. 15(6): p. 611-4.

10. Friedman, W.A. and J.K. Vries, Percutaneous tunnel ventriculostomy. Summary of 100 procedures. J Neurosurg, 1980. 53(5): p. 662-5.

11. Fontaine, D., et al., Two-step tunneling technique of deep brain stimulation extension wiresa description. Acta Neurochirurgica, 2013. 155(12): p. 2399-2402.

12. Mccully, C.L., et al., A Rhesus-Monkey Model for Continuous Infusion of Drugs into Cerebrospinal-Fluid. Laboratory Animal Science, 1990. 40(5): p. 520-525. 
13. Nett, J.E., K. Marchillo, and D.R. Andes, Modeling of Fungal Biofilms Using a Rat Central Vein Catheter, in Host-Fungus Interactions: Methods and Protocols, A.C. Brand and D.M. MacCallum, Editors. 2012, Humana Press: Totowa, NJ. p. 547-556.

14. Aalbers, M., et al., Animal models for vagus nerve stimulation in epilepsy. Exp Neurol, 2011. 230(2): p. 167-75.

15. Tan, S., et al., Experimental deep brain stimulation in animal models. Neurosurgery, 2010. 67(4): p. 1073-9; discussion1080. 


\section{Chapter 4}

\section{Neuronal activation in the periaqueductal gray matter upon electrical stimulation of the bladder}

Celine Meriaux, Ramona Hohnen\#, Sandra Schipper\#, Aryo Zare, Ali Jahanshahi, Lori A. Birder, Yasin Temel, Gommert A. van Koeveringe

\# Equal contribution

Frontiers in Cellular neuroscience

DOI: $10.3389 /$ fncel.2018.00133 


\begin{abstract}
Reflexes, that involve the spinobulbospinal pathway control both storage and voiding of urine. The periaqueductal gray matter (PAG), a pontine structure is part of the micturition pathway. Alteration in this pathway could lead to micturition disorders and urinary incontinence, such as the overactive bladder symptom complex (OABS). Although different therapeutic options exist for the management of OABS, these are either not effective in all patients.

Part of the pathology of OABS is faulty sensory signaling about the filling status of the urinary bladder, which results in aberrant efferent signaling leading to overt detrusor contractions and the sensation of urgency and frequent voiding. In order to identify novel targets for therapy (i.e. structures in the central nervous system) and explore novel treatment modalities such as neuromodulation, we aimed at investigating which areas in the central nervous system are functionally activated upon sensory afferent stimulation of the bladder. Hence, we designed a robust protocol with multiple readout parameters including immunohistological and behavioral parameters during electrical stimulation of the rat urinary bladder. Bladder stimulation induced by electrical stimulation, below the voiding threshold, influences neural activity in (1) the caudal ventrolateral PAG, close to the aqueduct, (2) the pontine micturition center and locus coeruleus, (3) the superficial layers of the dorsal horn, sacral parasympathetic nucleus and central canal region of the spinal cord. In stimulated animals, a higher voiding frequency was observed but was not accompanied by increase in anxiety level and locomotor deficits. Taken together, this work establishes a critical role for the vlPAG in the processing of sensory information from the urinary bladder and urges future studies to investigate the potential of neuromodulatory approaches for urological diseases.
\end{abstract}

Keywords: Periaqueductal gray, Bladder, Sensory, Brain-Bladder, Lower urinary tract symptoms 


\section{Introduction}

Overactive bladder symptom complex (OABS) is one of the most frequently encountered urological disorders. In the western world, the number of people suffering from OABS has been evaluated at almost 100 million [1-3]. The complex is defined as a medical condition encompassing urgency, increased daytime frequency, nocturia and urgency urinary incontinence (i.e., involuntary loss of urine associated with urgency) either isolated or in any combination [4]. Although this is not considered as a severe health condition, it has a significant impact on personal autonomy, self-esteem, quality of life, quality of sleep, and mental health and it creates an enormous economic burden $[2,5]$.

Nowadays, the first line treatment for OABS relies on the application of antimuscarinic drugs $[6,7]$. The second line therapies include botulinum toxin injection into the detrusor muscle [8-11], sacral neuromodulation [12-14] or invasive surgical interventions such as an augmentation cystoplasty [15]. However, these therapeutic interventions are only effective in a subset of patients and their effectiveness is limited in the remainder. One of the key elements of OABS is related to faulty sensory signaling of the filling status of the bladder. Knowledge about structures within the central nervous system involved in the micturition pathway can aid in developing novel rationally-designed treatment modalities and can help to identify patients, which are good candidates for specific lines of treatment.

The two functions of the lower urinary tract (LUT), i.e. urinary bladder and urethra, to store and periodically expel urine, are dependent upon central and local neural circuits located in the brain, the spinal cord and the peripheral ganglia [16-19]. Altered reflexes and sensory perceptions within the bladder - brain axis have emerged as a generally accepted model to explain pathologies such as OABS [20]. The modulation of sensory afferent functioning by means of neuromodulation could be considered a promising option for the treatment of OABS. However, in order to reach maximal efficacy and minimal side effects targets need to be identified.

One vital structure in the central pathway of the micturition pathway is the periaqueductal gray matter (PAG). The PAG receives ascending sensory fibers from the bladder, which pass through the dorsal horn of the spinal cord. The PAG is connected to the pontine micturition center (PMC; also called Barrington's nucleus). The PAG, in turn, is connected to other supraspinal structures of which some belong to the limbic system (such as the amygdala, insula) but also the prefrontal cortex and the thalamus [21-28].

It is generally believed that all ascending signals (such as mechanosensation [24] are relayed through the PAG to the PMC. The PMC is the site for the initiation of the micturition reflex [29-31]. Therefore the PAG is considered to be at the crossroads of ascending sensory information and inputs from higher centers that modulate these processes and plays the role of coordination center [32]. Inputs from higher cortical systems are related to emotions, i.e. anxiety, and interpretation of social aspects [33-35]. 
Based on neuroanatomical and cytoarchitectural features the PAG has been subdivided into four longitudinal columns spanning in the rostro-caudal direction, which are classified as the dorsomedial, dorsolateral, lateral and ventrolateral column [36-40]. Besides, their neuroanatomical distinction, these columns are also involved in different physiological processes. It has been described that the PAG is involved in autonomic (respiration and cardiovascular functions) as well as pain related functioning.

Several studies have investigated neuronal connections to and from the PAG by means of imaging or neuronal tracing. However, functional connectivity in response to afferent sensory stimuli and in particular stretch-related mechanoperception have -to our knowledge- never been investigated in a physiological manner.

Therefore, the present manuscript describes three groups of experiments. First, we identified an optimal stimulus applied to the bladder, in order to provoke sensory afferent stimulation in the absence of voiding. Second, we evaluated what behavioral consequences this stimulus has. We evaluated these consequences in the light of the different functions (i.e. anxiety and pain) that the PAG is mediating. Third, we investigated the pattern of neuronal activation in different parts of the central nervous system and in particular in the PAG. We did so by localizing and quantifying the amount of cFos positive cells in the various columns of the PAG, the PMC, the locus coeruleus, and several nuclei of the sacral spinal cord after urinary bladder stimulation in freely moving rats, with the previously optimized parameters. 


\section{Methods}

Subjects

Adult, male Sprague-Dawley rats (body weight 300-350 g at the time of surgery) from Charles River were housed in the Central Animal Facility (Maastricht University, Netherlands). Rats were individually placed in Makrolon ${ }^{\mathrm{TM}}$ cages. Housing conditions for temperature $\left(21 \pm 1^{\circ} \mathrm{C}\right.$ ) and light (reversed $12 \mathrm{~h}$ light/dark cycle, 7 a.m. - 7 p.m. lights off, music on) were controlled and food and water were provided ad libitum. All experimental animal procedures were executed during the dark phase, under red light, when rodents are most active.

\section{Bipolar stimulation electrode implantation}

For the purpose of these experiments we developed a novel methodology for the electrical stimulation of the detrusor muscle. The idea is derived from the principle of transurethral intravesical electrostimulation (IVES), which has been used to initiate nonvoiding contractions, which result in the depolarization of afferent sensory nerve fibers and subsequently a strong centrally-induced detrusor contraction [41-45].

We implanted rats chronically with a custom-made bipolar stimulation construct, which consists of two pacing wires (Streamline ${ }^{\mathrm{TM}}$, Medtronic, France) that were surgically implanted in the bladder wall. The wires were tunneled transdermally and a platform attached to the head was used as plug in order to easily connect an external power source.

For the surgical procedure, anesthesia was achieved by isoflurane inhalation (IsoFlo®, Abbott Laboratories Ltd, Great Britain; induction: 4\%, maintenance: $1.5 \%$ ) and the temperature was monitored using a rectal temperature probe and maintained within one degree of $37^{\circ} \mathrm{C}$ using a heating blanket. The abdomen, the neck and the head were shaved and disinfected using Betadine ${ }^{\circledR}$. A small incision was made in the neck of the animal to subcutaneously tunnel the wires. A midline laparotomy was made to expose the urinary bladder and insert the exposed pacing wires between the serosa and the muscular layer. Two configurations have been used for the implantation of the bipolar electrode: (i) One pacing wire at the level of the dome and the other one at the level of the neck of the bladder, close to the urethra (hereafter abbreviated as: D-N) (ii) Both pacing wires at the level of the neck of the bladder (hereafter abbreviated as: N-N). To keep the electrode in place, to insulate the tips and protect the surrounding tissues from scratching, pieces of polyethylene tubing were sealed at the free end of wires. The abdominal incision was then closed. After installing the rat in the stereotactic apparatus (Stoelting model 51950, Stoelting Co, Illinois, USA), a dermal incision and periostomy were performed to facilitate anchoring of the construct to the skull by using miniature screws (stainless steel, 1 x 2 $\mathrm{mm}$ ). The platform was positioned on top of them and fixed with dental cement (Paladur, Heraeus Kulzer GmbH, Germany). For cFos immunostaining and behavioral experiments, a recovery period of two weeks was given before stimulation and testing. 


\section{Electrical stimulation of the urinary bladder and in vivo cystometry}

To evaluate which parameters were optimal for electrical stimulation of the urinary bladder, an experiment was performed combining electrical stimulation and in vivo cystometry in five rats under terminal anesthesia with urethane $(1.5 \mathrm{~g} / \mathrm{kg})$. Besides the two electrodes, a catheter was implanted at the dome of the bladder in order to measure intravesical pressure. For this reason, electrodes were implanted at opposing sides in the lateral bladder wall for all animals in this experiment. The catheter was made of PE-50 tubing (BD Medical, USA) with a cuff and inserted into the bladder dome during the laparatomy through the low abdominal incision and held in place with a purse-string suture.

After the recovery period, the bladder catheter was connected via a T-tube to a pressure transducer and an infusion pump. Saline solution was infused into the bladder at a flow rate of $90 \mu \mathrm{l} / \mathrm{min}$. The bladder was filled with saline up to $0.6 \pm 0.2 \mathrm{ml}$ and the grade of bladder filling was kept stable during the stimulation protocol. The intravesical pressure was recorded continuously using a disposable IBP pressure transducer (DPT-6000, Codan pvb Medical GmbH, Germany) and the MP150 data acquisition system equipped with an amplifier module and acquired with the AcqKnowledge software (AcqKnowledge 4.2 version, BIOPAC system Inc., California, USA). Simultaneously, stimulation was applied by connecting animals to a stimulus isolator (DS100, WPI Europe, Germany) driven by a digital stimulator (DS8000, WPI Europe, Germany). After bladder stabilization, electrical stimulation was given with constant current biphasic square wave pulses of $0.5 \mathrm{~ms}$. Studies showed that sensory nerves were more effectively stimulated with a pulse width of $0.5 \mathrm{~ms}$ whereas motor fibers were preferentially activated at shorter pulse duration (0.05 - $0.4 \mathrm{~ms}$ ) [46, 47]. Additionally, longer pulse duration (>2 $\mathrm{ms}$ ) might cause tissue damage.

We aimed at establishing optimal stimulation frequency (ranging from 10 to $200 \mathrm{~Hz}$ ) and intensity (ranging from 2 to $3 \mathrm{~mA}$ ) in order to induce intravesical pressure augmentation. In order to prevent confounding, a randomized stimulation paradigm with different frequencies was applied with electrical stimulations lasting 30 seconds followed by a stimulation-off period of 60 seconds to allow intravesical pressure to return to baseline.

After the experiment rats were sacrificed by means of decapitation and no further postmortem analysis took place.

\section{Stimulation induced behavioural changes}

To assess locomotor activity, anxiety and voiding pattern changes in rats during electrical stimulation of the urinary bladder, the following behavioral tests were performed: Open Field (OF), Elevated Zero Maze (EZM) and Voiding Behavior Tasks. Five rats underwent all behavioral tests in a repeated design after a period of habituation to the handler and experimental environment at the following time points: at baseline prior to the surgical 
procedure (Control condition), two weeks after the electrode implantation in the presence (Stimulated condition) and absence (Sham condition) of electrical stimulation. For all behavioural experiments the electrodes were implanted in the D-N configuration.

At the end of the experiments rats were sacrificed and their tissue was not used for further post-mortem investigations due to the potential confounding influence of repeated stimulation.

\section{Open Field Task}

Spontaneous locomotor activity was measured in the OF, which consists of a large Plexiglas square arena $(100 \times 100 \mathrm{~cm})$ with $40 \mathrm{~cm}$ high transparent Plexiglas walls and a dark Plexiglas floor. The trials were recorded under low light conditions and the total distance moved was automatically assessed via a video camera connected to a video tracking system (Ethovision, Noldus, The Netherlands).

\section{Elevated Zero Maze Task}

Anxiety was evaluated in the Elevated Zero Maze (EZM), a black plastic circular runway (100 cm in diameter, $10 \mathrm{~cm}$ path width) placed $70 \mathrm{~cm}$ above the floor level and equally divided into two opposite open and two opposite enclosed parts with $50 \mathrm{~cm}$ high side walls. To prevent falls, a 7-mm high rim surrounds the open arms. Animals were placed into one of the open arms facing a closed part and allowed to explore the maze for five minutes. Time spent in the open parts was automatically quantified by means of the Ethovision setup under low light conditions through an infrared video camera connected to a video tracking system.

\section{Voiding Behavior}

Voiding behavior was assessed with a filter paper assay in combination with videoassisted tracing as previously described for mice [48]. We adapted the procedure to the body size of rats by changing the size of the cage and re-calculating a standard curve for estimating the relation between spot size and volume of urine voided.

All rats were assessed at the same time of the day to minimize the effect of the circadian cycle on micturition frequency and volume. Testing started 24 hours after a habituation period lasting for five hours in which the animal was placed into the cage in which the measurement took place.

Animals were placed on an elevated mesh floor, in a standard cage with its floor covered by filter paper. For one-hour, voiding behavior of freely moving rats was observed using a video camera placed below the cage. Thereafter, the number of spots, and the volume of voided urine were assessed as indicators for the voiding frequency, and the total of 
discharged urine. For this purpose, the filter paper was exposed to ultraviolet light and spot quantity and size were measured in ImageJ.

Neuronal activation in the central nervous system

\section{Experimental groups}

Rats were randomly assigned to one of the five experimental groups: (I) Control group without electrode implantation ( $n=6)$, (II) Sham D-N group ( $n=6)$, (III) Sham N-N group $(n=6)$, (IV) Stim D-N group ( $n=7$ ) and (V) Stim N-N group ( $n=7)$. Sham animals underwent the same surgical procedure with electrode implantation and were connected to the stimulator without stimulation being performed.

\section{CFos immunohistochemistry}

Following a two-week recovery period, one-hour continuous electrical stimulation of the bladder in freely moving rats was administrated using the previously optimized stimulation parameters.

For this purpose, rats were placed in custom-made cages that had equal dimensions as their home cages. These cages were modified in a way that the top of the cage allowed the connection of the head-mounted connector to the stimulator. Before the start of the stimulation rats were habituated for one hour.

Ninety minutes after the end of stimulation [49-52], rats were transcardiac perfused with Tyrode's buffer and with fixation solution containing 4\% paraformaldehyde, 15\% picric acid, $0.05 \%$ glutaraldehyde in $0.1 \mathrm{M}$ phosphate buffer (pH 7.6) [53]. Thirty-two brains and six spinal cords (Control $n=2$, Sham $D-N n=2$ and Stim $D-N n=2$ ) were removed rapidly and post-fixed in fresh fixative solution (Fixation solution without glutaraldehyde) for two hours at $4^{\circ} \mathrm{C}$ prior to overnight immersions in an ascending series of sucrose $110 \%$ and $20 \%$ sucrose in $0.1 \mathrm{M}$ phosphate buffer) at $4^{\circ} \mathrm{C}$. Finally, tissue was snap-frozen in solid carbon dioxide and stored at $-80^{\circ} \mathrm{C}$ until being sectioned coronally into $30 \mu \mathrm{m}$ serial slices using a cryostat (Leica, Germany). Brains sections were collected into cups to be processed immunohistochemically as free-floating sections whereas spinal cord sections were mounted on gelatin-coated glass slides. Serial sections were kept at $-80{ }^{\circ} \mathrm{C}$ before staining.

We applied two different ways of visualizing cFos. For the brain sections, we used the indirect immunohistochemical (3,3'-Diaminobenzidine (DAB)) staining method employing amplification by means of streptavidin and nickel-diaminobenzidine chromogen enhancement according to previous protocols [54] whereas for spinal cord sections we chose fluorescent visualization. Because brain sections are known for possessing auto-fluorescence after formaldehyde fixation [55-57] and we expected low 
cFos epitope expression, we opted for the indirect immunohistochemical visualization in the brain. In the spinal cord, the secondary aim was to perform double labeling techniques, which is facilitated by the use of fluorophores.

Brain sections containing the PAG (Bregma AP: -6.3 to $-8.3 \mathrm{~mm}$ ) and the pons (Bregma: AP: -9.6 to $10.0 \mathrm{~mm}$ ) were incubated overnight at $4{ }^{\circ} \mathrm{C}$ with monoclonal mouse anti-cFos primary antibody (1:2,000 dilution, Santa Cruz Biotechnology Inc., USA), washed with Tris-buffered saline (TBS) and TBS-Triton X-100, and then incubated with the biotinylated donkey anti-mouse secondary antibody (1:400 dilution, Jackson Immunoresearch Laboratories, USA) for one hour. This was followed by exposure to avidin-biotin-peroxidase complex (1:800, Elite ABC-kit, Vestastatin, Vector Laboratories, USA) for two hours. The staining was visualized using a 3,3-diaminobenzidine (DAB) solution and nickel intensification. Brain sections were mounted on gelatin-coated glass slides, dehydrated and cover-slipped with Pertex mounting medium (Histolab Products AB, Sweden). Spinal cord sections (L6 to S2 segments) were incubated overnight at room temperature with monoclonal mouse anti-cFos primary antibody (1:2,000 dilution, Santa Cruz Biotechnology Inc, USA) and rinsed following the same protocol as for brain sections. Subsequently, a two-hour incubation with the secondary antibody Alexa Fluor ${ }^{\circledR} 488$ conjugated donkey anti-mouse (1:100 dilution, Thermo Fisher Scientific, NY, USA) was followed by three washing steps with TBS and by coverslipping in $80 \%$ glycerol/TBS).

Photographs of the stained brain sections were taken (4x and 10x magnification) using a U-CMAD-2 digital camera mounted on an Olympus AX70 bright-field microscope (analySIS, Imaging System, Germany) and evaluated with ImageJ analysis software (ImageJ software, NIH, USA). We assessed results for the caudal and rostral regions of the PAG separately. In the rostral part of the PAG no ventrolateral (vl) column can be found and therefore results are based on the dorsomedial $(\mathrm{dm})$, dorsolateral $(\mathrm{dl})$ and lateral column of the PAG. In the caudal portion of the PAG all four columns are present [58] (Fig 1 Suppl Data). Columns were delineated from similar Bregma levels of two rostral (AP: $6.7 \mathrm{~mm}$ and $-7.0 \mathrm{~mm}$ ) and two caudal (AP: $-7.7 \mathrm{~mm}$ and $-8.0 \mathrm{~mm}$ ) PAG images per rat. The $\mathrm{LC}$ and $\mathrm{PMC}$ were delineated on the two hemispheres from one pontine region image per rat.

We assessed the quantity of cFos positive cells in the brain in the following way. First, we acquired pictures from the region of interest with a low magnification (4x). Second, we delineated the region or columnar areas with a higher magnification (10x), which resulted in an area, which was used for the quantification of the cell number (Fig 2 Suppl Data). In all cases, the delineation was based on standard anatomical hallmarks for the PAG (i.e. an angle of the circumference) or corresponding histological stainings of the consecutive section (Nissl staining for the PMC and LC) and the anatomical descriptions of these regions according to the standard rat brain atlas of Paxinos and Watson. Consequently, a thresholding method was applied in the delineated sections, which identified all cells, which had an intensity that exceeded the background [59]. Cells were then manually counted by two independent observers. Observers were blinded for 
treatment groups. The values were expressed as cells per area to correct for potential differences in the delineation of the areas and expressed as cells $/ \mathrm{mm}^{2}$.

A different workstation was used for the acquisition of pictures for the activation of cFos positive cells in the segments L6-S2 of the spinal cord. The analysis was performed online using a stereology workstation, i.e. a modified Olympus BX50 fluorescence microscope controlled by the Stereo Investigator software (MBF Bioscience, Vermont, USA). Delineations of the regions of interest (Laminae I to III of the dorsal horn, Medial gray region with the DCM, Lateral gray subdivision including the SPN and the ventral horn) were made on the microscopic images displayed on a monitor using 4x objective and adjusted at a higher magnification (10x). Within the delineated areas, the total number of cFos positive cells was evaluated using a 40x objective and the optical fractionator of the Stereo Investigator software by manually counting cFos positive cells. Because of the limited number of sections per region of interest, we did not extrapolate the data into a $2 \mathrm{D}$ volume but chose to present results per slice.

Electrode localization

The location of the electrode was assessed in a sample of the implanted animals by performing a macroscopic post-mortem examination. This was accompanied by the microscopic assessment of cryosection of the bladder. For this purpose, bladder strips were prepared, which were cut and rapidly assessed under bright field illumination. No staining procedures or quantifications were performed. This method revealed no overt signs of fibrosis, dis-location or inflammation in the assessed bladders.

\section{Statistics}

Data were presented as mean \pm S.E.M. values. For the evaluation of stimulation parameters, a one-way ANOVA was employed, followed by a Fisher PLSD post-hoc test. Histological and behavioral data were analyzed statistically with inter-group comparisons using $t$-test. All statistical analyses were performed with Sigma Plot 11.0 version for Windows (Systat Software). P-values lower than 0.05 were considered statistically significant.

Ethical approval

All procedures were in line with local and national guidelines and laws on animal welfare. All experimental protocols were ethically reviewed and approved by the Animal Experiments and Ethics Committee of Maastricht University. 


\section{Results}

Cystometric investigation for electrical stimulation parameter optimization The effect of different combinations of parameters (i.e. frequency and intensity) on the induced non-voiding detrusor contraction was evaluated to find the most effective setting for one-hour continuous electrical stimulation of the bladder with the bipolar electrode. Pressure responses were observed during electrical stimulation when the current intensity was higher than $2 \mathrm{~mA}$, for a frequency range of $20 \mathrm{~Hz}$ to $200 \mathrm{~Hz}$. For $10 \mathrm{~Hz}$ stimulations, an intensity of $3 \mathrm{~mA}$ was required to trigger an intravesical pressure increase. In all animals, the pressure increase, and the stimulus amplitude were positively correlated (Fig 1A). In five animals, the maximal pressure response $\left(4.55 \mathrm{cmH}_{2} \mathrm{O} \pm 1.07\right)$ was obtained with a current intensity of $3 \mathrm{~mA}$. The response to electrical stimulation of the bladder with a current intensity of $2 \mathrm{~mA}$ was characterized by a rise in pressure to reach a plateau and then a decrease towards the baseline (Fig 1A). Higher current intensities (2.5 mA and $3 \mathrm{~mA}$ ) elicited an acute increase of pressure, showed as a sharp peak, and followed by a decrease of pressure to the plateau level (Fig 1A). However, in order to avoid tissue injury and at the same time to avoid inducing a micturition contraction during long-term electrical stimulation, the current intensity of $2 \mathrm{~mA}$, the lowest current intensity capable of inducing a response, was chosen. Because the intravesical pressure responses could depend on the position of the electrode, we standardize the statistical analysis, by normalizing the data and expressed these as percentage of the maximal intravesical pressure response per animal. With the current intensity set at $2 \mathrm{~mA}$, the intravesical pressure responses obtained upon electrical stimulation were frequency-dependent (One-way ANOVA, $F(4,24)=61.175$, $p<0.001$ ) (Fig 1B). With a frequency of $10 \mathrm{~Hz}$, low intravesical pressure responses were induced $(6.08 \%$ $\pm 1.28)$. At $20 \mathrm{~Hz}$, the response was maximal $(54.4 \% \pm 5.2)$ and was not significantly different from the responses found using higher frequencies (NS, 20 vs. $50 \mathrm{~Hz}$ p=0.396; 20 vs. $100 \mathrm{~Hz}$ p=0.963; 20 vs. $200 \mathrm{~Hz}$ p=0.175, Fischer PLSD). The observed response to electrical stimulation with $200 \mathrm{~Hz}$ was lower than with the intermediate frequencies (50 $\mathrm{Hz}$ vs. $200 \mathrm{~Hz}, 56.7 \% \pm 4.4$ vs. $48.04 \% \pm 2.2$, p=0.034). To minimize tissue damage due to continuous electrical stimulation, we opted for the frequency of $20 \mathrm{~Hz}$, the lowest frequency stimulation giving a maximal pressure response. Therefore, the optimal combination of both parameters inducing an adequate sensation inducing contraction of the bladder following electrical stimulation was determined as having a frequency of 20 $\mathrm{Hz}$ and an amplitude of $2 \mathrm{~mA}$ and has been used for the subsequent set of experiments. 
A

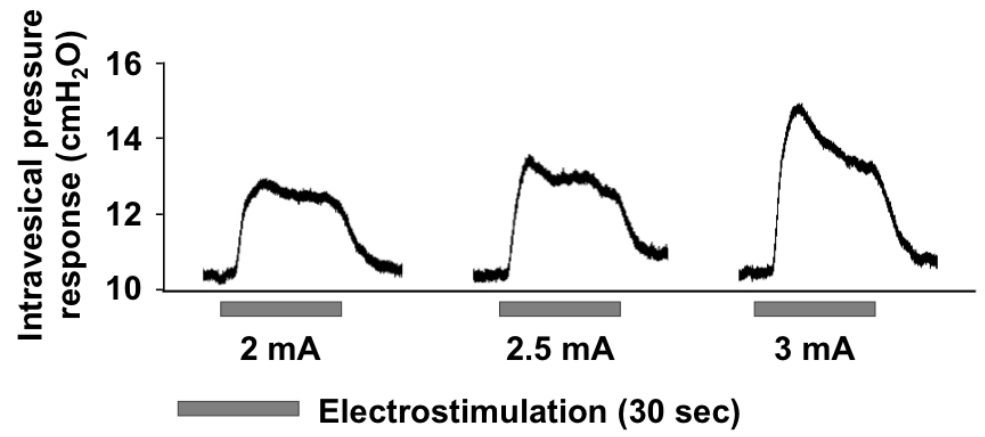

B

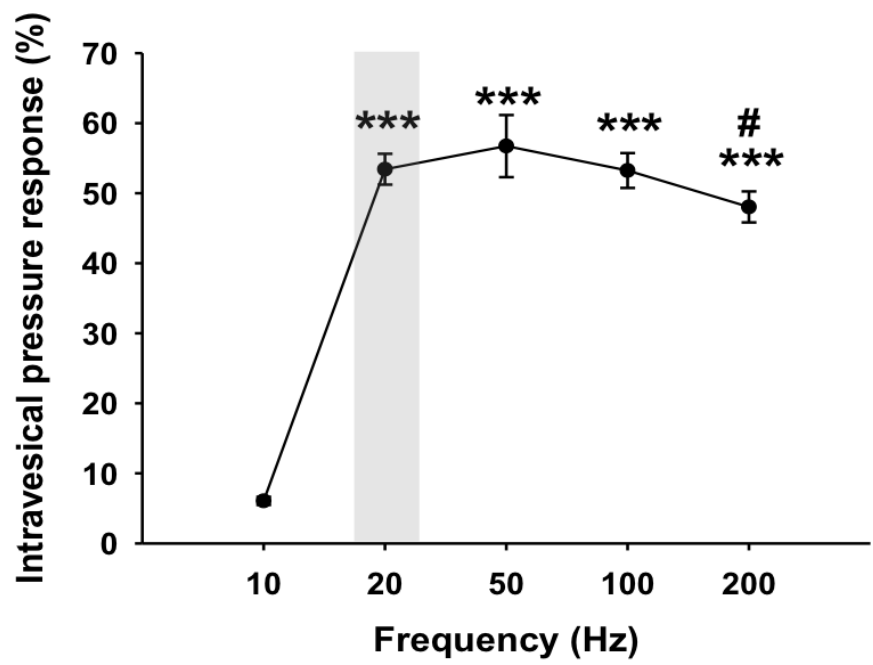

Figure 1. Intravesical pressure responses during electrical stimulation of the urinary bladder. (A) Representative intravesical pressure responses during electrical stimulation with a frequency adjusted to $20 \mathrm{~Hz}$ and an intensity set at 2, 2.5 and $3 \mathrm{~mA}$. (B) Intravesical pressure responses during electrical stimulation with intensity of $2 \mathrm{~mA}$ and variable frequency from $10 \mathrm{~Hz}$ and $200 \mathrm{~Hz}$. The intravesical pressure responses were significantly lower for $10 \mathrm{~Hz}$ frequency compared to frequencies higher than $20 \mathrm{~Hz}$. The increase in intravesical pressure following a stimulation with $200 \mathrm{~Hz}$ was lower than with $50 \mathrm{~Hz}$. Values are represented as means \pm S.E.M. $n=5$. One-way ANOVA, $\mathbf{F}(4,24)=61.175$, ${ }^{* * *}$ vs. $10 \mathrm{~Hz}, \mathrm{p}<0.001$; \# vs. $50 \mathrm{~Hz}, \mathrm{p}=0.034$

Behavioral measurements during electrical stimulation of the urinary bladder After the electrode implantation, in both stimulated and non-stimulated animals, characteristic behavioral signs of visceral pain were assessed. No behavioral modifications including lacrimation, piloerection, tail hyperextension, abdominal contraction, arched posture, lower abdomen licking, and backward withdrawal movements were displayed in neither the sham nor the stimulated conditions.

Previous works suggested that stress and pain are related to the activation of specific columns in the PAG [36, 37]. For instance, the dorsal PAG is linked to avoidance behavior, the lateral PAG is correlated to fight and flight whereas the ventrolateral PAG is 
associated with freezing and immobility. To confirm that the neuronal activation observed in the PAG related to bladder-related functioning and was not triggered by confounding behaviors potentially generated by electrode implantation or electrical stimulation of the urinary bladder, the locomotor behavior was evaluated in the open field. Analysis of the behavioral data in the open field showed that the total distance traveled was not significantly different in stimulated animals compared to sham animals (Sham vs. Stim, $5063.8 \mathrm{~cm} \pm 135.9$ vs. $5271.3 \mathrm{~cm} \pm 559.2$, $\mathrm{p}=0.753$ ). Therefore, neither the implantation of the bipolar electrode nor the electrical stimulation of the bladder alters spontaneous locomotor activity.

Moreover, animals were tested in the EZM to determine whether anxiety level was affected by the experimental procedure. No significant effect was found between the control and sham conditions ( $t$-test, NS, $\mathrm{p}>0.05$ ). Moreover, the time spent in open arms of the EZM was comparable between the sham and stimulated conditions (Sham vs. Stim, $95.7 \pm 34.8$ vs. $106.1 \pm 18.6, \mathrm{p}=0.805$ ) indicating no change in terms of anxiety-like behavior.

Also, we assessed whether stimulation changed voiding behavior (Fig 2A). The quantification of voiding spots reveals no following electrode implantation (Control vs. Sham, $4.7 \pm 0.3$ vs. $6.0 \pm 0.6, p=0.116$; Fig $2 \mathrm{~B}$ ). However, electrical stimulation of the bladder caused a significant increase of voiding frequency compared to the un-stimulated condition (Sham vs. Stim, $6.0 \pm 0.6$ vs. $11.0 \pm 1$, p = 0.012).

We estimated the total volume of voided urine during the VB by means of a volumetric calibration, which revealed a linear relation between spot size and volume voided. Video recordings were used to discriminate between overlapping urine spots. We found that the total volume of voided urine was comparable before and after surgery (Control vs. Sham, $0.61 \mathrm{ml} \pm 0.08$ vs. $0.72 \mathrm{ml} \pm 0.07$, $\mathrm{p}=0.33$; Fig $2 \mathrm{C}$ ). Moreover, the total volume of voided urine was not altered in stimulated rats (Sham vs. Stim, $0.72 \mathrm{ml} \pm 0.07$ vs. $0.78 \mathrm{ml}$ $\pm 0.12, \mathrm{p}=0.652$ ). The duration of the test we used resulted in a few voids at baseline. Therefore, we could not reliably quantify whether changes in the voiding pattern occurred in the different experimental conditions.

In summary our behavioral results indicate that there were no factors such as anxiety or pain, that could explain the activation of PAG columns. However, stimulation induced a higher frequency of voiding with smaller volumes per void. 
A

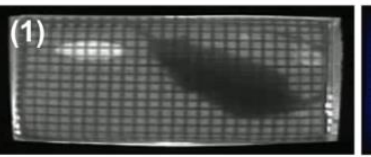

(2)

B

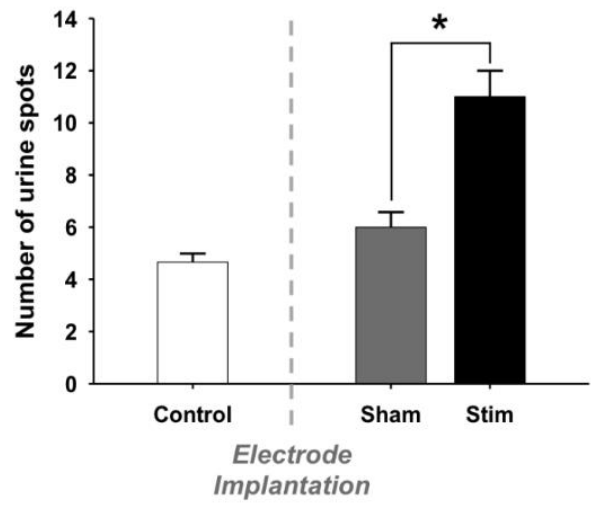

(3)

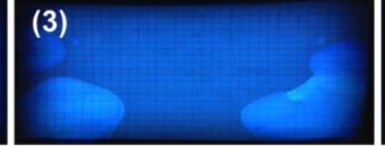

(4)

C

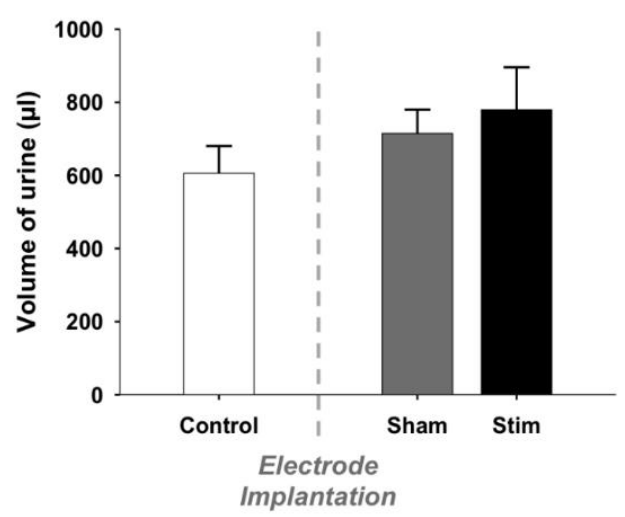

Figure 2. Voiding behavior: Voiding parameters. (A) Representative photographs of (1) an extract from the video recorded during a voiding behavior task (VBT) and filter papers from the same rat (2) before the surgery (Control), (3) after the electrode implantation (Sham) and (4) during electrical stimulation of the bladder (Stim). (B) Number of urine spots. During the stimulation, rats voided more frequently than when the stimulation was switched-off. Values are represented as means \pm S.E.M. Control $n=5$, Sham $n=5$ and Stim $\mathrm{n}=5$. $t$-test, Sham vs. Stim, ${ }^{*} \mathbf{p}<0.05$. (C) Total volume of voided urine. The total volume of urine spots was not significantly larger in stimulated condition compared to sham and control conditions

Effect of electrical stimulation of the urinary bladder on cFos immunoreactivity in the PAG

The cFos expression and its topographical localization in the PAG following electrical stimulation of the bladder were investigated to detect induced neuronal activation. Sham groups were used to test whether the presence of the electrode or the procedure of the electrode implantation per se were able to induce cFos expression in the PAG. In all sham groups, the cFos expression did not differ from control animals (Control vs. Sham, t-test, NS, p>0.05; Figs 3 and 4). No effect of electrode implantation (control vs. sham), regardless of pacing wires configuration, i.e. N-N vs. D-N, was observed on neuronal activation in any column of the PAG. Electrical stimulation of the urinary bladder upregulated cFos expression in several PAG columns compared to sham animals. This effect will be discussed separately for the rostral and caudal part of the PAG below.

Activation of neurons in the rostral part of the PAG

In both electrode configurations there was a significant increase of cFos positive cells in all columns of rostral part of the PAG except for a non-significant difference between sham and stimulated animals in the N-N configuration in the dl PAG. For N-N stimulated animals, the number of cFos positive cells in the IPAG and dmPAG was higher compared 
to sham control animals (Sham vs. Stim, IPAG and dmPAG, ${ }^{*} p=0.044$ and ${ }^{*} p=0.046$, respectively; Figs $3 \mathrm{~A}-\mathrm{C}$ ). Electrical stimulation of the urinary bladder in $\mathrm{D}-\mathrm{N}$ group increased the number of activated neurons in the IPAG, dIPAG and dmPAG (Sham vs. Stim, IPAG, dmPAG and dmPAG, ${ }^{* * *} \mathrm{p}<0.001,{ }^{*} \mathrm{p}=0.044$ and ${ }^{* * *} \mathrm{p}=0.001$, respectively; Figs $3 \mathrm{D}$ F).

A

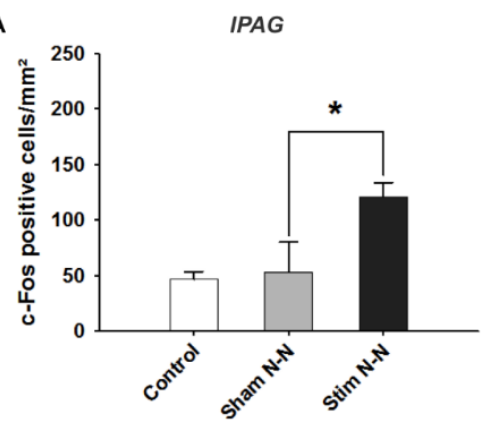

D

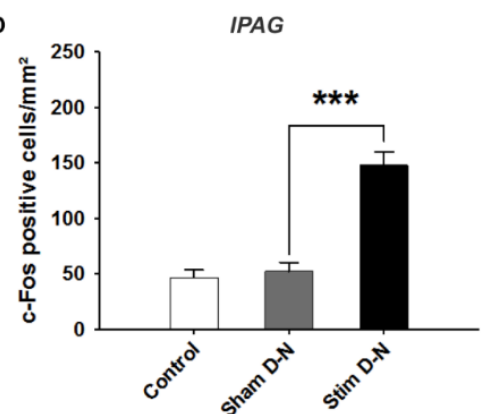

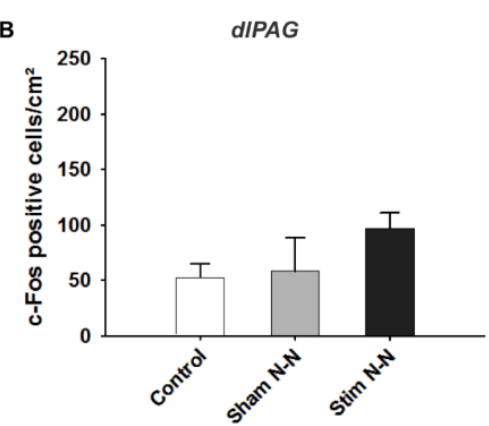

E

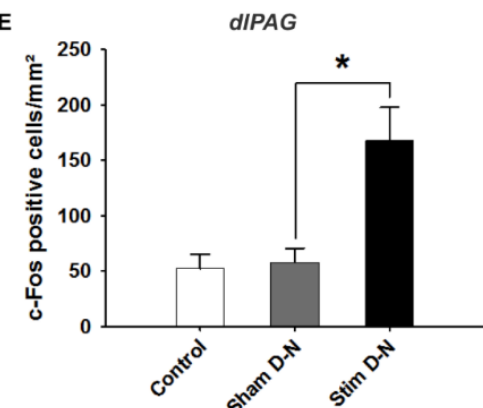

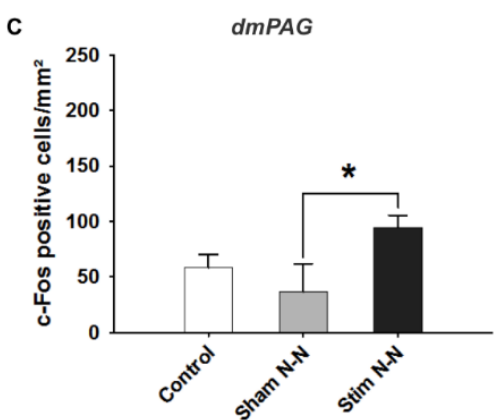

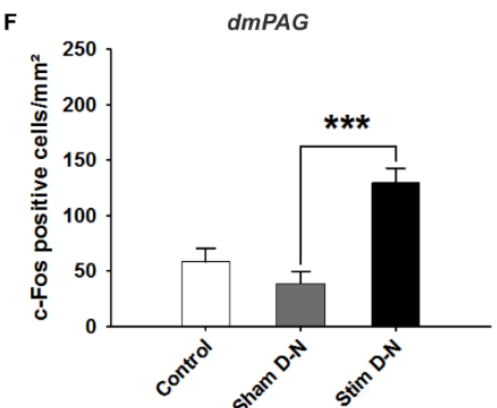

Figure 3. Expression of cFos immunoreactive cells in the rostral PAG. Cumulative data showing the means and S.E.M. of cFos expression for N-N group in the rostral (A) IPAG, (B) dIPAG, (C) dmPAG and for D-N group in the rostral (D) IPAG, (E) dIPAG, (F) dmPAG. Control $n=12$, Sham N-N n = 12 Sham D-N n = 12, Stim N-N n = 14 and Stim D-N n = 14. t-test, ${ }^{*}$ < $<0.05$ and ${ }^{* * *} \mathrm{p}<0.001$

Activation of neurons in the caudal part of the PAG

For N-N electrode configuration, no statistically significant difference in the number of cFos positive neurons was found in the caudal PAG between stimulated and sham groups (t-test, NS, p>0.05; Figs 4A-D). For D-N electrode implantation, cFos counts in the ventrolateral subdivision of the caudal PAG were significantly greater in stimulated compared to sham rats (Sham vs. Stim, $48.8 \pm 11.9$ vs. $177.4 \pm 23.1$ cells $/ \mathrm{mm} 2 ; \mathrm{p}=0.003$; Fig 4E). This was also the case for lateral and dorsomedial subdivisions of the caudal PAG (Sham vs. Stim, IPAG and dmPAG, ${ }^{*} \mathrm{p}=0.012$ and ${ }^{*} \mathrm{p}=0.024$, respectively; Figs $4 \mathrm{~F}$ and $4 \mathrm{H}$ ). The strongest effect size was seen in the caudal vlPAG compared to the other columns of the PAG (Fig 4). Moreover, the ventrolateral aspects of the caudal PAG close to the aqueduct in stimulated compared to sham $\mathrm{D}-\mathrm{N}$ rats seemed to be more densely populated 
with activated neurons (Fig 5). Indeed, this observation was quantitatively confirmed by cFos cells counting, showing that the majority $(53.8 \% \pm 7.8 \%)$ of activated neurons were located in the central one third closest to the aqueduct of the vlPAG area. We did not observe the clustering of neurons in other columns.
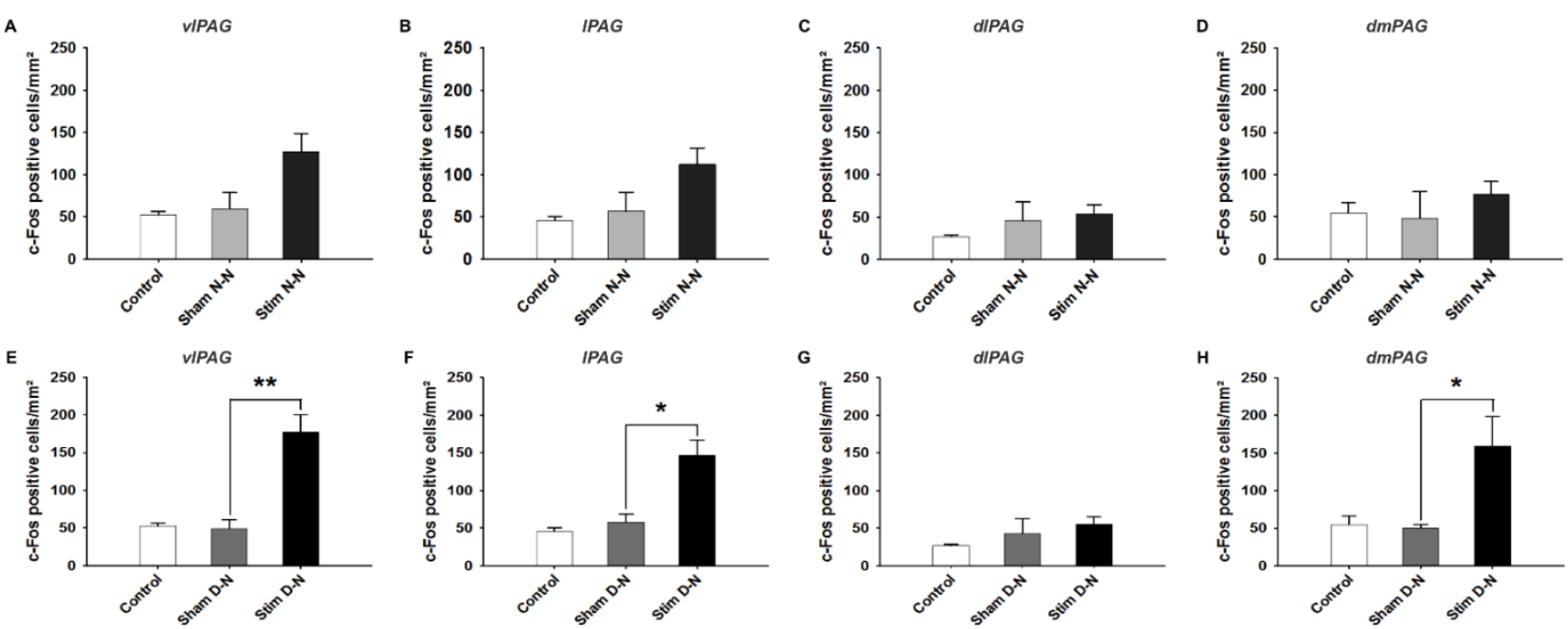

Figure 4. Expression of cFos immunoreactive cells in the caudal PAG. Cumulative data showing the means and S.E.M. of cFos expression for $\mathrm{N}-\mathrm{N}$ animals in the caudal (A) vlPAG, (B) IPAG, (C) dIPAG, (D) dmPAG and for D-N animals in the caudal (E) vlPAG, (F) IPAG, (G) dIPAG, (H) dmPAG. Control $n=12$, Sham $N-N n=12$ Sham D-N $n=12$, Stim N-N $n=14$ and Stim D-N n $=14$. t-test, ${ }^{*}$ p $<0.05$ and ${ }^{* *}$ p $<0.005$

A

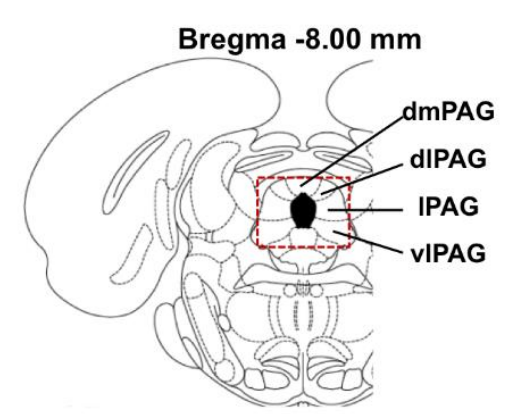

B

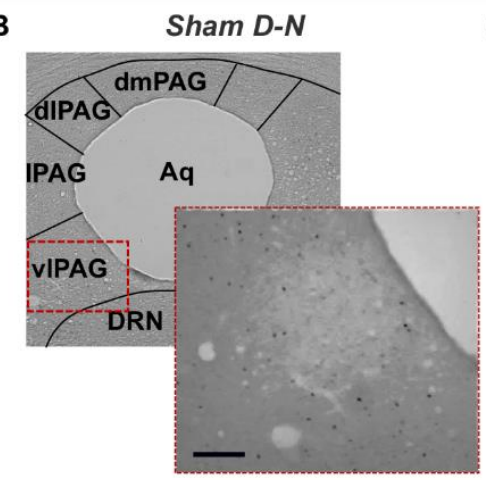

C

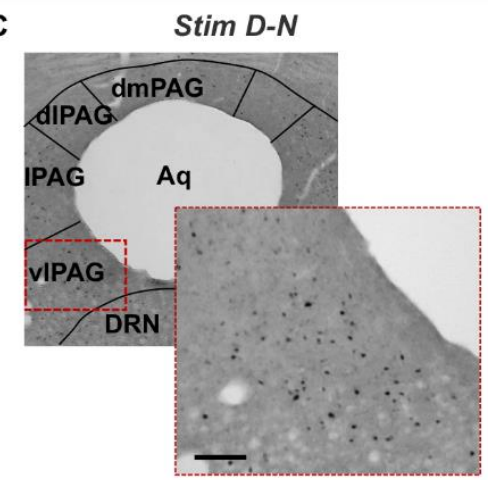

Figure 5. Distribution of cFos immunoreactive cells in the caudal PAG. (A) Coronal diagram of the rat brain at corresponding Bregma level AP $\mathbf{- 8 . 0 0 ~} \mathbf{m m}$ (Rat brain atlas, Paxinos \& Watson, [60]). Representative low power photomicrographs of coronal brain sections stained for cFos showing the PAG of (B) non-stimulated (Sham) rat and (C) stimulated rat (Stim) with bipolar stimulation electrode implanted in the bladder wall at the dome and neck levels. The higher power photomicrographs in the lower right corners focus on the central region of the vIPAG column. Aq: aqueduct, DRN: dorsal raphe nucleus, dIPAG: dorsolateral PAG, dmPAG: dorsomedial PAG, IPAG: lateral PAG and vlPAG: ventrolateral PAG. Scale bar $=100 \mu \mathrm{m}$ 
Effect of electrical stimulation of the urinary bladder on cFos immunoreactivity in the pons

The changes in cFos expression in the PMC and the locus coeruleus (LC) were examined following electrical stimulation of the urinary bladder for the D-N group only, because this configuration showed the biggest effect size for the PAG. The numbers of cFos positive cells for both pontine structures was similar in control and sham animals (Control vs. Sham, t-test, NS, p>0.05) (Figs 7A and 7B). By contrast, electrical stimulation of the urinary bladder significantly increased the number of activated neurons in the PMC and LC (Sham vs. Stim, PMC and LC, ${ }^{* * *} \mathrm{p}<0.001,{ }^{* *} \mathrm{p}=0.004$, respectively; Figs 6 and 7). Interestingly, whereas the activated neurons were distributed homogeneously throughout PMC, the cFos labeled neurons were mainly located in the dorsal part of the LC (Fig 6C).

A

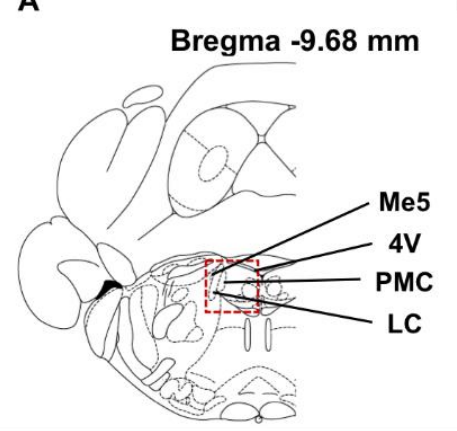

B

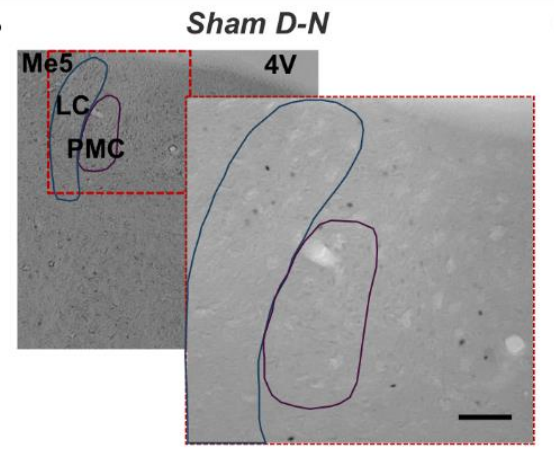

C

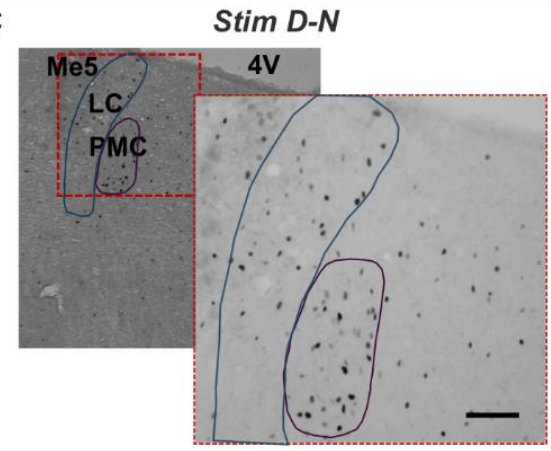

Figure 6. Distribution of cFos immunoreactive cells in the pontine structures. (A) Coronal diagram of the rat brain at corresponding Bregma level AP $\mathbf{- 9 . 6 8} \mathbf{~ m m}$ (Rat brain atlas, Paxinos \& Watson, [60]). Representative low power photomicrographs of coronal brain sections stained (B) with Nissl staining and for cFos showing the dorsal pons of (C) nonstimulated (Sham) rat and (D) stimulated rat (Stim) with bipolar stimulation electrode implanted in the bladder wall at the dome and neck levels. The higher power photomicrographs in the lower right corners show the PMC and locus coeruleus. 4V: fourth ventricle, PMC: Pontine micturition center or Barrington's nucleus, LC: locus coeruleus, Me5: mesencephalic trigeminal tract. Scale bar $=150 \mu \mathrm{m}$ 
A

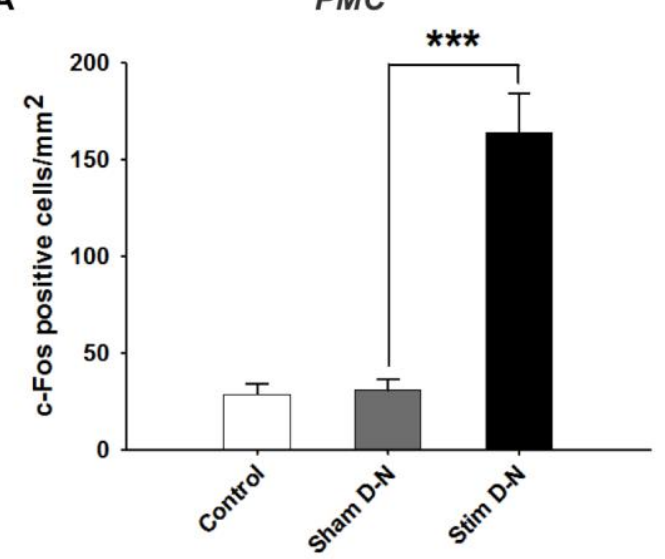

B

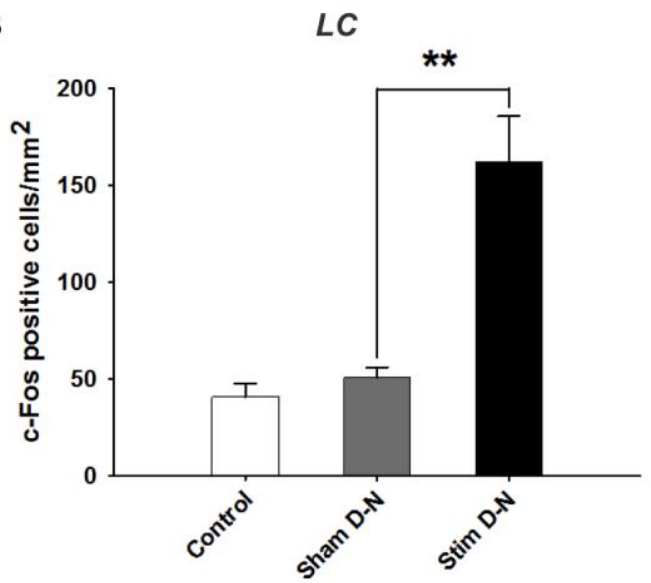

Figure 7. Expression of cFos immunoreactive cells in the PMC and LC. Cumulative data showing the means and S.E.M. of cFos expression for D-N animals in (A) PMC and (B) LC. Control $n=6$, Sham D-N $n=6$ and Stim D-N n $=7$. t-test, ${ }^{* *} p<0.005$ and ${ }^{* * *} p<0.001$

Effect of electrical stimulation of the urinary bladder on cFos immunoreactivity in the spinal cord

A quantitative assessment of cFos expression in the sacral parasympathetic nucleus (SPN), in the central canal region (DCM) and in laminae I and II of the dorsal horn (I-II) of the lumbosacral spinal cord (L6-S2) of D-N animals was done in a smaller sample size pilot study (Fig 8A; $n=2$ per experimental group). No increase was seen in these structures between control and sham groups (Control vs. Sham, t-test, NS, p>0.05; Figs 8B-D). However, cFos positive cells counts were significantly higher in stimulated compared to non-stimulated D-N animals (Sham vs. Stim, SPN, DCM and laminae I-II, ${ }^{* * *} \mathrm{p}<0.001,{ }^{*} \mathrm{p}=0.006$, and ${ }^{* *} \mathrm{p}=0.004$, respectively; Figs $8 \mathrm{~B}-\mathrm{D}$ ). 
A

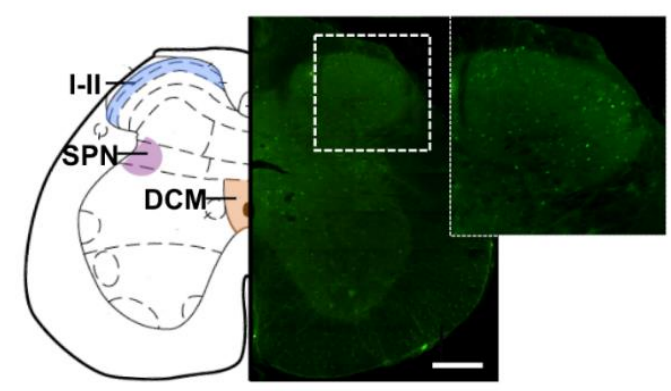

C

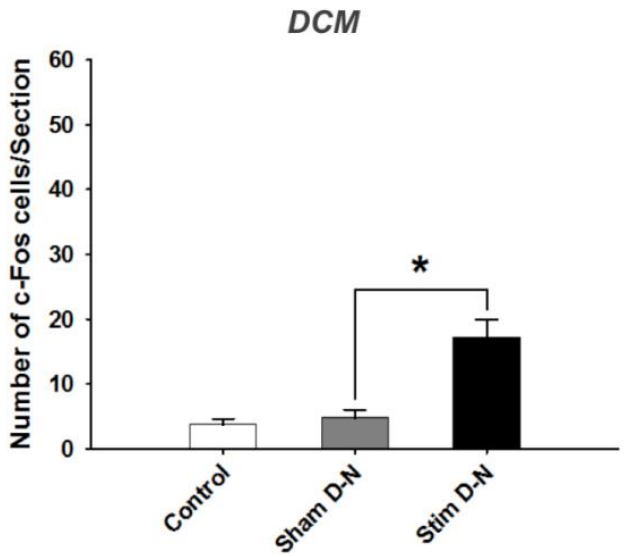

B

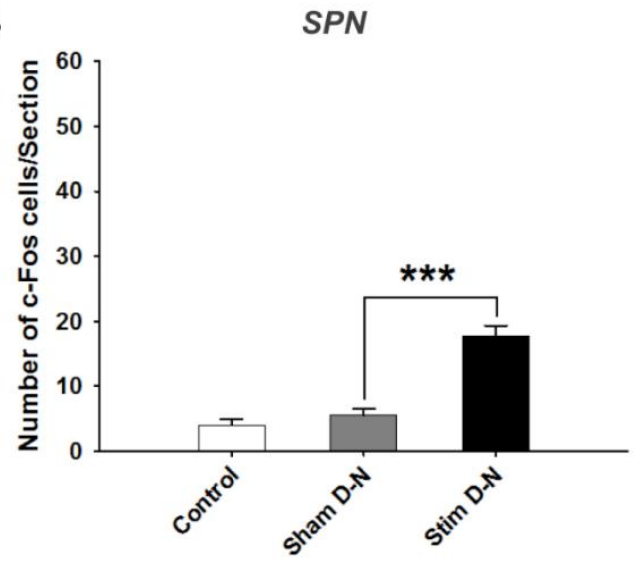

D

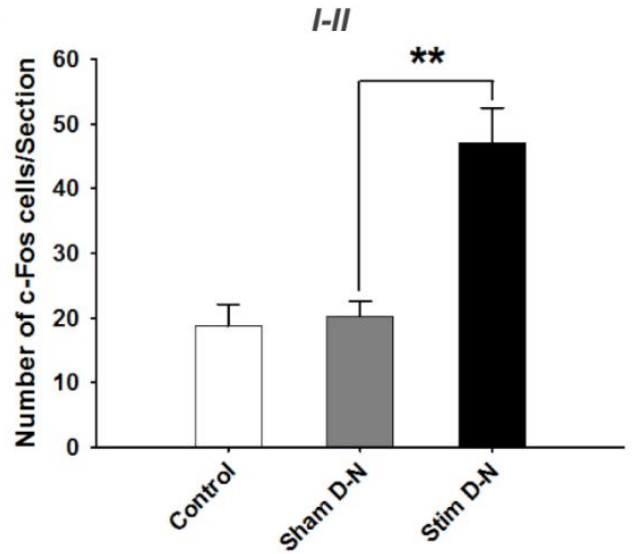

Figure 8. Expression of cFos immunoreactive cells in the lumbosacral spinal cord. (A) Coronal diagram of the rat lumbosacral spinal cord (L6) showing the location of the dorsal horn (i.e. laminae I and II), the sacral parasympathetic nucleus and the dorsal commissure (Rat brain atlas, Paxinos \& Watson, [60]) and a corresponding photomicrograph showing a spinal cord section stained for cFos of stimulated rat with bipolar stimulation electrode implanted in the bladder wall in the $D-N$ configuration. The higher power photomicrograph in the right corner shows the dorsal horn of the spinal cord. Abbreviations: I-II: laminae I and II, DCM: dorsal commissure, SPN: sacral parasympathetic nucleus. Scale bar $=200 \mu \mathrm{m}$. Cumulative data showing the means and S.E.M. for the optical density of cFos expression for D-N group in the (B) SPN, (C) DCM and (D) I-II of L6-S2 spinal cord. Control $n=4$, Sham $D-N n=4$ and Stim D-N $n=4$. t-test, ${ }^{*} p<0.05,{ }^{* *} p<0.005$ and $* * * \mathbf{p}<0.001$

Macroscopic evaluation of urinary bladder tissue and electrode implantation No sign of erosion, presence of excessive scar tissue or obvious change in tissue architecture in the urinary bladder and the surrounding tissues due to electrode implantation and chronic electrical stimulation performed during behavioral tests was revealed during post-mortem histological examination. Moreover, for all animals, the electrode wires remained correctly implanted in the bladder wall until the end of the experiments. 


\section{Discussion}

To our knowledge this study is the first that investigates the response of sensory afferent mechanosensitive fibers in the bladder in isolation in order to evaluate their distinct supraspinal neuronal response.

\section{Stimulation parameter choice}

It has been shown by tracing studies that the PAG receives projections from the sacral spinal cord. Also, electrophysiologically this connection has been established in several studies. Yet, the studies performed mostly made use of nociceptive stimuli to prove this connection. The majority of afferent signaling from the bladder is however related to its filling status, which is signaled by mechanosensitization from stretch-receptors. We have spent a fair amount of work into optimizing the stimulus used to mimic mechanosensitization, because it is essential for the interpretation of the results and draw solid scientific conclusions [45].

For the optimization of the stimulation parameters a duration of 30 seconds was used. Yet, for the final experiment we stimulated bladders for one hour. We did not evaluate the effect of long-lasting stimulation on bladder contractility. Yet, it is known from electrical skeletal muscle stimulation, that frequencies in the range of 20-50 Hz produce smooth contractions, that could be sustained over long periods [61,62]. Moreover, it has been shown, that high stimulation frequencies can cause the activation of motor neurons in the central nervous system. We aimed at triggering sensory fibers, which in turn could lead to the activation of motor neurons in the PMC. This will be discussed in more detail in the following paragraph. Low frequencies in combination with a short pulse width (around $500 \mu \mathrm{s}$ ) are less likely resulting in fatigue. In the case of $20 \mathrm{~Hz}$ stimulation it is unlikely, that a tetanus occurred, and that the bladder remained contracted during the stimulation phase. Tetany is generally known to occur at frequencies above $60 \mathrm{~Hz}$. So, in the present experiment we expect, that continuous contraction and relaxation occurred during the stimulation period in the form of detrusor 'twitches'.

Interestingly, somatosensory evoked potentials have been investigated in relation to different stimulation paradigms for anal sensory signaling. It has been shown, that low stimulation frequencies needed to be applied for longer durations in order to evoke the same somatosensory stimulation [63] than with higher frequencies. We therefore safely assume, that a stimulation period lasting one hour activated central perceptions. Moreover, low to intermediate frequencies are considered more appropriate to stimulate A $\delta$-fibers of the lower urinary tract $[43,64]$.

Both charge transfer and heat dissipation due to electrical current can contribute to cell injury at the electrode-tissue interface [65]. Thus, for minimal tissue damage during chronic stimulation, the amplitude should be set at a value causing minimum heat dissipation and charge transfer. Moreover, stimulation waveforms should be chargebalanced to reduce the possibility of tissue damage. In our case biphasic stimulation was considered most adequate. Intravesical pressure measurements showed that electrical 
stimulation with frequency of $20 \mathrm{~Hz}$ and amplitude of $2 \mathrm{~mA}$ was suitable and high enough to generate contractions of the urinary bladder, but these amplitudes did not cause voiding contractions.

As a critical note, one needs to realize that the simulation of a sensory stimulus is very challenging in rodents, because perceptions are nearly impossible to measure. However, studies investigating this in humans (i.e. functional magnetic resonance imaging) are very difficult to perform or miss the temporal or spatial resolution and therefore do not allow columnar analyses.

\section{Electrode position}

To evaluate PAG activity linked to urinary bladder sensation, the most appropriate parameter to stimulate the afferent nervous system of the lower urinary tract would be to initiate the sensation of bladder fullness by artificial bladder filling. However, functionally mapping of cFos needs to be applied carefully and critically [66], because cFos expression requires a prolonged and strong stimulation session, it is affected by anesthetic agents and can be induced non-specifically. Therefore, the introduction of a catheter for bladder filling, the measurement of bladder pressure and residual urine volume could induce cFos expression. This led us to opt for electrical stimulation of the urinary bladder in awake rats as a paradigm to study bladder sensation processing. Electrical stimulation is not a physiological stimulus, yet mimicking bladder sensation in a preclinical setting by electrical stimulation is a valid method. There is an added values of this stimulus for this type of sensory testing especially since it has been shown recently that afferent fibers detect bladder pressure or bladder wall stretch rather than bladder volume $[67,68]$.

For the current set of experiments a custom-made bipolar electrode construction has been designed to be chronically implanted into the bladder wall of rats. The position for the implantation of the two wires within the bladder wall was determined in the line with the general distribution of mechanosensitive afferent fibers in the rat bladder. The myelinated A $\delta$ fibers, described as afferent fibers responding to bladder filling and detrusor stretch and communicating filling sensation to the central nervous system, are mainly present in the smooth muscle layer of the urinary bladder $[17,69,70]$. Moreover, previous studies in rodents have revealed that the afferent fibers are notably more dense in the bladder neck and the dome [71]. Accordingly, we placed the two wires at the level of the neck on both sides for the N-N group and at the level of the neck and the dome for the D-N group, between the outer muscular and serosal layers of the urinary bladder wall.

From a theoretical point of view in the N-N configuration the electrodes are closer to the location that has a high density of afferent neurons. It is unclear however whether sensory afferent neurons located at the bladder neck are A $\delta$ or c-fibers. However, when stimulating a bigger area, which is the case in the D-N position, it is more likely that the whole detrusor muscle contracts rather than local contractions which leads to stretchinduced afferent activation of sensory neurons. However, as mentioned previously the stimulation parameters used -especially a pulse width of $0.5 \mathrm{~ms}$ - preferentially activate 
sensory afferents independently of their effect on contractility and or stretch. The difference in the density and eventually type of neurons in the different stimulation locations, might contribute to the differential activation of the rostral dlPAG and most columns of the caudal PAG, which did not respond to N-N but D-N stimulation. From a hypothetical point of view, activation stretch caused by bladder filling is exerting its effect on the whole detrusor muscle and does not occur at distinct locations such as the bladder neck. So theoretically speaking the D-N configuration seems to be more physiological. Based on these data not the density of neurons but rather the type and location in the bladder determine the activation of central nervous system structures. In the future, the identification of neurons that are responsible for PAG activation in transgenic animal models or in models with (transient) local lesion might reveal novel targeted treatment approaches for bladder disorders, with aberrant sensory processing.

\section{Behavioural findings}

There is a vast body of evidence that the PAG plays an important role in pain control [36, 72, 73]. Moreover, the dorsomedial and dorsolateral columns of the PAG have been demonstrated to be involved in anxiety-related behavior (dlPAG for the flight reaction and dmPAG for defensive behavior; $[37,74]$.

This raises the question whether electrical stimulation increases anxiety or pain and thus induced cFos expression in dorsal columns of the PAG. To exclude the possibility that cFos expression was induced by non-stimulation related effects, behavioral signs of pain and anxiety were analyzed in rats. There were no behavioural signs of pain nor did animals show anxiety-related behavior during the stimulation, which lead us to the conclusion, that cFos expression in the (dorsal) PAG, was not attributable to increased pain and anxiety.

However, in our study, an increase of urination frequency was found. In accordance with recent findings concerning afferents dysfunction in the development of bladder activity disorders such as OABS, the increased urinary frequency is likely to result from (hyper)excitation of the sensory system.

If -like applied in this experiment sensory afferent stimulation- results in a higher voiding frequency, this supports the hypothesis that OABS is in part related to faulty sensory signaling of bladder filling status. $\mathrm{OAB}$ is characterized by an increased frequency of voiding and urgency. Both are potentially related to faulty sensory signaling. From a neuromodulatory perspective it would be therefore interesting to test if it is possible by applying different stimulation parameters (i.e. high vs. low frequency) to ameliorate $\mathrm{OAB}$ symptoms by inhibiting sensory processing at the level of the bladder or even in the central nervous system.

Finally, before analyzing the results from cFos immunostaining, great care was taken to exclude that the observed changes were due to a displacement in the level of the electrode that would lead to unintentional stimulation of somatosensory receptors in the abdominal musculature and parietal peritoneum. 
Neuronal activation in the central nervous system upon electrical stimulation Previous studies showed a key role for the PAG in the regulatory system of urinary bladder [21-23, 25, 26, 29, 31, 34]. This structure is therefore seen as an interesting target to invoke treatment strategies to restore the normal function of the urinary bladder in urological disorders such as OABS. However, little is known about the relationship between the urinary bladder sensation and more specific PAG columnar activity.

The findings of the current set of experiments with regard to supraspinal activation upon sensory afferent stimulation are summarized in figure 9 .

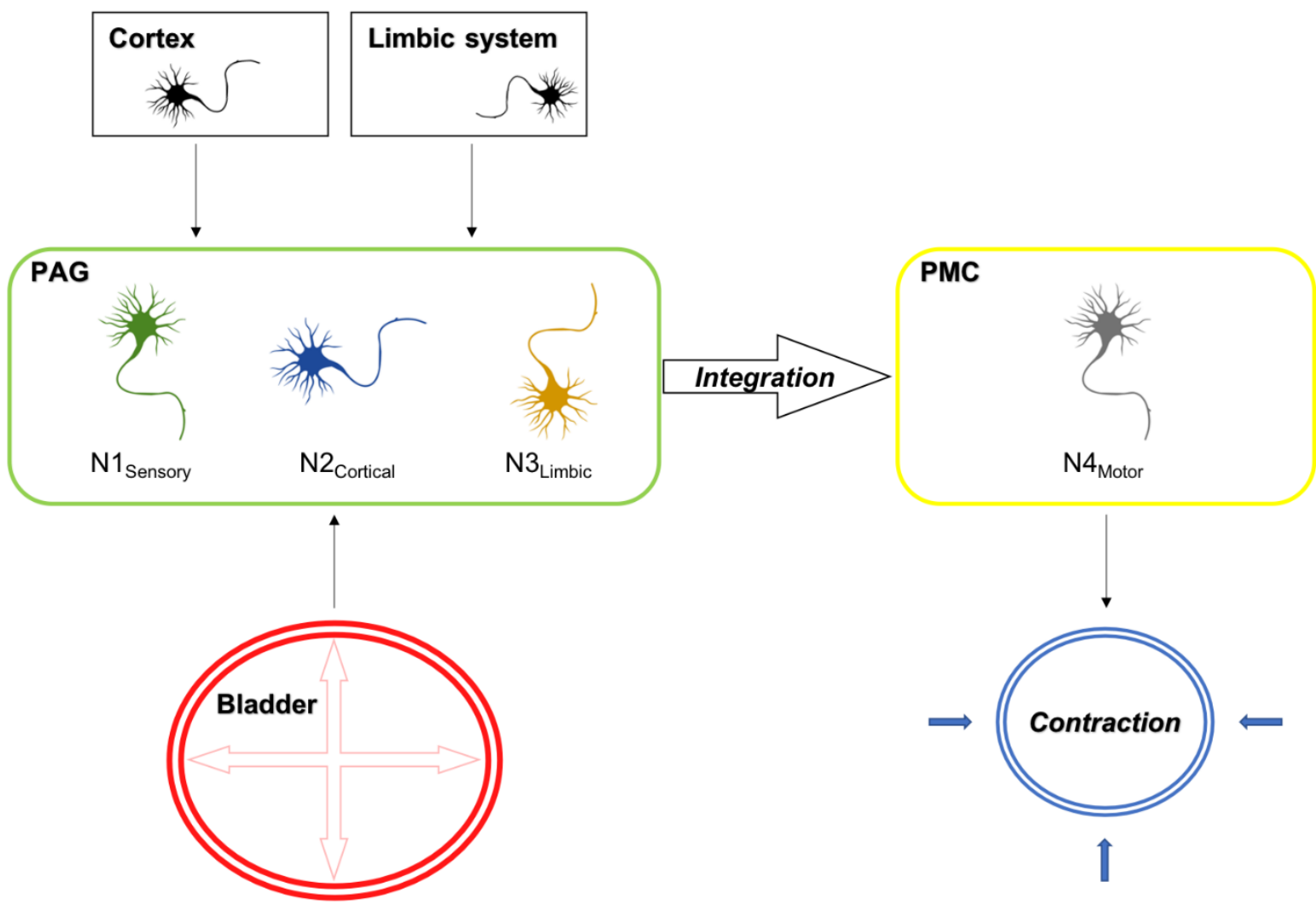

Figure 9. Electrical stimulation mimicking stretch of the detrusor muscle leads to the activation of sensory bladder afferents ( $A \delta$ fibers), which in turn activate sensory neurons in the PAG (N1 sensory). Besides, the PAG receives cortical and limbic input, which evaluates external circumstances, which either inhibit or facilitate voiding and activate cortical (N2 cortical) and limbic ( $\mathrm{N} 3$ limbic) projection neurons in the PAG. The integration of these signals takes places in neurons projecting to the PMC. In turn the PAG releases its disinhibitory action on the PMC, which results in the activation of efferent neurons (N4 motor), which result in the corresponding action at the spinal cord, leading to detrusor contractions and the relaxation of the external urethral sphincter, which are prerequisites for voiding. Also shown is the activation pattern as assessed by cFos expression in the D-N configuration for the rostral and caudal PAG. Red areas indicate activation and green areas are not activated upon sensory afferent stimulation. 


\section{Neuronal activation of the PAG by electrical stimulation}

Tracing studies have been performed earlier showing, that indeed there is a connection between the bladder and the PAG - more specially the ventrolateral column [75]. These results are in line with the current study. Whereas tracing studies are excellent to show neuroanatomical connection they cannot be used for functional connectivity assessment. The present study has the potential to combine neuroanatomical evidence with functional activation of neurons. To our knowledge there is no study investigating, which physiological stimulus activates neurons in brain structures such as the PAG. The studies that have been investigating (mainly by means of neurophysiological measurements) the activation of the PAG made use of noxious stimuli, which cannot be considered physiological. Considering that the PAG also plays a strong role in anxiety and painrelated functioning the use of noxious stimuli is biasing the results of earlier performed studies.

In control, Sham D-N and Sham N-N animals, few cFos immunoreactive cells were scattered throughout the PAG. These observations correspond to cFos basal expression and confirm that the presence of wires implanted in the bladder wall did not induce nonstimulation related cFos expression.

After electrical stimulation, the number of cFos positive cells was significantly greater than in non-stimulated animals. In the stimulated D-N group, cFos in the dorsomedial, lateral and ventrolateral regions of the caudal PAG was significantly increased compared to non-stimulated sham D-N group, with the biggest effect size in the vlPAG. Also, Marson showed, that the bladder projects to a very localized area in the vmPAG [76]. Even though, the terminology used (ventromedial vs. ventrolateral) is different the location and pattern of labeled neurons they show is identical with the activated area that we found. In their study they used a tracer that was injected into the EUS. This distinct localization in combination with the knowledge that neurons are connected to the EUS offers opportunities for selective treatment options. Even though, we did not observe obvious clusters of neurons in other columns it might be interesting in future experiments to identify if there are neuronal subpopulations within the columns of the PAG.

A previous study on cFos expression following activation of bladder afferents by noxious stimuli (acetic acid) to the urinary bladder reported cFos positive cells in the lateral area of the vlPAG [77]. The difference in columnar activation could be explained by the stimulation of different types of afferent fibers. Indeed, in the case of acetic acid infusion, mainly unmyelinated c-fibers respond to noxious stimuli [78]. Electrically evoked (submicturition threshold) contractions generated increased activity through central pathways that control the urinary bladder, initiated by activation of sensory axons. In previous studies, various stimulation protocols of the lower urinary tract caused changes in the central pathways such as PAG, PMC and spinal cord [77, 79-82].

It has been hypothesized, that the PAG receives information about the bladder filling status [83]. To our knowledge this study is the first study proofing the concept that 
afferent bladder signaling is indeed received by the PAG and functionally activates neurons.

Neuronal activation of the PMC by electrical stimulation

The PMC has a well-documented role in the control of micturition and urinary bladder function [18, 34, 84, 85]. It is known that activation of PMC initiates a complete synergic micturition response by inducing detrusor contractions and the relaxation of the external urethral sphincter resulting in micturition. Since the ventrolateral and lateral caudal PAG are known to project specifically to the PMC and activate its premotor interneurons [18, 30], activation of the PAG should result in dense cFos immunoreactive cells in PMC. Considering, that the PAG was activated minimally in the N-N configuration and the fact that activation of the PMC depends on PAG activation we investigated the expression of cFos in the D-N configuration only. CFos expression in the sham stimulated group showed a sparse and random distribution of cells expressing cFos whereas in D-N Stim group cFos positive cells were upregulated and consistently distributed.

Interestingly, we noticed in the nearby region, the LC, an increase in cFos immunoreactivity after electrical stimulation compared to non-stimulated animals presenting a low cFos level expression. Moreover, cFos positive neurons were primarily present in the dorsal half of the LC. We can conclude that the LC is activated upon bladder stimulation. Activation of LC neurons has been implicated in arousal and attention [86, 87]. In monkeys, cats and rats, sensory stimuli of various modalities can activate the LCnoradrenergic system [88-90]. Visceral stimuli increase LC discharge rate and are important in initiating a forebrain response [91, 92]. The LC has been previously identified to be connected anatomically to the bladder by means of tracing [76].

\section{Spinal cord activation}

Previous studies have revealed that the caudal lateral, ventrolateral and dorsal divisions of the PAG receive many projections from the lumbosacral cord and that stimulation of the afferent bladder nerves in the rat evokes short latency potentials in the most caudal part of the PAG $[85,93]$. Because neurons located in the laminae I and II, SPN and DCM of lumbosacral spinal cord project to the PAG, the visceroceptive inputs are likely to be propagated to the PAG through these spinal-PAG projections. We measured the cFos expression at the spinal levels of the L6-S2, since it corresponds to spinal entry sites of the pelvic nerve and to the regions of bladder afferent connections [70, 93, 94]. The present study demonstrated that bladder stimulation increases cFos expression in the superficial layers of the dorsal horn (laminae I and II), in the SPN and the central canal region in accordance with previous observations with noxious stimuli [80-82]. It should be noted that an increased cFos expression in the SPN may also be related to changes in the motor control of the vesical sphincters. The current study indicates strong neural activation consistent with mechanoceptive input in the spinal cord supports and validates the model of bladder electrical stimulation. 
We optimized the parameters for the stimulation so that an intravesical pressure would occur caused by detrusor contractility in the absence of voiding. We applied the stimulation directly to the bladder muscle through the implanted electrode. There is no reason to believe that direct stimulation at the detrusor muscle itself causes the activation of efferent motor neurons in the central nervous system directly. Likely, efferent motor neurons were activated as a consequence of the bladder-spinal cord reflex network. One example of this is the guarding reflex, which occurs once there is a sudden rise in abdominal pressure in order to prevent leakage of urine resulting in the contraction of the EUS to prevent urine from leaking. Augmentations of intravesical pressure can lead to either the activation of the guarding reflex (short reflex loop at the level of the spinal cord) in order to prevent leakage of urine or the initiation of voiding (long reflex loop mediated via the brain).

In the spinal cord, the efferent system is exclusively located in the ventral horn, whereas the sensory part is located in the dorsal horn. Furthermore, motor neurons can be easily distinguished in the spinal cord due to their size and shape. It is indeed true that some motoneurons stained positive for cFos (see Fig 8A, which shows incidentally a motoneuron stained in the ventral horn). Due to the fact that we quantified cFos positive neurons in the dorsal part of the spinal cord only, we can surely say that these are related to sensory activity.

This means that we could (i) proof that sensory activation took place in our setup (ii) exclusively focus on the sensory component by delineating and quantifying a neuroanatomical region, which is supposedly only involved in sensory information processing. As we were unable to assess if animals were aware of a sensory perception we proof a sensory component in our experiments by the activation of sensory neurons in a region of the spinal cord that is exclusively mediating sensory signals.

\section{Technical considerations}

For the purpose of identifying sensory processing upon sensory bladder related signals in the central nervous system, we used immunohistochemical detection of the cFos protein in the CNS of rats after urinary bladder electrical stimulation.

It is important to realize that cFos expression is a marker of neuronal activity but not a marker for the efferent result (such as the contraction of a muscle) or the effect on a distant neuronal target (such as the PMC, which is under the control of the PAG). The activation of inhibitory neurons (i.e. GABAergic interneurons) results in distinctive actions at the distant side than the activation of excitatory neurons. In this study, we did not differentiate between the type of neuron that was activated. However, we did followup on this question in a study, which was recently published studying the co-expression of cFos and neuronal markers in the PAG [95]. Also, like described in the above diagram, due to input of various regions to the PAG, we cannot make a difference which neuronal activation resulted from afferent sensory or cortico-limbic inputs. 
Staining of cFos does not allow to differentiate in temporal terms. Therefore, our study does not allow to differentiate the temporal sequence in which neuronal activation occurred. Even stronger rather than considering PAG columns as isolated entities it is possible that those interact and modulate their function. For instance, Noto (1991) showed that stimulation of the pelvic nerve afferents induced evoked-potentials in the dorsal PAG. The optimal site for inducing bladder contractions was in the ventral PAG. This suggests, that afferent and efferent pathways are distinct in the PAG. Based on their results they suggest, that it is unlikely that the PAG is a relay station, but raise the possibility that the dorsal part of the PAG may have modulatory effects on micturition [85].

Moreover, it appeared that the location of the stimulation activated the caudal, but not the rostral (dl)PAG. The meaning of this differential activation is unknown. The focus with regard to functional differences in the PAG has mostly been on the columnar structure rather than functional difference in the rostro-caudal direction. It has been shown, that specific projections from one PAG column to other brain areas (i.e. rostral ventromedial medulla) differ in the rostro-caudal axis [96].

These regional differences cannot be investigated by exposure to noxious compounds because they are not applied in distinct parts of the bladder. Therefore, our experimental setup provides unique insights into the columnar activation of the PAG.

\section{Future perspectives}

To characterize and elucidate the function of the receptors involved in the activated neurons playing a role in the propagation of signals generated by the bladder, additional investigations by immunohistochemistry and in vivo electrophysiological experiments are required. Since several levels of the central nervous system are involved in the control of micturition, a cFos study in structures such as the prefrontal cortex, the insula and the anterior cingulate cortex should be considered to evaluate their potential influence in the processing of urinary bladder sensation elicited by electrical stimulation.

A deep understanding of complex neural circuits controlling the urinary bladder can be offer opportunities for novel modalities for OABS. Among the innovative therapeutic approaches to restore the correct functioning of the urinary bladder in OABS patients, deep brain stimulation (DBS) seems to be an attractive alternative treatment modality $[97,98]$. 


\section{Acknowledgments}

Authors gratefully acknowledge T. Camps and J. Robinet from Medtronic for providing pacing wires. Authors thank HE. Steinbusch and M. Philippens for their technical support and Dr. M. Lagière for her assistance with the statistical analysis of the results.

\section{Disclosure of potential conflicts of interest}

Dr. C. Meriaux is a Marie Skłodowska-Curie postdoctoral fellow. This study was funded by the EU - FP7 Marie Skłodowska-Curie ITN - TRUST program. All other authors declare that they have no conflict of interest.

\section{Research involving Animals}

All procedures performed in studies involving animals were in accordance with the ethical standards of the institution or practice at which the studies were conducted. 


\section{References}

1. Hashim H, Abrams P (2007) Overactive bladder: an update. Curr Opin Urol 17:231-236. doi: 10.1097/MOU.0b013e32819ed7f9

2. Irwin DE, Milsom I, Hunskaar S, et al (2006) Population-Based Survey of Urinary Incontinence, Overactive Bladder, and Other Lower Urinary Tract Symptoms in Five Countries: Results of the EPIC Study. Eur Urol 50:1306-1315 . doi: 10.1016/j.eururo.2006.09.019

3. Stewart WF, Van Rooyen JB, Cundiff GW, et al (2003) Prevalence and burden of overactive bladder in the United States. World J Urol 20:327-36 . doi: 10.1007/s00345-002-0301-4

4. Abrams P, Cardozo L, Fall M, et al (2003) The standardisation of terminology in lower urinary tract function: report from the standardisation sub-committee of the International Continence Society. Urology 61:37-49 . doi: 10.1016/S0090-4295(02)02243-4

5. Onukwugha E, Zuckerman IH, McNally D, et al (2009) The total economic burden of overactive bladder in the United States: a disease-specific approach. Am J Manag Care 15:S90-7

6. Madhuvrata P, Singh M, Hasafa Z, Abdel-Fattah M (2012) Anticholinergic Drugs for Adult Neurogenic Detrusor Overactivity: A Systematic Review and Meta-analysis. Eur Urol 62:816-830 . doi: 10.1016/j.eururo.2012.02.036

7. Veenboer PW, Bosch JLHR (2014) Long-Term Adherence to Antimuscarinic Therapy in Everyday Practice: A Systematic Review. J Urol 191:1003-1008 . doi: 10.1016/j.juro.2013.10.046

8. Giannantoni A, Proietti S, Costantini E, et al (2015) OnabotulinumtoxinA intravesical treatment in patients affected by overactive bladder syndrome: best practice in real-life management. Urologia 82:179-83 . doi: 10.5301/uro.5000120

9. Mangera A, Apostolidis A, Andersson KE, et al (2014) An Updated Systematic Review and Statistical Comparison of Standardised Mean Outcomes for the Use of Botulinum Toxin in the Management of Lower Urinary Tract Disorders. Eur Urol 65:981-990 . doi: 10.1016/j.eururo.2013.10.033

10. Chapple C, Sievert K-D, MacDiarmid S, et al (2013) OnabotulinumtoxinA $100 \mathrm{U}$ Significantly Improves All Idiopathic Overactive Bladder Symptoms and Quality of Life in Patients with Overactive Bladder and Urinary Incontinence: A Randomised, Double-Blind, Placebo-Controlled Trial. Eur Urol 64:249-256 . doi: 10.1016/j.eururo.2013.04.001

11. Nitti VW, Dmochowski R, Herschorn S, et al (2013) OnabotulinumtoxinA for the Treatment of Patients with Overactive Bladder and Urinary Incontinence: Results of a Phase 3, Randomized, Placebo Controlled Trial. J Urol 189:2186-2193 . doi: 10.1016/j.juro.2012.12.022

12. Sukhu T, Kennelly M, Kurpad R (2016) Sacral neuromodulation in overactive bladder: a review and current perspectives. Res Reports Urol Volume 8:193-199 . doi: 10.2147/RRU.S89544 
13. Peters KM, MacDiarmid SA, Wooldridge LS, et al (2009) Randomized Trial of Percutaneous Tibial Nerve Stimulation Versus Extended-Release Tolterodine: Results From the Overactive Bladder Innovative Therapy Trial. J Urol 182:1055-1061 . doi: 10.1016/j.juro.2009.05.045

14. Peters KM, Carrico DJ, Perez-Marrero RA, et al (2010) Randomized Trial of Percutaneous Tibial Nerve Stimulation Versus Sham Efficacy in the Treatment of Overactive Bladder Syndrome: Results From the SUmiT Trial. J Urol 183:1438-1443 . doi: 10.1016/j.juro.2009.12.036

15. Reyblat P, Ginsberg DA (2008) Augmentation cystoplasty: what are the indications? Curr Urol Rep 9:452-8

16. De Groat WC, Griffiths D, Yoshimura N (2015) Neural Control of the Lower Urinary Tract. Compr Physiol 5:327-96 . doi: 10.1002/cphy.c130056

17. Birder L, De Groat W, Mills I, et al (2010) Neural Control of the Lower Urinary Tract: Peripheral and Spinal Mechanisms. Neurourol Urodyn 29:128-39 . doi: $10.1002 /$ nau.20837

18. Fowler CJ, Griffiths D, de Groat WC (2008) The neural control of micturition. Nat Rev Neurosci 9:453-66 . doi: 10.1038/nrn2401

19. Fowler CJ (2006) Integrated control of lower urinary tract - clinical perspective. $\mathrm{Br} \mathrm{J}$ Pharmacol 147:14-24 . doi: 10.1038/sj.bjp.0706629

20. Abrams P (2003) Describing bladder storage function: overactive bladder syndrome and detrusor overactivity. Urology 62:28-37 . doi: 10.1016/j.urology.2003.09.050

21. Blok BFM, Willemsen ATM, Holstege G (1997) A PET study on brain control of micturition in humans. braini0110 Brain 120:111-121

22. Blok BFM, Sturms LM, Holstege G (1998) Brain activation during micturition in women. Brain 121:2033-2042

23. Griffiths D, Derbyshire S, Stenger A, Resnick N (2005) Brain control of normal and overactive bladder. J Urol 174:1862-1867 . doi: 10.1097/01.ju.0000177450.34451.97

24. Griffiths D, Tadic SD (2008) Bladder control, urgency, and urge incontinence: Evidence from functional brain imaging. Neurourol Urodyn 27:466-474 . doi: 10.1002/nau.20549

25. Athwal BS, Berkley KJ, Hussain I, et al (2001) Brain responses to changes in bladder volume and urge to void in healthy men. Brain 124:369-77

26. Tai C, Wang J, Jin T, et al (2009) Brain Switch for Reflex Micturition Control Detected by fMRI in Rats. J Neurophysiol 102:2719-2730 . doi: 10.1152/jn.00700.2009

27. Vizzard MA, Erickson VL, Card JP, et al (1995) Transneuronal labeling of neurons in the adult rat brainstem and spinal cord after injection of pseudorabies virus into the urethra. J Comp Neurol 355:629-640 . doi: 10.1002/cne.903550411

28. Fowler CJ, Griffiths DJ (2009) A decade of functional brain imaging applied to bladder control. Neurourol Urodyn 29:49-55 . doi: 10.1002/nau.20740 
29. Matsuura S, Downie JW, Allen G V (2000) Micturition evoked by glutamate microinjection in the ventrolateral periaqueductal gray is mediated through Barrington's nucleus in the rat. Neuroscience 101:1053-61

30. Blok BF, Holstege G (1994) Direct projections from the periaqueductal gray to the pontine micturition center (M-region). An anterograde and retrograde tracing study in the cat. Neurosci Lett 166:93-6

31. Taniguchi N, Miyata M, Yachiku S, et al (2002) A study of micturition inducing sites in the periaqueductal gray of the mesencephalon. J Urol 168:1626-31 . doi: 10.1097/01.ju.0000030157.79336.5d

32. Blok BFM, De Weerd H, Holstege G (1995) Ultrastructural evidence for a paucity of projections from the lumbosacral cord to the pontine micturition center or M-region in the cat: A new concept for the organization of the micturition reflex with the periaqueductal gray as central relay. J Comp Neurol 359:300-309 . doi: 10.1002/cne.903590208

33. Mantyh PW (1982) Forebrain projections to the periaqueductral gray in the monkey, with observations in the cat and rat. J Comp Neurol 206:146-158 . doi: 10.1002/cne.902060205

34. Griffiths DJ, Fowler CJ (2013) The micturition switch and its forebrain influences. Acta Physiol 207:93-109. doi: 10.1111/apha.12019

35. Griffiths D, Tadic SD, Schaefer W, Resnick NM (2007) Cerebral control of the bladder in normal and urge-incontinent women. Neuroimage 37:1-7 . doi: 10.1016/j.neuroimage.2007.04.061

36. Bandler R, Shipley MT (1994) Columnar organization in the midbrain periaqueductal gray: modules for emotional expression? Trends Neurosci 17:379-89

37. Carrive P (1993) The periaqueductal gray and defensive behavior: functional representation and neuronal organization. Behav Brain Res 58:27-47

38. Linnman C, Moulton EA, Barmettler G, et al (2012) Neuroimaging of the periaqueductal gray: State of the field. Neuroimage 60:505-522 . doi: 10.1016/j.neuroimage.2011.11.095

39. Beitz AJ (1985) The midbrain periaqueductal gray in the rat. I. Nuclear volume, cell number, density, orientation, and regional subdivisions. J Comp Neurol 237:445-459 . doi: 10.1002/cne.902370403

40. Menant O, Andersson F, Zelena D, Chaillou E (2016) The benefits of magnetic resonance imaging methods to extend the knowledge of the anatomical organisation of the periaqueductal gray in mammals. J Chem Neuroanat 77:110-120 . doi: 10.1016/j.jchemneu.2016.06.003

41. Madersbacher H (1990) Intravesical electrical stimulation for the rehabilitation of the neuropathic bladder. Paraplegia 28:349-352 . doi: 10.1038/sc.1990.47

42. Streng T, Hedlund $\mathrm{P}$, Talo A, et al (2006) Phasic non-micturition contractions in the bladder of the anaesthetized and awake rat. BJU Int 97:1094-1101 . doi: 10.1111/j.1464410X.2006.06137.x

43. Jiang CH (1998) Modulation of the micturition reflex pathway by intravesical electrical 
stimulation: an experimental study in the rat. Neurourol Urodyn 17:543-53

44. Ebner A, Jiang C, Lindström S (1992) Intravesical electrical stimulation--an experimental analysis of the mechanism of action. J Urol 148:920-4

45. Buyle S, Wyndaele JJ, D’Hauwers K, et al (1998) Optimal parameters for transurethral intravesical electrostimulation determined in an experiment in the rat. Eur Urol 33:50710

46. Tsui BC (2008) Electrical nerve stimulation. In: Tsui B, Chan V, Finucane B, et al (eds) Atlas of ultrasound- and nerve stimulation-guided regional anesthesia, XXIII. Springer New York, pp 9-18

47. Jiang CH, Lindström S (1996) Intravesical electrical stimulation induces a prolonged decrease in micturition threshold volume in the rat. J Urol 155:1477-81

48. Biallosterski BT, Prickaerts J, Rahnama'i MS, et al (2015) Changes in voiding behavior in a mouse model of Alzheimer's disease. Front Aging Neurosci 7:160 . doi: 10.3389/fnagi.2015.00160

49. Haller J, Tóth M, Halasz J, De Boer SF (2006) Patterns of violent aggression-induced brain c-fos expression in male mice selected for aggressiveness. Physiol Behav 88:173-182 . doi: 10.1016/j.physbeh.2006.03.030

50. Rodella L, Rezzani R, Gioia M, et al (1998) Expression of Fos immunoreactivity in the rat supraspinal regions following noxious visceral stimulation. Brain Res Bull 47:357-366 . doi: 10.1016/S0361-9230(98)00123-3

51. Arout CA, Caldwell M, McCloskey DP, Kest B (2014) C-Fos activation in the periaqueductal gray following acute morphine-3 $\beta$-d-glucuronide or morphine administration. Physiol Behav 130:28-33 . doi: 10.1016/j.physbeh.2014.02.056

52. Baulmann J, Spitznagel H, Herdegen T, et al (2000) Tachykinin receptor inhibition and cFos expression in the rat brain following formalin-induced pain. Neuroscience 95:813-20

53. Somogyi P, Takagi H (1982) A note on the use of picric acid-paraformaldehydeglutaraldehyde fixative for correlated light and electron microscopic immunocytochemistry. Neuroscience 7:1779-83

54. Tang Y, Liu L, Xu D, et al (2018) Interaction between astrocytic colony stimulating factor and its receptor on microglia mediates central sensitization and behavioral hypersensitivity in chronic post ischemic pain model. Brain Behav Immun 68:248-260 . doi: $10.1016 /$ j.bbi.2017.10.023

55. Spitzer N, Sammons GS, Price EM (2011) Autofluorescent cells in rat brain can be convincing impostors in green fluorescent reporter studies. J Neurosci Methods 197:4855 . doi: 10.1016/j.jneumeth.2011.01.029

56. Schnell SA, Staines WA, Wessendorf MW (1999) Reduction of lipofuscin-like autofluorescence in fluorescently labeled tissue. J Histochem Cytochem 47:719-30 . doi: $10.1177 / 002215549904700601$

57. Clancy B, Cauller LJ (1998) Reduction of background autofluorescence in brain sections 
following immersion in sodium borohydride. J Neurosci Methods 83:97-102

58. Lawrenson C, Koutsikou S, Lumb B, Apps R (2017) S191 Cerebellar contributions to fear behaviour. Clin Neurophysiol 128:e239 . doi: 10.1016/J.CLINPH.2017.07.199

59. Hescham S, Jahanshahi A, Meriaux C, et al (2015) Behavioral effects of deep brain stimulation of different areas of the Papez circuit on memory- and anxiety-related functions. Behav Brain Res 292:353-360 . doi: 10.1016/j.bbr.2015.06.032

60. Paxinos G, Watson C (2007) The rat brain in stereotaxic coordinates. Elsevier

61. Doucet BM, Lam A, Griffin L (2012) Neuromuscular electrical stimulation for skeletal muscle function. Yale J Biol Med 85:201-15

62. Bhadra N, Peckham PH (1997) Peripheral nerve stimulation for restoration of motor function. J Clin Neurophysiol 14:378-93

63. Evers J, Devane L, Carrington E V., et al (2014) Effects of stimulation frequency and intensity in sacral neuromodulation on anorectal inputs to the somatosensory cortex in an experimental model. Br J Surg 101:1317-1328 . doi: 10.1002/bjs.9587

64. Langille M, Gonzalez-Cueto JA, Sundar S (2008) Analysis of the selective nature of sensory nerve stimulation using different sinusoidal frequencies. Int J Neurosci 118:1131-44 . doi: $10.1080 / 00207450701769323$

65. Crago PE, Peckham PH, Mortimer JT, Van der Meulen JP (1974) The choice of pulse duration for chronic electrical stimulation via surface, nerve, and intramuscular electrodes. Ann Biomed Eng 2:252-64

66. Dragunow M, Faull R (1989) The use of c-fos as a metabolic marker in neuronal pathway tracing. J Neurosci Methods 29:261-5

67. Andersson K-E (2015) Purinergic signalling in the urinary bladder. Auton Neurosci 191:78-81 . doi: 10.1016/j.autneu.2015.04.012

68. Janssen DAW, Schalken JA, Heesakkers JPFA (2017) Urothelium update: how the bladder mucosa measures bladder filling. Acta Physiol 220:201-217 . doi: 10.1111/apha.12824

69. Iggo A (1955) Tension receptors in the stomach and the urinary bladder. J Physiol 128:593-607

70. Xu L, Gebhart GF (2008) Characterization of Mouse Lumbar Splanchnic and Pelvic Nerve Urinary Bladder Mechanosensory Afferents. J Neurophysiol 99:244-253 . doi: 10.1152/jn.01049.2007

71. Grol S, van Koeveringe GA, de Vente J, et al (2008) Regional differences in sensory innervation and suburothelial interstitial cells in the bladder neck and urethra. BJU Int 102:870-877 . doi: 10.1111/j.1464-410X.2008.07752.x

72. Rosen SD, Paulesu E, Frith CD, et al (1994) Central nervous pathways mediating angina pectoris. Lancet (London, England) 344:147-50

73. Young RF, Chambi VI (1987) Pain relief by electrical stimulation of the periaqueductal and periventricular gray matter. J Neurosurg 66:364-371 . doi: 10.3171/jns.1987.66.3.0364 
74. Keay KA, Bandler R (2001) Parallel circuits mediating distinct emotional coping reactions to different types of stress. Neurosci Biobehav Rev 25:669-78

75. Morrison JF (1997) The physiological mechanisms involved in bladder emptying. Scand J Urol Nephrol Suppl 184:15-8

76. Marson L (1997) Identification of central nervous system neurons that innervate the bladder body, bladder base, or external urethral sphincter of female rats: a transneuronal tracing study using pseudorabies virus. J Comp Neurol 389:584-602

77. Mitsui T, Kakizaki H, Matsuura S, et al (2003) Chemical bladder irritation provokes c-fos expression in the midbrain periaqueductal gray matter of the rat. Brain Res 967:81-8

78. Aizawa N, Fujimori Y, Kobayashi J, et al (2018) KPR-2579, a novel TRPM8 antagonist, inhibits acetic acid-induced bladder afferent hyperactivity in rats. Neurourol Urodyn. doi: $10.1002 /$ nau.23532

79. Yamada Y, Ukimura O, Liu G, et al (2012) Efficacy of Neuroselective and Site-specific Nociceptive Stimuli of Rat Bladder. Urology 79:483.e7-483.e12 . doi: 10.1016/j.urology.2011.10.006

80. Birder LA, de Groat WC (1992) Increased c-fos expression in spinal neurons after irritation of the lower urinary tract in the rat. J Neurosci 12:4878-89

81. Birder LA, Roppolo JR, Erickson VL, de Groat WC (1999) Increased c-fos expression in spinal lumbosacral projection neurons and preganglionic neurons after irritation of the lower urinary tract in the rat. Brain Res 834:55-65

82. Mitsui T, Kakizaki H, Matsuura S, et al (2001) Afferent fibers of the hypogastric nerves are involved in the facilitating effects of chemical bladder irritation in rats. J Neurophysiol 86:2276-84

83. Griffiths D, Holstege G, Dalm E, Wall H De (1990) Control and coordination of bladder and urethral function in the brainstem of the cat. Neurourol Urodyn 9:63-82 . doi: 10.1002/nau.1930090108

84. Noto H, Roppolo JR, Steers WD, de Groat WC (1989) Excitatory and inhibitory influences on bladder activity elicited by electrical stimulation in the pontine micturition center in the rat. Brain Res 492:99-115

85. Noto H, Roppolo JR, Steers WD, de Groat WC (1991) Electrophysiological analysis of the ascending and descending components of the micturition reflex pathway in the rat. Brain Res 549:95-105

86. Aston-Jones G (1985) Behavioral functions of locus coeruleus derived from cellular attributes. Physiol Psychol 13:118-126 . doi: 10.3758/BF03326513

87. Foote SL, Bloom FE, Aston-Jones G (1983) Nucleus locus ceruleus: new evidence of anatomical and physiological specificity. Physiol Rev 63:844-914

88. Foote SL, Aston-Jones G, Bloom FE (1980) Impulse activity of locus coeruleus neurons in awake rats and monkeys is a function of sensory stimulation and arousal. Proc Natl Acad Sci U S A 77:3033-7 
89. Abercrombie ED, Jacobs BL (1987) Single-unit response of noradrenergic neurons in the locus coeruleus of freely moving cats. I. Acutely presented stressful and nonstressful stimuli. J Neurosci 7:2837-43

90. Aston-Jones G, Bloom FE (1981) Norepinephrine-containing locus coeruleus neurons in behaving rats exhibit pronounced responses to non-noxious environmental stimuli. J Neurosci 1:887-900

91. Elam M, Thorén P, Svensson TH (1986) Locus coeruleus neurons and sympathetic nerves: activation by visceral afferents. Brain Res 375:117-25

92. Page ME, Akaoka H, Aston-Jones G, Valentino RJ (1992) Bladder distention activates noradrenergic locus coeruleus neurons by an excitatory amino acid mechanism. Neuroscience 51:555-63

93. Ding YQ, Zheng HX, Gong LW, et al (1997) Direct projections from the lumbosacral spinal cord to Barrington's nucleus in the rat: a special reference to micturition reflex. J Comp Neurol 389:149-60

94. Morgan C, Nadelhaft I, de Groat WC (1981) The distribution of visceral primary afferents from the pelvic nerve to Lissauer's tract and the spinal gray matter and its relationship to the sacral parasympathetic nucleus. J Comp Neurol 201:415-440 . doi: 10.1002/cne.902010308

95. Zare A, Jahanshahi A, Meriaux C, et al (2018) Glutamatergic cells in the periaqueductal gray matter mediate sensory inputs after bladder stimulation in freely moving rats. Int J Urol. doi: 10.1111/iju.13562

96. Loyd DR, Murphy AZ (2009) The role of the periaqueductal gray in the modulation of pain in males and females: are the anatomy and physiology really that different? Neural Plast 2009:462879 . doi: 10.1155/2009/462879

97. Green AL, Stone E, Sitsapesan H, et al (2012) Switching off micturition using deep brain stimulation at midbrain sites. Ann Neurol 72:144-147 . doi: 10.1002/ana.23571

98. Stone E, Coote JH, Lovick TA (2015) Effect of electrical vs. chemical deep brain stimulation at midbrain sites on micturition in anaesthetized rats. Acta Physiol 214:135-145 . doi: 10.1111/apha.12491 


\section{Chapter 5}

Glutamatergic cells in the periaqueductal gray matter mediate sensory inputs following bladder stimulation in freely moving rats

Aryo Zare, Ali Jahanshahi, Celine Meriaux, Harry W. M. Steinbusch, Gommert A. van Koeveringe

Int J Urol. doi:10.1111/iju.13562 


\begin{abstract}
Objectives

The periaqueductal gray matter (PAG) of the midbrain mediates various important functions ranging from autonomic to emotional domains namely fear/anxiety, pain and micturition control. The PAG is organized in functionally distinct columns. Previous studies revealed that the ventrolateral PAG (vlPAG) is the column mostly engaged in sensorimotor bladder control. In the current study we aimed to phenotype the vlPAG neurons following bladder stimulation.
\end{abstract}

\title{
Methods
}

In the experimental group electrical stimulation of the bladder was conducted in freely moving condition via a bipolar stimulation electrode implanted in the bladder wall. Thereafter, the brain sections were processed for immunehistochemical analysis using antibodies against c-Fos (neuronal activation marker) together with one of the following: tyrosine hydroxylase (TH, dopaminergic cell marker), vesicular glutamate transporter (VGLUT2, glutamatergic cell marker), serotonin, glutamate decarboxylase (GAD67, GABA cell marker) and neuronal nitric oxide synthase (nNOS). We used designed based confocal stereological analysis to quantify the immunohistochemically stained sections.

\section{Results}

In line with previous reports, we found a significant increase in the number of c-Fos cells in the vlPAG. Moreover the ratio of c-Fos cells double labeled with VGLUT2 was significantly higher in the vIPAG region in the stimulated compared to the sham group. Quantitative analysis of the other cell types did not reveal any significant difference.

\section{Conclusion}

These findings suggest that glutamatergic neurotransmission in the vlPAG is seemingly the main pathway to be activated after receiving sensory signals from the bladder.

\section{Key Words:}

Glutamic Acid; Neurotransmitter; periaqueductal gray; Urinary bladder

\section{Abbreviations and Acronyms}

periaqueductal gray matter (PAG)

pontine micturition center (PMC)

ventrolateral column of PAG (vlPAG)

dorsolateral column of PAG(dIPAG)

neuronal nitric oxide synthase (nNOS) 
tyrosine hydroxylase $(\mathrm{TH})$

vesicular glutamate transporter (VGLUT)

glutamate decarboxylase (GAD)

5-hydroxytriptamine (5-HT)

\section{Introduction}

The periaqueductal gray matter (PAG) is the central gray matter of the midbrain in continuance of the circumventricular organs and is somehow analogous to the gray matter of the spinal cord. Due to its axial location, it is involved in various important functions, ranging from autonomic to emotional domains namely fear/anxiety, pain and micturition control. Its role in the control of the bladder function encompasses both downstream connections as well as connections with the higher brain centers involved in decision making. The PAG has been found to bear functionally separate columns which are also histologically separate in human brain. There exist three pairs of columns namely ventrolateral, lateral and dorsolateral as well as a single dorsomedial column.

Studies in the cat brain have shown $30 \%$ of the PAG neurons to be positively labelled for glutamate[1]. The PAG has the capacity to synthetize[2] serotonin, dopamine[3], nNOS[4] and GABA[5, 6]. nNOS is the neuronal isoform of the enzyme nitric oxide synthase which generates the neurotransmitter nitric oxide in the nervous tissue. No particular relations between the cell types, their neurotransmitter contents, functions and projections have been discovered so far. Electrophysiologic studies had discovered the role of PAG in neural control of micturition[7]. Previous studies have defined ventrolateral column of PAG (vlPAG) as the column responsible for sensorimotor communication with bladder. c-Fos, a transcription factor expressed after neuronal activation, was significantly higher in vlPAG of rats with chemical irritated bladder relative to saline infused group [8]. Electrical stimulation of the pelvic nerves in the cat, evoked maximum field potentials in vlPAG[9]. Studies in cats confirm that the electrical or chemical (DL-homocysteine) stimulation of vlPAG results in contraction of the bladder[10]. Chemical stimulation of the vlPAG by D,Lhomocysteic acid in rats increased the frequency of micturition[11]. Stereotaxic injections of the inhibitory mediator cobalt chloride into the caudal vlPAG reversibly attenuated bladder contractions and external urethral sphincter electromyographic activity in rats[12]. Injection of other inhibitory or stimulatory agents, $\mathrm{CoCl} 2$ \& lglutamate into the vIPAG of rats resulted suppression or stimulation of micturition, respectively[13].

In this study we aimed to identify which cell types in the vIPAG relay sensory inputs from the bladder. To this end, we conducted designed-based stereology and confocal microscopy on major stimulatory and inhibitory neuronal cell types in the PAG following 
electrical stimulation of the bladder wall. However, using c-Fos as a neuronal activation marker as described in this paper has some shortcomings including alteration by anesthetic effect. Therefore, we performed studies on freely moving animals with implanted electrodes after a 2 week recovery period.

\section{Methods}

\section{Animals}

Adult male Sprague Dawley rats (body weight 300-350g at the time of surgery) were obtained from the central animal facility (Maastricht University, Netherlands). The animals were divided into two groups: the experimental group $(n=6)$ underwent electrode implantation in the bladder and the other group $(n=4)$ served as sham operated controls. The experimental procedures were approved by the Dutch Committee for Animal Research local ethical committee, Maastricht University.

\section{Surgical procedure and electrode implantation}

In order to perform electrical stimulation of the urinary bladder in rats, a custom made construction was built, which consists of 2 pacing wires (StreamlineTM, Medtronic, France) implanted surgically in the detrusor muscle (Figure 1) and a platform attached to the head in order to connect the electrode to the stimulator. The electrodes were disposable stainless steel human ventricular pacing wires with a diameter of $0.2 \mathrm{~mm}$. The animals were anesthetized with intraperitoneal injection of urethane. The rat was mounted on the stereotactic apparatus (Stoelting Co, Illinois, USA). A midline incision was made over the skull. The operation area was disinfected with chlorhexidine. A midline laparotomy was made to expose the urinary bladder. Blunt subcutaneous dissection was made through the abdominal incision toward the neck and from there continued toward the cranial incision to make potential spaces. The wires were then tunneled subcutaneously from the scalp toward the abdomen. The pacing wires were driven into the detrusor muscle at the dome and the neck of the bladder and were sutured and secured (Figure 1). The construction was then anchored over the skull with dental cement (Paladur, Heraeus Kulzer GmbH, Germany). A recovery period of two weeks was allowed after surgery and before testing. Electrical stimulation of bladder was performed with a constant current stimulator (World Precision Instruments DS8000) to simulate sensory afferents to PAG. Sensory and motor fibers are more effectively stimulated at longer $(0.4 \mathrm{~ms})$ and shorter $(0.05-0.15 \mathrm{~ms})$ pulse widths respectively[14]. 


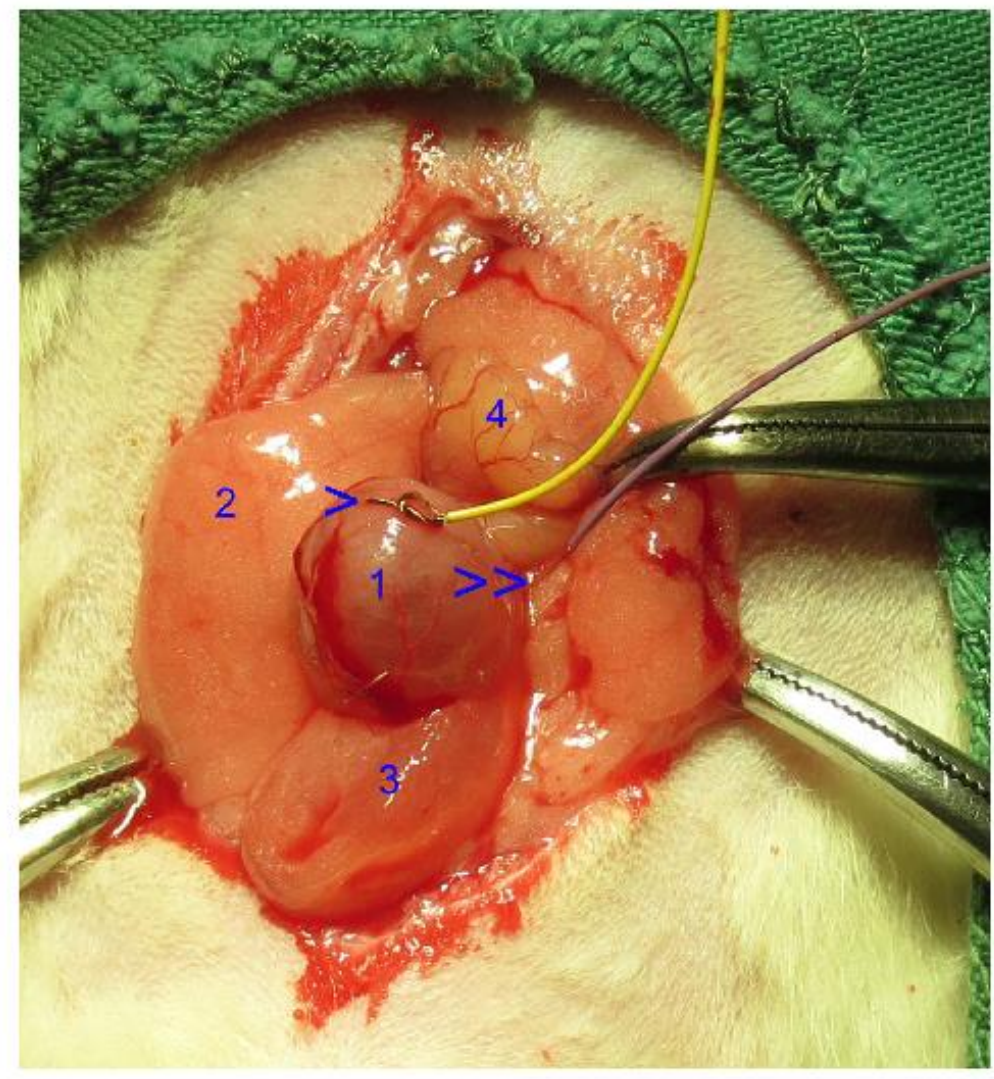

Figure 1

Figure 1. Implantation of stimulation electrodes on the bladder wall; a suprapubic incision is made and the bladder is exposed. Two wires are sutured to the detrusor muscle one at the dome and one at the neck of the bladder ( $>$ and $>>$, respectively).1: bladder, 2: preputial gland, 3: prostate gland, 4: seminal vesicle. Note, since the figure was added during the reviewing process, the photograph is belonging to a follow up experiment, in which an identical electrode implantation was conducted.

After 2 weeks of recovery period, electrical stimulation of the bladder in the stimulation group was conducted giving bipolar square wave pulses of $20 \mathrm{~Hz}$ with an intensity of 2 $\mathrm{mA}$. The subjects underwent continuous stimulation for 1 hour. Sham animals underwent the same surgical procedure with electrode implantation without stimulation being performed. Two hours later all of the animals underwent an overdose intraperitoneal injection of pentobarbital followed by transcardial perfusion-fixation with Somogyi solution. Thereafter the brains were removed, snap frozen and cut in a cryostat (Leica CM3050S) into 30 $\mu \mathrm{m}$ coronal slices. Brains sections were collected into cups to be processed immunohistochemically as free-floating sections. Serial sections were kept at $-80^{\circ} \mathrm{C}$ before staining.

\section{Immunofluorescent staining}

The free floating sections were incubated with rabbit primary antibodies against rabbit derived anti tyrosine hydroxylase (TH, Santacruz, 1/1000) for 48 hours, rabbit anti-5hydroxytriptamine (5-HT, serotonin, 1/50000, courtesy of professor Harry Steinbusch) for 48 hours, vesicular glutamate transporter-1 (VGLUT2 abcam 1/100) for 1 week, anti- 
GAD67 (abcam, 1/100] for 4 days or for sheep derived anti nNOS (1/1000, provided by P. Empson] for 48 hours, all co-labelled with mouse derived anti c-Fos (santacruz $1 / 1000$ ) and thereafter stained with anti-Rabbit alexa 594 and anti-mouse alexa 488 as secondary fluorescent antibodies (1/100, Jackson immuneresearch). Finally all sections were nuclear stained with 1:1000 Hoechst solution in TBS-T and incubated for 10 minutes. Sections were rinsed, mounted on gelatin-coated glasses and cover slipped with $80 \%$ glycerol in tris-buffered saline (TBS).

\section{Confocal microscopic analysis of double stained sections}

The c-Fos positive as well as double stained cells were analyzed using a spinning disk confocal microscope (Olympus, Tokyo, Japan). All stereological investigations were carried out with a stereological computer microscopy system (Stereo Investigator, Microbrightfield Bioscience, Williston, VT, USA). In all sections peripheral borders of the PAG was delineated by visualizing under blue fluorescent spectrum(Hoechst staining), and the borders of ventrolateral column was defined according to a standard stereotaxic rat brain atlas[15]. We then counted the cells within this region stained by appropriate antibodies. In total, six sections per rat containing the vlPAG were delineated. Cell counting was performed in all counting frames of each vlPAG using the optical fractionator probe and $100 \times$ objective lens magnification. Total cell numbers were estimated by established methods, which have been described previously[16].

\section{Statistical analysis}

Data are presented as means and standard errors of means. The quantitative data of the total number of the cells in representative tissue volumes were analyzed using the Independent Samples $T$ test. All statistical analyses were performed with Excel 2013. Pvalues lower than 0.05 were considered significant.

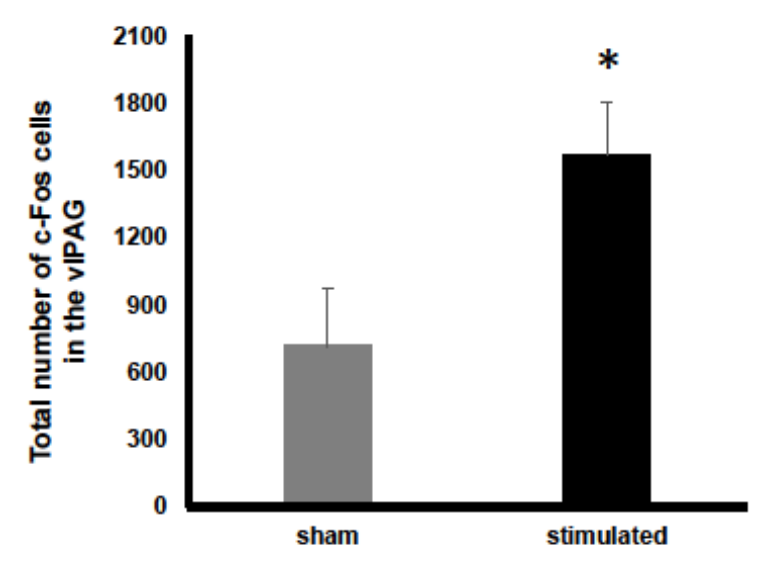

Figure 2

Figure 2. Comparison of c-Fos expression in sham operated and stimulated groups in vlPAG. 


\section{Results}

There was a significantly increased expression of c-Fos in vlPAG of the stimulated group (Figure 2). We performed a design-based stereological analysis of immunohistochemically stained cells expressing TH, 5-HT, VGLUT2, GAD67 and nNOS in the PAG. The total number of cells expressing each individual neurotransmitter was not significantly different between sham and stimulated groups (Figure 3A). The ratio of total number of c-Fos positive cells double labeled with each of above mentioned neurotransmitters to the total c-Fos expressing cells in the appropriate volume of each rat PAG were also compared between the groups (Figure 3B).
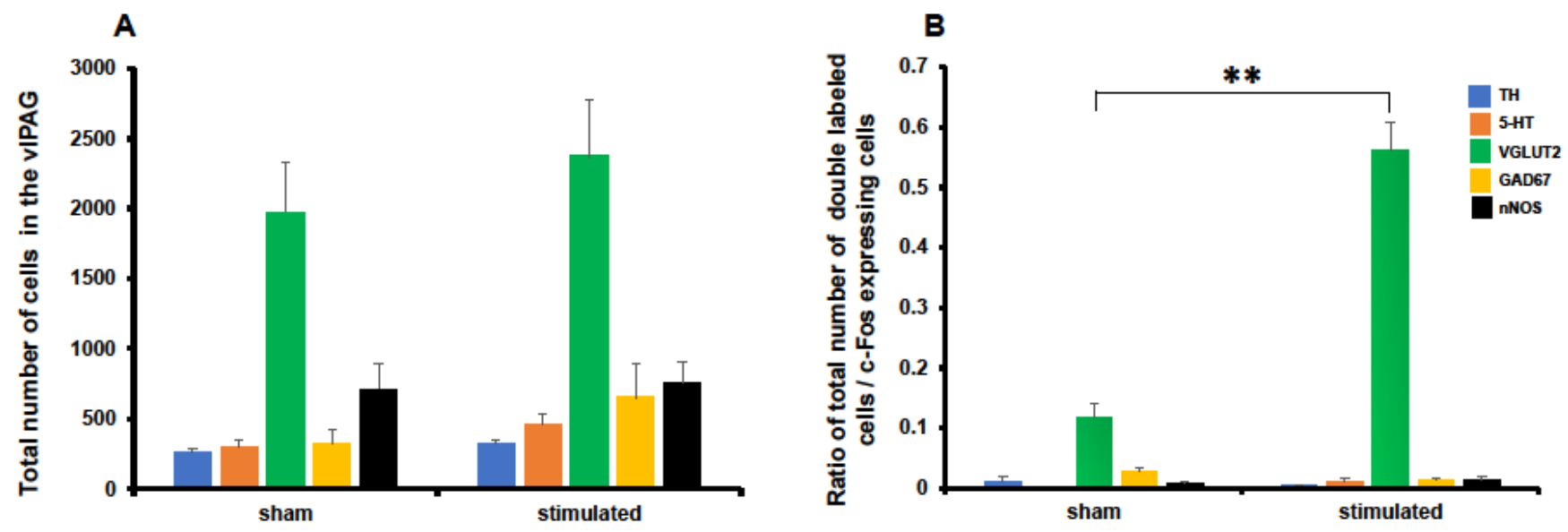

Figure 3. A: Comparison of the total number of cells expressing each marker in the vlPAG between sham and stimulated groups. B: The total number of double labeled cells in the appropriate volume of the brain is divided by the total number of c-Fos expressing cells in each rat for each neurotransmitter studied. Data are presented as SEM; ${ }^{* *} \mathbf{p}<0.001$.

There was no significant difference in the ratio of the number of c-Fos positive cells double labeled with TH, 5-HT, GAD67 and nNOS to the total number of c-Fos expressing cells in this region. Quantitative analysis showed a significant $(\mathrm{p}<0.001)$ increase in ratio of cFos/VGLUT2 positive cells to total c-Fos positive cells in the stimulated versus sham group. This ratio was 0.56 and 0.12 in stimulated and sham groups respectively (Figure 3B). c-Fos+ cells were scattered throughout the vlPAG and double labeled cells were present bilaterally (Figure 4). Representative cytoplasmic and nuclear co-labeling of neurotransmitters and c-Fos are shown in Figure 5. 

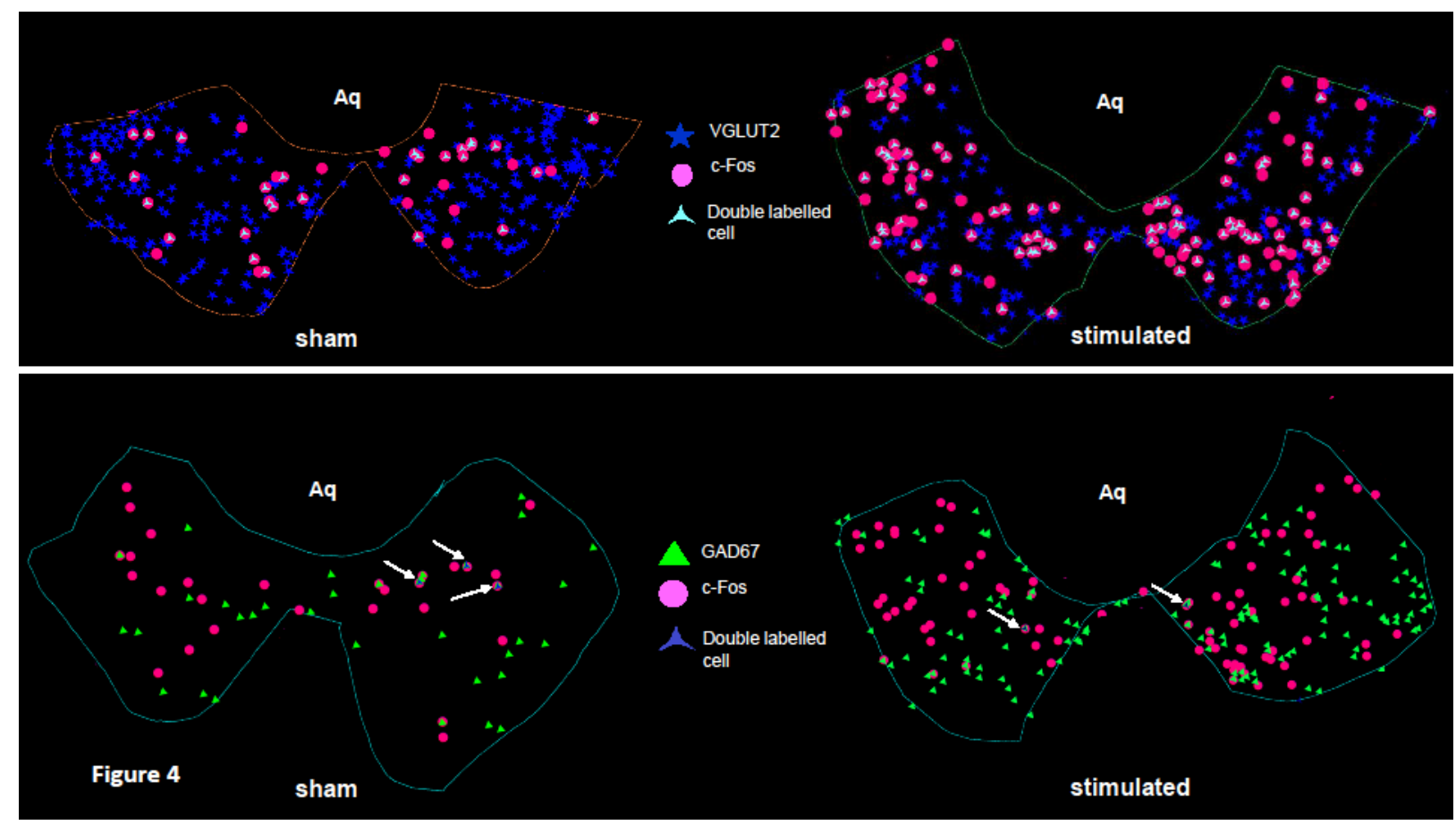

Figure 4. The marker plots figured by the "Stereo Investigator®" software. In each panel the ventrolateral columns of the periaqueductal gray (PAG) are shown with individual cells identified accordingly. In contrast to glutamatergic (VGLUT2) cells there are few GABAergic (GAD67) cells double labelled (arrow) with c-Fos. Aq, Aqueduct.
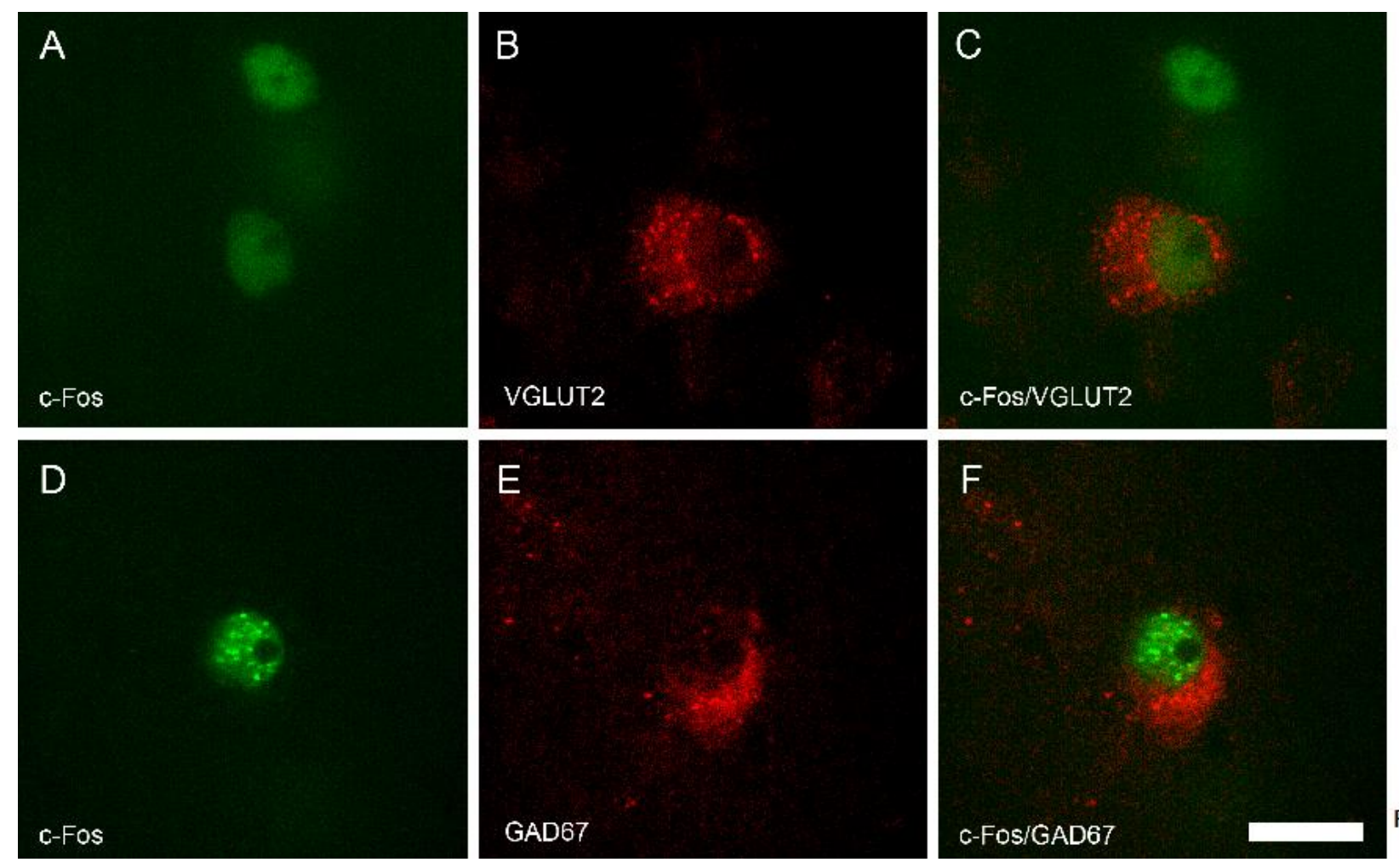

Figure 5

Figure 5. Representative photomicrographs showing c-Fos/VGLUT2 (A-C) and c-Fos/GAD67 (DF) double labeled cells in the periaqueductal gray; scale bar $=10 \mu \mathrm{m}$. 


\section{Discussion}

PAG has immense connections with upstream and downstream structures of the brain structures related to control of micturition. A number of previous studied have revealed vlPAG as the column responsible for sensorimotor communication with bladder[8-13]. We could reproduce these findings in our experimental setting as our data showed a significant increase in c-Fos positive cells in this sub-region following bladder stimulation. Furthermore, our results highlighted the role of glutamatergic cells in relation to bladderPAG circuitry.

The vlPAG has distinct groups of glutamatergic cells. These cells exert various functions, including the control of voiding. Chemogenetic or optogenetic activation of vlPAG glutamatergic and GABAergic neurons stimulated and inhibited the voiding in rats, respectively[17]. The vlPAG GABAergic cells stimulated in this experiment were most probably interneurons[18]. The main inhibitory GABAergic input to the vlPAG relevant to micturition is projected from the dorsolateral PAG (dlPAG)[19]. Glutamatergic cells of the vlPAG also control other important functions, including freezing behavior and analgesia[20]. These two functions are controlled by two separate cell groups in the vlPAG[18]. Whether these cell groups are separate from those vlPAG glutamatergic cells controlling the voiding, or have some overlap remains to be elucidated. Glutamate serves as an excitatory neurotransmitter. The vlPAG receives afferents from the lumbosacral cord[21] and has direct connections with the PMC which then controls micturition by its distant connections with sacral parasympathetic segments. This circuit is especially very active in rodents and probably in the immature human infant while higher decision making brain centers are still underdeveloped. Since vlPAG glutamatergic cells were activated after electrical bladder stimulation in our experiment, these cells probably serve as a bridge in sensorimotor processing, receiving peripheral sensory signals and relaying them to micturition motor control centers of the rat. Glutamatergic vlPAG cells probably stimulate the PMC when receiving suprathreshold sensory signals from a full bladder, to fulfil a micturition reflex. They may project to higher cortical regions in mature human to undergo further analysis and decision making. As a clinical correlate, multiple system atrophy (MSA) is an extrapyramidal disease, which also has micturition symptoms including frequency, urgency, incontinence, or incomplete bladder emptying as its manifestation. The number of glutamatergic cells in the ventrolateral, lateral, and dorsomedial and to a lesser extent dorsolateral PAG columns was shown to be decreased in MSA[22].

On the other hand we did not find any significant involvement of other cell types tested. This may have various reasons, either the micturition pathways would not be dependent on these cell types or the site of their activity is different given our specific experimental setting. Uninfluenced GABAergic transmission in our study may highlight its indirect role over the vlPAG by internal connections via dorsolateral column[23]. One possible reason is projections of dIPAG GABAergic cells to the ventrolateral column to suppress the micturition reflex[19]. Any kind of influence over micturition by dopamine is 
controversial. Microinjection of SCH-23390, a dopamine antagonist, into the PAG had contrary results over micturition in 2 different studies [24, 25]. There is not much known about the micturition related pathways involving the PAG by serotoninergic cells. But the serotoninergic vlPAG is an important regulatory mechanism for the inhibition of ejaculation in rats, and may contribute to SSRI (selective serotonin receptor inhibitor) induced inhibition of ejaculation[26]; although, regulation of the autonomic function of the genital organs is somewhat different from that of the bladder. The bladder's intramural ganglia, dorsal root ganglia and spinal cord, contain NO, which also shows plasticity following pathological lesions such as pelvic nerve injury, chronic bladder irritation and urethral obstruction[27]. There has been no report of a role of nNOS in the brain related to the physiology of the bladder. The negative result we obtained by nNOS may also be related to its regional functional specificity; Most functions mediated by nNOS, including modulation of cardiovascular, behavioral or nociceptive functions, have been found to be mainly active at the dorsal PAG.

Glutamatergic cell population is likely to be the front line of the cascade of neurotransmitters activated in the vIPAG after receiving sensory signals from the bladder. The function of other cell types in the vlPAG may be either indirect via synaptic afferents, or totally non-existent in this specific situation.

\section{References}

1. Barbaresi, P., G. Gazzanelli, and M. Malatesta, Glutamate-positive neurons and terminals in the cat periaqueductal gray matter (PAG): a light and electron microscopic immunocytochemical study. J Comp Neurol, 1997. 383(3): p. 381-96.

2. Clements, J.R., et al., Immunocytochemical localization of serotonin in the rat periaqueductal gray: a quantitative light and electron microscopic study. J Comp Neurol, 1985. 236(1): p. 60-70.

3. Han, F., Y.F. Zhang, and Y.Q. Li, Fos expression in tyrosine hydroxylase-containing neurons in rat brainstem after visceral noxious stimulation: an immunohistochemical study. World J Gastroenterol, 2003. 9(5): p. 1045-50.

4. Chiavegatto, S., C. Scavone, and N.S. Canteras, Nitric oxide synthase activity in the dorsal periaqueductal gray of rats expressing innate fear responses. Neuroreport, 1998. 9(4): p. 571-6.

5. Williams, F.G. and A.J. Beitz, Ultrastructural morphometric analysis of GABAimmunoreactive terminals in the ventrocaudal periaqueductal grey: analysis of the relationship of GABA terminals and the GABAA receptor to periaqueductal grey-raphe magnus projection neurons. J Neurocytol, 1990. 19(5): p. 686-96.

6. Reichling, D.B. and A.I. Basbaum, Contribution of brainstem GABAergic circuitry to descending antinociceptive controls: I. GABA-immunoreactive projection neurons in the periaqueductal gray and nucleus raphe magnus. J Comp Neurol, 1990. 302(2): p. 370-7.

7. Liu, Z., et al., Micturition-related neuronal firing in the periaqueductal gray area in cats. Neuroscience, 2004. 126(4): p. 1075-82.

8. Mitsui, T., et al., Chemical bladder irritation provokes c-fos expression in the midbrain periaqueductal gray matter of the rat. Brain Res, 2003. 967(1-2): p. 81-8. 
9. Duong, M., J.W. Downie, and H.J. Du, Transmission of afferent information from urinary bladder, urethra and perineum to periaqueductal gray of cat. Brain Research, 1999. 819(12): p. 108-119.

10. Taniguchi, N., et al., $A$ study of micturition inducing sites in the periaqueductal gray of the mesencephalon. J Urol, 2002. 168(4 Pt 1): p. 1626-31.

11. Stone, E., J.H. Coote, and T.A. Lovick, Effect of electrical vs. chemical deep brain stimulation at midbrain sites on micturition in anaesthetized rats. Acta Physiologica, 2015. 214(1): p. 135-145.

12. Matsuura, S., G.V. Allen, and J.W. Downie, Volume-evoked micturition reflex is mediated by the ventrolateral periaqueductal gray in anesthetized rats. Am J Physiol, 1998. 275(6 Pt 2): p. R2049-55.

13. Matsuura, S., J.W. Downie, and G.V. Allen, Micturition evoked by glutamate microinjection in the ventrolateral periaqueductal gray is mediated through Barrington's nucleus in the rat. Neuroscience, 2000. 101(4): p. 1053-1061.

14. Byrne, K. and B. Tsui, Pediatric Electrical Nerve Stimulation, in Pediatric Atlas of Ultrasound- and Nerve Stimulation-Guided Regional Anesthesia. 2015, Springer New York. p. 22.

15. Paxinos, G. and C. Watson, The Rat Brain in Stereotaxic Coordinates: Hard Cover Edition. 2007: Elsevier Science.

16. West, M.J., L. Slomianka, and H.J.G. Gundersen, Unbiased Stereological Estimation of the Total Number of Neurons in the Subdivisions of the Rat Hippocampus Using the Optical Fractionator. Anatomical Record, 1991. 231(4): p. 482-497.

17. Verstegen, H., et al., Brain Networks Controlling Bladder Filling and Voiding. Journal of Urology, 2017. 197(4): p. E1150-E1150.

18. Tovote, P., et al., Midbrain circuits for defensive behaviour. Nature, 2016. 534(7606): p. 206-12.

19. Numata, A., et al., Micturition-suppressing region in the periaqueductal gray of the mesencephalon of the cat. American Journal of Physiology-Regulatory Integrative and Comparative Physiology, 2008. 294(6): p. R1996-R2000.

20. Samineni, V.K., et al., Divergent Modulation of Nociception by Glutamatergic and GABAergic Neuronal Subpopulations in the Periaqueductal Gray. eNeuro, 2017. 4(2).

21. Ding, Y.Q., et al., Direct projections from the lumbosacral spinal cord to Barrington's nucleus in the rat: a special reference to micturition reflex. J Comp Neurol, 1997. 389(1): p. 149-60.

22. Benarroch, E.E., et al., Differential involvement of the periaqueductal gray in multiple system atrophy. Auton Neurosci, 2010. 158(1-2): p. 111-7.

23. Jansen, A.S.P., et al., Local connections between the columns of the periaqueductal gray matter: a case for intrinsic neuromodulation (vol 784, pg 329, 1998). Brain Research, 1998. 797(2): p. 368-368.

24. Kitta, T., et al., GABAergic mechanism mediated via D1 receptors in the rat periaqueductal gray participates in the micturition reflex: an in vivo microdialysis study. European Journal of Neuroscience, 2008. 27(12): p. 3216-3225.

25. STONE, E., MIDBRAIN CONTROL OF MICTURITION IN THE RAT chapter 5 phd thesis. 2012: University of Birmingham.

26. Normandin, J.J. and A.Z. Murphy, Serotonergic lesions of the periaqueductal gray, a primary source of serotonin to the nucleus paragigantocellularis, facilitate sexual behavior in male rats. Pharmacology Biochemistry and Behavior, 2011. 98(3): p. 369-375. 
27. Zhou, Y. and E.A. Ling, Neuronal nitric oxide synthase in the neural pathways of the urinary bladder. J Anat, 1999. 194 ( Pt 4): p. 481-96. 


\section{Chapter 6}

Electrophysiological characteristics of neurons in the ventrolateral periaqueductal gray and their correlation with bladder sensory signalling

Aryo Zare, Sandra Schipper, Wolfgang Stein, Yasin Temel, Gommert A. van Koeveringe, Ali Jahanshahi

Conditionally accepted (major revision), Brain Research Bulletin” 


\section{Introduction}

Many of the currently available therapies for urinary incontinence target the peripheral autonomic system, despite many etiologies residing in the central nervous system. Following previous experiments that determined the ventrolateral column of periaqueductal gray matter (vlPAG) to be the main afferent station of bladder sensory signals[12, 29], we aimed for electrophysiological characterization of vlPAG neurons using single unit recording.

\section{Methods}

15 rats were anesthetized and underwent implantation with electrodes at the dome and neck of the bladder, to electrically stimulate the detrusor. After craniotomy, a glass micropipette was inserted in vlPAG to record neuronal action potentials. The detrusor was stimulated by a series of $20 \mathrm{~Hz}$ pulses, for a total duration of 50 seconds at an intensity of $2 \mathrm{~mA}$, to optimize sensory nerve stimulation, for each vlPAG neuron selected. Single unit recordings were performed on a total of 26 neurons. Confirmation of electrode position was made by iontophoretic ejection of Pontamine sky blue.

\section{Results}

The firing rate of vlPAG neurons decreased significantly during the stimulation period. Peristimulus time histogram (PSTH) analysis showed 24 out of 26 neurons to be unresponsive to stimulation. All recorded vlPAG neurons showed irregular firing patterns.

\section{Conclusions}

The change in firing rate may point to an overall inhibitory influence of bladder sensory signals on vlPAG neurons. One hypothesis is the existence of an inhibitory relay station at the vlPAG before sensory bladder signals would affect pontine micturition center. The lack of the inhibitory effect on PSTH may be due to a longer interval between neuronal response and the stimulation.

\section{Keywords}

periaqueductal gray matter, ventrolateral column, urinary bladder, firing pattern 


\section{Introduction}

Even though there is evidence that the central nervous system(CNS) plays a critical role in the causality of urinary incontinence most therapeutic approaches focus on peripheral(nervous) targets (such as the muscarinic acetylcholine receptor)[1]. Several structures in the CNS have been identified to play a role in the regulation of storing urine and voiding. Examples are the pontine micturition centre, parts of the limbic system such as the anterior cingulate gyrus and the parts of the spinal cord such as the sacral nucleus of Onuf. These structures form a complex network, which receive afferent sensory information and provide efferent motor signals to the bladder.

One important mid-brain structures is the periaqueductal gray matter(PAG) involved in sensori-motor regulation of bladder function as well as autonomic [2, 3] and emotional[4] control and nociception[5]. The PAG is composed of separate columns[6, 7]. Various studies mentioned the vlPAG as the column associated with bladder function[8-11]. This has been proven by methods probing both ascending and descending tracts. Noxious stimuli at the level of the bladder increased c-Fos expression in vlPAG in rats [8]. On the other hand, electrical or chemical stimulation of the vIPAG in rats resulted in contraction of the bladder or increased micturition frequency $[9,12]$. Likewise, injection of inhibitory mediators into the vIPAG attenuated bladder contractions and external urethral sphincter electromyographic activity in rats $[11,13]$. PAG activation has also been shown by fMRI studies in both storage and voiding phases of micturition[14]. It can be stated, that there is a reciprocal communication pathway between the brain and the bladder. There is evidence for the efficacy of sacral neuromodulation for the treatment of functional pelvic disorders like voiding dysfunction[15-17].

In order to explore novel targets for neuromodulatory approaches and optimize i.e. stimulation parameters to restore physiology, it is essential to know how brain structures such as the PAG react towards physiological stimuli. For this purpose, we stimulated the bladder with an electrical stimulus set to a threshold not to inititiate the miturition reflex, and evaluated the neurophysiological response of the vlPAG by means of extracellular single unit recordings in anesthetized rats. We hypothesized that vIPAG neurons would react to sensory stimulation by means of a change in firing rate.

\section{Materials and Methods}

\subsection{Surgical preparation}

15 male Sprague rats(350 grams) were anesthetized by intraperitoneal administration of urethane $(7.5 \mathrm{ml} / \mathrm{kg}$ loading dose and $(0.3 \mathrm{ml} /$ repetitive dose) maintenance from a $20 \%$ of weight urethane solution, Sigma Aldrich). They were put on a heating pad set at $37^{\circ} \mathrm{C}$. A midline suprapubic incision was made and the bladder was exposed. Two unipolar ventricular pacing electrodes intended for human use(Medtronic $₫$, Minneapolis, USA, STREAMLINE ${ }^{\mathrm{TM}}$ ) were fixed by means of a suture at the dome and the neck of the bladder(Figure 1). 


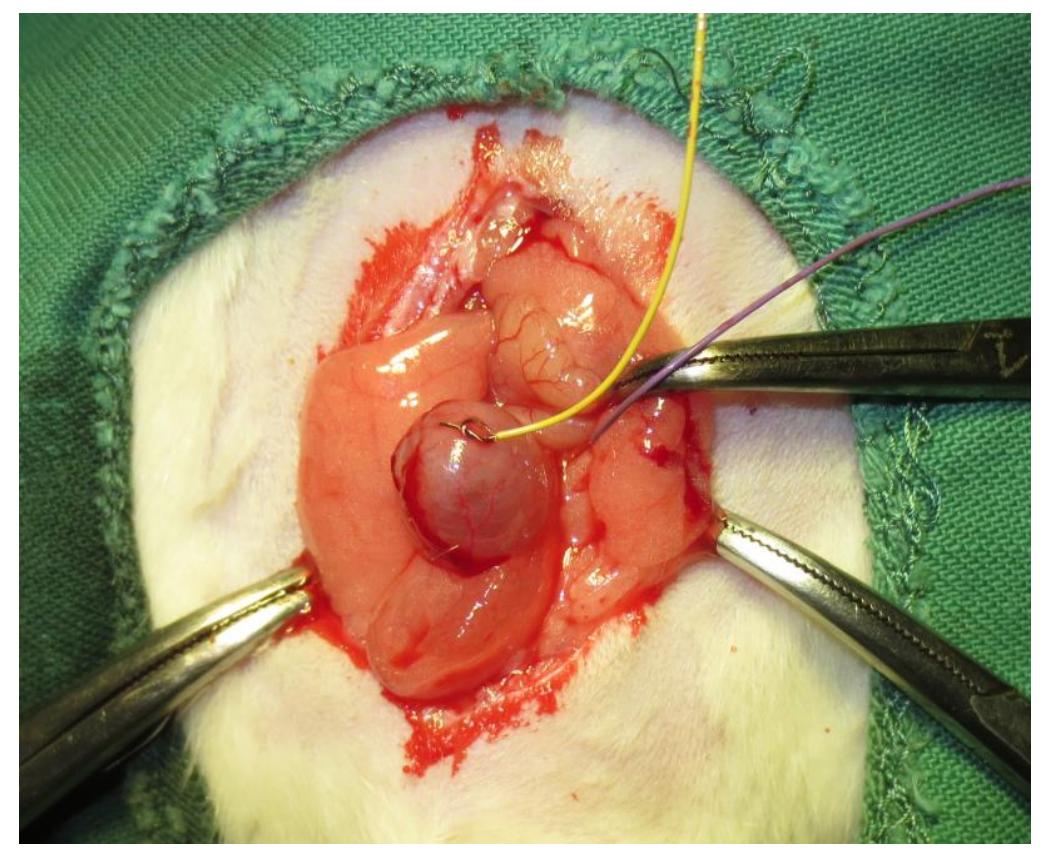

Figure 1. Electrode implantation on rat urinary bladder. One is implanted at dome \& one at neck of bladder to give a bipolar electric field spanning detrusor.

The electrodes were then connected to a stimulator(DS8000, World Precision Instruments) attached to a pulse generator(DLS100, World Precision Instruments, Sarasota, Florida). The rats were then mounted on a standard rat stereotaxic apparatus(Stoelting Co, Illinois, USA). A craniotomy was performed on top of the vlPAG region according to the coordinates relative to Bregma as described in the standard rat brain atlas $(-6.96 \mathrm{~mm}$ upto $-8.16 \mathrm{~mm}$ rostro-caudal relative to Bregma and a maximum of $1.2 \mathrm{~mm}$ mediolaterally and bilateral)[18]. Considerable care was taken to avoid hemorrhage from the sagittal sinus during the durotomy. To achieve this a fine needle tip (30G) was used to lacerate the edges of craniotomy window away from the sinus. Reflexes were frequently checked throughout the experiment and additional urethane was injected if required.

\subsection{Recording}

We used Kwik-Fil ${ }^{\mathrm{TM}}$ filamented borosilicate glass capillaries(outer diameter: $1.5 \mathrm{~mm}$, inner diameter: $0.84 \mathrm{~mm}$, (World precision instruments, Sarasota, Florida ) to record extracellular single units. The glass micropipette was pulled by a pipette puller (Narashige, Japan). Consequently, the pulled tip of the pipet was broken to a diameter of 1 to $2 \mu \mathrm{m}$ under microscopic vision. The micropipette was backfilled with $0.5 \mathrm{M} \mathrm{NaCl}$ solution and inserted into the vIPAG to record extracellular single units. The glass pipet was descended slowly from the cortical surface as a reference point in the ventral direction by means of a hydraulic drum Microdrive(FHC, Bowdoin, ME USA) until the coordinates of the PAG in the dorsoventral direction were reached. Adequate grounding and referencing was implemented in order to reduce (environmental) noise. The micropipette was subsequently connected to an electrophysiology workstation(Alpha Omega Engineering, Nazareth, Israel). Recordings were performed on 26 neurons in 
total in 15 rats within the area of the vlPAG. A baseline recording took place after the neuron had stabilized. Subsequently recording took place until 600 action potentials had been recorded to correct for variance in firing frequency. Then the detrusor was stimulated by a biphasic pulse of $20 \mathrm{~Hz}$ (a pulse period of 50ms) and a pulse width of $0.5 \mathrm{~ms}$ (a duty cycle of 0.01 ) for 50 seconds at an intensity of $2 \mathrm{~mA}$ for each neuron. Neurons, that were lost during this period were excluded from the analysis.

\subsection{Iontophoresis}

After the final recording a iontophoretic injection of 4\% Pontamine sky blue diluted in $0.5 \mathrm{M}$ of $\mathrm{NaCl}(\mathrm{SIGMA}-\mathrm{ALDRICH})$ was performed with the following parameters: direct current, $-20 \mu \mathrm{A}$, with a pulse period of 14 seconds and pulse width of 7 seconds( $50 \%$ duty cycle). The current was set for 15 minutes using the pipette as anode(DS8000 stimulator, A360 isolator, World Precision Instruments, Sarasota, Florida) in order to verify the recording area in all animals. To give bipolar stimulation, the anode consisted of tungsten electrode inserted into the capillary glass filled with Pontamine solution, and the cathode as a stainless steal screw secured on top of the skull(1x2 mm, GHW Modellbau, Germany).

\subsection{Histologic confirmation of electrode location}

Finally, rats were decapitated, the brain was quickly removed and was frozen in $-40^{\circ} \mathrm{C} 2$ methyl-butane(isopentane) for further histochemical confirmation of electrode position. Brains were serially cut by cryostat (Leica CM3050S) in $25 \mu \mathrm{m}$ thick sections and nuclei were visualized by means of fluorescent staining with 1:500 Hoechst solution (blue fluorescence) to facilitate neuroanatomical identification of the PAG within the brain stem. The fluorescent properties of Pontamine Sky blue, making it appear red, were used to obtain pictures under the microscope in the blue (for background Hoechst staining) and red (for Pontamine) filter emission spectra, which were consequentially merged and the injection site was confirmed.

\subsection{Analysis}

The analysis was performed in Spike $2{ }^{\circledR}$ (Cambridge electronic design, Cambridge, ENGLAND), SPSS version 23 and MicroSoft $\AA$ Excel ${ }^{\circledR} 2016$. Average neuronal firing rate was calculated during two minutes before the start of stimulation(consequently called baseline), during stimulation (called stimulation) and during three consecutive periods lasting one minute each after the end of stimulation (called post-stimulation). We have chosen to use these time periods in order to differentiate the immediate effect during stimulation, and the immediate, intermediate and long-term result after stimulation. Furthermore, peristimulus time histograms(PSTH) during the stimulation period were made for each individual neuron in order to evaluate the direct response of the neuron during stimulation. For this purpose the optimal bin size was calculated as published before[19] and getting assistance from a Java web application[20]. This optimization resulted two bins during the $50 \mathrm{~ms}$ stimulation pulse period for all vlPAG recorded units. Tailored to our specific settings and corresponding to previous literature[21], we defined a responsive unit as showing at least $70 \%$ change in average firing rate comparing the 
average frequency before and after the stimulus. Thus, excitation is defined as average firing rate at the second bin to be less than $70 \%$ of the first bin and inhibition as average firing rate of the first bin to be less than $70 \%$ of the second bin. In order to evaluate the firing properties and more specifically the regularity of the recorded neurons interspike intervals(ISI) were calculated. ISI coefficient of variation(CoV) defined as standard deviation of ISI divided by average ISI for each neuron, was measured as an index of regularity of firing of neuron[22]. Moreover, for the characterization of neuronal populations a waveform average was made from all individual neurons during baseline firing. In the average waveform, the latency between the peak and the trough and the ratio of peak to trough amplitude was calculated for each neuron separately. The fraction response of the neuronal firing during stimulation relative to the period before stimulation was calculated as follows: $\left(\frac{\text { mean firing rate during stimulation }}{\text { mean firing rate before stimulation }}-1\right)$. This parameter was then used for regression analysis relative to waveform characteristics.

\section{Results}

Neurons in the vlPAG fire with an average frequency of $4.5 \mathrm{~Hz} \pm 4.7$. Their frequency ranged from 0.1 to $17.9 \mathrm{~Hz}$. The relative firing rate of vlPAG neurons calculated as the fraction change of the firing rate relative to period before stimulation significantly decreased during the stimulation period with a mean of 0.83(Figure 2). To perform a repeated measures ANOVA, we first checked for normality and then conducted a Mauchly's test, which indicated a violation of the assumption of sphericity $(p=0.000)$. Hence we performed a multivariate analysis of variance (MANOVA) which resulted $\mathrm{F}(4,22)=2.976, \mathrm{p}=0.042$, Wilk's Lambda $=0.649$ (Figure 2). Follow-up test with Bonferroni correction resulted a $\mathrm{p}=0.03$ regarding the periods before and during the stimulation. Two by two follow-up comparison of other time points did not show any significance. 


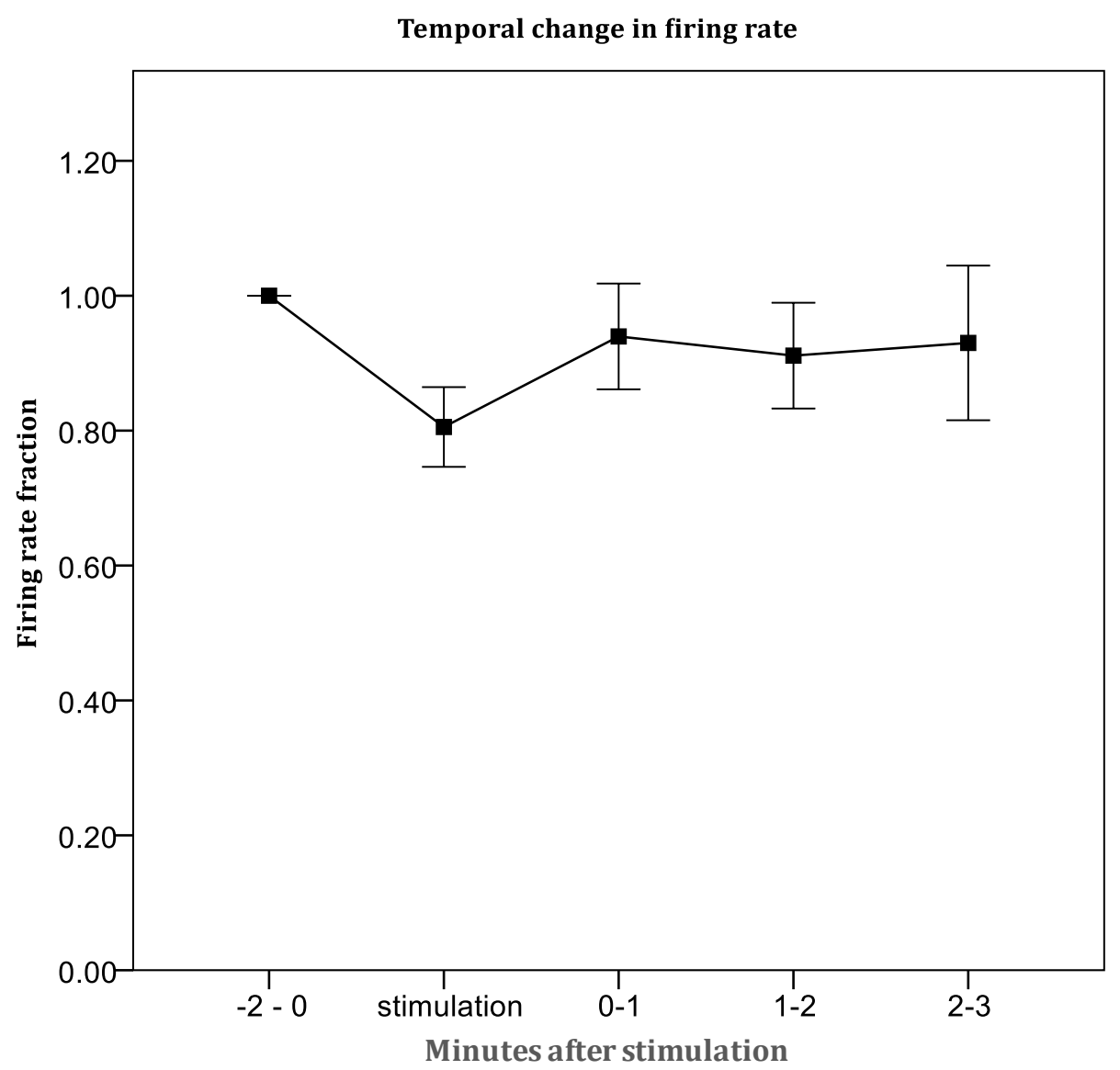

Figure 2. Comparison of firing rate of neurons in time span. The firing rate has been expressed as fraction relative to the period before start of stimulation, considered as baseline. Error bars are S.E.M.

PSTH analysis showed no response in 24 out of 26 neurons. One neuron showed inhibitory and one excitatory response according to the defined criteria(Figure 3).

Histograms of waveform characteristics of recorded neurons show a more normal distribution in peak/trough ratio relative to peak-trough latency(Figures 4 and 5).

We checked possible correlation of waveform characteristics and response to stimulation regarding firing rate by regression analysis(Figure 6). Linear regression revealed no meaningful correlation between waveform characteristics including peak-trough latency and peak/trough amplitude ratio and firing rate response $\left(R^{2}=0.16\right)$ (Figure 6).

ISI coefficient of variation(CoV) of the recorded neurons ranged from 0.15 to 2.0 with a mean of 0.99 (Figure 7). The position of the electrodes were confirmed by microscopic observation of the Pontamine dot (figure 8). 

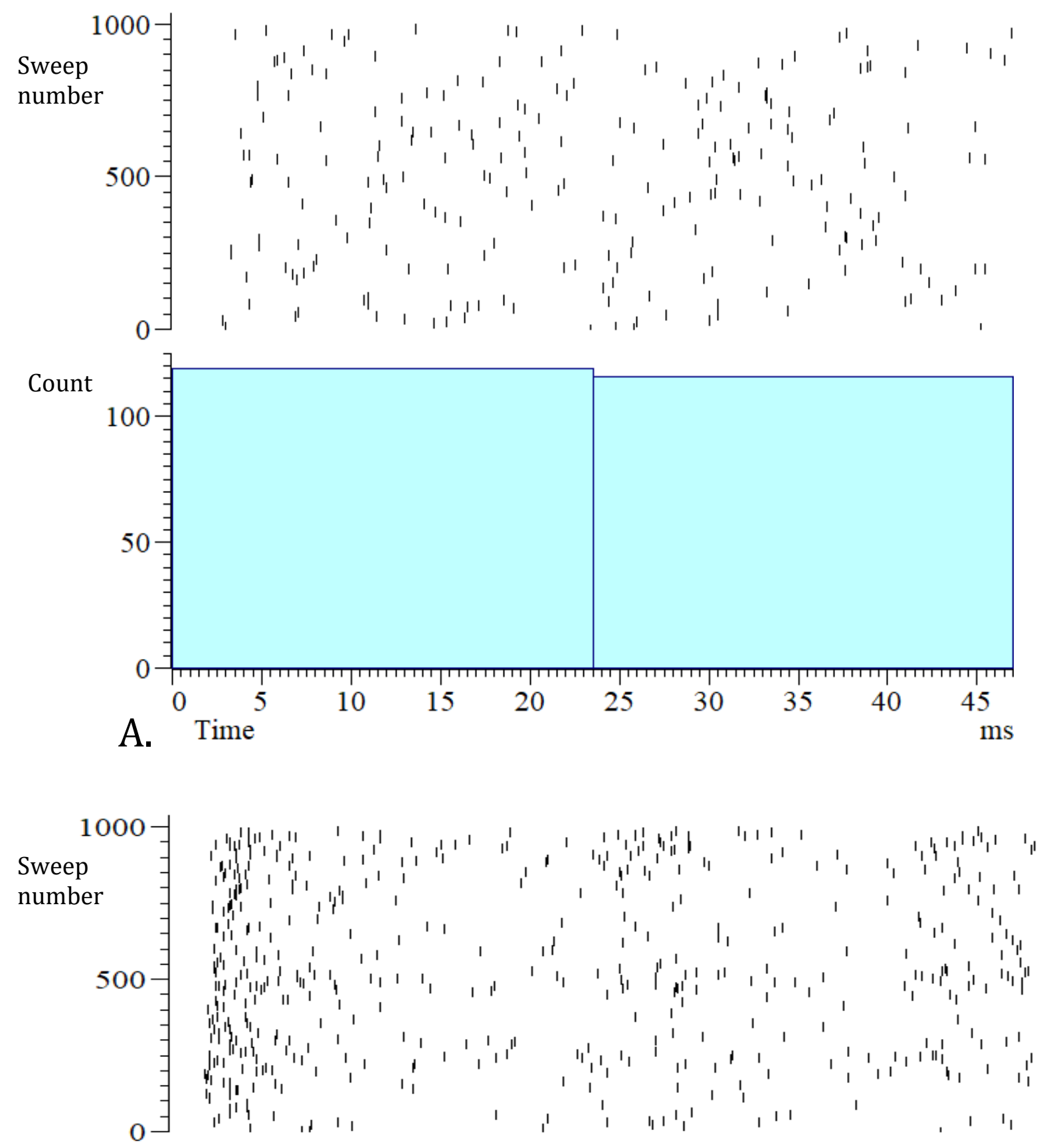

Count

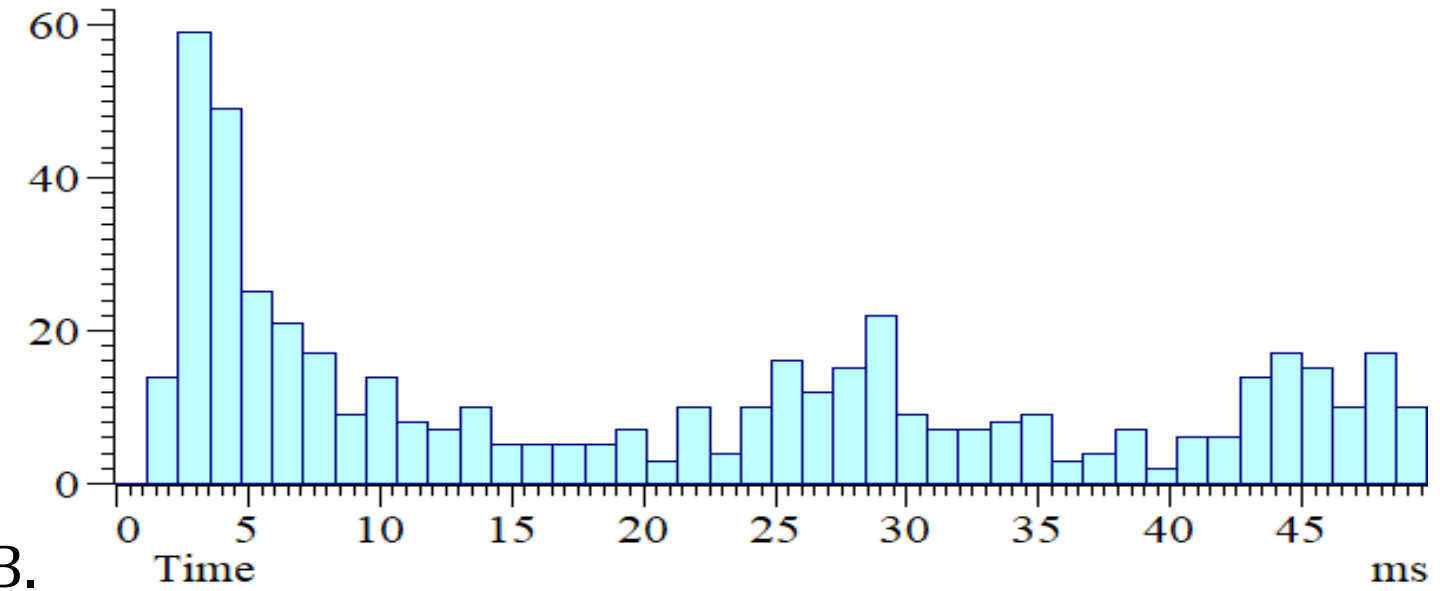

Figure 3. Peristimulus time histogram (PSTH) 
A. A sample PSTH of a vIPAG recorded unit. Note that the number of bins were standardized to 2 (details described in the main text), which takes into account the firing rate, regularity $\&$ total number of spikes.

B. An excited neuron recorded in lateral PAG with the same experimental setup. Optimal bin size was calculated by the same method as in $A$.

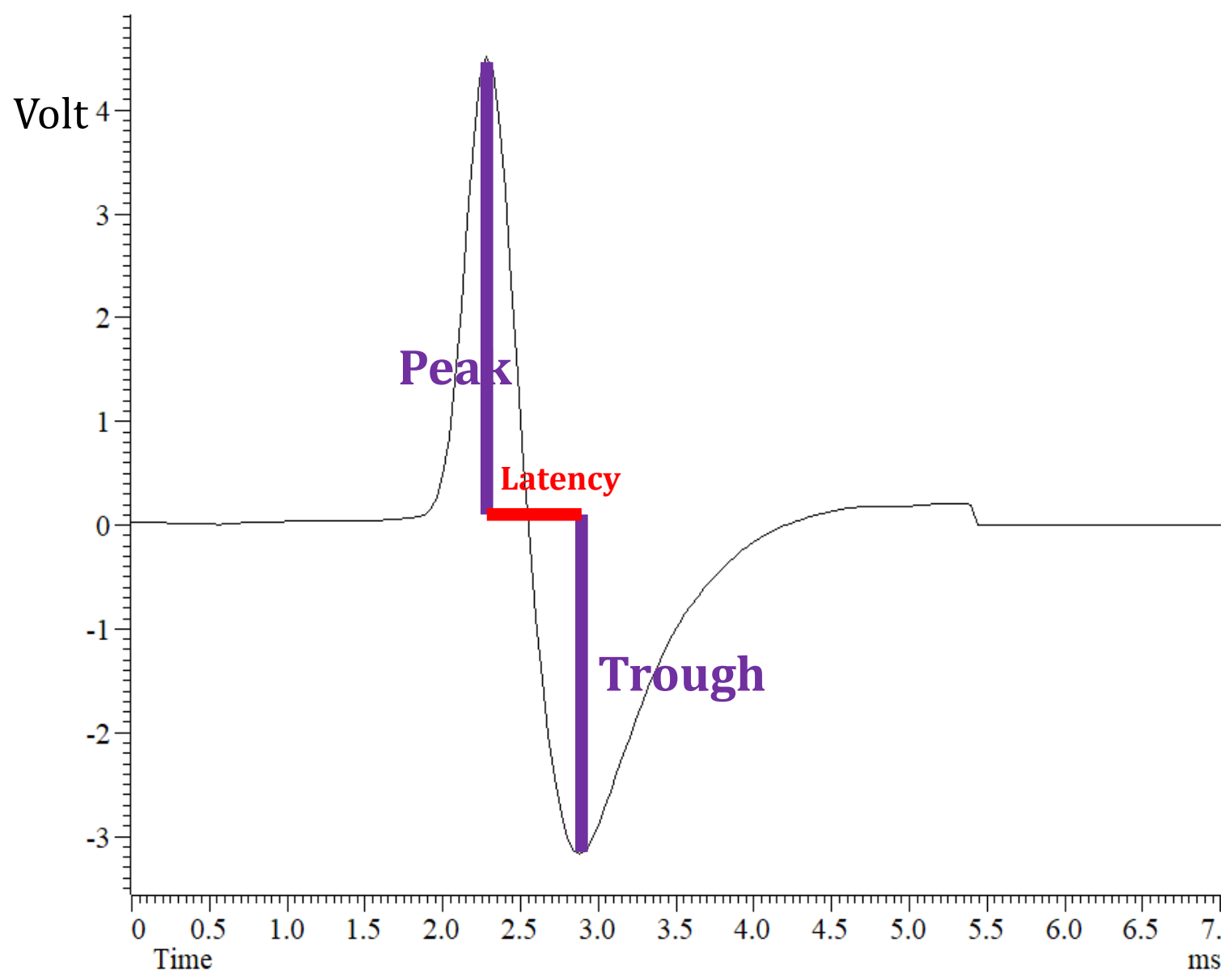

Figure 4. Average waveform of one individual recorded neuron showing parameters used for waveform characterization. 


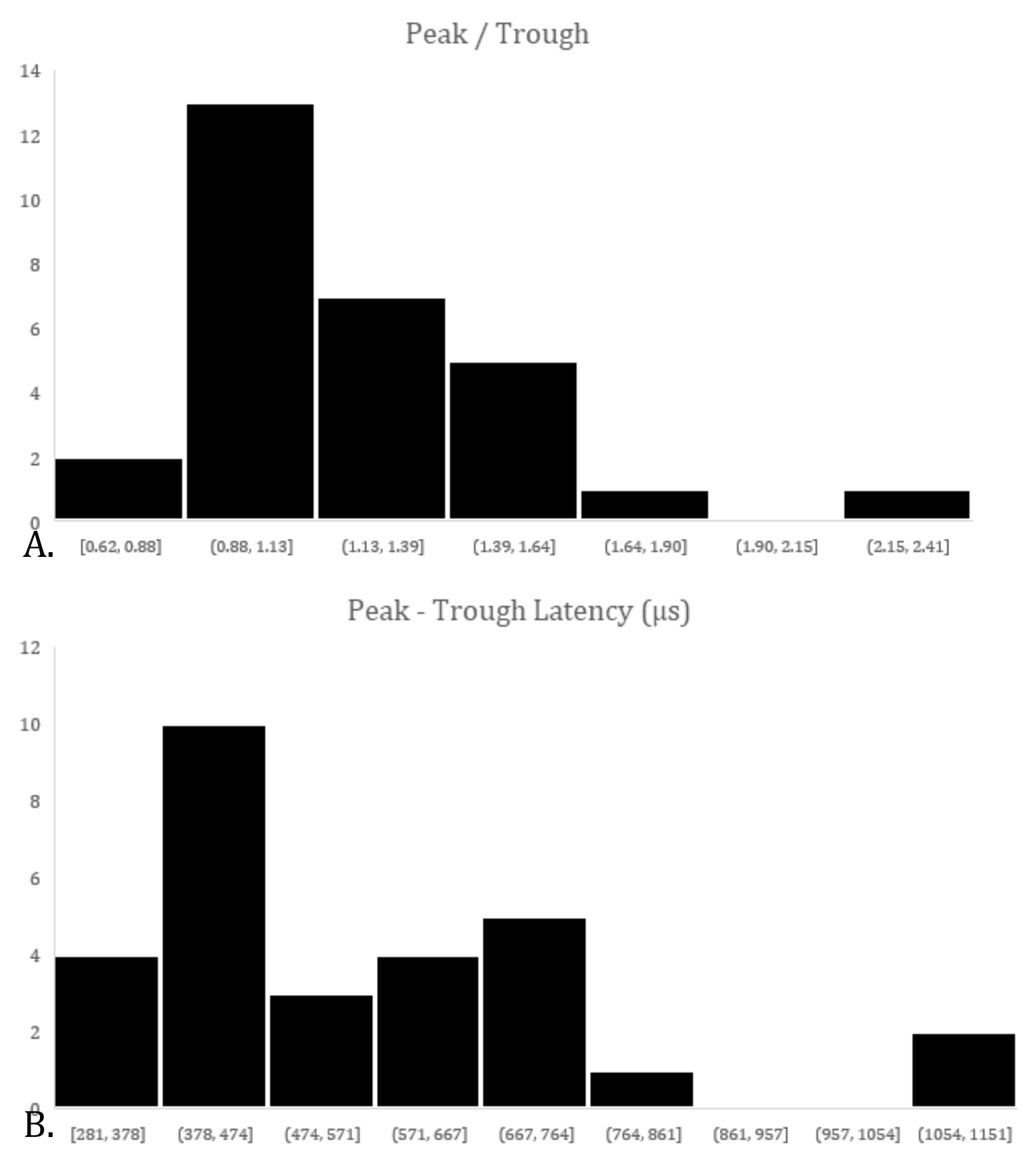

Figure 5. Waveform characteristics of recorded vlPAG neurons, all measured in the period before stimulation. Histograms covering ratio of peak to trough(A) and peak to trough latency(B), including 26 recorded units. 


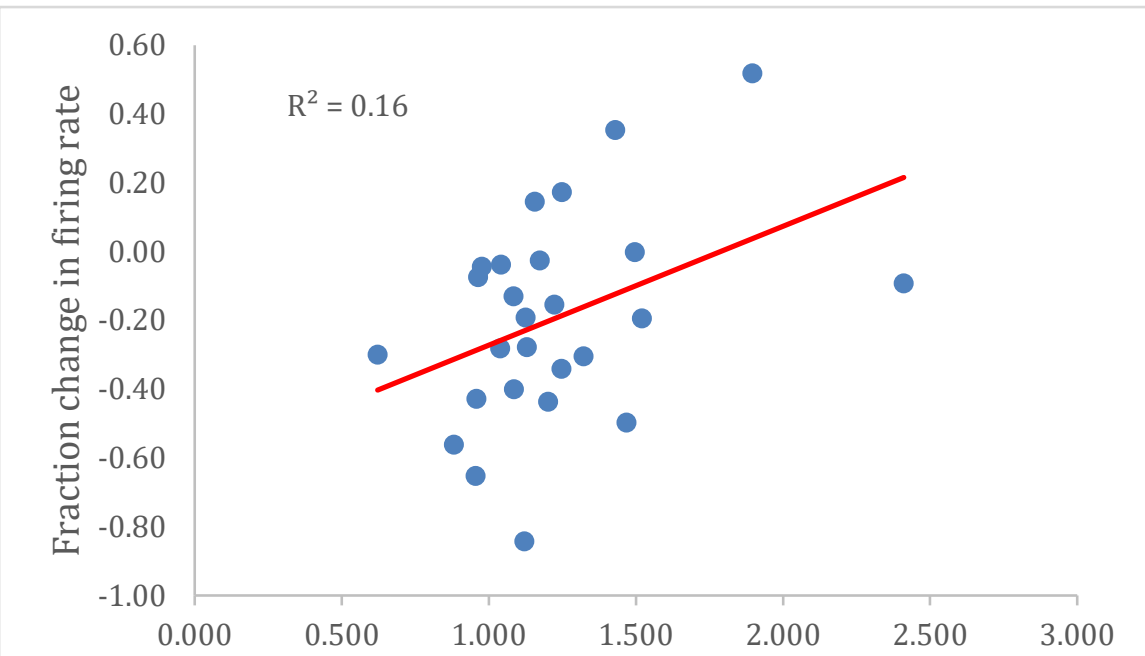

A.

peak/trough ratio
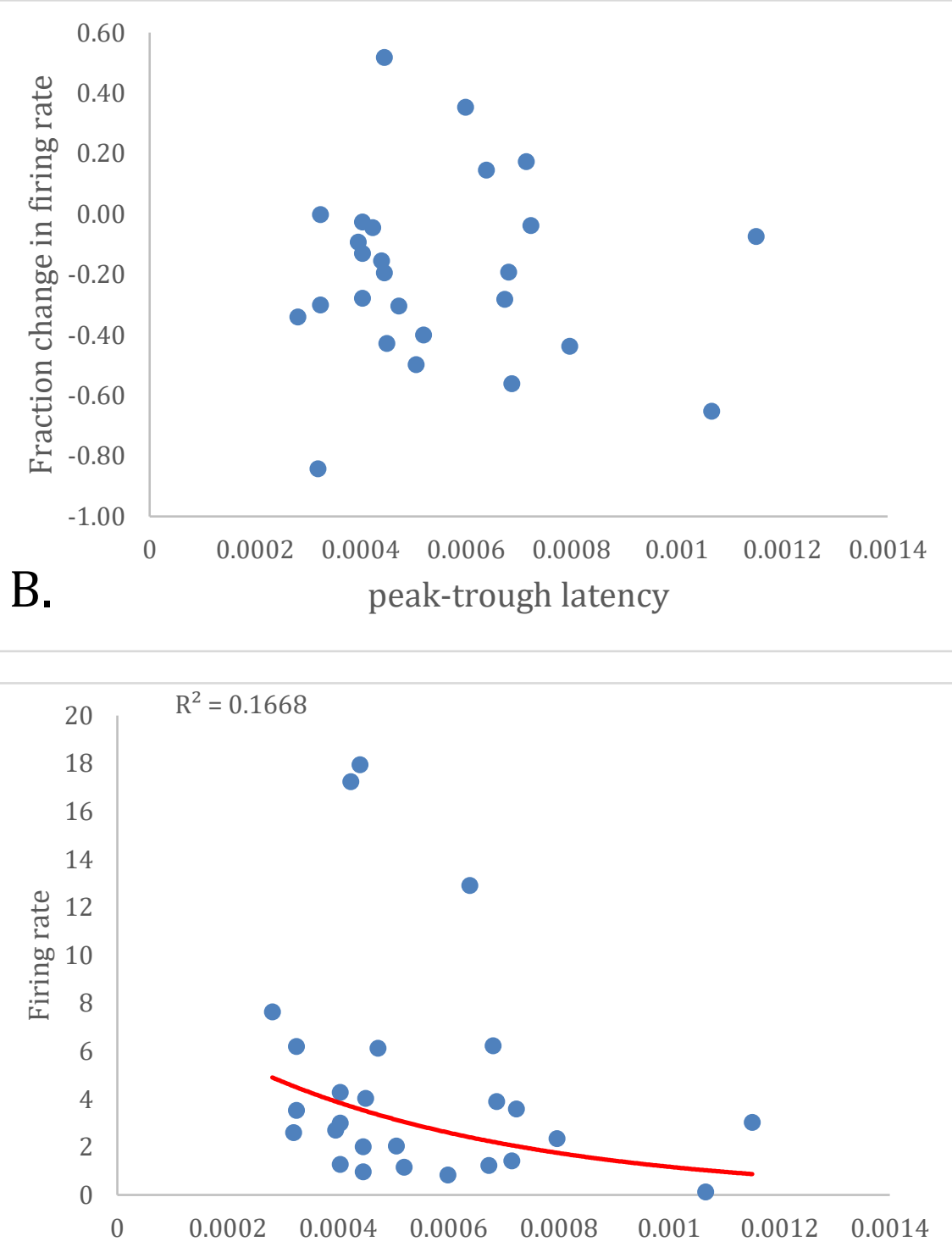

C.

peak-trough latency 
Figure 6. Regression analysis of fraction change in firing rate as response variable \& waveform characteristics as predictors. As evident one can not predict change in firing rate based on waveform characteristics.

A. Fraction change in firing rate vs peak/trough ratio.

B. Fraction change in firing rate vs peak-trough latency

C. The basal firing rate of the neurons are also not related to their waveform characteristics.

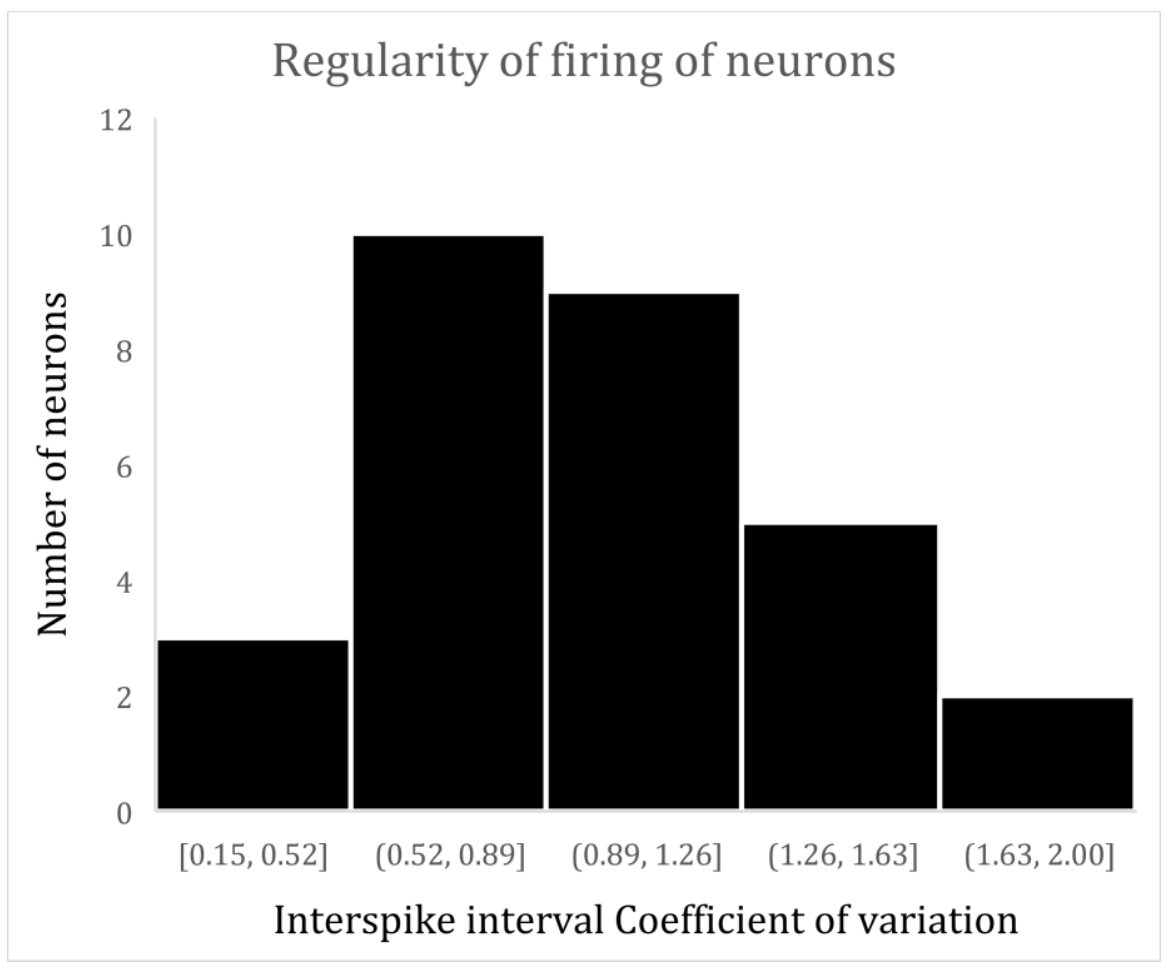

Figure 7. Average interspike intervals coefficient of variation(CoV) recorded from 26 neurons within vlPAG. 

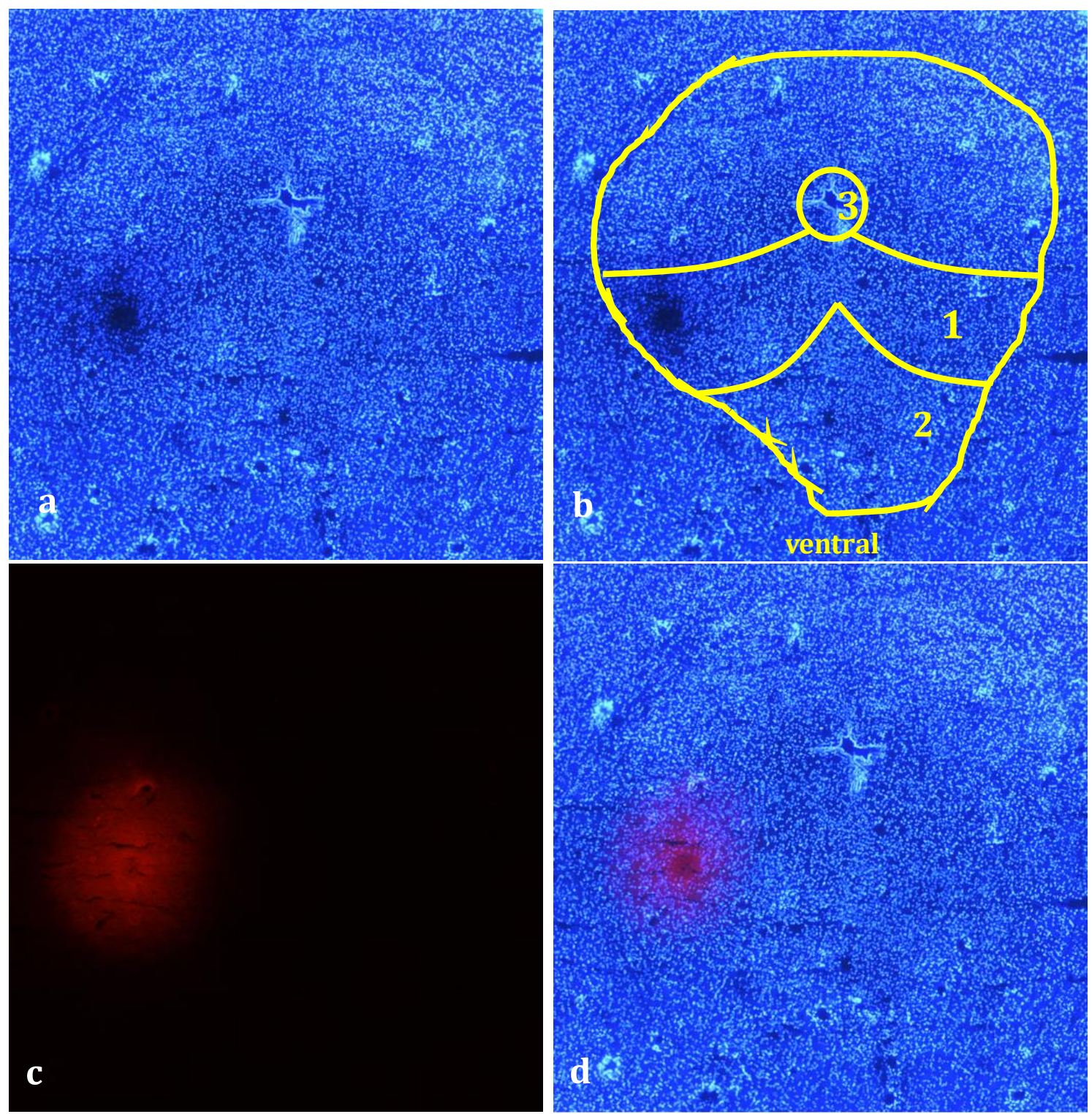

Figure 8. Iontophoresis. Transverse sections of PAG stained with Hoechst(observed in blue spectrum)(a), to clarify the borders of PAG. The individual columns are separated according to a standard stereotactic atlas(b). Pontamine sky blue can be observed in red spectrum(c) and merged to give a composite image(d).

b1: vIPAG area

b2: area of dorsal raphe $\&$ cranial nerve nuclei.

b3: aqueduct 


\section{Discussion}

In this work we aimed for obtaining a clearer view of the role of the vlPAG regarding the physiology of the micturition. The stimulation parameters we used were based on previous experiences which lead to bladder contraction without inducing micturition. Sensory peripheral nerve fibers are more effectively stimulated at longer(0.4 ms) and motor fibers at shorter(0.05-0.15 ms) pulse widths[23]. Electrophysiologic characteristics of PAG neurons were described in a number of papers considering micturition or noxious stimuli[24, 25], but none of them were focusing on the vlPAG alone. Previous studies with single unit recordings in PAG showed different neuronal firing patterns during the micturition reflex within ventrolateral and lateral columns, some increasing, some decreasing and some unresponsive during micturition[26]. The diverse responses of neurons may reflect different neuronal types corresponding to different functions like detrusor contraction, sphincter relaxation or neurons with no micturition related effect. The average decrease in firing rate during the stimulation period found in our study indicates an overall inhibitory influence of the bladder sensory signals on vlPAG neurons. However this does not exclude possible excitatory influences since we only compared the population response. We could not differentiate the various neuronal types electrophysiologically to perform a separate analysis for each group. PSTH analysis denied any instant excitatory or inhibitory effect of the stimulation pulse on the neuronal firing. So at least the decrease in firing rate during the stimulation period could not be explained by connections which would involve less than $50 \mathrm{~ms}$ (stimulation pulse period) of signal propagation time lapse between the stimulation and the recording sites.

Technical reasons may also underlie lack of responsive effects evident on PSTH. High frequency $(20 \mathrm{~Hz})$ and short period(50 seconds) of stimulation which were optimized for bladder sensory fibers, may mask at least some responsive vlPAG neurons. To exclude nociceptive signals and anxiety secondary to these stimulation parameters, behavioral tests were done(our own work, unpublished). Different neuronal types have different electrophysiologic characteristics. The distribution of some waveform characteristics is shown in figure 5 which were tested for being predictive of the neuronal responses to the stimulation(figure 6). The negative result may be partially due to the low number of total neurons we used for the analysis.

The regularity of the firing of the neurons differs in various neuronal types and in different brain regions. There are various ways to quantitate spike train variability[27]. One simple parameter is $\mathrm{CoV}$ shown in Figure 7. Arbitrarily a $\mathrm{CoV}$ of $>0.1$ is considered an irregular firing pattern, which is the case in all of these vlPAG recorded units[28]. Comparing the regularity of the firing rate in the vlPAG with the other PAG columns, would help to gain more insight to the electrophysiologic differences between various PAG columns.

In parallel we described a simpler method of microscopic potamine observation. Fluorescent properties of pontamine have been already used for counterstaining in immunofluorescence[29], but to our knowledge there was no report of using its 
fluorescent properties for localization of electrode position. As pontamine has a bright red fluorescence, traces of it can be easily detected by fluorescent microscopy. This would be particularly helpful when the localization needs to be in a tiny restricted area. Heavier dye injection into the region to make its detection easier by light microscopy would result in progressive dispersion of the dye to a more distant periphery of the pipette tip, decreasing the precision of the localization. Double staining with Hoechst and visualization in the blue spectrum gives a scheme of background anatomical location which can be merged with pontamine visible in the red spectrum. This may be a better alternative to light microscopy, with a more precise localization without the need to inject so much dye into the region (Figure 8).

\subsection{Conclusions}

The decrease in the firing rate may be due to an overall inhibitory effect of the peripheral stimulation on vlPAG neurons. One hypothesis is inhibition of the vlPAG by sensory signals emerging from a suprathreshold filled bladder. This may have a secondary effect on other central micturition circuitry. The vlPAG neurons have diverse electrophysiological characteristics.

\section{References}

1. Emily S Lukacz, M., MAS, Treatment of urgency incontinence/overactive bladder in women, M. Linda Brubaker, FACOGKenneth E Schmader, MD, Editor. 2018, UpToDate (Accessed on 2018-2-5).

2. Rathner, J.A. and S.F. Morrison, Rostral ventromedial periaqueductal gray: a source of inhibition of the sympathetic outflow to brown adipose tissue. Brain Res, 2006. 1077(1): p. 99-107.

3. Farkas, E., A.S. Jansen, and A.D. Loewy, Periaqueductal gray matter input to cardiac-related sympathetic premotor neurons. Brain Res, 1998. 792(2): p. 179-92.

4. Mouton, L.J. and G. Holstege, The Periaqueductal Gray in the Cat Projects to Lamina-Viii and the Medial Part of Lamina-Vii Throughout the Length of the Spinal-Cord. Experimental Brain Research, 1994. 101(2): p. 253-264.

5. Budai, D., I. Harasawa, and H.L. Fields, Midbrain periaqueductal gray (PAG) inhibits nociceptive inputs to sacral dorsal horn nociceptive neurons through alpha(2)-adrenergic receptors. Journal of Neurophysiology, 1998. 80(5): p. 2244-2254.

6. Bandler, R. and M.T. Shipley, Columnar organization in the midbrain periaqueductal gray: modules for emotional expression? Trends Neurosci, 1994. 17(9): p. 379-89. 
7. Parvizi, J., G.W. Van Hoesen, and A. Damasio, Selective pathological changes of the periaqueductal gray matter in Alzheimer's disease. Annals of Neurology, 2000. 48(3): p. 344-353.

8. Mitsui, T., et al., Chemical bladder irritation provokes c-fos expression in the midbrain periaqueductal gray matter of the rat. Brain Res, 2003. 967(1-2): p. 81-8.

9. Taniguchi, N., et al., A study of micturition inducing sites in the periaqueductal gray of the mesencephalon. J Urol, 2002. 168(4 Pt 1): p. 1626-31.

10. Duong, M., J.W. Downie, and H.J. Du, Transmission of afferent information from urinary bladder, urethra and perineum to periaqueductal gray of cat. Brain Research, 1999. 819(12): p. 108-119.

11. Matsuura, S., G.V. Allen, and J.W. Downie, Volume-evoked micturition reflex is mediated by the ventrolateral periaqueductal gray in anesthetized rats. Am J Physiol, 1998. 275(6 Pt 2): p. R2049-55.

12. Stone, E., J.H. Coote, and T.A. Lovick, Effect of electrical vs. chemical deep brain stimulation at midbrain sites on micturition in anaesthetized rats. Acta Physiologica, 2015. 214(1): p. 135-145.

13. Matsuura, S., J.W. Downie, and G.V. Allen, Micturition evoked by glutamate microinjection in the ventrolateral periaqueductal gray is mediated through Barrington's nucleus in the rat. Neuroscience, 2000. 101(4): p. 1053-1061.

14. Tai, C.F., et al., Brain Switch for Reflex Micturition Control Detected by fMRI in Rats. Journal of Neurophysiology, 2009. 102(5): p. 2719-2730.

15. Sukhu, T., M.J. Kennelly, and R. Kurpad, Sacral neuromodulation in overactive bladder: a review and current perspectives. Res Rep Urol, 2016. 8: p. 193-199.

16. Rosen, A., et al., Effects of Sacral Neuromodulation on Urinary and Fecal Incontinence. Isr Med Assoc J, 2015. 17(6): p. 351-5.

17. Pettit, P.D., J.R. Thompson, and A.H. Chen, Sacral neuromodulation: new applications in the treatment offemale pelvic floor dysfunction. Curr Opin Obstet Gynecol, 2002. 14(5): p. 5215.

18. Paxinos, G. and C. Watson, The Rat Brain in Stereotaxic Coordinates: Hard Cover Edition. 2007: Elsevier Science.

19. Shimazaki, H. and S. Shinomoto, A method for selecting the bin size of a time histogram. Neural Comput, 2007. 19(6): p. 1503-27.

20. Shimazaki, H. Selecting the Bin Size of a Time Histogram. [JAVA application] 2010; version 2.4:[Available from: http://www.ton.scphys.kyotou.ac.jp/ shino/toolbox/sshist/hist.html

21. Beyeler, A., et al., Stimulation of serotonin2C receptors elicits abnormal oral movements by acting on pathways other than the sensorimotor one in the rat basal ganglia. Neuroscience, 2010. 169(1): p. 158-70.

22. Holt, G.R., et al., Comparison of discharge variability in vitro and in vivo in cat visual cortex neurons. J Neurophysiol, 1996. 75(5): p. 1806-14.

23. Byrne, K. and B. Tsui, Pediatric Electrical Nerve Stimulation, in Pediatric Atlas of Ultrasound-and Nerve Stimulation-Guided Regional Anesthesia. 2015, Springer New York. p. 22.

24. Crook, J. and T. Lovick, Urodynamic function during sleep-like brain states in urethane anesthetized rats. Neuroscience, 2016. 313: p. 73-82.

25. Sharma, R., et al., Neuronal responses of periaqueductal gray to peripheral noxious stimulation. Indian J Physiol Pharmacol, 1999. 43(4): p. 449-57. 
26. MATSUMOTO Kengo, S.T., YAMAMOTO Gen Characterization of micturition-related single neuron activities and their distribution in periaqueductal gray matter (PAG) in anesthetized rats. J Pharmacol Sci 2006. 100: p. 251.

27. Kuebler, E.S., Thivierge, J.P. , Spiking variability: Theory, measures and implementation in matlab The Quantitative Methods for Psychology, 2014. 10(2): p. 131-142.

28. Spike train analysis workshop. 2015; RM 07.08.2015 School of Life Sciences UNMS, prerelease STA v0.991 [Available from: www.nottingham.ac.uk/neuronalnetworks/STAnalysis\%20Workshop\%20v0.991.doc

29. Cowen, T., A.J. Haven, and G. Burnstock, Pontamine sky blue: a counterstain for background autofluorescence in fluorescence and immunofluorescence histochemistry. Histochemistry, 1985. 82(3): p. 205-8. 


\section{Chapter 7}

\section{Local Regulatory Changes in Isolated Bladders of Alzheimer Mice}

Aryo Zare*, Ramona Hohnen*, Mohammad Sajjad Rahnama'i, Devon Barnes, Celine Meriaux, Gommert A. van Koeveringe

* These authors contributed equally to the work. 


\section{Abstract}

Objectives

To shed light on mechanism of incontinence in Alzheimer's disease we performed this exvivo study on bladders of an Alzheimer's disease mouse model.

\section{Methods}

Bladders of 12 transgenic Alzheimer's disease mice of the APPSL/PS1 ${ }^{\mathrm{M} 146 \mathrm{~L}}$ model and 7 wild type mice were dissected, catheterized transurethrally and placed in an organ bath. The bladders were stimulated with $1 \mu \mathrm{M}$ of carbachol and then with $0.1 \mathrm{M} \mathrm{KCl}$. The area under the curve was analysed from the beginning of the contraction until the maximum intravesical pressure after addition of either carbachol or $\mathrm{KCl}$. Immunohistochemical staining was performed on bladders using a primary antibody against amyloid precursor protein and $\beta$-amyloid.

\section{Results}

A lower frequency of asynchronized microcontractions was recorded during the baseline state in bladders from Alzheimer's disease mice. The area under the curve was lower in bladders from Alzheimer's disease mice compared to wild type mice after $\mathrm{KCl}$ administration. In contrast to wild type mice, immunoreactivity against Alzheimer's disease markers was detected in bladders of Alzheimer's disease mice.

\section{Conclusion}

We found structural and functional alterations in this Alzheimer's disease model. As we used dissected bladders in an organ bath setting, direct pathophysiological involvement of the bladder tissue itself is likely in this model.

\section{Highlights}

- Trangenic AD mice bladders are functionally different from the normal mice.

- Trangenic AD mice bladders express $\beta$-amyloid.

- $\mathrm{AD}$ induced voiding dysfunction may have peripheral causes.

\section{Key Words :}

Age, Alzheimer's disease, Bladder, Incontinence, Lower urinary tract symptoms

Abbreviations and Acronyms

Overactive bladder $(\mathrm{OAB})$

Alzheimer's disease (AD) 
Wild type (WT)

Area under the curve (AUC)

Amyloid precursor protein (APP)

Endoplasmic reticulum (ER)

Rho-associated protein kinase (ROCK)

Tris-buffered saline (TBS)

Tris-buffered saline containing Triton X (TBST)

\section{Introduction}

Incontinence is more common among the elderly and among women, affecting about $30 \%$ of elderly women and $15 \%$ of elderly men[1]. Furthermore, neurodegenerative disorders or cognitive decline are common causes of incontinence in the elderly population. Urinary incontinence and severe cognitive decline are associated with Alzheimer's disease(AD) and incontinence usually precedes severe mental failure in diffuse Lewy body disease[2]. Treatment of both conditions, dementia and incontinence, is challenging. Cholinesterase inhibitors as rivastigmine are used as a treatment for AD dementia[3], whereas anticholinergic drugs such as tolterodine and solifenacine are commonly used to treat $\mathrm{OAB}$. Amyloid- $\beta$, which has been shown to accumulate in $\mathrm{AD}[4]$, could directly interact with the high-affinity cholinergic transporter and impair steady-state and on-demand ACh release[5]. Treatment of this decrease undershoot of ACH in the synaptic cleft by using cholinesterase inhibitors, increases at the same time the cholinergic impact on bladder function, thereby worsen incontinence. Acetylcholine is one of the main neurotransmitters to control bladder function, both centrally as well as at peripheral levels. ACh has been shown to be released by the urothelium in response to chemical or mechanical stimulation in addition to the neuronal release as a (motor) nerve neurotransmitter. Hence, both, neuronal and non-neuronal ACh play a role in control of bladder function. Furthermore, sensory and motor function involve cholinergic signalling. Therefore, as well as for the dilemma in treating dementia and disfunction, cholinergic effects are an interesting target to study in a mouse model of Alzheimer's disease. Stimulation of the $\mathrm{M}_{1}$ muscarinic receptor in the cerebral cortex has shown to inhibit the micturition reflex pathway[6]. Down-regulation of this inhibitory mechanism plays an important role on $\mathrm{OAB}$ in Alzheimer type dementia[6]. $\mathrm{M}_{1}$ receptors are expressed in brain tissue. However, $\mathrm{M}_{2}$ and $\mathrm{M}_{3}$ receptors are expressed in the bladder. Xanomeline, $\mathrm{a}$ selective $\mathrm{M}_{1}$ and $\mathrm{M}_{4}$ muscarinic agonist did not produce parasympathomimetic effects in mice and rats after systemic administration. Xanomeline may be beneficial for the treatment of $\mathrm{AD}$, to prevent peripheral anticholinergic side effects [7]. The most common type of urinary incontinence in patients with $\mathrm{AD}$ is urge urinary incontinence secondary 
to bladder overactivity[8]. Overactive detrusor contraction (i.e. neurogenic detrusor overactivity)[9] or accelerated reflexes of the bladder muscle were considered to be the cause of the urinary incontinence in AD. It is important to distinguish between central and peripheral reasons behind urinary incontinence in AD patients. We aim for defining the effect of a cholinergic agonist as well as potassium as an indigenous cell membrane depolarizing ion, on isolated bladders of transgenic AD mice. This may shed light on the pathophysiology of cholinergic drug side effects and possible modalities of influence being altered by amyloid- $\beta$ in the bladder itself. The transgenic line we used(APPSL/PS1 ${ }^{\mathrm{M} 146 \mathrm{~L}}$ ) overexpress amyloid- $\beta$ in brain tissue[10]. In this study we aimed for defining the possible structural or physiological changes in bladders of AD mice. We examined two different chemical mediators of smooth muscle contraction. Accordingly we investigated the pathophysiology of evoked and spontaneous contractile activity in bladders from transgenic AD mice compared to bladders from WT mice. This study is for the first time assessing the pathophysiology of an AD model organism, using dissected bladders in an organ bath setting, with no CNS interference

\section{Methods:}

All procedures were approved by the Maastricht university animal ethical committee. First, animals were sacrificed by cervical dislocation. Bladders of 16 transgenic AD mice of the APPSL/PS1 ${ }^{\mathrm{M} 146 \mathrm{~L}}$ model and nine wild type mice were dissected. Twelve AD and seven WT bladders were catheterized transurethrally and placed in an organ bath containing carboxygenated Krebs buffer (NaCL 121,1mM; KCl 1,87mM; KH2P04 1,17mM; MgSO4 * 7H2O 1,15mM; NaHCO3 24,9mM; Glucose 11,0mM; CaCl2 * 2H20 1,2mM in a pH of 7.4 at $37^{\circ} \mathrm{C}$ ). After 30 minutes of rest the bladders were filled with $90 \mu \mathrm{l}(60 \%$ of total bladder capacity) of Krebs buffer. The organ bath was then washed 2 times for 5 minutes by refreshing the total amount of buffer. The bladders were then intravesically stimulated with $1 \mu \mathrm{M}$ carbachol (a nonselective muscarinic agonist). The organ bath was subsequently washed 2 times for 5 minutes. Subsequently the bladder was stimulated with $0.1 \mathrm{M} \mathrm{KCl}$ and the experiment was terminated. The intravesical pressure was measured by BIOPAC $\AA$ systems AcqKnowledge $\AA$ software. The frequency of asynchronized bladder smooth muscle contractions were measured from 2 minutes before until the start of carbachol infusion and between 3 to 5 minutes after the infusion. These microoscillatory function (figure 2) were filtered for noise. These frequencies were assessed by selection of contractile activity superseding $>0.1 \mathrm{cmH}_{2} \mathrm{O}$ after filtration for noise reduction. The area under the curve was measured by AcqKnowledge $₫$ analysis software built-in integral function within a specific time interval, from the beginning of the contraction until the peak amplitude following the stimulation with either carbachol or $\mathrm{KCl}$. The data were analyzed using paired samples t-test using Microsoft $®$ Excel®. Indirect immunohistochemical staining was performed on bladders of four AD mice and two WT mice. Strips were cut from the lateral wall, and fixed using 4\% paraformaldehyde for 2 hours at $4^{\circ} \mathrm{C}$. Tissue was fixed in three days of consecutive incubations at $4^{\circ} \mathrm{C}$ in 0.1 M phosphate buffer with $10 \%, 20 \%$ and $30 \%$ sucrose. $10 \mu \mathrm{m}$ sections perpendicular to 
the urothelial surface were made with cryostat. The sections were washed 3 times in TBST, TBS, TBST buffers and incubated for $24 \mathrm{~h}$ with the primary antibody (Human antiamyloid- $\beta$ and anti-APP diluted 1:4000). After washing in buffer, the sections were incubated by the secondary antibody (diluted 1:500 anti-human biotin; Jackson Immunoresearch Laboratories, West Grove, PA, USA) for 2 hours, and the ABC kit (avidinbiotin-peroxidase complex, diluted 1:400, Elite ABC-kit, Vestastatin, Burlingame, CA, USA) for 1.5 hour. The sections were then incubated with $\mathrm{DAB}\left(3,3^{\prime}\right.$-diaminobenzidine tetrahydrochloride)(50\% solution) with nickel chloride intensification for 10 minutes. Slides were washed, dehydrated and overlaid by coverslip using Pertex (Histolab Products ab, Goteborg, Sweden). An overview of the experimental protocol and readout parameters is depicted in figure 1.

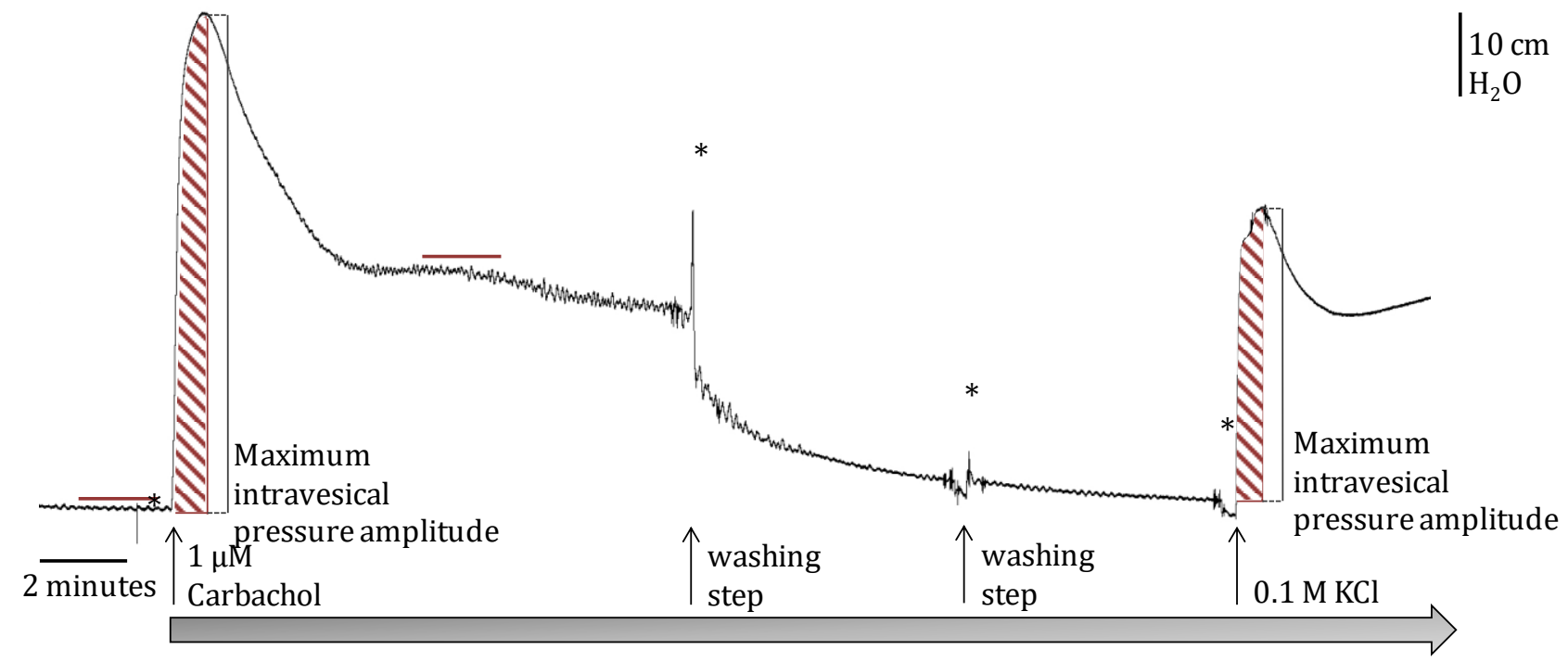

Figure 1. Experimental protocol and readout parameters. The pharmacological agents carbachol and $\mathrm{KCl}$ were administered at two subsequent occasions. The frequency of microcontractile activities were measured at two intervals before and after administration of the carbachol. The area under the curve was measured from the beginning of the contraction up to the peak of intravesical pressure for both carbachol and $\mathrm{KCl}$ (blue and red highlighted areas).

\section{Results:}

In order to investigate the muscarinic system, bladders were stimulated with carbachol and data sets were analysed based on the frequency of microcontractions. Representative traces of such smooth muscle activity are shown in figure 2 for bladders of WT and AD model mice. They were measured at equal time intervals relative to the start of stimulation with carbachol as detailed above. A decrease in frequency of asynchronized microcontractions was recorded during the baseline state in AD mice after stimulation with carbachol (p-value: 0.017)(figure 2-E). There was no difference between the two groups compared in standardized time intervals before muscarinic stimulation. As shown 
in figure 1 the intravesical pressure first reaches a sharp peak and then slowly declines, with increasing asynchronized activity.

A

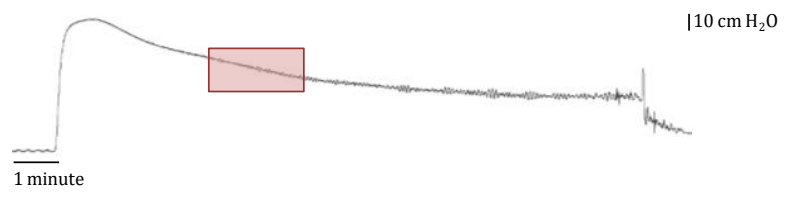

C

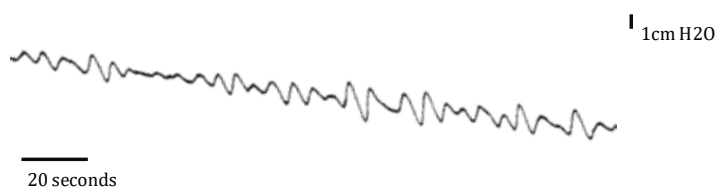

B

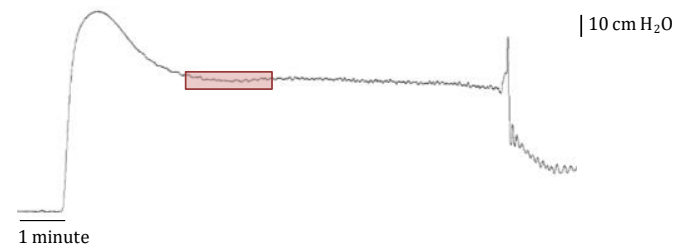

$\mathrm{D}$

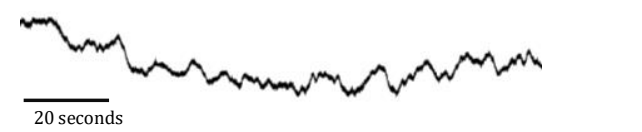

Frequency of Microcontractions

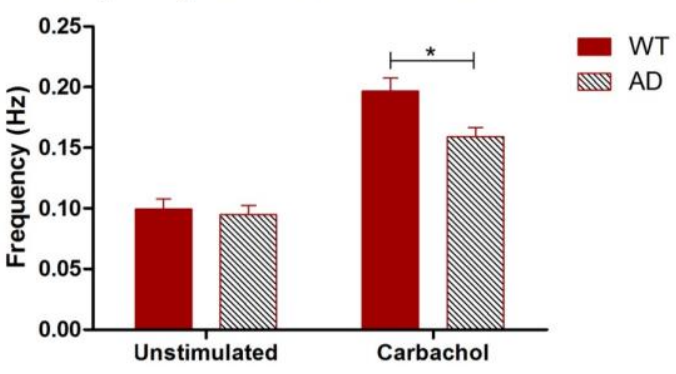

Figure 2. Representative traces of spontaneous microcontractions after the main peak of carbachol induced contraction.

A and B: Traces of wild type and Alzheimer's disease mice with their respective magnified views (C and D). E: The frequency of asynchronized microcontractions was significantly lower in bladders of AD mice after stimulation with Carbachol (p-value: 0.017). (WT: wild type, AD: Alzheimer's disease)

For investigating the depolarization capability of the detrusor smooth muscle, we compared the intravesical pressure amplitude and the AUC after stimulation with $\mathrm{KCl}$ between AD and WT mice. The intravesical pressure amplitude was not significantly different in two groups after either carbachol or $\mathrm{KCl}$ administration. The AUC was significantly lower in bladders from AD mice compared to WT mice after $\mathrm{KCl}$ administration (p-value: 0.048)(figure 3). There was no difference in the AUC between the two groups after carbachol stimulation. Both the frequency and the AUC as measures of bladder function, were lower in AD versus WT individuals.

After staining bladder tissue with anti-amyloid- $\beta$ and anti-APP antibodies, immunoreactivity was detected in the smooth muscle layer of the bladders of $A D$ mice(figure 4). WT mice revealed no immunoreactivity. 
A

Amplitude of Initial Contraction after Stimulation with Carbachol and $\mathrm{KCl}$

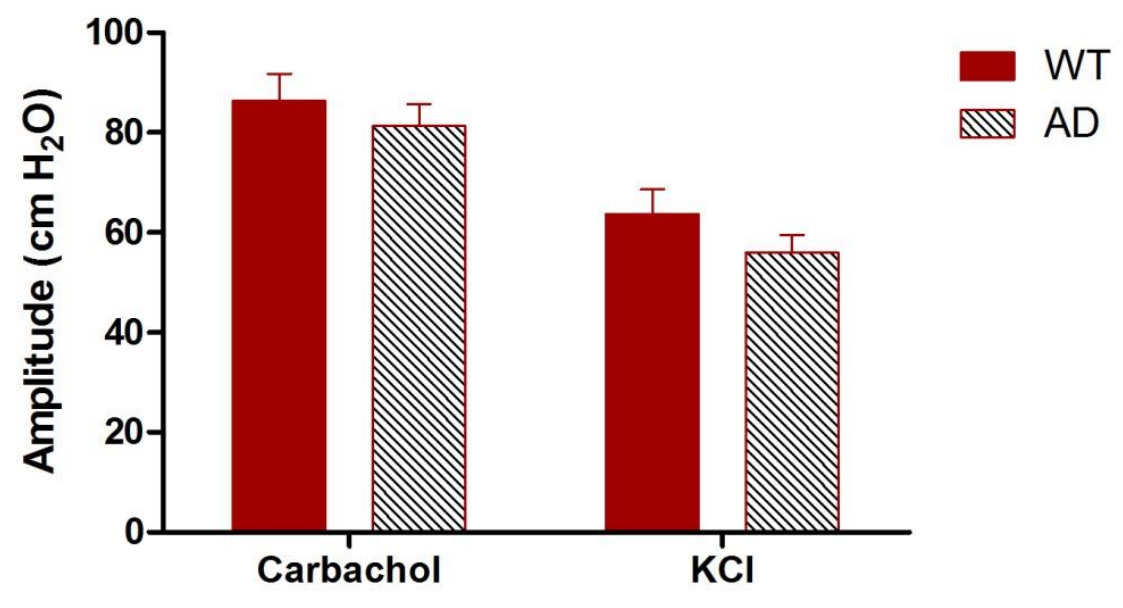

B
Area under the Curve (AUC) of Initial Contraction after Stimulation with Carbachol and $\mathrm{KCl}$

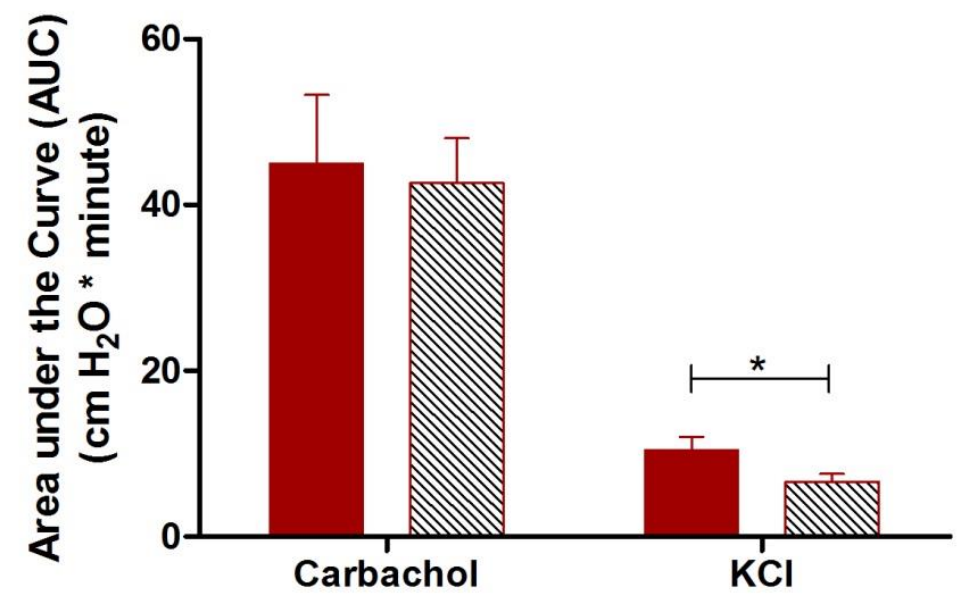

Figure 3. The comparison of detrusor contraction activity after either carbachol or KCl administration. Intravesical pressure amplitude (A) was not significantly different between AD or WT groups after either carbachol or $\mathrm{KCl}$ administration. The AUC (B) was significantly lower in bladders of $\mathrm{AD}$ mice compared to bladders of WT mice after $\mathrm{KCl}$ administration (p-value: 0.048). (WT: wild type, AD: Alzheimer's disease) 

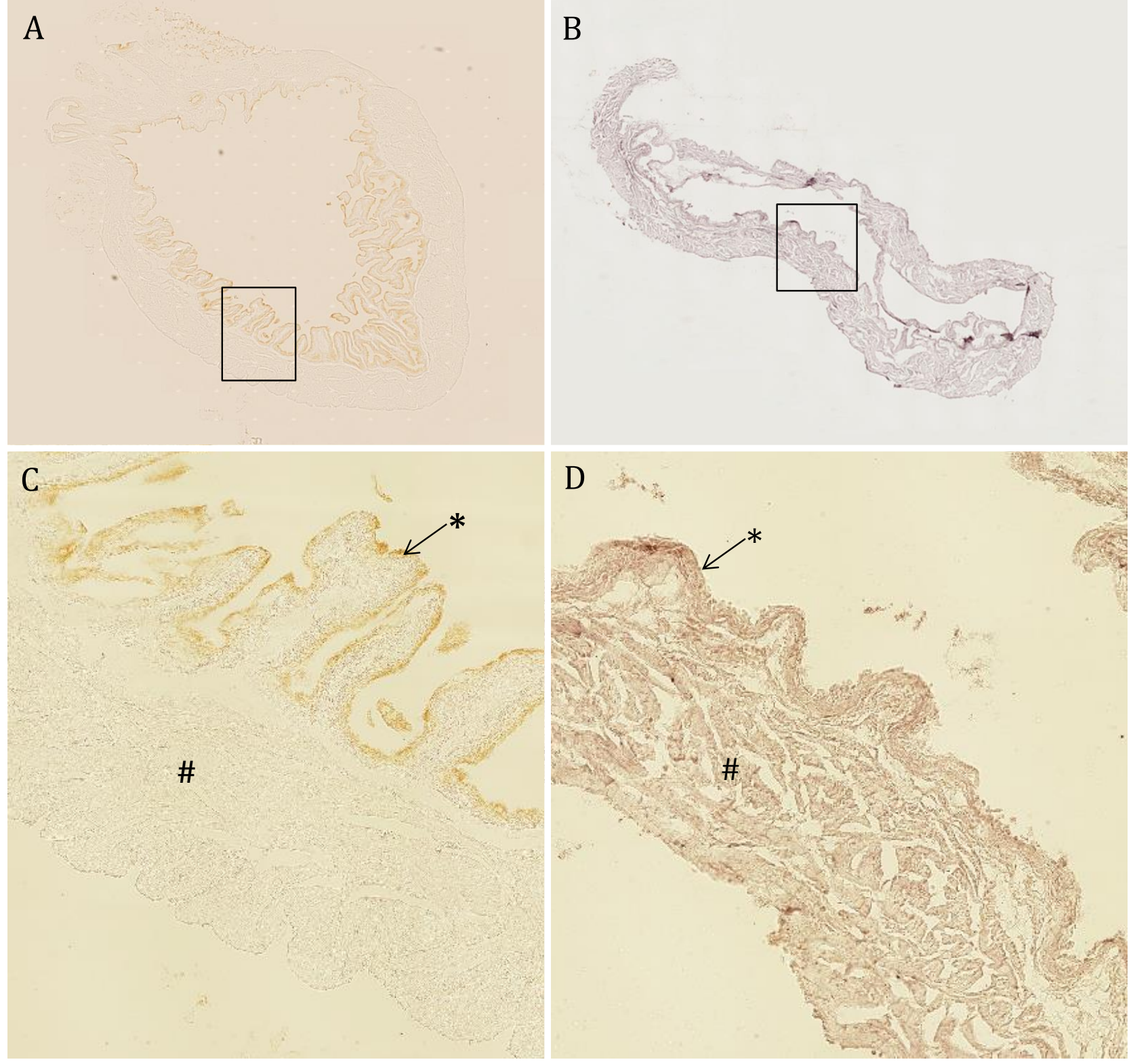

Figure 4. In contrast to control tissue (A, C), amyloid- $\beta$ or APP immunoreactivity is observed in the detrusor muscle of transgenic $A D$ mice $(B, D)$. The images are virtual slides taken the base magnification of $20 x$ objective $(A, B)$ with insets of the same magnification showing specific areas(C, D). There is some nonspecific cross reactivity at the WT mice epithelium. *: Transitional epithelium \#: Smooth muscle 


\section{Discussion}

Our data show functional deterioration in the bladders of $\mathrm{AD}$ mice corroborated by histological evidence of amyloid- $\beta$ deposition in detrusor muscle. The bladder dysfunction associated with $\mathrm{AD}$ may be due to either cognitive impertinence for voiding at the right occasion, pathology in brainstem micturition centers, or a defect in peripheral nerves or in bladder or sphincter musculature. Noncognitive etiologies may be secondary to amyloid deposition or other changes at a protein level and have pathophysiological consequences in the end organs. In this study, we showed for the first time differences in the function of isolated bladders in AD mice compared to WT mice in an organ bath setting without any CNS interference.

Structural changes in bladders of AD mice have been shown before, for example by the alterations of the location of intramural ganglia within AD mouse bladders[11]. There is also evidence for the existence of amyloid- $\beta$ in non-nervous tissues in post-mortem humans [4]. Other peripheral tissues including the urinary bladder failed to demonstrate any APP-positive immunoreactivity in humans[4]. In our study bladder smooth muscle tissue showed immunoreactivity to amyloid- $\beta$ and APP. There's no evidence in favour of the capability of axonal migration of amyloid- $\beta$ for example from the brain, in the literature. So amyloid- $\beta$ is most probably indigenously expressed in cells with AD mutations. All of the embryonic cell lines are infected in transgenic animal models, confirmed in our study by the presence of amyloid- $\beta$ in the bladder. These findings were not ratified yet in human studies which might be explained by the sporadic nature of Alzheimer cases in the human subjects tested. This is in contrast to some familial clusters of AD in human.

Various possible reasons exist behind the alteration of the bladder function in AD mice. Based on these findings we can not confirm which would be the exact etiology, but some previous research have pointed to cellular and molecular alterations in AD cells. Both Calcium and Potassium ions are involved in smooth muscle contraction cycle. Potassium channel malfunction can be found in various tissues of AD patients[12] [13] [14]. Some specific forms of this channel which are present in smooth muscles[15, 16], are upregulated[17-19] in AD individuals, resulting in apoptosis[20]. Upregulation of ryanodine calcium receptors in transgenic Alzheimer mice[21], are another example of such subcellular alterations. The microcontractile smooth muscle activities may represent asynchronized smooth muscle contractions secondary to tissue denervation after dissection of the bladder, leaving the smooth muscle bundles in disharmony. The frequency of these contractions were found to be lower in transgenic AD mice after carbachol stimulation compared to the bladders of WT mice. In contrast to the amplitude, the AUC was significantly decreased in the bladders of transgenic AD mice after $\mathrm{KCl}$ administration. The AUC adds the time interval to intravesical pressure measurement, which is related to total energy expenditure by the tissue, and defines the capability of the tissue to sustain a particular contractile force. That may be a reason why pure intravesical pressure did not change in the two groups. It is noteworthy to mention that 
this indicates a different phenomenon than the detrusor overactivity of $\mathrm{OAB}$, since overactivity does not imply increased force during agonist evoked contraction, but increased frequency of contractions.

\subsection{Conclusions}

The reason for bladder dysfunction in neurodegenerative disorders was usually attributed to changes in central control of bladder physiology. This may be secondary to cognitive decline or damage to micturition control centers. We observed differences in bladder function in AD mice compared to WT mice in an organ bath setting without any CNS interference. Histological evidence of AD markers points to the fact that there might be some direct influence of amyloid- $\beta$ on bladder physiology. In clinical grounds, these alterations may exist in hereditary forms of subjects with AD.

\section{References}

1. Porter, R.S., The Merck Manual of Diagnosis and Therapy. 2011: Wiley.

2. DelSer, T., D.G. Munoz, and V. Hachinski, Temporal pattern of cognitive decline and incontinence is different in Alzheimer's disease and diffuse Lewy body disease. Neurology, 1996. 46(3): p. 682-686.

3. Ropper, A., M. Samuels, and J. Klein, Adams and Victor's Principles of Neurology 10th Edition. 2014: McGraw-Hill Education.

4. Arai, H., et al., Expression patterns of beta-amyloid precursor protein (beta-APP) in neural and nonneural human tissues from Alzheimer's disease and control subjects. Ann Neurol, 1991. 30(5): p. 686-93.

5. Bales, K.R., et al., Cholinergic dysfunction in a mouse model of Alzheimer disease is reversed by an anti-A beta antibody. Journal of Clinical Investigation, 2006. 116(3): p. 825-832.

6. Yokoyama, O., et al., Overactive bladder - Experimental aspects. Scandinavian Journal of Urology and Nephrology, 2002. 36: p. 59-64.

7. Bymaster, F.P., et al., Xanomeline: A selective muscarinic agonist for the treatment of Alzheimer's disease. Drug Development Research, 1997. 40(2): p. 158-170.

8. Lee, S.H., et al., Urinary incontinence in patients with Alzheimer's disease: Relationship between symptom status and urodynamic diagnoses. International Journal of Urology, 2014. 21(7): p. 683-687.

9. Sugiyama, T., et al., Urinary incontinence in senile dementia of the Alzheimer type (SDAT). Int J Urol, 1994. 1(4): p. 337-40.

10. Duff, K., et al., Increased amyloid-beta42(43) in brains of mice expressing mutant presenilin 1. Nature, 1996. 383(6602): p. 710-3.

11. Biallosterski, B.T., et al., Changes in bladder innervation in a mouse model of Alzheimer's disease. J Chem Neuroanat, 2010. 39(3): p. 204-10.

12. M. Boakye, R.E., V. Lubierman, B. Wolozin, D. L. Alkon. , TEA and Ca2+ sensitivity of Potassium Channels in Olfactory Neuroblasts from Alzheimer's and Normal Donors. Soc. Neurosci. . p. 1573. 
13. de Silva, H.A., et al., Abnormal function of potassium channels in platelets of patients with Alzheimer's disease. Lancet, 1998. 352(9140): p. 1590-3.

14. Etcheberrigaray, R., et al., Potassium channel dysfunction in fibroblasts identifies patients with Alzheimer disease. Proc Natl Acad Sci U S A, 1993. 90(17): p. 8209-13.

15. Miguel-Velado, E., et al., Contribution of Kv channels to phenotypic remodeling of human uterine artery smooth muscle cells. Circ Res, 2005. 97(12): p. 1280-7.

16. Miguel-Velado, E., et al., Cell cycle-dependent expression of Kv3.4 channels modulates proliferation of human uterine artery smooth muscle cells. Cardiovasc Res, 2010. 86(3): p. 383-91.

17. Pannaccione, A., et al., Up-regulation and increased activity of KV3.4 channels and their accessory subunit MinK-related peptide 2 induced by amyloid peptide are involved in apoptotic neuronal death. Mol Pharmacol, 2007. 72(3): p. 665-73.

18. Choi, E. and G.W. Abbott, The MiRP2-Kv3.4 potassium channel: muscling in on Alzheimer's disease. Mol Pharmacol, 2007. 72(3): p. 499-501.

19. Angulo, E., et al., Up-regulation of the Kv3.4 potassium channel subunit in early stages of Alzheimer's disease. J Neurochem, 2004. 91(3): p. 547-57.

20. Yu, S.P., Regulation and critical role of potassium homeostasis in apoptosis. Prog Neurobiol, 2003. 70(4): p. 363-86.

21. Stutzmann, G.E., et al., Enhanced ryanodine receptor recruitment contributes to Ca2+ disruptions in young, adult, and aged Alzheimer's disease mice. J Neurosci, 2006. 26(19): p. 5180-9. 


\section{Chapter 8}

General discussion 
In a multidisciplinary project we combined neuroanatomical, neurochemical and neurophysiological studies, to gain a better understanding of the connections to and from the periaqueductal grey matter, related to bladder afferent input. Here we first reexamined the specific PAG columns which receive sensory bladder signals, then aimed for defining the particular cell types within the involved column and finally tried to define the electrophysiological characteristics of the vlPAG.

In chapter 2 of this thesis we made an in depth review of the literature to unravel the state of the PAG research in functional urology. Our goal was to find out whether there is a particular pattern that would explain the internal circuits present in the brainstem, which influence the autonomic control of the micturition and the relay of signals to cortical centers. We also described various pathological conditions related to the PAG which would affect the micturition cycle. Various imaging modalities which may detect PAG lesions and their possible clinical application were also discussed. The shortcomings of current imaging techniques is their relatively low spatial resolution specially regarding differentiation of various PAG columns. On the other hand the basic tractology of the PAG connections is not well understood. This deficiency in the anatomical perspective would be important regarding the extensive connections that the PAG has with the brainstem and the cortical areas. We also differentiated the role of two critical PAG columns namely ventrolateral (vlPAG) and the dorsolateral (dIPAG) on the physiology of micturition. This is in line with previous studies defining opposite autonomic functions of these columns. The urogenital system, rich in autonomic innervation is also influenceed by these two columns as contraction or relaxation of detrusor, relaxation or contraction of internal urethral sphincter and erection and ejaculation in men are all controlled by parasympathetic and sympathetic signals originating from the ventrolateral and the dorsolateral columns of the PAG respectively.

In chapter 3 we tried to develop a surgical method which would make it possible for the experimentalist to implement neuromodulation in freely moving rats. Our main goal was to ensure that our stimulation paradigm would not make adverse behavioral outcomes. Since the PAG is involved in controlling different aspects of homeostasis like nociception, behaviour and autonomic control, any influence of each of them must be differentiated by behavioural tests. To tackle this we took advantage of the rodents' resilient skin jacket with a relatively large subcutaneous space, to guide wires, transferring them from the organ of interest(in this case the urinary bladder) to a safe place(over the skull) which can then be connected to a surface plug. Our results showed that rats can fairly tolerate this subcutaneous implantation technique with no pain or anxiety after 2 weeks of recovery. This technique makes it easy for them to move naturally while being stimulated and can be used for other similar experiments dealing with stimulation protocols or introducing particular chemicals, while the main access point for the experimentalist is over the skull of the animal. This also keeps the access point out of the reach of the animal which would have otherwise been manipulated and damaged. 
Chapter 4 uses the technique mentioned above to electrically stimulate the bladder. The rat would simultaneously undergo behavioural tests to find out any influence on the behaviour. In this way we could differentiate the various parameters that would lead to PAG activation. Our goal was to find out if a particular PAG column would be activated after bladder stimulation, simulating sensory signals. By performing behavioural tests we could exclude pain and anxiety as other influencing factors leading to PAG activation. We performed c-Fos staining of the PAG and found that the ventrolateral column is significantly activated in stimulated groups versus the sham group. This may imply that vlPAG is the PAG station for ascending sensory signals reaching from the bladder. Due to immense intercolumnar connections, these inputs may secondarily be relayed to other PAG columns. Other possible destinations of sensory bladder signals are projections from vlPAG to the pontine micturition center. In higher order animals with more sophisticated decision making capabilities, these signals may then be transferred to specific cortical regions for further analysis.

As a next step in chapter 5 we aimed for defining the particular cell types that may be involved in receiving bladder sensory signals. We focused on the vlPAG as it was the column defined in the previous step to be responsible for sensory bladder processing. We examined five different neurotransmitter markers including dopamine, serotonin, nNOS, Glutamate and GABA by co-labelling them with c-Fos to find out which neurotransmitter systems are involved. The involvement would mean that the c-Fos activated neurons are of a particular type, within vlPAG that can be defined as the station of incoming bladder signals. We found glutamatergic vlPAG cells to be significantly activated in the stimulated group. As glutamate is a stimulatory neurotransmitter, these neurons can be considered to stimulate another center in response to sensing a full bladder. One hypothesis is that these glutamatergic cells are the ones projecting to the pontine micturition center, activating them, and ultimately, when strong enough, would lead to bladder contraction. The firing pattern of these neurons would probably change after receiving suprathreshold sensory bladder signals., which would be the main topic of the next chapter.

In chapter 6 we aimed for electrophysiological characterization of the PAG neurons and evaluation of their response to electrical bladder stimulation. To accomplish this we used the same electrical stimulation parameters that we had defined in chapter 4 . We subsequently recorded neuronal firing before, during and after about 1 minute stimulation period. We then evaluated whether any particular response type could be identified in the neuronal firing pattern. The problem with this technique is that, despite the neurons recorded are located in the vlPAG, they are selected randomly and some of them may be responsible for non-micturition functions. The results show that the overall firing frequency of the recorded neurons significantly decreaseed during the stimulation period. This at least means an overall response of vlPAG neurons to ascending bladder signals. Considering that the stimulation parameters are set for a range which would not activate pain and anxiety reactions, we may conclude that this response is more likely to influence micturition related neurons. We may not be able to fully correlate this to our 
previous c-Fos experiment since we were only evaluating neuronal activation there. cFos is an activation marker, but if perhaps an inhibition marker would have existed and been implemented, another group of neurons corresponding to our electrophysiological inhibition findings would have possibly been revealed. With these data we can not comment on the type of neuronal population that has been recorded. Moreover, there is ample evidence that other activities such as neurotransmitter release, do not correlate with firing pattern. To achieve the best results, the found neuron could have been juxtaglomerularly labelled for a particular neurotransmitter to find out the exact cell type. On the other hand electrophysiological distinction of cell types by characteristics of their extracellular action potential is difficult. The action potentials we recorded may have been from somites, dendrites, synaptic points or axons. Of these, the most likely place of the recordings was the axons as they have a far larger surface area than the other regions. Though the shape of the action potentials vary in different axons, these variation would most likely not reflect the difference of their neurotransmitter content. The shape of the action potentials is mostly determined by the Sodium and Potassium currents in the axon. As seen by the regression analysis, we could not differentiate the neurons solely by the characteristics of the shape of their action potential, either peak/trough ratio or peaktrough latency. On the other hand, such differences especially between glutamatergic and GABAergic neurons which are stimulatory and inhibitory would have probably been more distinguishable if recorded at the synaptic interface. Even if it were possible to locate a synapse, and record the extracellular action potential, it is not possible to differentiate between a glutamatergic and GABAergic neuron, as any other excitatory or inhibitory synaptic interface may have a similar tracing. Another possible way to electrophysiologically distinguish different neurons is measurement of their axonal action potential propagation speed, which is technically different.

We also explained a more efficient way to confirm the place of the recorded neurons. To our best knowledge this is the first paper demonstrating iontophoretically injected pontamine to be located by fluorescent observation. To achieve this we used Hoechst as background fluorescence. This aided to delineate the borders of the PAG. Even trace amounts of the pontamine stain are visible in red fluorescent spectrum. This tactic helps to reduce the amount of injected pontamine and though make a more precise localization. Merging red and blue filter spectra will demonstrate the exact point of injection.

The leading causes of morbidity in the elderly population is neurodegenerative diseases including Alzheimer's disease (AD). Before trying to modulate bladder physiology by therapeutic experiments involving the PAG in $\mathrm{AD}$, we wanted to confirm that PAG pathologies correspond to bladder malfunction in this disorder. In chapter 7 , to make a relevant experimental setup, we omitted the influence of the central nervous system, by solely focussing on the peripheral organ, in an organ bath setting. This paves the way for pure evaluation of bladder physiology. Our experiment showed both structural and physiological alterations in AD mice bladders. This points to the fact that local $\beta$-amyloid related pathological changes within the detrusor muscle or the associated pelvic innervations may independently modulate bladder function. Other more widely accepted 
reasons were cognitive impertinence to voiding behavior or pathology in brainstem micturition centers. It's not the first time $\beta$-amyloid related structural changes are reported in non-nervous tissue[1]. This in particular is of importance in familial clusters of human $\mathrm{AD}$ subjects with a genetic background. How accumulation of $\beta$-amyloid may affect smooth muscle physiology had been described by its effect on voltage gated calcium or potassium channels[2,3]. In fact such channel defects are present in various AD cell lines[4-6]. On the other hand, most AD cases are sporadic. Consequently the $\beta$-amyloid genetic mutations would most likely be limited to the brain in sporadic cases.

\section{The role of the vlPAG in micturition}

As a coordinating structure between evolutionary higher and lower levels of the central nervous system, the vlPAG has a broad spectrum of functions. It plays the role of a switchboard connecting evolutionary primitive and advanced brain centers with each other. Its internal intercolumnar connections complete a full input-output circuit. Hence pathologies affecting this structure would affect micturition[7-10]. This highlights the potential diagnostic and therapeutic role of the PAG in functional urological disease. The role of the vlPAG is important in the aspect of its connections with sacral segments as well as the pontine micturition center. Though vlPAG itself may not have extensive connections with the cortex, its connections with other PAG columns makes it a node which can subsequently relay all this information to almost any other part of the brain. Moreover its proximity to the central aqueduct makes it a structure, possibly sensitive to chemical alterations of the cerebrospinal fluid which can sometimes have great physiological impacts. For example, changes in the $\mathrm{pH}$ of the cerebrospinal fluid(secondary to changes in blood $\mathrm{pH}$ ) has instantaneous effects on respiratory centers within the brainstem which adjust the rate and depth of breathing to compensate for any amount of $\mathrm{CO}_{2}$ retention. On the other hand the second most important organ for controlling blood pH after lungs, are the kidneys. But no research has so far been performed to find out if such alterations in $\mathrm{pH}$ would perhaps, by means of PAG influence, alter renal glomerular blood flow or filtration controls. This is somewhat away from our topic, but still emphasizes that research on the PAG to study various physiological functions merits more consideration. It's important to differentiate between different PAG columns and to find out which column is responsible for a particular function. Unfortunately, so far there has been no sophisticated anatomical, histochemical method to be able to delineate the borders of various PAG columns in a standard fashion. The existence of various columns has been suggested by physiological studies, and was subsequently implemented in brain atlases. Our initial aim was also to confirm the location of a particular column which may handle ascending sensory signals from the bladder. We found the ventrolateral column to be responsible for this. Presumably this column receives the information in a lateralized fashion, but this is very difficult to confirm. Though since the bladder is a small central viscus, the lateralization may not be that important. vIPAG in contrast to the dorsolateral column is responsible for the 
parasympathetic discharge toward the organs. For example vlPAG activation will induce bradycardia, hypotension and hypoventilation. So we can extrapolate that vIPAG activation can contract the bladder, much the same way as the parasympathetic activation does. One high resolution rabbit fMRI study actually confirmed the ventrolateral column of the PAG to be activated after bladder stimulation[11]. The special role of the vlPAG respecting the control of the bladder function is derived from 3 types of studies in general:

- Anatomical studies: These studies use tracing techniques to find out which PAG column has connections with sacral parasympathetic segments responsible for bladder control.

- Functional(neurophysiological) studies: These studies either stimulate a particular PAG column and monitor intravesical pressure or stimulate the bladder and evaluate the PAG reaction to these stimulations in various ways(similar to our study).

- Functional imaging: These are usually done in human subjects and often implement fMRI for investigation, though PET or SPECT are other modalities. This type of study has important challenges and promises which merit consideration.

One of the major shortcomings of functional imaging is their lack of enough spatial resolution particularly to differentiate between various PAG columns. One important advantage of this type of study over other variants described above is their ability to investigate the PAG and other brain areas in specific pathological conditions affecting the bladder function. Using fMRI it has been found that vIPAG handles the bladder filling sensation differently from noxious bladder stimuli[12, 13]. Experimental studies implementing pelvic muscle contraction or rectal distension in normal individuals showed PAG activation. This implies that the PAG is particularly involved in pelvic organ functions. PAG function is also in coordination with the function of some particular cortical areas namely the insula, prefrontal cortex and anterior cingulate gyrus. The PAG has specially been found by fMRI studies to be activated during initial moments of micturition and even moments before start of the micturition. This emphasizes its role as a kind of on-off switch to turn on a green light for the urinary let-down[14].

The ventrolateral column is the PAG segment mostly connected with the bladder[15-20]. vlPAG glutamatergic cell groups may have descending motor connections with stimulatory role on bladder[21]. On the other hand dIPAG GABAergic cells have a reverse function inhibiting bladder contraction[22]. These are in addition to other functions attributed to these cell types, including freezing behavior and nociception[23]. The direct connections of the vIPAG with sacral cord[24] and PMC makes it an intermediate node completing an input-output circuit. This makes the vlPAG a center for control of reflexive micturition in rodents and probably in the immature human infant. In our experiment vlPAG glutamatergic cells were activated after electrical bladder stimulation. These glutamatergic cells receive peripheral sensory signals and will probably relay them to the PMC. They may initiate a micturition reflex after receiving suprathreshold sensory signals 
from a full bladder. Relay to cortical regions by glutamatergic cells may also occur for decision making especially in human. Pathological evidence for involvement of these cell types is present in multiple system atrophy (MSA). This disease has micturition problems accompanied by decreased number of glutamatergic cells in PAG columns[10]. We did not find any evidence for activation of other cell types within vlPAG. Intercolumnar connections within the PAG may provide influence from other cell types from adjacent segments[22]. Dopaminergic, serotoninergic and nitric oxide(NO) bearing cells were also revealed in vlPAG. Dopaminergic and serotoninergic cells are particularly abundant in dorsal raphe region but are scant throughout PAG columns. NO on the other hand is abundant in vlPAG but NO synthesizer cells were not affected by electrical bladder stimulation. On the other hand NO bearing cells are very active in dorsal PAG regarding autonomic, behavioral and nociceptive control.

PAG may, due to its extensive connections with cortical, diencephalic, brainstem and spinal cord regions[17, 24-31], serve as a hot spot for electrophysiologic investigations. Two main columns in PAG were distinguished (ventrolateral and dorsolateral) to be involved in bladder control. vlPAG is more involved in motor control by activation of the bladder contraction and dIPAG has an inhibitory role[22]. vlPAG is the major column with anatomical connections to the bladder afferents and also to the central micturition centers $[24,32,33]$. Previous studies had shown different neuronal firing rates related to the micturition[34]. Studies also show altered firing patterns in the PAG in stimulated sleeping states[35], so there's a possibility of electrophysiologic alterations in nocturnal enuresis[35]. We investigated the neuronal firing pattern of this column after electrical bladder stimulation to mimic sensory signal processing.

\section{Possible clinical implications}

The PAG may be affected in various CNS pathologies like myelin disorders(as multiple sclerosis), complex biochemical or metabolic disorders(Wernicke's encephalopathy[36] or Parkinson's disease(PD)[7]) and structural disorders(like stroke or tumors). In all these disorders, voiding dysfunction is present at some extent. This highlights the clinical importance of the PAG and its vacancy in most diagnostic and therapeutic algorithms dealing with micturition problems. fMRI studies have shown that sacral neuromodulation, as a common treatment modality in specifically selected patients, is accompanied by changes in the activity of the PAG. This means that ascending sensory pathways exist, reaching from the pelvic organs to the $P A G$, which after neuromodulation may reconfigure the most prominent central location of the micturition control[37].

Diagnostic tools are currently not efficient enough to be able to fully distinguish PAG pathologies. Except for research purposes, functional imaging has almost never been used for the sole purpose of diagnosing the PAG pathology. Meanwhile more sophisticated imaging techniques like magnetic resonance spectroscopy(MRS) have the potential to detect the content of specific metabolites within the PAG. As described in this 
thesis, glutamate was a crucial metabolite within the PAG, which is involved in micturition function. Alteration of glutamate metabolism or expression pattern in the PAG may be evaluated by MRS in selected patients. Meanwhile MRS may be used to investigate other metabolites within the PAG and in relation to particular patient groups. This is in contrast to all imaging research done so far on the PAG, that concentrated solely on anatomical lesions or pathway investigation.

This provides a pathway to the PAG as a potential target for therapeutic interventions. The involvement of glutamatergic vIPAG cells in receiving sensory bladder signals may be exploited by targeting specific glutamate receptors by specific agonist or antagonist medications. Or by modulation of the metabolic pathway leading to glutamate synthesis. Recognition of specific metabolic isoforms active in the PAG may aid in more specific drug targeting. Potentially such drugs may alter the threshold of sensation in patients with sensory pathway dysfunction, or help to trigger or facilitate the voiding in patients with voiding dysfunctions.

In contrast, the PAG can also be a target for surgical intervention, particularly deep brain stimulation(DBS). This is particularly useful for functional bladder disorders which have a central etiology. The advantage of DBS is its capability of temporal adjustment corresponding to acute patient symptoms. However, the brainstem and particularly the PAG are risky areas for surgical and electrical manipulation and especially implantation of DBS electrodes. The PAG, encircling the central aqueduct, has a potential for inflammatory reactions around the electrodes, obstruction of the aqueduct and ultimately producing a non-communicating hydrocephalus and intracranial hypertension. So any attempt for using conventional DBS in this regions merits considerable risk-benefit assessment of their grave side effects. On the bright side, promising non-invasive DBS techniques like magnetothermal DBS are under extensive research. Their possible implementation in the future particularly in critical locations as the PAG would open new therapeutic opportunities on the horizon.

\section{Future perspectives}

The special role of PAG in micturition disorders can be exploited in either diagnostic or therapeutic aspects. fMRI of PAG in conjunction with urodynamic studies can yield invaluable insight of the state of central urological control. Targeting specific PAG columns and cell types, as described in this thesis, by deep brain stimulation or pharmacotherapy may alleviate the sufferings of some patients. Some questions still remain to be answered. For example, which vlPAG cell types are in connection with other PAG columns, pons and cortical regions, and how would each of these tracts modulate the activity of the detrusor, sphincter and overall continence (i.e. contraction or inhibition of the lower urinary tract structures). 


\section{Conclusion}

The main aim of the study was to find out the specific role of the PAG in sensory bladder signal processing. This study would give us a broader view of the role of the PAG in micturition problems and drive us to novel approaches for their management. In brief, the exact anatomical location of incoming afferent signals from the bladder to the PAG was assessed, cell types of the neurons that may handle the bladder afferent signals were characterized, and electrophysiologic characteristics of PAG neurons receiving sensory bladder stimulation, were determined. This has shed some light on how the PAG may handle bladder sensation. However this study did not give an idea of where these signals are relayed thereafter or if these signals are amplified or inhibited. This is important for both basic and clinical perspectives since a lot of central etiologies of voiding dysfunction may involve the PAG. Most of the current therapeutic options for voiding dysfunction are focussed on the peripheral nervous system of the target organs, while many have neurological or even psychological etiologies residing in the central nervous system.

\section{References}

1. Arai, H., et al., Expression patterns of beta-amyloid precursor protein (beta-APP) in neural and nonneural human tissues from Alzheimer's disease and control subjects. Ann Neurol, 1991. 30(5): p. 686-93.

2. Angulo, E., et al., Up-regulation of the Kv3.4 potassium channel subunit in early stages of Alzheimer's disease. J Neurochem, 2004. 91(3): p. 547-57.

3. Demuro, A., I. Parker, and G.E. Stutzmann, Calcium signaling and amyloid toxicity in Alzheimer disease. J Biol Chem, 2010. 285(17): p. 12463-8.

4. M. Boakye, R.E., V. Lubierman, B. Wolozin, D. L. Alkon., TEA and Ca2+ sensitivity of Potassium Channels in Olfactory Neuroblasts from Alzheimer's and Normal Donors. Soc. Neurosci. . p. 1573.

5. de Silva, H.A., et al., Abnormal function of potassium channels in platelets of patients with Alzheimer's disease. Lancet, 1998. 352(9140): p. 1590-3.

6. Etcheberrigaray, R., et al., Potassium channel dysfunction in fibroblasts identifies patients with Alzheimer disease. Proc Natl Acad Sci U S A, 1993. 90(17): p. 8209-13.

7. Herzog, J., et al., Improved sensory gating of urinary bladder afferents in Parkinson's disease following subthalamic stimulation. Brain, 2008. 131(Pt 1): p. 132-45.

8. Kitta, T., et al., Brain activation during detrusor overactivity in patients with Parkinson's disease: A positron emission tomography study. Journal of Urology, 2006. 175(3): p. 994998.

9. Betts, C.D., R. Kapoor, and C.J. Fowler, Pontine Pathology and Voiding Dysfunction. British Journal of Urology, 1992. 70(1): p. 100-102.

10. Benarroch, E.E., et al., Differential involvement of the periaqueductal gray in multiple system atrophy. Auton Neurosci, 2010. 158(1-2): p. 111-7. 
11. Xiang, B., et al., Functional Brainstem Changes in Response to Bladder Function Alteration Elicited by Surgical Reduction in Bladder Capacity: A Functional Magnetic Resonance Imaging Study. Journal of Urology, 2010. 184(5): p. 2186-2191.

12. Matsuura, S., et al., Human brain region response to distention or cold stimulation of the bladder: A positron emission tomography study. Journal of Urology, 2002. 168(5): p. 20352039.

13. Mehnert, U., et al., The Supraspinal Neural Correlate of Bladder Cold Sensation-An fMRI Study. Human Brain Mapping, 2011. 32(6): p. 835-845.

14. Kuhtz-Buschbeck, J.R., et al., Control of bladder sensations: An fMRI study of brain activity and effective connectivity. Neuroimage, 2009. 47(1): p. 18-27.

15. Mitsui, T., et al., Chemical bladder irritation provokes c-fos expression in the midbrain periaqueductal gray matter of the rat. Brain Res, 2003. 967(1-2): p. 81-8.

16. Duong, M., J.W. Downie, and H.J. Du, Transmission of afferent information from urinary bladder, urethra and perineum to periaqueductal gray of cat. Brain Research, 1999. 819(12): p. 108-119.

17. Taniguchi, N., et al., $A$ study of micturition inducing sites in the periaqueductal gray of the mesencephalon. J Urol, 2002. 168(4 Pt 1): p. 1626-31.

18. Stone, E., J.H. Coote, and T.A. Lovick, Effect of electrical vs. chemical deep brain stimulation at midbrain sites on micturition in anaesthetized rats. Acta Physiologica, 2015. 214(1): p. 135-145.

19. Matsuura, S., G.V. Allen, and J.W. Downie, Volume-evoked micturition reflex is mediated by the ventrolateral periaqueductal gray in anesthetized rats. Am J Physiol, 1998. 275(6 Pt 2): p. R2049-55.

20. Matsuura, S., J.W. Downie, and G.V. Allen, Micturition evoked by glutamate microinjection in the ventrolateral periaqueductal gray is mediated through Barrington's nucleus in the rat. Neuroscience, 2000. 101(4): p. 1053-1061.

21. Verstegen, H., et al., Brain Networks Controlling Bladder Filling and Voiding. Journal of Urology, 2017. 197(4): p. E1150-E1150.

22. Numata, A., et al., Micturition-suppressing region in the periaqueductal gray of the mesencephalon of the cat. American Journal of Physiology-Regulatory Integrative and Comparative Physiology, 2008. 294(6): p. R1996-R2000.

23. Samineni, V.K., et al., Divergent Modulation of Nociception by Glutamatergic and GABAergic Neuronal Subpopulations in the Periaqueductal Gray. eNeuro, 2017. 4(2).

24. Ding, Y.Q., et al., Direct projections from the lumbosacral spinal cord to Barrington's nucleus in the rat: a special reference to micturition reflex. J Comp Neurol, 1997. 389(1): p. 149-60.

25. Griffiths, D.J., Use of functional imaging to monitor central control of voiding in humans. Handb Exp Pharmacol, 2011(202): p. 81-97.

26. Griffiths, D., Chapter 7 - Functional imaging of structures involved in neural control of the lower urinary tract, in Handbook of Clinical Neurology, B.V. David and B. François, Editors. 2015, Elsevier. p. 121-133.

27. Mtui, E., G. Gruener, and P. Dockery, reticular formation, in Fitzgerald's Clinical Neuroanatomy and Neuroscience

2015, Elsevier.

28. Griffiths, D., Neural control of micturition in humans: a working model. Nat Rev Urol, 2015. 12(12): p. 695-705. 
29. Kuipers, R., L.J. Mouton, and G. Holstege, Afferent projections to the pontine micturition center in the cat. Journal of Comparative Neurology, 2006. 494(1): p. 36-53.

30. Klop, E.M., et al., Neurons in the lateral sacral cord of the cat project to periaqueductal grey, but not to thalamus. Eur J Neurosci, 2005. 21(8): p. 2159-66.

31. Kuipers, R. and E.M. Klop, Neurons in the guinea pig (Cavia porcellus) lateral lumbosacral spinal cord project to the central part of the lateral periaqueductal gray matter. Brain Res, 2006. 1101(1): p. 43-50.

32. Blok, B.F., H. De Weerd, and G. Holstege, Ultrastructural evidence for a paucity of projections from the lumbosacral cord to the pontine micturition center or M-region in the cat: a new concept for the organization of the micturition reflex with the periaqueductal gray as central relay. J Comp Neurol, 1995. 359(2): p. 300-9.

33. Mouton, L.J. and G. Holstege, Segmental and laminar organization of the spinal neurons projecting to the periaqueductal gray (PAG) in the cat suggests the existence of at least five separate clusters of spino-PAG neurons. J Comp Neurol, 2000. 428(3): p. 389-410.

34. MATSUMOTO Kengo, S.T., YAMAMOTO Gen Characterization of micturition-related single neuron activities and their distribution in periaqueductal gray matter (PAG) in anesthetized rats. J Pharmacol Sci 2006. 100: p. 251.

35. Crook, J. and T. Lovick, Urodynamic function during sleep-like brain states in urethane anesthetized rats. Neuroscience, 2016. 313: p. 73-82.

36. Sakakibara, R., et al., Micturitional disturbance in Wernicke's encephalopathy. Neurourology and Urodynamics, 1997. 16(2): p. 111-115.

37. Zempleni, M.Z., et al., Cortical substrate of bladder control in SCI and the effect of peripheral pudendal stimulation. Neuroimage, 2010. 49(4): p. 2983-2994. 


\section{Chapter 9}

Summary 
In this thesis we focused on sensory connections of the lower urinary tract with the periaqueductal gray matter (PAG). In this work we used various anatomical and physiological methods to explore possible connections between the bladder and the PAG.

To find out the current status of the developments in the field, in chapter 2 , we made an overview of the research done on the bladder-PAG connections. This review also takes into account, the state of the art functional imaging techniques respecting the PAG activity. In line with previous studies, defining opposite autonomic functions in the ventrolateral (vlPAG) and dorsolateral PAG columns, we differentiated the function of these two columns respecting the control of the bladder physiology. Finally, we described various central neuropathologies affecting the PAG, with subsequent micturition disorders.

In the next chapter we tried to develop a feasible method for applying peripheral neuromodulation in freely moving rats. This method would then be used in chapter 3 for electrical stimulation of the bladder. We took advantage of the rat's loose skin jacket to guide the wires in a safe location, readily accessible by the experimentalist. This is well tolerated by the animal and simplifies the behavioural tasks.

In chapter 4 we tried to define the exact PAG columns which are activated by bladder stimulation. We exposed the rats to a controlled stimulation paradigm and excluded pain and anxiety by performing behavioural tests. Subsequently, we compared different PAG columns respecting cFos expression. This led to a better understanding of the anatomical destination of ascending sensory signals from the bladder. Yet the exact cell types that were activated could not be differentiated in this way.

Following the previous results, we aimed for phenotyping of the particular neurons which were activated. To accomplish this, we probed five common cell types, namely dopaminergic, serotoninergic, glutamatergic, GABAergic and nNOS synthesizing neurons, by labelling them with the corresponding markers. We then observed which of them overlap with those activated cells expressing cFos. The neurons expressing the stimulatory neurotransmitter glutamate were significantly more activated in the group with electrical stimulation of the bladder, relative to the sham group.

Chapter 6 tests how the firing pattern of the neurons in the PAG would respond to the peripheral electro-stimulation. Firing pattern of the vlPAG was measured by single unit recording, after electrical stimulation of the bladder by the same parameters as described in chapter 4 . We observed an overall decrease in the firing rate during the stimulation period. This reveals instantaneous influence of the incoming signals from a stimulated bladder, on vlPAG neurons. It would be interesting to find out if those responsive neurons were the same glutamatergic neurons found in chapter 5 . However, this may need more sophisticated techniques to be accomplished in future studies.

That some forms of neuropathology are not always restricted to the brain and spinal cord, but have to be seen as a more generalized disease, is supported by the findings described in Chapter 7. In this chapter, we showed that the bladders of transgenic Alzheimer mice 
may malfunction, irrespective of their central connections, based on peripheral locations or sequalae of the disease.

The above findings highlight the crucial role of the PAG on lower urinary tract systemsphysiology. Many central neuropathologies are behind functional bladder disorders. Yet, each individual neuropathology must be thoroughly investigated both centrally and also at the end organ, as this may reveal interesting future avenues for diagnosis and treatment of functional and neuro-urological dysfunctions. 


\section{Samenvatting}

Dit proefschrift richt zich op de verbindingen van gevoelszenuwen vanuit de lage urinewegen naar een specifiek centraal hersengebied, het periaqueductale grijs (PAG). Tijdens het onderzoek, dat in dit proefschrift is beschreven, hebben we verschillende anatomische en fysiologische methoden gebruikt om de mogelijke connecties tussen blaas en PAG te detecteren en te karakteriseren.

In hoofdstuk 2 wordt de huidige stand van de ontwikkelingen op het gebied van verbindingen tussen urineblaas en PAG beschreven. Ook wordt in dit review ingegaan op de huidige stand van zaken met betrekking tot functionele beeldvormingstechnieken voor PAG activiteit. Zoals ook bij eerdere studies werd gevonden hebben 2 specifieke gebieden in het PAG: de ventrolaterale en de dorsolaterale kolom tegenovergestelde autonome functies. Eenzelfde functie differentiatie werd hierin gevonden voor de blaas en lage urineweg fysiologie. Als laatste werd de invloed op het PAG van verschillende neurologische afwijkingen beschreven samen met de hiermee samenhangende blaas disfuncties.

In het volgende hoofdstuk hebben we een bruikbare methode geprobeerd te ontwikkelen om op een perifere locatie neuromodulatie te kunnen toepassen bij vrij bewegende ratten. Deze methode werd gebruikt in hoofdstuk 3 voor elektrische stimulatie van de blaas. We hebben hierbij gebruik gemaakt van de losse huid bij ratten om de draden onderhuids te tunnelen naar een locatie onder het nekvel. Daar is makkelijk toegang te krijgen voor de onderzoeker. Het is verder een plaats waar dit goed door het dier wordt getolereerd en ook onderzoek met gedragstaken mogelijk maakt.

In hoofdstuk 4 hebben we gedefinieerd welke kolommen van het PAG precies worden geactiveerd bij stimulatie van de blaas. Hiervoor hebben we bij ratten een standaard gecontroleerd stimulatieschema aangeboden en daarnaast ook gedragstesten gedaan om een mogelijke activatie van het PAG door angst of pijn uit te sluiten. De activatie werd in kaart gebracht door de expressie van cFos te evalueren in de verschillende PAG kolommen. Dit gaf ons een beter begrip van de anatomische locatie waar signalen van de gevoelszenuwen van de blaas binnenkomen. In dit onderdeel van de studie kon echter niet worden bepaald welke celtypes op deze locaties in het PAG werden geactiveerd.

Hierna werd, op grond van deze uitkomsten, een vervolg studie uitgevoerd met als doel, het karakteriseren van de specifieke zenuwcellen, die door blaasstimulatie werden geactiveerd. De aanwezigheid van vijf vaak voorkomende signaalstoffen werd getest met immunofluorescentie op het zenuwweefsel van het PAG: dopamine, serotonine, glutamaat, GABA en nNOS. Van de zenuwcellen die hierop positief reageerden werd ook in kaart gebracht of ze tegelijkertijd op cFos reageerden na blaasstimulatie. De zenuwcellen die glutamaat bevatten werden significant vaker geactiveerd in de groep die blaas stimulatie kreeg vergeleken met de controlegroep.

Hoofdstuk 6 beschrijft hoe het elektrische excitatie patroon van de zenuwcellen in het ventrolaterale deel van het PAG verandert onder invloed van blaasstimulatie. Met behulp 
van een enkele unit elektrofysiologische registratie uit cellen in deze regio werd de reactie geanalyseerd op blaasstimulatie met dezelfde parameters beschreven in hoofdstuk 4. Gedurende de blaasstimulatie werd een algemene verlaging van de vuurfrequentie gezien in deze regio. Dit betekent dat de signalen uit de blaas een invloed hebben op ook andere inkomende signalen in het PAG. We weten nog niet of dit ook veroorzaakt wordt door de glutamaat bevattende zenuwcellen zijn die in hoofdstuk 5 werden beschreven. Om de exacte aard en connecties van de gestimuleerde cellen te bepalen zijn meer specifieke andere technieken nodig die in de nabije toekomst kunnen worden toegepast.

Meerdere vormen van neurogene ziekte zijn niet alleen beperkt tot de hersenen en het ruggenmerg, maar moeten worden gezien als een meer algemeen in het lichaam verspreide ziekte. Deze hypothese wordt ondersteund door de bevindingen van hoofdstuk 7. In dat hoofdstuk werd aangetoond dat blazen van muizen met een genetische modificatie die codeert voor de ziekte van Alzheimer, zelfs als deze blazen worden getest buiten het lichaam, en dus losgekoppeld van het centrale zenuwstelsel, ook een afwijkende functie laten zien. Hierdoor is er een sterke aanwijzing dat deze ziekte ook in een orgaan als de blaas afwijkingen veroorzaakt.

De bevindingen beschreven in dit proefschrift benadrukken dat het PAG een sleutelrol heeft in het functioneren van de lage urinewegen. Veel neurogene afwijkingen gaan gepaard met blaas- of sluitspierfunctie afwijkingen. Bij nieuwe toekomstige neurourologische projecten zal in het onderzoek en in de kliniek zowel in diagnostische als ook in behandel trajecten, aandacht moeten zijn voor centrale neurogene disfunctie maar ook voor hiernaast optredende disfunctie aan het eindorgaan, de blaas of het afsluitmechanisme van de blaas. 


\section{Valorization}

In this thesis, the role of periaqueductal gray (PAG) matter in mediating the afferent bladder signals using well-established expertise, is examined. This project already needed interdisciplinary knowledge utilization and the results will certainly open new avenues where knowledge is collected, interpreted and utilized in an interdisciplinary framework in the future. From a scientific and clinical point of view, there is a foreseeable possibility of identifying novel mechanisms of brain function that would facilitate the accurate targeting of the micturition control sites in the brain, and management of the symptoms in patients with micturition abnormalities. Specifically, understanding the essence and extent of PAG involvement in local and remote neural elements that regulate bladder sensation and micturition behavior will clarify the main components driving the therapeutic benefits of clinical interventions, and the mechanisms that facilitate and that work at cross-purposes in patients. The knowledge utilization aspects of this thesis can be outlined as follows:

\section{Opportunities for Drug Discovery}

In chapters 4 and 5 we investigated the involvement of various neurotransmitter systems in ventrolateral column of the PAG regarding the reception of bladder sensory signals. These experiments revealed the glutamatergic neurotransmission to be the main cellular mechanism mediating these inputs. Glutamatergic neurotransmission has excitatory influence over its target regions, and has been under extensive pharmacological investigation. Here, we found out a substantial target for therapeutic applications (Table 1).

Note that most of the drugs mentioned in table 1 are active either in epileptic or neurodegenerative disorders. Particularly the latter may affect the PAG as well. Besides this, many vacant drug mechanisms exist with potential for synthesis of novel agents. For example drugs which act over synthesis or storage of the glutamate, or those that may act on reuptake or degradation of the released drug into the synapse. Each potential agent may act by facilitating or inhibiting either phase. On the other hand, receptor level mechanisms may be directed towards investigating specific glutamate receptor subtypes, or designing drugs which have agonist, partial agonist or reverse agonist mechanisms of action. Further investigation will have to focus on finding out the exact location of specific glutamate receptor subtypes in specific PAG columns.

These drugs will certainly be active not only on the urological patient, but on all individuals who suffer from dysfunctions in the spectrum of PAG functions including nociception, behavioural and autonomic systems. As detailed above, the impact of this pathway is abundant both because the PAG has a broad spectrum of functions, and there 
are a lot of drugs active in this system. Thus, any pharmacological intervention in this system in the PAG requires detailed understanding of the associated micro-circuitry and need a more or less systems physiological approach. Additionally, one may investigate specific side effects or potential benefits that each of the already discovered drugs (Table 1) have on urodynamic function. Particularly for clinical investigators, that would be a great opportunity to expand on.

Table 1. So far clinically tested drugs or agents active on the glutamatergic system can be classified as follows (some drugs have multiple mechanisms of action):

- Glutamate release inhibitors :

○ Levetriacetam

- Riluzole

- phenobarbital

- Glutamate receptor antagonists

- NMDA antagonists:

- NO

- Xe

- PCP

- Ketamine

- Alcohol

- acamprosate

- Methadone

- Amantadine

- memantine

- Riluzole

- Dizocilipine [ MK 801 ]

- Felbamate

- AMPA antagonists:

- phenobarbital

- Topiramate

- lamotrigine

- Philanthotoxin [ produced by Wasps ]

\section{Potential targets for surgical intervention}

Furthermore, there is a possibility of modulating PAG function by deep brain stimulation (DBS). In contrast to peripheral neuromodulation, central neuromodulation has not been performed for urological disorders. This approach particularly has the advantage of treating the central etiologies when they exist, for example in extrapyramidal disease with micturition symptoms, rather than symptomatic treatment. Moreover, there are promising future techniques for neuromodulation of micro circuits such as DREADD (designer receptors exclusively activated by designer drugs), or magnetic stimulation using nano-particles, which may ultimately turn central neuromodulation into a non- 
invasive technique. Additionally, new DBS paradigms have the advantage of being on demand, with a patient controlled interactive system. This decreases the adverse autonomic effects, as well as enabling the patient to increase the amount of stimulation in certain circumstances. Defining and programming for different types of stimulation, for example a change of stimulation frequency or application of burst algorithms, would also allow to specifically restore a particular function. The latter is a promising and an only sporadically explored field in urological applications for neurostimulation.

\section{Diagnostic applications}

In chapter 2 we elaborated on some common diseases affecting the PAG and compromising the bladder sensation and micturition. We also showed that many of these pathologies can be detected by modern imaging techniques, especially specific MRI techniques such as fMRI. This ultimately lead us to the conception that, the PAG must be included in future diagnostic and therapeutic patient management algorithms. This would make clinicians aware of the possible involvement of this structure in pathophysiology of disease, and guide them to more appropriate decisions. More precise evaluation of patient condition may be achieved by Magnetic Resonance Spectroscopy (MRS), to find out the chemical composition of a particular PAG segment. This would aid in the diagnosis of many diseases. For example, it may aid in defining the state of the involvement of glutamatergic PAG cells in multiple system atrophy, and correlating it with the severity of symptoms. With the upcoming high resolution MRI machines, we can gain more insight about the specific columns of the PAG.

\section{Novel preclinical techniques}

In addition to the above mentioned clinical derivatives that may result from this thesis, we introduced two techniques to be used by researchers in the associated fields.

In chapter 3, we introduced a method for peripheral neuromodulation in rodents, which would be more comfortable for the animal and for the experimentalist. This method may be used by allied research groups which have an interest in chronic peripheral stimulation, recording or monitoring in live rats, like those working in urological, gastrointestinal and spinal research. This technique in general, engages an access point over the rat skull and redirects the connections from the subcutaneous space.

In chapter 6, we described a slight technical modification for observation of the pontamine sky blue dot. The pontmaine sky blue dye is used for localization of a glass micropipette electrode. The very small injection area adjacent to the tip of the electrode, accompanied by light scattering in bright field microscopy, makes the visualization difficult. On the other hand, we exploited the fluorescence of pontmaine to observe minute injection quantities in the dark field fluorescence microscope. This increases the contrast to a great deal and makes the visualization much easier. To aid the background 
clarification we used Hoechst staining. This technique may be readily used by other electrophysiologists using glass micropipettes as recording electrodes.

Our translational neuromodulation group at MUMC will facilitate direct utilization of the scientific outcomes in clinical practice. This group, comprised of integrated collaboration between the departments of neuroscience, neurology, neurosurgery, anesthesiology, psychiatry, urology, colorectal surgery and ENT, provides a unique team to conduct translational urodynamic/neuromodulation studies. Close collaboration and communication between basic scientists and clinicians in our team will lead to a successful implementation of preclinical findings in clinical practice.

Our novel insights will be shared with patient organizations, health care professionals and scientific societies. From an academic perspective, our results have been/will be published in peer-reviewed international journals and will be presented at (inter)national scientific meetings 


\section{Acknowledgment}

Dear Professor van Koeveringe, in contrast to our initial hesitance and worries, this path turned to the right direction. I appreciate your endurance toward the goal.

Dear Ali, I for sure owe a great deal to your involvement in this project, which was much more than just a copromoter, but like an older brother.

Dear Sandra, I appreciate your efforts to help me bring the electrophysiology experiment to an end, and for your guidance throughout the project.

Dear Celine, I appreciate your primary research on the PAG and the provision of the initial research material for us to expand on.

I appreciate the people in the neuroscience department, especially professor Temel and professor Steinbusch for providing scientific facilities and guidance relevant to our research.

I appreciate Sajjad and Ramona for their organizational or scientific support.

Special thanks to my uncle for providing me his facilities to be able to work here.

Many thanks to LAB technicians in the department of neuroscience for their routine and on-demand help; Helen, Denise, Marjan, Marcella \& Rachelle.

I would also like to thank the secretaries of Urology; Nancy, Anja and Shelly, secretaries of neuroscience \& ME\&NS; Rachelle \& Marie-Therese, as well as staff from MH\&NS, specially Ankie \& Tom, for their support.

I owe a great deal to Maurice Stegermann of MUMC facility services. The grand YAMAHA he provided me, with such a spectacular acoustic resonance, used to make my best moments of existence. 


\section{Curriculum Vitae}

Aryo Zare was born in 1985 in Sary, Iran. Long before his interest in biology, he had interest in music which has increased throught the time. As early as 1995, he had the honor of achiving the $4^{\text {th }}$ rank in Piano performance in the Fajr national music festival in Tehran, in the corresponding age group while he was 10 years old.

In the year 2000 he succeeded in enetering Iran's elite highschools (NODET, SAMPAD). In 2003 he attempted to win Iran's national biology olympiads. He passed the first phase and achieved the $13^{\text {th }}$ national rank of the preparatory exam. Allthough he could not achive being a medalist, his interest in biomedical sciences continued and urged him to enter Mashhad University in 2004, one of the highest ranking medical schools in Iran.

After graduating in 2012, he moved to Kharg island in the Persian Gulf to practice medicine as a general practitioner for 2 years. Though associated with difficulties, the life on an island, together with his adventurous attitude, was some novel experience that non of his collegues back in the medical school could ever have imagined.

He continued pursuing his interest in the biomedical field by enrolling in a $\mathrm{PhD}$ program at Maastricht University in May 2015. Though this as well was not a simple trial, ultimately, he proved again, that he can overcome big challenges. 


\section{Publications:}

- Zare A, Jahanshahi A, Meriaux C, Steinbusch HW, van Koeveringe GA (2018) Glutamatergic cells in the periaqueductal gray matter mediate sensory inputs after bladder stimulation in freely moving rats. Int J Urol. doi:10.1111/iju.13562

- Zare A., et al., The Role of the Periaqueductal Gray Matter in Lower Urinary Tract Function. "Molecular Neurobiology" DOI: 10.1007/s12035-018-1131-8

- Zare A., et al., Electrophysiological charachteristics of ventrolateral periaqueductal gray neurons and their correlation with bladder sensory processing. Conditionally accepted (major revision), "Brain Research Bulletin"

- Celine Meriaux, Ramona Hohnen, Sandra Schipper, Aryo Zare, Ali Jahanshahi, Lori Birder, Yasin Temel, Gommert A. van Koeveringe. Neuronal activation in the periaqueductal gray matter upon electrical stimulation of the bladder. "Frontiers in Cellular neuroscience" DOI: 10.3389/fncel.2018.00133

- Afsharzadeh M, Tayarani-Najaran Z, Zare A, Mousavi SH (2012) Protective Effect of Scutellaria litwinowii Extract on Serum/Glucose-Deprived Cultured PC12 Cells and Determining the Role of Reactive Oxygen Species. J Toxicol 2012:413279. doi:10.1155/2012/413279 
